
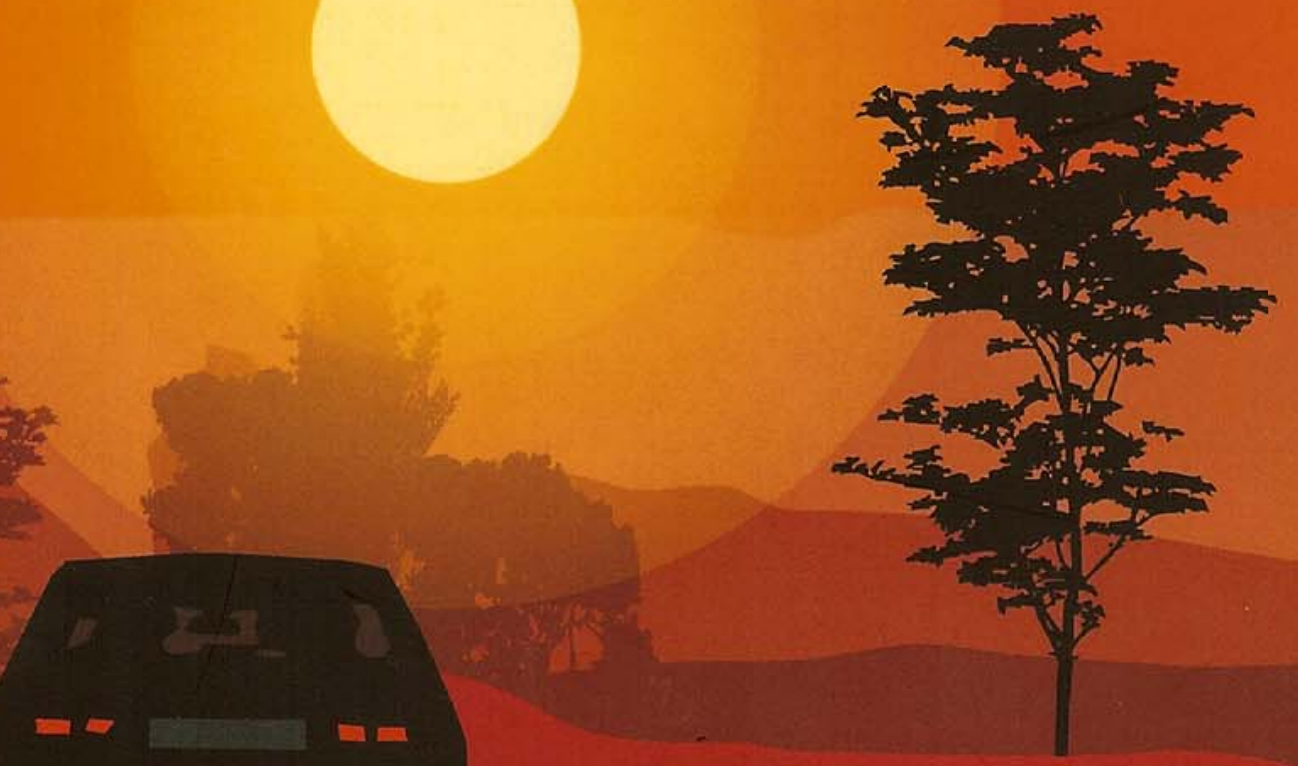

\title{
CONDUCCIÓN Y
}

SUSTANCIAS PSICOACTIVAS

(ALCOHOL, DROGAS Y MEDICAMENTOS):

DE LA MAGNITUD DEL PROBLEMA A LA INTERVENCIÓN EN SEGURIDAD VIAL

Inmaculada Fierro 


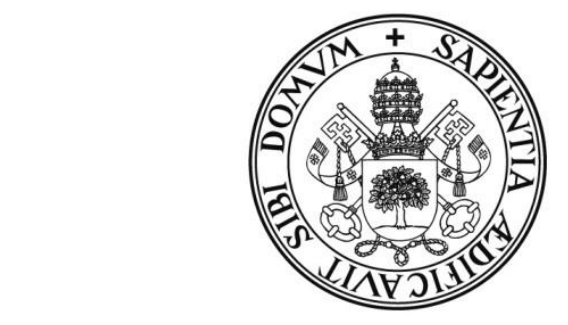

\title{
Universidad deValladolid
}

FACULTAD de MEDICINA

DEPARTAMENTO de BIOLOGÍA CELULAR, HISTOLOGÍA y

FARMACOLOGÍA

TESIS DOCTORAL:

\section{CONDUCCIÓN Y SUSTANCIAS PSICOACTIVAS (ALCOHOL, DROGAS Y MEDICAMENTOS): \\ DE LA MAGNITUD DEL PROBLEMA A LA INTERVENCIÓN EN SEGURIDAD VIAL}

Presentada por M. Inmaculada Fierro Lorenzo para optar al grado de Doctora por la Universidad de Valladolid

\author{
Dirigida por \\ F. Javier Álvarez González
}



A Jesús, Eva y Claudia 

Yo siempre he sido lento, una persona que se angustia y lucha con cada frase, e incluso en mis mejores días no hago más que avanzar centímetro a centímetro, arrastrándome sobre el vientre como un hombre perdido en el desierto. La palabra más corta está rodeada de kilómetros de silencio para mí, y hasta cuando consigo poner esa palabra en la página, me parece que está allí como un espejismo, una partícula de duda que brilla en la arena.

(Leviatán, Paul Auster) 

Agradecimientos

Quiero expresar mi más sincero agradecimiento a todas las personas e instituciones que, en mayor o menor medida, han contribuido a la realización de esta Tesis Doctoral y en especial:

Al Prof: Javier Álvarez, director de esta Tesis Doctoral y que ha confiado en mi trabajo desde que llegué al Departamento de Farmacología. Desde el primer momento me ofreció la oportunidad de colaborar en sus interesantes proyectos y puso a mi disposición los medios a su alcance, tanto para mi formación como para desarrollar mis propias ideas.

A los Profesores Luis Debán y Rafael Pardo que dirigieron mi trabajo en otros momentos y con los que nunca he dejado de aprender. Siempre me apoyaron y animaron para realizar la tesis.

A los coautores de los artículos que forman parte de este compendio $y$ que tan amablemente han permitido su presentación como parte de esta tesis, en especial, a la Dra. Trinidad Gómez-Talegón con la que he compartido tantas horas de trabajo.

A Mr. Alan Hynds por su empeño, semana tras semana, en mejorar nuestro nivel de inglés $y$ el de nuestros artículos.

A Begoña Valdés por su siempre amable colaboración cada día de trabajo.

A todos ellos, muchas gracias

Inma 



\section{ORGANIZACIÓN DE LA TESIS}

De acuerdo con la normativa vigente,

RESOLUCIÓN de 3 de diciembre de 2012, del Rector de la Universidad de Valladolid, por la que se acuerda la publicación de la normativa para la presentación y defensa de la tesis doctoral en la Universidad de Valladolid (publicado en BOCYL 243 de 19 de diciembre), esta Tesis Doctoral se presenta como compendio de publicaciones.

Se incluyen 6 artículos publicados en revistas científicas con factor de impacto y un último artículo aún no publicado (referencias en orden descendente según fecha de publicación):

Fierro, I., Gonzalez-Luque, J.C., Alvarez, F.J., 2014. The relationship between observed signs of impairment and THC concentration in oral fluid. Drug Alcohol Depend. 144, 231-238.

Fierro, I., Gomez-Talegon, T., Alvarez, F.J., 2013. The Spanish pictogram on medicines and driving: The population's comprehension of and attitudes towards its use on medication packaging. Accid Anal Prev. 50, 1056-1061.

Benavidez, D.C., Flores, A.M., Fierro, I., Alvarez, F.J., 2013. Road rage among drug dependent patients. Accid Anal Prev. 50, 848-853.

Gomez-Talegon, T., Fierro, I., Gonzalez-Luque, J.C., Colas, M., Lopez-Rivadulla, M., Alvarez, F.J., 2012. Prevalence of psychoactive substances, alcohol, illicit drugs, and medicines, in Spanish drivers: a roadside study. Forensic Sci Int. 223, 106-113.

Fierro, I., Morales, C., Alvarez, F.J., 2011. Alcohol use, illicit drug use, and road rage. J Stud Alcohol Drugs. 72, 185-193.

Fierro, I., Gomez-Talegon, T., Alvarez, F.J., 2010. Road-rage in the general population. Gac Sanit. 24, 423-427.

Driving under the influence of drugs in Spain, 2013: decrease prevalence after introduction of onsite drug test (sin publicar). 



\section{CONDUCCIÓN Y SUSTANCIAS PSICOACTIVAS}

(ALCOHOL, DROGAS Y MEDICAMENTOS): DE LA MAGNITUD DEL PROBLEMA A LA INTERVENCIÓN EN SEGURIDAD VIAL

Inmaculada Fierro 2015 

Capítulo 1:Introducción general 15

$\begin{array}{ll}\text { Abreviaturas y acrónimos } & 17\end{array}$

1. Antecedentes 19

1.1. Las drogas y la conducción de vehículos 22

1.2. Medicamentos y conducción 25

1.3. Signos de deterioro en conductores y presencia de 27 sustancias

1.4. Agresividad vial $\quad 30$

2. Objetivos 33

3. Metodología y resultados por objetivos 35

3.1. Prevalencia y evolución del consumo de sustancias 35 psicoactivas por los conductores españoles

3.1.1.Metodología $\quad 35$

3.1.2. Resultados 41

3.2. El pictograma español sobre medicamentos y conducción 49

3.2.1.Metodología $\quad 49$

3.2.2. Resultados $\quad 52$

3.3. Relación entre los signos de deterioro en conductores y las $\quad 55$ concentraciones de THC en su fluido oral

3.3.1.Metodología $\quad 55$

3.3.2. Resultados 58

3.4. Agresividad vial $\quad 63$

3.4.1.Metodología 63

3.4.2. Resultados 68

4. Conclusiones por objetivos 75

5. Referencias 79

Capítulo 2: Prevalence of psychoactive substances, alcohol, illicit 85 drugs, and medicines, in Spanish drivers: a roadside study

$\begin{array}{ll}\text { Abstract } & 87\end{array}$

1. Introduction 89

2. Material and methods 91

3. Results 96

4. Discussion 105

5. References 111

Capítulo 3: The Spanish pictogram on medicines and driving: The 115 population's comprehension of and attitudes towards its use on medication packaging

Abstract 
1. Introduction

2. Material and methods

3. Results

4. Discussion

5. References

Capítulo 4: The relationship between observed signs of impairment and THC concentration in oral fluid

Abstract

1. Introduction

2. Material and methods

145

3. Results

149

4. Discussion

156

5. References

161

APPENDIX A. Supplementary data

165

Capítulo 5: Agresividad vial en la población general

169

Resumen

171

Abstract

172

1. Introducción

173

2. Métodos

174

3. Resultados

178

4. Discusión

183

5. Referencias

186

Capítulo 6: Alcohol use, illicit drug use, and road rage

189

Abstract

191

1. Introduction

193

2. Methods

195

3. Results

202

4. Discussion

206

5. References

210

215

Capítulo 7: Road rage among drug dependent patients

Abstract

1. Introduction

$\begin{array}{ll}\text { 2. Material and methods } & 220 \\ \text { 3. Results } & 224\end{array}$

4. Discussion 231

5. References 235

Capítulo 8: Driving under the influence of drugs in Spain, 2013: 239 decrease prevalence after introduction of onsite drug test

Abstract

1. Introduction

2. Methods

3. Results

4. Discussion

5. References 
Capítulo 1

Introducción General 



\section{Abreviaturas y acrónimos}

AUC Área bajo la curva (del inglés, Area Under the Curve)

BACs Concentración de alcohol en sangre (del inglés Blood Alcohol Concentrations)

CAGE Test para la detección del consumo problemático de alcohol

DAS Driving Anger Scale

DAX Driving Anger Expession Inventory

DE Desviación Estándar

DGT Dirección General de Tráfico

DRUID DRiving Under the Influence of Drugs, Alcohol and Medicines (Proyecto europeo)

E Especificidad

FN Falsos Negativos

FP Falsos Positivos

g/l Gramos por litro

IC Intervalo de Confianza

IMMORTAL Impaired Motorists, Methods of Roadside Testing and Assessment for Licensing (Proyecto de investigación)

MDA 3,4-metilendioxanfetamina

MDEA 3,4-metilendioxietilanfetamina

MDMA 3,4-metilendioximetanfetamina

$\mathrm{mg} / \mathrm{l} \quad$ Miligramos por litro

$\mathrm{ng} / \mathrm{ml} \quad$ Nanogramos por mililitro

NHTSA National Highway Traffic Safety Administration

OMS Organización Mundial de la Salud

PADS Propensity for Driving Anger Scale

OR Odds Ratio

RD Real Decreto

ROC Característica operativa del receptor (del inglés, Receiver Operating Characteristic)

S Sensibilidad

SNC Sistema Nervioso Central

THC $\quad \Delta^{9}$-Tetrahidrocanabinol 
VG Valor Global de la prueba diagnóstica

VN Verdaderos Negativos

VP Verdaderos Positivos

VPN Valor Predictivo Negativo

VPP Valor Predictivo Positivo 


\section{Antecedentes}

Los accidentes de tráfico han sido motivo de preocupación en nuestra sociedad, prácticamente desde que el automóvil se inventó en 1886. Desde finales del siglo XIX, más de treinta millones de personas han muerto en el mundo, como consecuencia de las lesiones ocasionadas por colisiones de tráfico y la OMS prevé que los traumatismos por accidentes de tránsito aumentarán para pasar a ser la quinta causa principal de mortalidad en 2030 (Figura 1). Los accidentes de tráfico, o más correctamente, las lesiones derivadas de las colisiones de tráfico constituyen, por lo tanto, un grave problema de salud pública a nivel mundial $[1,2]$.

\begin{tabular}{|c|c|c|c|c|c|}
\hline \multicolumn{3}{|c|}{ TOTAL 2004} & \multicolumn{3}{|c|}{ TOTAL 2030} \\
\hline NO. DE ORDEN & PRINCIPALES CAUSAS & $\%$ & NO. DE ORDEN & PRINCIPALES CAUSAS & $\%$ \\
\hline 1 & Enfermedad isquémica del corazón & 12,2 & 1 & Enfermedad isquémica del corazón & 14,2 \\
\hline 2 & Enfermedad cerebrovascular & 9,7 & 2 & Enfermedad cerebrovascular & 12,1 \\
\hline 3 & Infecciones de las vías respiratorias inferiores & 7,0 & 3 & Enfermedad pulmonar obstructiva crónica & 8,6 \\
\hline 4 & Enfermedad pulmonar obstructiva crónica & 5,1 & 4 & Infecciones de las vías respiratorias inferiores & 3,8 \\
\hline 5 & Enfermedades diarreicas & 3,6 & 5 & Traumatismos por accidentes de tránsito & 3,6 \\
\hline 6 & VIH/sida & 3,5 & 6 & Cánceres de la tráquea, los bronquios y el pulmón & 3,4 \\
\hline 7 & Tuberculosis & 2,5 & 7 & Diabetes mellitus & 3,3 \\
\hline 8 & Cánceres de la tráquea, los bronquios y el pulmón & 2,3 & 8 & Enfermedad cardíaca hipertensiva & 2,1 \\
\hline 9 & Traumatismos por accidentes de tránsito & 2,2 & 9 & Cáncer del estómago & 1,9 \\
\hline 10 & Prematuridad y bajo peso al nacer & 2,0 & 10 & $\mathrm{VIH} /$ sida & 1,8 \\
\hline 11 & Infecciones neonatales y otras ${ }^{a}$ & 1,9 & 11 & Nefritis y nefrosis & 1,6 \\
\hline 12 & Diabetes mellitus & 1,9 & 12 & Lesiones autoinfligidas & 1,5 \\
\hline 13 & Paludismo & 1,7 & 13 & Cáncer del hígado & 1,4 \\
\hline 14 & Enfermedad cardíaca hipertensiva & 1,7 & 14 & Cáncer colorectal & 1,4 \\
\hline 15 & $\begin{array}{l}\text { Asfixia del nacimiento y traumatismo del } \\
\text { nacimiento }\end{array}$ & 1,5 & 15 & Cáncer del esófago & 1,3 \\
\hline 16 & Lesiones autoinfligidas & 1,4 & 16 & Violencia & 1,2 \\
\hline 17 & Cáncer del estómago & 1,4 & 17 & Alzheimer y otras demencias & 1,2 \\
\hline 18 & Cirrosis del hígado & 1,3 & 18 & Cirrosis del hígado & 1,2 \\
\hline 19 & Nefritis y nefrosis & 1,3 & 19 & Cáncer de mama & 1,1 \\
\hline 20 & Cáncer colorectal & 1,1 & 20 & Tuberculosis & 1,0 \\
\hline
\end{tabular}

Figura 1 Principales causas de mortalidad en 2004 y estimación de la OMS para 2030. Tomado del Informe sobre la Situación Mundial de la Seguridad Vial. OMS (2009) [1].

Los primeros accidentes de tráfico fueron considerados, al igual que otro tipo de accidentes, hechos fortuitos en los que el azar constituía el factor determinante. Sin embargo, a mediados del siglo XX la concepción del accidente cambió sustancialmente. Las numerosas investigaciones llevadas a cabo en los 
accidentes han permitido identificar los diferentes factores que contribuyen a que éstos sucedan $y$, en general, se puede concluir que con la eliminación de uno o más de dichos factores, el accidente podría haberse evitado (Figura 2).

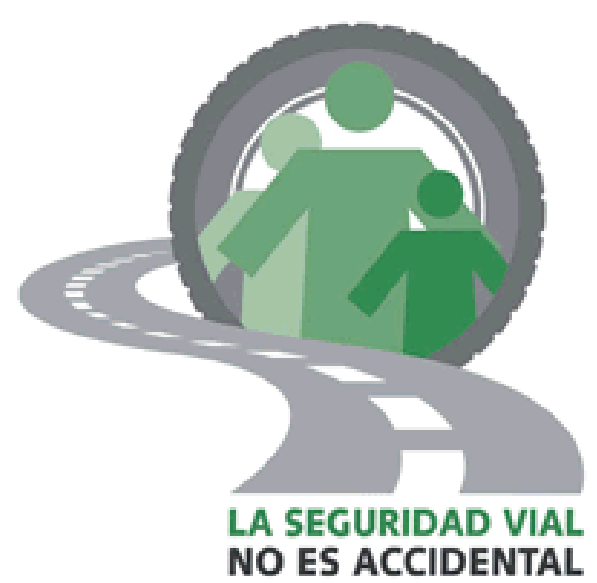

Según el director de la OMS: "Demasiado a menudo, la seguridad vial se considera un asunto de política de transporte, no un problema de salud pública, y los traumatismos causados por el tránsito se califican sólo de «accidentes», aunque la mayoría de ellos se podrían prevenir".

Desde el punto de vista de la salud pública sería más correcto utilizar los términos "colisiones de tráfico" o "lesiones por colisión de tráfico" en vez de "accidentes de tráfico".

Figura 2 La seguridad vial no es accidental. Eslogan del Día Mundial de la Salud en el año 2004 [3].

La identificación de los factores de riesgo que intervienen para que ocurra un accidente de tráfico es motivo de numerosas investigaciones, y aunque el accidente suele ser el resultado de la confluencia desfavorable de múltiples factores (el vehículo, la carretera, la señalización, la vigilancia, etc.), algunas investigaciones aseguran que el "factor humano" es determinante en el 70 a $90 \%$ de los siniestros en la carretera.

Cuando nos referimos a los accidentes de tráfico, el factor humano está constituido por todas aquellas circunstancias que dependen únicamente del conductor, tanto si son debidas a su aptitud para conducir (educación vial, pericia en la conducción, limitaciones físicas, etc.), como a su actitud frente a la conducción (conducir cansado, usar el teléfono móvil, conducir bajo el efecto de sustancias, etc.).

Con la investigación realizada para el presente trabajo se analizan diferentes aspectos del "factor humano" relacionados con la conducción, como son el consumo de sustancias por parte de los conductores (alcohol, drogas ilegales y medicamentos) [4-6] y la agresividad vial (Road rage) [7-9]. Respecto al consumo, se ha analizado la prevalencia de sustancias en conductores [4] y 
además, en el caso del alcohol y el THC, se ha analizado su relación con la prevalencia de signos de deterioro, dependiendo de la concentración de estas sustancias en el organismo [5], Por otra parte, se contemplan dos intervenciones llevadas a cabo en materia de seguridad vial: la introducción del pictograma sobre medicamentos y conducción en el envase de algunos medicamentos [10], y la realización de controles aleatorios de carretera, en los que recientemente se ha introducido el fluido oral como matriz para determinar el consumo de drogas por parte de los conductores [4-6].

Las intervenciones llevadas a cabo, cuyo fin último es reducir la accidentabilidad por tráfico, es de esperar que tengan un efecto modificador del factor humano. Es por esto, que en el último capítulo de este compendio de publicaciones (Capítulo 8), se ha analizado el cambio en la prevalencia de sustancias en conductores, cinco años después de haber realizado el primer análisis $[4,6]$.

Respecto a la introdución del pictograma sobre medicamentos y conducción (Capítulo 3), se ha analizado la probabilidad de un cambio de actitud en el conductor (cambio en la frecuencia de conducción) si tuviera que conducir y tomara un medicamento con el pictograma impreso en su envase [10].

Aunque la agresividad vial puede depender de múltiples factores, su análisis en el contexto de este trabajo se justifica por el hecho de que, en varios estudios previos, se ha observado que tanto las víctimas como los agresores implicados en situaciones de agresividad vial, tienen un riesgo significativamente mayor de verse involucrados en accidentes de tráfico. En las tres publicaciones sobre agresividad vial (road rage) incluidas [7-9], se analizan factores sociodemógráficos (Capítulo 5)[7], así como la posible relación entre el consumo y actitudes relacionadas con el consumo de sustancias por parte de los conductores y la agresividad vial (Capítulos 6 y 7) [8-9]. 


\subsection{Las drogas y la conducción de vehículos}

Todas las drogas afectan a la capacidad para conducir debido a sus efectos sobre el Sistema Nervioso Central (SNC). La forma en que afectan al SNC, y por lo tanto a la conducción, depende del tipo de sustancia: depresora, estimulante, modificadoras de las percepciones [11]. Así, por ejemplo, mientras quienes han ingerido sustancias como la cocaína suelen practicar una conducción agresiva y temeraria, en el caso de la marihuana, las tareas relacionadas con la conducción se realizan de forma lenta y torpe [12-14]. La consecuencia final de la conducción bajo el efecto de sustancias es un aumento de los accidentes de tráfico [15-20], con las consecuencias sociales y personales que de ellos se derivan.

Conducir bajo la influencia de cualquier droga es un motivo de preocupación en el área de la salud pública, ya que no sólo pone en riesgo al conductor, sino también a todos los ocupantes del vehículo y al resto de los usuarios de las vías públicas.

El análisis de la prevalencia de sustancias en los conductores españoles es una cuestión relevante de cara a conocer la magnitud del problema al que nos enfrentamos. Con ello se obtiene una perspectiva de gran utilidad, tanto para planificar y dimensionar las posibles intervenciones de seguridad vial en este campo, como para desarrollar el marco legal en el que estas intervenciones deben realizarse. Por este motivo, junto con la Universidad de Valladolid, la Dirección General de Tráfico (DGT) ha sido un importante colaborador y promotor de esta investigación.

Los resultados recogidos en el capítulo 2 de este compendio [4] ofrecieron, por primera vez en nuestro país, una exaustiva información de la prevalencia de conductores que conducen con presencia de sustancias psicoactivas en su organismo. La investigación realizada ha sido llevada a cabo en el contexto del proyecto europeo DRUID (Driving Under the Influence of Drugs, Alcohol and Medicines http://druid-project.eu/), cuyo objetivo general fue profundizar en el conocimiento del problema del consumo de alcohol, otras drogas y 
medicamentos en los conductores, revisar las posibilidades de intervención y armonizar las actuaciones en el marco de la Unión Europea [21].

Hasta hace pocos años, la detección de otras sustancias diferentes del alcohol en los controles de tráfico suponía realizar una intervención de gran complejidad, debido a la necesidad de disponer de una muestra de sangre u orina. La necesidad de disponer de un método rápido, sencillo y con la posibilidad realizar los análisis "in situ", ha favorecido el que durante la última década se hayan incrementado las investigaciones dirigidas a utilizar el fluido oral como matriz alternativa para la detección de drogas de abuso [22]. Las principales ventajas del fluido oral frente a otras matrices son, que el proceso de toma de toma de muestra es no invasivo (como ocurre en el caso de la sangre) y que se evita el inconveniente de las muestras de orina, que deben ser tomadas bajo la observación de un agente. Por estos motivos, la toma de muestra del fluido oral es mucho mejor aceptada por la gente en general y por los conductores en particular. Además, la presencia de drogas en el fluido oral se correlaciona mejor con el deterioro que la presencia de los metabolitos de drogas en orina [22-25]. La posibilidad de utilizar el fluido oral como matriz para detectar y cuantificar el consumo de sustancias ha sido un factor determinante a la hora de poder generalizar los controles de sustancias en carretera.

En España, los aspectos legales relacionados con la conducción de vehículos están recogidos en el el texto articulado de la Ley sobre Tráfico, Circulación de Vehículos a Motor y Seguridad Vial, aprobado por el Real Decreto Legislativo 339/1990, de 2 de marzo [26]. Aunque las primeras versiones de esta ley ya hacían mención a la prohibición del consumo de drogas en la conducción, ha sido necesario esperar a que los controles para la detección de la presencia de estas sustancias se generalizaran, para poder abordar este grave problema en la seguridad vial.

En diciembre de 2007, con la ley orgánica 5/2010 que modificaba la ley 10/1995 del código penal, se introdujo la obligatoriedad de someterse a las pruebas de detección de drogas en saliva en los controles aleatorios, igual que ya se venía haciendo con la prueba de alcoholemia [27]. Con este cambio en la legislación 
fue posible abordar un proyecto para conseguir una estimación fiable de la prevalencia de sustancias en los conductores españoles [4].

El 20 de marzo de 2014, el Congreso de los Diputados aprobó una importante modificación del texto articulado de la Ley sobre Tráfico, Circulación de Vehículos a Motor y Seguridad Vial en materia de drogas y conducción [28]. En esta reforma que entró en vigor el 9 de mayo de 2014, se recoge por primera vez en una ley española, el principio de tolerancia cero en materia de drogas y conducción. También por primera vez, el nuevo texto recoge de forma explícita que la prueba de detección de drogas en saliva, mediante los dispositivos autorizados, sea el medio preferente para detectar, in situ, la presencia de drogas en el organismo. Además, se especifica que la saliva es la matriz preferente para un posterior análisis de confirmación.

Desde 1990, la ley prohibe conducir "con presencia de drogas en el organismo del conductor, quedando excluidas las sustancias que se utilicen bajo prescripción facultativa y con una finalidad terapéutica, siempre que se esté en condiciones de utilizar el vehículo conforme a la obligación de diligencia, precaución y no distracción establecidad en el artículo 9". Con la nueva ley, conducir con presencia de drogas en el organismo es una infracción catalogada como muy grave; está castigada con una sanción administrativa de 1000 Euros y la retirada de 6 puntos en el carné de conducir [28].

En el caso del alcohol, con la nueva ley las tasas máximas legalmente permitidas para conducir siguen siendo las mismas (tabla 1), pero sí se aumentan algunas de las sanciones previstas en la vía administrativa (Tabla 2). Se incrementan las sanciones, de 500 a 1000 Euros, para los conductores reincidentes, es decir, para aquellos que hubieran sido sancionados en el año inmediato anterior a la alcoholemia positiva. Esta misma sanción de 1000 Euros se aplicará también a aquellos conductores que circulen con una tasa de alcohol que supere el doble de la permitida.

En esta reforma de la Ley de la Seguridad Vial, no se introdujo ninguna modificación en el código penal [29]. 
Tabla 1 Tasas máximas de alcohol permitidas para conducir

\begin{tabular}{lll}
\hline Conductores & Límite en sangre & Límite en aire espirado \\
\hline En general & $0.5 \mathrm{~g} / \mathrm{l}$ & $0.25 \mathrm{mg} / \mathrm{l}$ \\
Profesionales & $0.3 \mathrm{~g} / \mathrm{l}$ & $0.15 \mathrm{mg} / \mathrm{l}$ \\
Noveles & $0.3 \mathrm{~g} / \mathrm{l}$ & $0.15 \mathrm{mg} / \mathrm{l}$ \\
\hline
\end{tabular}

Tabla 2 Sanciones por conducir con presencia de alcohol o drogas

\begin{tabular}{lll}
\hline & Tasa & Sanción \\
\hline \multirow{2}{*}{ Alcohol } & De $0.25 \mathrm{mg} / \mathrm{l} \mathrm{a} 0.50 \mathrm{mg} / \mathrm{l}$ & 500 Euros y 4 puntos \\
& Más de $0.5 \mathrm{mg} / \mathrm{l}$ y reincidentes & 1000 Euros y 6 puntos \\
\hline Drogas & Presencia & 1000 Euros y 6 puntos \\
\hline
\end{tabular}

\subsection{Medicamentos y conducción}

La relación entre el uso de medicamentos y el riesgo de accidentalidad por tráfico ha sido analizada en diversos estudios [30-32]. Además, desde hace algunos años se han realizado varios intentos de clasificar los medicamentos según el grado de deterioro que éstos pudieran producir sobre la capacidad de conducir $[33,34]$. Con el fin de advertir a los usuarios de medicamentos acerca de este posible riesgo, algunos países, como España y Francia, han optado por imprimir un pictograma en los envases de aquellos medicamentos que pudieran suponer un riesgo para la conducción [35-36].

En la Unión Europea es obligatorio para las compañías farmacéuticas, aportar datos sobre los efectos de cada medicamento sobre la capacidad de conducir y manejar maquinaria peligrosa previamente a su comercialización [37]. El prospecto de los medicamentos contiene información advirtiendo de los posibles efectos, ya que existen numerosas evidencias de que algunas de las reacciones adversas de los medicamentos (somnolencia, mareos, visión borrosa) pueden reducir de forma considerable la capacidad de conducción, incrementando el riesgo de sufrir un accidente $[31,32]$. Sin embargo, no todos los usuarios de 
medicamentos leen el prospecto $[38,39]$ y, por otra parte, la legibilidad de éste no siempre está bien valorada por los usuarios [40].

En España, el procedimiento de autorización, registro y condiciones de dispensación de los medicamentos de uso humano fabricados industrialmente, está regulado por el RD 1345/2007 [35]. En este Real Decreto se establece que los medicamentos de nueva autorización que puedan reducir la capacidad para conducir o manejar maquinaria peligrosa, deberán incorporar en el envase un símbolo de advertencia (pictograma).

Desde el año 2011, todos los medicamentos con posibles efectos sobre la capacidad de conducir comercializados en España incorporan el pictograma en su envase (Figura 3). Dicho símbolo debe reunir las siguientes características:

i) Sobre fondo blanco, un triángulo equilátero rojo con el vértice hacia arriba y un coche negro en el interior, muy similar a una señal de tráfico, con la leyenda Conducción: ver prospecto.

ii) El tamaño del pictograma se adaptará al del envase y, en todo caso, el lado del triángulo no será inferior a diez milímetros.

El objetivo del símbolo es el de llamar la atención del usuario, para que lea la información correspondiente a los efectos sobre la conducción y manejo de maquinaria que se encuentra detallada en el prospecto del medicamento [41].

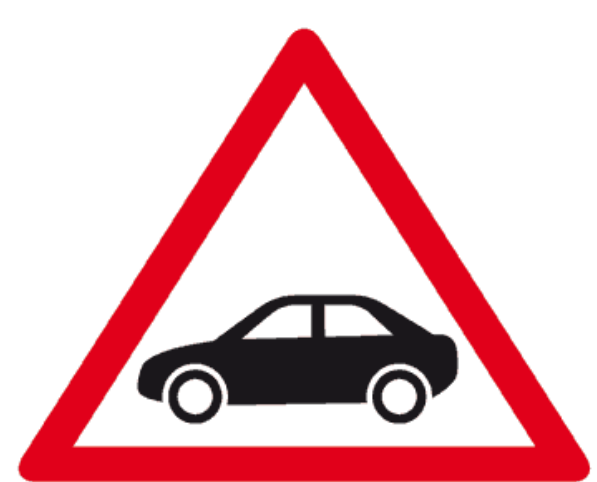

Conducción: ver prospecto

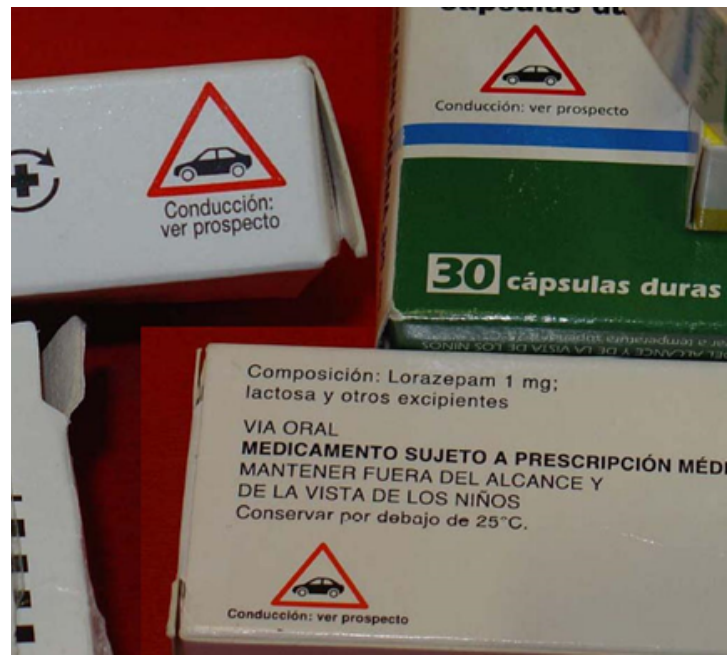

Figura 3 Pictograma español sobre medicamentos y conducción 
La publicación que constituye el capítulo 3 de este compendio, en la que se analiza la comprensión del pictograma español por parte de la población general [10], forma parte de los resultados de un amplio proyecto. En dicho proyecto se desarrollaron distintas actividades con el objetivo de informar y divulgar la relevancia de la implementación del pictograma en los envases de los medicamentos en España, así como, la relación entre medicación y conducción de vehículos y su importancia para distintas poblaciones diana: Médicos, farmacéuticos, pacientes y población en general.

\subsection{Signos de deterioro y conducción con presencia de sustancias}

Respecto al consumo de sustancias que pueden afectar a la capacidad de conducir, las legislaciones vigentes en los diferentes países utilizan, fundamentalmente, tres enfoques para determinar si un conductor involucrado en un accidente o detenido en un control rutinario, debe ser sancionado o tiene alguna responsabilidad penal. El primero de ellos es el enfoque tradicional, el deterioro basado en el efecto de la sustancia. En el segundo, el enfoque "per se", hay dos diferentes versiones, bien con tolerancia cero, o bien con el establecimiento de unos límites de concentración de la sustancia y/o sus metabolitos en la sangre o en otro fluido corporal, basados en evidencias científicas. El tercer enfoque es una combinación de los anteriores, es ilegal conducir con sustancias en concentración superior a un límite establecido y si además se observan signos de deterioro se agrava la pena.

En los países en los que la ley se basa en la observación de un deterioro atribuible al consumo de sustancias, para que un conductor sea penalizado es preciso que éste muestre unos signos claros de afectación [42] y, además, una prueba analítica que confirme la presencia de la sustancia.

En los países que tienen una ley "per se", la superación del límite de concentración establecido es ilegal [43]. Por lo tanto, la evidencia de que la concentración de una sustancia en el cuerpo del conductor excede este límite, supone una prueba legal. En otras palabras, no hay necesidad de investigar si el conductor presenta signos de estar afectado o no. 
En otros países es ilegal conducir con la presencia de alcohol o drogas en el organismo aunque no se observen signos de deterioro, pero, si además se observan signos, la penalización puede ser mayor.

En principio, podría pensarse que el enfoque basado en la presencia de deterioro es el más aconsejable, ya que se penalizaría solo a aquellos que realmente tienen disminuida su capacidad para conducir. Sin embargo, la falta de métodos estandarizados para medir y juzgar el deterioro asociado al consumo de una determinada sustancia hace la evaluación un tanto arbitraria; las disputas legales son comunes y la aplicación de estas leyes supone un elevado coste [44].

En 1998, la National Highway Traffic Safey Administration (NHTSA), publicó en su página web, un informe final elaborado por Sturster y Burns a partir de los trabajos originales de Burns y Moskowitz y de Tharp y cols.[45,46]. Dicho informe titulado, "Validation of the Standardized Field Sobriety Test Battery at BACs Below 0.10\%" ha sido utilizado como un estándar de los tests de sobriedad de campo por los departamentos de seguridad en todo EEUU [47].

Aunque los tests de sobriedad de campo fueron originalmente concebidos para para detectar intoxicación etílica, en muchos países este tipo de tests se han utilizado para detectar el deterioro asociado al consumo de otras sustancia en los conductores. En el caso concreto del cannabis, no existe un total acuerdo acerca de cuáles son los signos observables que revelan un deterioro asociado, específicamente, a su consumo así como de la magnitud de esta correlación.

Por otra parte, mientras algunos autores contemplan la posibilidad de establecer límites legales para la conducción bajo el efecto de marihuana y otras drogas, [44] en la mayor parte de los países de Europa Occidental el estándar es la "tolerancia cero". Ambas consideraciones pueden conllevar distintos problemas tanto técnicos como legales. Dupont y cols. [48] analizaron varios de estos aspectos e hicieron una recomendación sobre el número de pasos que deben ser tenidos en cuenta, en la aplicación de los procedimientos para evaluar la conducción bajo el efecto de las drogas, además, proponían avanzar hacia adelante en la promulgación de leyes sobre drogas "per se". El argumento más 
convincente para la adopción de leyes "per se" fue, previamente a su establecimiento, el que un conductor cuya capacidad para conducir se encontrara deteriorada por el efecto de las drogas (sustancias ilegales) tendría una probabilidad mucho más baja de ser procesado que otro conductor cuyo deterioro fuera debido al consumo de alcohol (sustancia legal). Este dilema se planteó en varios estados de EEUU, porque a pesar de existir ya una ley "per se" para el alcohol, no existía forma práctica o legal de establecer una correlación entre el deterioro y el nivel (concentración) para las sustancias controladas.

En la actualidad, en algunos países con ley "per se", el límite de concentración se ha fijado en el límite de detección del equipo de análisis, lo que supone, de hecho, una ley de tolerancia cero. Según Grotenhermen y cols. [44], "Esto evita la necesidad de una correlación con base científica fiable entre la concentración del fármaco y el nivel de deterioro asociado facilitando el procedimiento. Sin embargo, la tolerancia cero penaliza la presencia en los fluidos corporales, de una droga o sus metabolitos, que no necesariamente se corresponden con el deterioro real".

En España, con anterioridad a la Ley 6/2014, de 7 de abril [28] existía una confusión entre el objeto de la regulación penal y el de la administrativa en lo relativo a las drogas, que ahora en el nuevo texto se precisa.

La Ley prohíbe conducir con presencia de drogas en el organismo del conductor, quedando excluidas las sustancias que se utilicen bajo prescripción facultativa y con una finalidad terapéutica. Esta infracción administrativa se castigará con una sanción de 1000 euros y detracción de 6 puntos.

En cambio, si se conduce bajo la influencia de drogas, la vía ya no es administrativa sino la penal, tal como ya está recogido en el artículo 379.2 del Código Penal [29].

La conducción bajo el efecto de las drogas implicaría, por lo tanto, no solamente presencia de drogas en el organismo sino, además, la presencia de signos de deterioro atribuibles a la sustancia presente en el dicho organismo. 
En relación con este tema, en el capítulo 4 de esta tesis [5] se analiza, para una muestra aleatoria de 2632 conductores españoles, la frecuencia en la que son observados 31 signos de deterioro y su correlación con la presencia de THC en el fluido oral, en diferentes concentraciones.

\subsection{Agresividad vial}

La agresividad vial (en inglés "road rage") es un tema de creciente interés en el ámbito internacional, ya que se ha observado que tanto víctimas como agresores tienen un riesgo significativamente mayor de involucrarse en accidentes de tráfico [49]. En España, la información disponible sobre agresividad vial es muy limitada. Además, no existe una definición unívoca para la expresión "agresividad vial", pero su equivalente en inglés se ha utilizado con frecuencia para describir "un incidente en el cual un conductor o pasajero intenta intimidar, herir e incluso matar a otro conductor, pasajero o peatón, o dañar el vehículo de otra persona" [50-53].

Por otra parte, el consumo de alcohol, cocaína y cannabis se asocia a comportamientos agresivos, a ser víctima de lesiones de diversas causas, y a accidentes de tráfico, pero existen pocos estudios acerca de cómo afecta el consumo de estas sustancias la experiencia de agresividad vial.

La relación entre la agresividad vial y la adopción de determinadas conductas peligrosas en la conducción (por ejemplo, conducir a gran velocidad) explicaría el aumento en el riesgo de sufrir un accidente en el caso de los agresores; en el de las víctimas, la accidentalidad sería consecuencia de otro tipo de comportamiento que podría despertar la agresividad de otros conductores (como la no señalización de una maniobra, o la tendencia a circular muy lentamente en determinadas condiciones de tráfico) [49]. Por otra parte, la agresividad vial suele manifestarse como un proceso interactivo entre víctima y agresor [49], con intercambio de expresiones de hostilidad, lo que en ocasiones puede dificultar la distinción entre víctima y agresor, además de poder involucrar tanto al conductor del vehículo como a los pasajeros, e incluso a los peatones. 
Existen varios cuestionarios destinados a evaluar la agresividad vial. Los más frecuentemente utilizados son: Propensity for Angry Driving Scale (PADS) [54], que identifica a conductores con una mayor probabilidad de verse implicados en situaciones de agresividad vial; Driving Anger Scale (DAS) [55] mide la propensión a experimentar enfado mientras se conduce; Driving Anger Expression Inventory (DAX) [56], mide la forma en de expresar la rabia mientras se conduce; y finalmente, la clasificación según diferentes tipos de comportamiento relacionados con la agresividad vial desarrollada por Smart y cols. $(53,57)$ que cuantifica la frecuencia de verse involucrado en situaciones de agresividad vial, como víctima y/o como agresor, en los 12 meses previos a la realización de la entrevista. Es este último cuestionario, el que ha sido utilizado en las tres publicaciones sobre agresividad vial (road rage) incluidas en este compendio [7-9]. En ellas se analizan factores sociodemógráficos (Capítulo 5), la posible relación entre actitudes relacionadas con el consumo de sustancias por parte de los conductores y la agresividad vial (Capítulo 6) y se compara la agresividad vial entre la población general de Castilla y León y una muestra de drogodependientes que acuden a un centro de tratamiento (Capítulo 7). 



\section{Objetivos}

- Conocer la prevalencia de conductores españoles que conducen con la presencia de sustancias psicoactivas (alcohol, drogas y ciertos medicamentos) y su evolución en el tiempo.

- Evaluar la comprensibilidad del pictograma español sobre medicamentos y conducción impreso en el envase de algunos medicamentos, y su aceptación por parte de los usuarios de medicamentos.

- Analizar la relación entre signos de deterioro en conductores y las concentraciones de THC en el fluido oral y alcohol en aire espirado.

- Analizar la relación entre la agresividad vial y la adopción de ciertas conductas de riesgo relacionadas con el consumo de sustancias y la conducción, en la población general y en pacientes con trastorno por consumo de sustancias.

Dado que cada uno de los objetivos propuestos precisa un desarrollo metodológico bien diferenciado, a continuación se expondrán la metodología y los resultados, estructurados separadamente para cada uno de estos cuatro objetivos. 



\section{Metodología y resultados por objetivos}

\subsection{Prevalencia y evolución del consumo de sustancias psicoactivas por los conductores españoles}

\subsubsection{Metodología}

La población diana está constituida por los conductores de vehículos ligeros, de motor, que circulan por vías públicas españoles (indistintamente de su nacionalidad), tanto en un ámbito urbano como interurbano. Se excluyen, por lo tanto, bicicletas y vehículos de más de 3500 kg.

Inicialmente, el tamaño muestral estuvo impuesto por el diseño metodológico del proyecto Europeo DRUID [58], según el cual, los países participantes deberían realizar, al menos, 3000 muestras con sus correspondientes cuestionarios válidos. Este tamaño muestral permitía para el conjunto de la población (participantes europeos), una precisión del 1\% con un intervalo de confianza del 95\% [IC 95\%]. Para el estudio español iniciado en 2008, el tamaño final de la muestra fue de 3302 conductores. A la vista de los resultados obtenidos en 2008, para el estudio de 2013 se estableció un máximo de 2944 conductores y finalmente se incluyeron 2932 cuestionarios válidos. Con esta muestra seguía manteniéndose la precisión del 1\% [IC 95\%].

Para estimar las prevalencias de consumo de sustancias por parte de los conductores españoles, se diseñó un estudio transversal, cada conductor era muestreado en una sola ocasión y con igual metodología, se llevó a cabo el posterior muestreo en 2013.

Se realizó un muestreo estratificado con un total de 128 puntos de control y teniendo en cuenta la temporalidad de los controles para la toma de muestras:

\section{Distribución geográfica}

- 4 Áreas: "Cantábrica", "Norte", "Mediterránea", y "Sur".

- 8 zonas por área: 32 zonas (16 zonas urbanas y 16 interurbanas).

- 4 Puntos de control por zona. 


\section{Cronograma: temporalidad de los controles de tráfico}

Se establecieron cuatro categorías o periodos en la semana en relación a posibles diferencias en la prevalencia del consumo de drogas:

- Periodo a (laborable "diurno"): L, M, X, J, V de 7:00 a 23:59 horas.

- Periodo b (laborable "madrugada"): M, X, J y V de 24:00 a 6:59 horas.

- Periodo c (fin de semana "diurno"): S, D y día festivo de 7:00 a 23:59 horas.

- Periodo d (fin de semana "madrugada"): S, D, L y festivo de 24:00 a 6:59 horas.

La obtención de las muestras y la cumplimentación de los datos de las entrevistas a los conductores se realizaron durante los controles que realizan las policías con competencias en tráfico.

El trabajo de campo realizó durante un periodo de 48 semanas, desde el 26 de septiembre de 2008 hasta el 24 de agosto de 2009. En el año 2013 se realizó del 20 de mayo al 18 de junio y del 11 al 28 de noviembre.

La muestra final fue ponderada (separadamente para cada estudio) teniendo en cuenta la intensidad del tráfico en los diferentes puntos de control.

\section{Prueba de cribado}

A todos los conductores detenidos en cada punto de control se les sometió inicialmente a una determinación cualitativa de drogas en fluido oral, mediante el dispositivo Draeger 5000 DrugTest $($, que permite la determinación "in situ" para fines de cribado de seis tipos de sustancias: Cocaína, anfetaminas, cannabis (THC), opioides, metanfetaminas y benzodiacepinas.

Al igual que la prueba de alcoholemia, la prueba de cribado tuvo carácter obligatorio para todos los conductores participantes. En esta primera prueba se ha tomado para cada sustancia el siguiente límite analítico: Opiáceos (Morfina), $20 \mathrm{ng} / \mathrm{ml}$; Anfetamina (D-anfetamina), $50 \mathrm{ng} / \mathrm{ml}$; Metanfetamina (DMetanfetamina), $35 \mathrm{ng} / \mathrm{ml}$; Cocaína (Cocaína), 20 ng/ml; THC (Delta-9-THC), $25 \mathrm{ng} / \mathrm{ml}$; Benzodiazepinas (Diazepam), $15 \mathrm{ng} / \mathrm{ml}$. 


\section{Prueba evidencial}

Las muestras de fluido oral se recogieron con los dispositivos Statsure Saliva Sampler ${ }^{\circledR}$ en 2008 y con Quantisal ${ }^{\mathrm{TM}}$ en 2013. Esto dispositivos están compuesto por una lengüeta de tejido almohadillado absorbente unida a una varilla aplicadora con un medidor de volumen (se precisa $1 \mathrm{ml}$ de saliva para realizar los análisis), que se inserta en un tubo que contiene un buffer estándar, una vez tomada la muestra.

Las muestras de saliva fueron enviadas al laboratorio en un contenedor específico (termoviales con gel congelado TMVL LN $®$ y cajas isotermas ATP650 () ) que las mantiene a una temperatura entre 2 a $8^{\circ} \mathrm{C}$. El envío se realizó dentro de las 36 horas siguientes a la muestra, salvo los controles desarrollados de jueves a domingo. En estos casos, la muestra fue congelada a $-20^{\circ} \mathrm{C}$ y enviada el martes hábil siguiente al laboratorio. El trasporte se realizó al laboratorio mediante la empresa de trasporte contratada, y en todo momento se mantuvo la correspondiente cadena de custodia de las muestras.

Se consideró que un conductor era positivo a una droga o a un medicamento, cuando la concentración de dicha sustancia en el fluido oral superaba la cantidad indicada en la tabla 3. En el caso del alcohol se consideró positivo, si la concentración de alcohol en el aire espirado superaba los $0.05 \mathrm{mg} / \mathrm{l}$.

\section{Análisis estadístico}

Las prevalencias se presentan como porcentajes con su Intervalo de Confianza al 95\%. Las comparaciones entre grupos (separadamente en cada uno de los estudios 2008 y 2013) se han establecido mediante la prueba de la Chi-cuadrado de Pearson. En el año 2008 se ha analizado la correlación entre las variables casos positivos y edad (correlación lineal y/o polinómica). Con el fin de determinar si ciertos aspectos sociodemográficos y pautas de conducción, se relacionan con una mayor o menor presencia de casos positivos de alcohol, drogas y medicamentos en los conductores españoles, se han planteado diversos modelos de regresión logística. Se presentan los valores de OR y su intervalo de confianza al $95 \%$.

La comparación entre los estudios de 2008 y 2013 para la prevalencia de las diferentes agrupaciones de sustancias se ha realizado mediante el test $Z$. 
Para todas las pruebas la significación estadística se ha establecido en valores de $P<0.05$.

Para el análisis de los datos se ha utilizado el programa SPSS v.18 y v.19.

Tabla 3 Sustancias analizadas y puntos de corte (cut-offs) para resultado positivo, de acuerdo con el criterio del proyecto europeo DRUID [58].

\begin{tabular}{|c|c|c|}
\hline Sustancia & Fluido Oral cut-off $(\mathrm{ng} / \mathrm{ml})$ & Aire espirado $(\mathrm{mg} / \mathrm{l})$ \\
\hline Etanol & --- & 0.05 \\
\hline 6-Acetilmorfina & 16.0 & \\
\hline Alprazolam & 3.5 & \\
\hline Amfetamina & 360.0 & \\
\hline Benzoilecgonina & 95.0 & \\
\hline Clonazepam & 1.7 & \\
\hline Cocaína & 170.0 & \\
\hline Codeína & 94.0 & \\
\hline Diazepam & 5.0 & \\
\hline Flunitrazepam & 1.0 & \\
\hline Lorazepam & 1.1 & \\
\hline MDA & 220.0 & \\
\hline MDEA & 270.0 & \\
\hline MDMA & 270.0 & \\
\hline Metadona & 22.0 & \\
\hline Metanfetamina & 410.0 & \\
\hline Morfina & 95.0 & \\
\hline Nordiazepam & 1.1 & \\
\hline Oxazepam & 13.0 & \\
\hline THC & 27.0 & \\
\hline Zolpidem & 10.0 & \\
\hline Zopiclona & 25.0 & \\
\hline Tramadol & 480.0 & \\
\hline 7-Amino-clonazepam & 3.1 & \\
\hline 7-Amino-flunitrazepam & 1.0 & \\
\hline
\end{tabular}




\section{Aspectos éticos y legales}

El estudio fue informado favorablemente por el Comité Ético de Investigación de la Universidad de Valladolid el 31 de Enero de 2007. Dicho comité aprobó el diseño y los objetivos del estudio, así como los documentos de "Información al participante" y "Consentimiento Informado", a cumplimentar por los conductores que voluntariamente se sometieran a la obtención y entrega, en su caso, de la muestra biológica de fluido oral con fines exclusivos de investigación. Para los supuestos de resultado negativo en los test de cribado, estos documentos garantizaron al participante la finalidad investigadora de las muestras analizadas, su uso confidencial y la no utilización con fines sancionadores.

\section{Rechazo a participar y casos no incluidos en el estudio}

Como se ha comentado anteriormente, los conductores tienen la obligación de someterse tanto a la prueba de alcohol como a la prueba de drogas drogas, regulada según la normativa administrativa y penal vigente. Sin embargo, la participación en el estudio era voluntaria y los participantes firmaron el correspondiente consentimiento informado.

Cumpliendo los criterios de inclusión y exclusión del estudio, se ha considerado rechazo en el reclutamiento:

a. La negativa a someterse al control

b. El no consentimiento a la cesión de muestra de fluido oral a efectos de investigación, en el caso de haber realizado la prueba de cribado con resultado de negativa a la misma.

c. Cuando no se ha podido obtener muestra por evidente falta de salivación.

\section{Evolución del consumo}

Una vez realizada la ponderación por intensidad del tráfico, no existen diferencias significativas en la distribución de las muestras de 2008 y de 2013 respecto al área $\quad\left(X^{2}{ }_{3}=0.02 ; \mathrm{p}>0.05\right)$, el tipo de vía $\left(X^{2}=0.02 ; \mathrm{p}>0.05\right)$ o el periodo semanal/horario $\left(\mathrm{X}_{3}{ }_{3}=0.06 ; \mathrm{p}>0.05\right)$. En la Figura 4 se presenta un perfil comparativo de ambas muestras una vez realizada la ponderación por la intensidad del tráfico. Por lo tanto y como en el análisis de cribado se utilizó el mismo dispositivo en los estudios de 2008 y 2013 (DrugTest 5000, con las 
mismas especificaciones técnicas), parece adecuado proceder a realizar directamente la comparación de los resultados del cribado obtenidos en ambos estudios.

La diferencia fundamental con el estudio del año 2008 fue que solamente se realizaron análisis de confirmación en laboratorio, a aquellos conductores que resultaron positivos a alguna sustancia en el test de cribado. Como consecuencia, hubo que analizar el efecto que esto suponía sobre los análisis de confirmación. Este efecto se analizó sobre la muestra de 2008 ya que en 2013 no se analizó la saliva de aquellos conductores que resultaron negativos en el cribado.

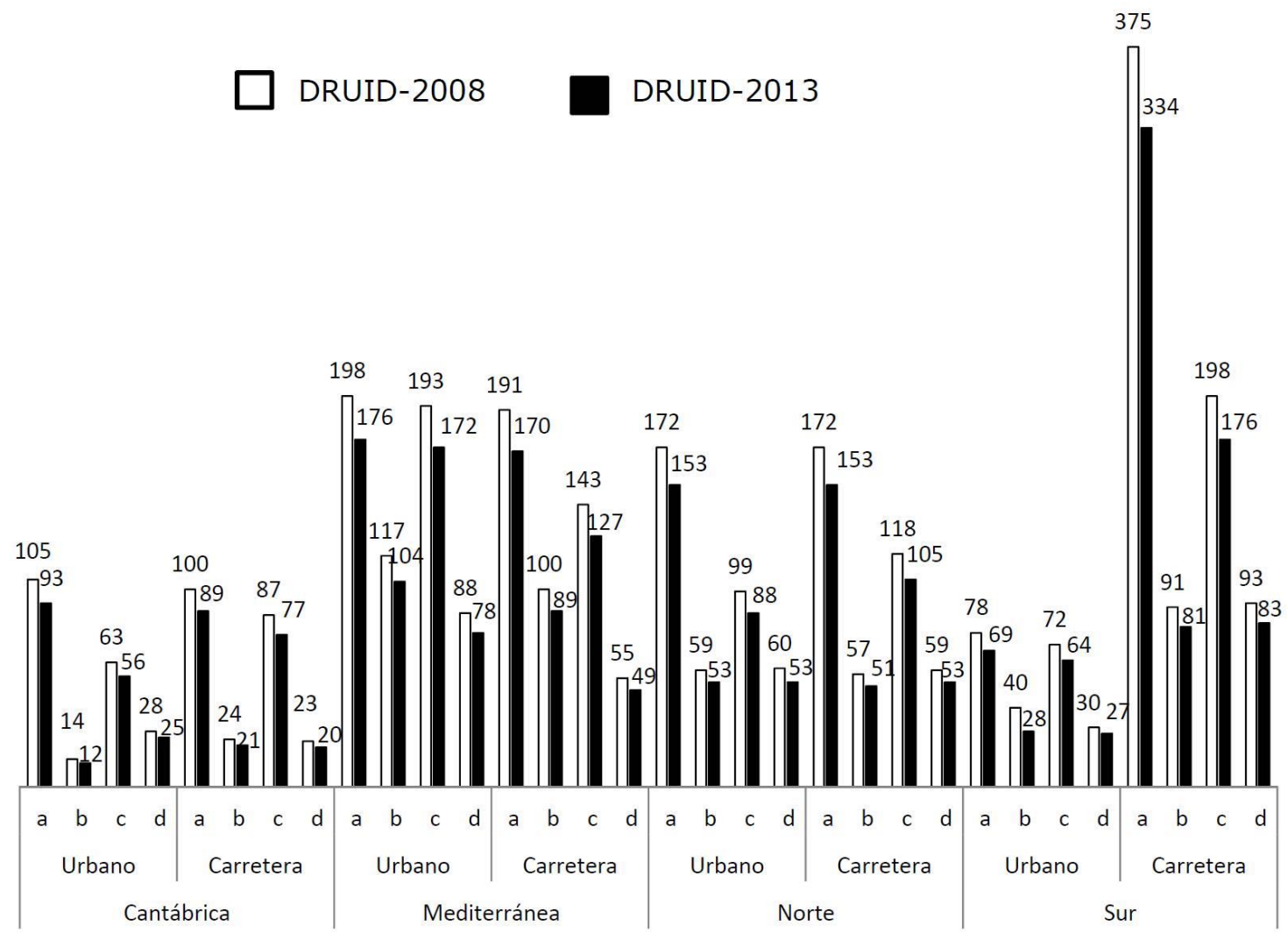

Figura 4 Distribución de las muestras para los proyectos DRUID-2008 y DRUID-2013 una vez realizada la ponderación por intensidad del tráfico 


\subsubsection{Resultados}

\section{Prevalencias en el estudio de 2008}

En 2008, el $17 \%$ de los conductores resultaron positivos en alguna de las sustancias analizadas (95\% IC, 15.5-18.2). El 6.6\% (5.7-7.4) de los conductores resultaron positivos en alcohol en aire espirado (>0.05 mg/l), el 11.5\% (9.8-12.0) en alguna droga ilegal, y el $2.0 \%(1.5-2.4)$ resultaron positivos en alguno de los medicamentos analizados. Es necerario advertir que alguno de los conductores resultaron positivos en más de una sustancia (Figura 5).

En el $70 \%$ de los casos en los que se detectó alcohol, solamente se detectó esta sustancia. El $31 \%$ de los positivos en alcohol lo fueron por encima del límite legal en España (0.25 mg de etanol/I de aire espirado).

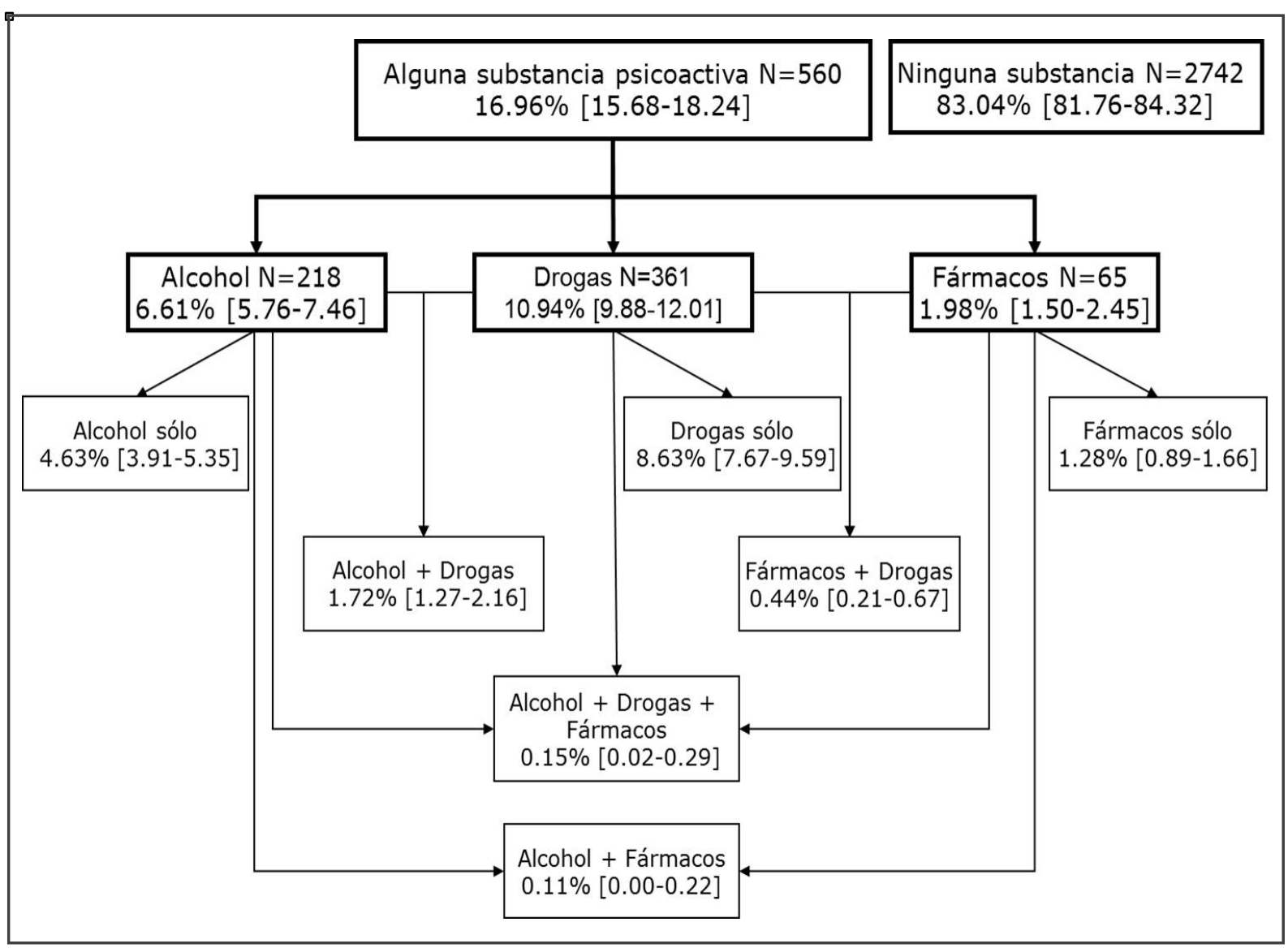

Figura 5 DRUID 2008. Casos positivos en alcohol, drogas ilegales y medicamentos en los conductores españoles (puntos de corte indicados en la Tabla 3) 
Conducir después del consumo de sustancias psicoactivas es un hecho frecuente en España, alcanzando el $17 \%$ de los conductores españoles. Casi un $11 \%$ de los conductores conducen tras haber consumido otras sustancias psicoactivas distintas al alcohol; cannabis y cocaína explican la mayor parte de los casos positivos a drogas. La Figura 6, muestra un resumen de los resultados obtenidos, e ilustraba un artículo publicado en la revista Tráfico y Seguridad Vial, editada por la Dirección General de Tráfico [59].

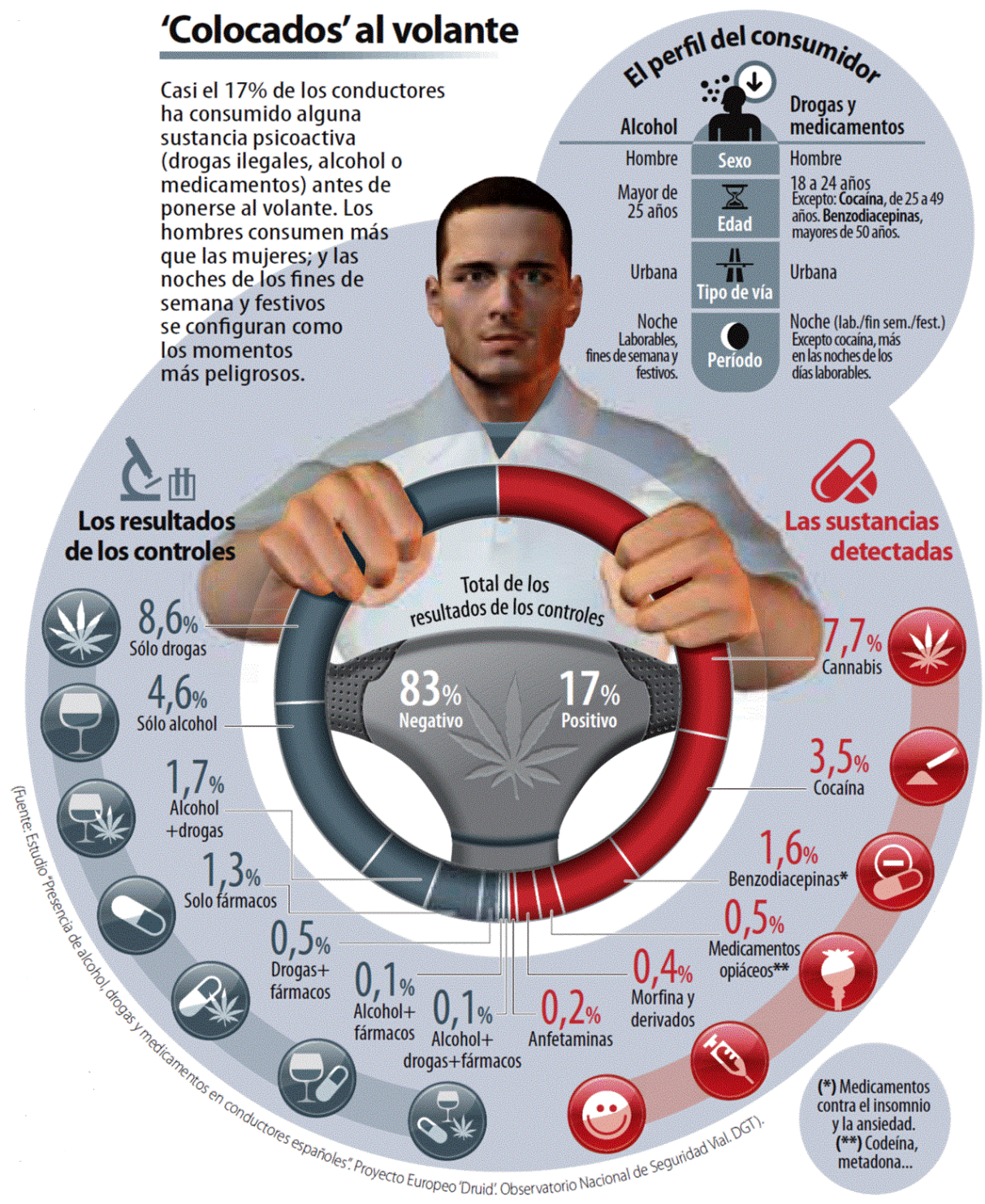

Figura 6 Prevalencias de sustancias en conductores. Resumen de los resultados publicados en la revista Tráfico y seguridad vial N. 212. 
La frecuencia de casos positivos en "alguna sustancia" ( $y=$ frecuencia) disminuye a medida que aumenta la edad de los conductores ( $x=$ edad en años), pudiéndose establecer una correlación lineal $\left(y=27.73-0.29 x ; \quad r^{2}=0.27\right.$; $p<0.0001)$.

Para el alcohol se observan diferentes tendencias con la edad, dependiendo de si consideramos todos los casos positivos en alcohol $\left(y=4.57+0.06 x ; r^{2}=0.06\right.$; $p>0.05)$, los positivos en alcohol solamente $\left(y=1.47+0.095 x ; r^{2}=0.14 ; F=22.00\right.$; $p<0.01)$ o los positivos en alcohol+drogas $\left(y=3.50+0.057 x ; \quad r^{2}=0.67 ;\right.$ $p<0.0001)$.

Para el THC, cocaína y benzodiacepinas, las tendencias pueden ajustarse a polinomios de tercer grado, siguiendo tres curvas bien diferenciadas. Mientras que para el THC los mayores porcentajes de consumo se observan entre los más jóvenes, la prevalencia de benzodiacepinas en conductores aumenta con la edad. En el caso de la cocaína, el máximo se sitúa entre los 25 y 35 años (ver Figura 4 en el Capítulo 2).

Mediante diferentes modelos de regresión logística se pudo observar que la probabilidad de encontrar casos positivos a cualquier sustancia disminuye al aumentar la edad $(\mathrm{OR}=0.98)$, es mayor entre los hombres que entre las mujeres $(O R=2.77)$ y también superior, en las carreteras urbanas respecto de las interurbanas $(\mathrm{OR}=1.80)$. Respecto a los periodos horarios y semanales establecidos, la probabilidad más baja ocurre los días laborables en el periodo diurno (de 7:00 a 23:59), siendo la más alta, los fines de semana en el periodo nocturno $(O R=3.29)$, seguida de los días laborables por la noche $(O R=2.81)$ y de los fines de semana en periodo diurno $(O R=1.68)$.

\section{Efecto del cambio de metodología en la estimación de prevalencias en el año 2008}

Como se ha señalado anteriormente, hubo que volver a realizar el análisis estadístico del año 2008, considerando los casos que fueron negativos en el cribado, como definitivamente negativos en el análisis de confirmación. En la tabla 4 puede observarse que esto supone unos cambios significativos en la distribución de las prevalencias $\left(\mathrm{X}_{8}{ }_{8}=38.09 ; \mathrm{p}<0.0001\right)$ del año 2008. Por lo 
tanto, no serían directamente comparables las prevalencias publicadas pare el estudio de 2008 [4], con las obtenidas para la muestra de 2013. Como consecuencia, en los análisis de confirmación la comparación se establecerá con los resultados obtenidos al aplicar las condiciones del estudio de 2013 a ambos estudios: Los negativos en la prueba de cribado serán considerados negativos en la prueba de confirmación en ambos estudios.

Tabla 4 Comparación de resultados para el estudio llevado a cabo en 2008: con la metodología de 2008 (resultados publicados [4]) y la nueva metodología

\begin{tabular}{lllll}
\hline & \multicolumn{3}{l}{ Positivos DRUID-2008 } \\
\cline { 2 - 5 } & $\mathrm{N}$ & $\%$ & $\mathrm{~N}$ & $\%$ \\
\hline Ninguna sustancia & 2743 & 83.1 & 2838 & 86.0 \\
Cannabis & 216 & 6.5 & 174 & 5.3 \\
Cocaína & 54 & 1.6 & 42 & 1.3 \\
Anfetaminas & 3 & 0.1 & 2 & 0.1 \\
Opiáceos & 6 & 0.2 & 5 & 0.1 \\
Alcohol >0.05 & 153 & 4.6 & 162 & 4.9 \\
Benzodiacepinas & 42 & 1.3 & 5 & 0.1 \\
Varias drogas & 20 & 0.6 & 18 & 0.5 \\
Alcohol+drogas & 65 & 2.0 & 56 & 1.7 \\
\hline & & $\mathrm{X}^{2}=38.0906 ; \mathrm{p}<0.0001$ & 14.0 \\
\hline Alguna sustancia & 559 & 16.9 & 464 & \\
\hline
\end{tabular}

\section{Evolución del consumo entre los años 2008 y 2013}

Resultados de la prueba de cribado: En 2013, el $12.07 \%$ de las pruebas de cribado realizadas en carretera para el estudio resultaron positivas en alguna sustancia, lo que supone un descenso del $4.27 \%$ respecto a los resultados de 2008 (16.34\%, Tabla 5). Se observa un descenso significativo, tanto en los casos que resultaron positivos solamente a alcohol como en los que solamente resultaron positivos en el cribado de drogas, pero este descenso es aún más significativo para los casos positivos en alcohol+drogas (de $2.03 \%$ en 2008 a $0.81 \%$ en 2013). 
Resultados del análisis de confirmación en laboratorio ${ }^{1}$ : En el estudio de 2013 se confirmó la presencia de sustancias en el $9.34 \%$ de los conductores que participaron en el estudio. Respecto del $14.05 \%$ de resultados positivos observados en el estudio de 2008, el resultado de 2013 supone un descenso del $4.71 \%$ en la prevalencia de casos positivos. Analizando las posibles combinaciones de sustancias, entre 2008 y 2013 se observa un descenso significativo en los casos positivos a, solamente alcohol y a alcohol+drogas (Tabla 5). También se observa un descenso significativo en los casos positivos solamente a drogas (2008: 6.93\% [6.07-7.80]; 2013: 4.87\% [4.09-5.65]; $\mathrm{p}<0.01$ ), debido a la menor prevalencia de cannabis observada en 2013 (Tabla $5)$.

\footnotetext{
${ }^{1}$ Los resultados del estudio de 2008 referidos en este apartado difieren de los de las figuras 5 y 6 por haber sido calculados con la metodología del estudio de 2013: Todos los negativos en el cribado se consideraron resultados negativos a todos los efectos, en la prueba de confirmación.
} 


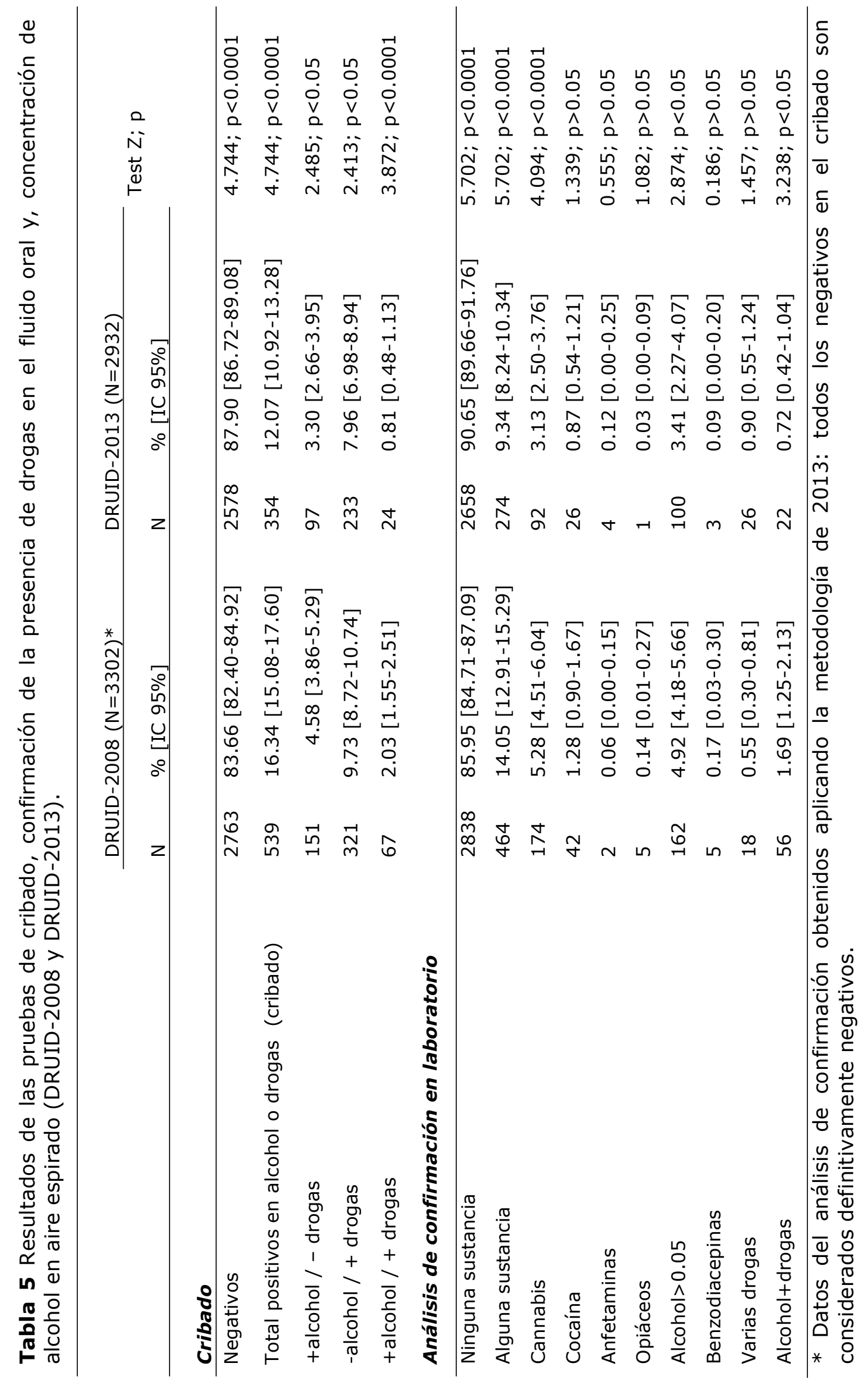




\section{Conclusiones}

Prevalencia de sustancias en conductores en 2008:

- Conducir después del consumo de sustancias psicoactivas es un hecho frecuente en España, alcanzando el 17\% de los conductores españoles.

- Casi un $11 \%$ de conductores conducen tras haber consumido otras sustancias psicoactivas distintas al alcohol.

- Cannabis y cocaína explican la mayor parte de los casos positivos a drogas.

- En lo que respecta a las drogas de comercio ilegal, la prevalencia disminuye a medida que aumenta la edad de los conductores, es más frecuente en varones, en vías urbanas y durante la madrugada, tanto en fin de semana como en días laborales.

Evolución del consumo:

- Respecto de 2008, en 2013 se observa un descenso superior al $4 \%$ en la prevalencia de casos positivos a sustancias en los conductores españoles (4.2\% o 4.7\% según consideremos el cribado o el análisis de confirmación).

- A pesar de este descenso, la prevalencia de alcohol (4.16\%), drogas $(5.79 \%)$ y medicamentos $(0.29 \%)$ en los conductores españoles, es aún elevada.

- La realización de controles aleatorios de carretera, con análisis de la presencia de drogas en saliva además de los controles de alcohol en aire espirado, es la causa disuasoria más probable para explicar este descenso en la prevalencia de sustancias en conductores. 



\subsection{El pictograma español sobre medicamentos y conducción}

\subsubsection{Metodología}

La población diana era la población española no institucionalizada, tanto conductores como no conductores, de 18 años o más, residentes en Valladolid que eran usuarios del Sistema Nacional de Salud en el momento de realización de la encuesta.

Los cuestionarios se cumplimentaron mediante entrevistas personales realizadas aleatoriamente entre las personas: i) que acudían a consultas de atención primaria en 6 centros diferentes, ii) que acudían a la consulta de preanestesia del Hospital Clínico Universitario de Valladolid y, iii) personas que acudían a comprar a 5 farmacias diferentes realizadas por entrevistadores experimentados y específicamente formados para este proyecto, lo que facilitó y simplificó en gran medida la participación de los entrevistados. El tamaño de la muestra se estableció inicialmente en 900 cuestionarios válidos (300 en cada grupo). Finalmente se realizaron un total de 1385 cuestionarios válidos, entre mayo y octubre de 2010.

El estudio fue aprobado por el Comité Ético de Investigación Clínica de la Facultad de Medicina de la Universidad de Valladolid, Ref. 2010/30.

\section{Variables consideradas en el estudio}

Variables sociodemográficas. i) Sexo (hombre/mujer), ii) Edad (años), iii) Permiso de conducir (si/no), iv) km conducidos al año, y v) Nivel de estudios (No terminó educación primaria/ primarios/ secundaria/ bachillerato/ diplomatura o licenciatura).

Conocimiento previo sobre medicamentos y conducción. Se preguntaba al entrevistado si sabía que algunas medicinas pueden influir en la capacidad de conducir (si/no);

Comprensión del pictograma. Al entrevistado se le presenta el envase de un medicamento real con pictograma y se le pregunta: "En su opinión, ¿qué 
significado tiene para Vd. el pictograma sobre conducción?" Las respuestas se recogen de forma abierta y posteriormente se agrupan en 5 categorías siguiendo el modelo propuesto en la norma ISO 9186-1:2007: 1 = Correcta; 2 = Errónea; 3 = Errónea y con el significado opuesto a lo que se desea transmitir con el pictograma; 4 = La respuesta referida es "No sé"; $5=$ Sin respuesta. Los porcentajes se calculan sobre el total de respuestas en las categorías de 1 a 4 . Se considera que el significado opuesto al propósito del pictograma es "no tomar, o dejar de tomar, el medicamento si se conduce".

Riesgo percibido para la conducción ante la observación del pictograma sobre medicamentos y conducción. Al entrevistado se le pregunta: "A la vista del pictograma ¿Cómo evaluaría usted el grado de influencia de este medicamento en la conducción?, es decir, el riesgo que tiene tomar ese medicamento y conducir vehículos". Se ofrecen cuatro opciones de respuesta (alto riesgo/ riesgo moderado/ bajo riesgo/ sin riesgo/ no lo sé).

Posible influencia del pictograma en la actitud del conductor - cambio en la frecuencia de conducción. El entrevistador expone: "Supongamos que a usted le prescriben este medicamento en el que aparece el pictograma sobre conducción en el envase ¿Con qué frecuencia conduciría en el periodo en que estuviese tomando el medicamento?" Las opciones de respuesta eran "Con la misma frecuencia", "Menos frecuentemente", "Bastante menos frecuentemente", "Casi no conduciría" y "No conduciría". Para algunos análisis (regresión logística) esta variable se recodificó en dicotómica, "Conduce con la misma frecuencia"/ "Cambia su frecuencia de conducción".

Valoración del pictograma: Se puntúan por separado, de 1 a 10 puntos (escala Likert de 10 puntos), cuatro aspectos del pictograma mostrado: "utilidad" (1=innecesario a 10=útil), "información" (1=no informativo a 10=Informativo), "comprensibilidad" (1=incomprensible a 10=comprensible), y "sencillez" ( $1=$ complejo a $10=$ sencillo). Además se pidió a los entrevistados una valoración global del pictograma mostrado, puntuando de 1 (mínimo) a 10 (máximo). 


\section{Análisis estadístico}

Para las variables continuas se presentan las medias con su desviación estándar (media $\pm \mathrm{DE})$ y las frecuencias para las variables categóricas. Las comparaciones entre dos grupos independientes se han realizado, para las variables continuas, mediante la t de Student y en el caso de las variables categóricas se ha utilizado el test de la chi-cuadrado de Pearson.

Se utilizó la regresión logística para determinar las variables sociodemográficas que influyen en la comprensión del pictograma. La variable de 5 categorías (según ISO 9186-1:2007) se recodificó como variable dicotómica, con el fin de comparar el grupo que asignó respuestas correctas frente al resto. En el análisis de la comprensión del pictograma se ha considerado el efecto de las covariables, edad, sexo, nivel educativo, carné de conducir, tener conocimiento de que algunos medicamentos pueden influir sobre la conducción y conocimiento previo del pictograma.

La regresión logística también se utilizó para evaluar el impacto del pictograma en la actitud de los conductores, analizando el posible cambio en la frecuencia de conducción. Para ello, las 4 categorías de la variable que implicaron una disminución en la frecuencia de conducción se agruparon en una sola, obteniendo así una variable dicotómica: "cambia la frecuencia de conducción sí/no". La influencia de los factores antes mencionados se controló y, además, por los miles de kilómetros recorridos al año y la diferente percepción del riesgo que supone conducir, habiendo tomado un medicamento con el pictograma en su envase.

Con coeficiente alfa de Cronbach se analizó la fiabilidad de la escala Likert utilizada en la valoración del pictograma. La influencia de las variables, edad (en rangos: <25; 25-34; 35-44; 55-64 y >64 años), sexo, nivel educativo y posesión de carné de conducir en la valoración de los distintos aspectos del pictograma y en la puntuación final, se ha analizado utilizando tests no paramétricos (U de Mann-Whitney o Kruskal-Wallis).

En todos los tests, el nivel de significación estadística se ha establecido en $\mathrm{p}<0.05$ con un intervalo de confianza del 95\% [95\% IC]. Para el análisis de 
datos se utilizó el paquete estadístico SPSS v. 18 con licencia para la Universidad de Valladolid.

\subsubsection{Resultados}

Se realizaron 1385 entrevistas válidas, el 47.7\% a hombres $(n=660)$ y el $52.3 \%$ a mujeres $(n=725)$. La media de edad era de $50.50 \pm 15.55$ años observándose diferencias significativas entre hombres y mujeres (54.25 \pm 15.70 años, los hombres y $47.09 \pm 14.61$ años las mujeres; $t=8.758$ [5.553; 8.759]; $p<0.0001)$. El $79.3 \%$ tenían carnet de conducir $(n=1098)$ y el $20.7 \%$ no $(n=287)$.

El $93.9 \%$ de los entrevistados (el $95.4 \%$ de los conductores y el $88.5 \%$ de los no conductores, $\left.X^{2}=18.76 ; p<0.05\right)$ sabían que algunos medicamentos podían afectar a su capacidad de conducir.

De los 1363 entrevistados que respondieron a la pregunta "En su opinión ¿Qué significado tiene para usted el pictograma sobre conducción?" El $85.7 \%$ (el $90.5 \%$ de los conductores y el $67.4 \%$ de los no conductores; $\left.X_{3}{ }^{2}=115.24 ; p<0.0001\right)$ relacionaron correctamente el símbolo con los posibles efectos del medicamento sobre la conducción. La probabilidad de una correcta interpretación disminuía a medida que aumentaba la edad de los entrevistados, OR $=0.969$ [0.957-0.980] y era mayor cuanto mayor era el nivel educativo $\mathrm{OR}=1.213[1.047-1.405]$. También era mayor entre aquellos que tenían carnet de conducir $\mathrm{OR}=3.268$ [2.315-4.630] y entre los que sabían que algunas medicinas podían afectar a la capacidad para conducir $O R=2.004$ [1.163-3.448].

Después de observar el pictograma impreso sobre el envase del medicamento, el $48.3 \%$ de los entrevistados (el $45.2 \%$ de los conductores y el $60.1 \%$ de los no conductores) consideró que tomar el medicamento podía suponer un riesgo "alto" para conducir, el 33.9\% un riesgo "moderado", el 4.5\% un riesgo "bajo" y el solo el $0.8 \%$ lo consideró "sin riesgo". Se observan diferencias significativas en la percepción del riesgo entre conductores y no conductores $\left(X_{4}^{2}=41.77 ; p<0.0001\right)$. 
La presencia de este pictograma en el envase de los medicamentos podría ejercer una influencia en la actitud de los conductores. Solamente un $16.1 \%$ de los conductores no reduciría su frecuencia de conducción si le prescribieran un medicamento con pictograma. Según las respuestas referidas, sería más probable reducir la frecuencia de conducción a medida que aumenta la edad del conductor $\mathrm{OR}=1.022[1.008-1.037] \mathrm{y}$ menos probable entre los conductores que conducen más kilómetros al año $\mathrm{OR}=0.989$ [0.983-0.994]. La percepción del riesgo es un factor muy influyente en esta actitud. Tomando como referencia aquellos que consideraron que tomar el medicamento no suponía "ningún riesgo" o un riesgo "bajo" para conducir, sería más probable reducir la frecuencia de conducción entre aquellos que consideraron que el riesgo era "muy alto" $\mathrm{OR}=16.500$ [8.340-32.645] o moderado OR = 3.557 [1.923-6.577].

El pictograma fue bien valorado como "útil", "informativo", "comprensible" y "sencillo". El coeficiente alfa de Cronbach de 0.837 indica una elevada fiabilidad para la escala Likert de cuatro elementos utilizada para evaluar los cuatro aspectos mencionados. La media $( \pm D E)$ en la valoración global fue de $7.98 \pm 1.58$ puntos. 


\section{Conclusiones}

- La mayor parte de los entrevistados (el 93.9\%) sabía que algunos medicamentos podían afectar a su capacidad de conducir.

- El pictograma español sobre medicamentos y conducción es comprendido por la mayoría de los entrevistados. El $85.7 \%$ (el $90.5 \%$ de los conductores y el $67.4 \%$ de los no conductores) relacionaron correctamente el símbolo con los posibles efectos del medicamento sobre la conducción.

- Con una puntuación global media de 7.98 1.58 puntos, este pictograma es considerado "útil", "informativo", "comprensible" y "sencillo" por los usuarios del Sistema Nacional de Salud.

- Su presencia en el envase de los medicamentos podría ejercer una influencia en la actitud de los conductores: el $83.9 \%$ reduciría su frecuencia de conducción si le prescribieran un medicamento con pictograma.

Este pictograma puede considerarse una herramienta para mejorar los procedimientos de prescripción y dispensación de medicamentos que afecten a la capacidad de conducir y también, como un instrumento para concienciar a los pacientes acerca del papel que los medicamentos pueden jugar en la seguridad vial. 


\subsection{Relación entre los signos de deterioro en conductores y las concentraciones de THC en su fluido oral}

\subsubsection{Metodología}

Los datos analizados en este trabajo han sido extraídos de la base de datos española generada para el Proyecto Europeo DRUID. Los datos se obtuvieron en controles de carretera realizados desde finales de julio de 2008 hasta agosto de 2009 [4].

En los controles de carretera, los conductores fueron seleccionados de forma aleatoria. El oficial de policía realizó una corta entrevista al conductor recogiendo datos acerca de sus hábitos de bebida, su posible historial en el consumo de sustancias y su consumo reciente. Los datos de los participantes en el estudio se recogieron de forma anónima e incluyeron: fecha y hora del control, edad, género, tipo de vehículo, tipo de carretera (urbana/rural), presencia o no de signos de deterioro (31 signos observables, posibles indicadores de consumo de sustancias) auto-informe de consumo de drogas y, para los que rechazaron participar en el estudio, la razón del rechazo. Los participantes en el estudio firmaron el correspondiente consentimiento informado.

Todos los conductores detenidos en los controles (rechazaran o no tomar parte en el estudio) realizaron una prueba de alcoholemia en aire espirado y un test de dogas en fluido oral "In-Situ". Ambos test tuvieron carácter obligatorio. Tanto la petición voluntaria para participar en el estudio, como la toma de muestra de fluido oral para el análisis de confirmación, se realizaron mientras los conductores esperaban el resultado de la prueba obligatoria "In-Situ". La acertada secuencia en la recogida de información y realización de las pruebas derivó en una muy baja tasa de rechazo [60].

Los agentes de tráfico recibieron formación específica para la observación de los signos de deterioro. Los 31 signos observados comprenden siete "signos de actitud", cuatro de "aspecto corporal", cinco de "expresión facial", tres "signos del habla", cuatro "signos de coordinación" y ocho "signos oculares". El informe era cumplimentado por el agente de tráfico. Una batería de tests similar, 
desarrollado en Holanda por SMOV (Institute for Road Safety Research) ya fue utilizada en el proyecto IMMORTAL [61] aunque para este proyecto se añadieron añadido algunos signos. La validación del método de screening para detectar conductores bajo los efectos de las sustancias, basado en la observación de los signos de deterioro, ha sido establecida por comparación con los resultados obtenidos en el análisis del fluido oral de los conductores.

Cada sustancia individual se consideró presente en la muestra, si fue detectada en el análisis de confirmación, aunque fuera a nivel de trazas (concentración inferior al límite de cuantificación). Este criterio se adoptó para garantizar, en la medida de lo posible, que los signos observados no correspondían a efectos residuales debidos a la ingesta de otra sustancia distinta de la que constituye, en cada caso, nuestro objetivo de análisis. En el presente estudio se incluyeron 2632 casos seleccionados a partir de una muestra representativa de 3302 conductores. La muestra incluye 253 casos positivos solamente en THC y 32 casos positivos en alcohol más THC pero negativos en cualquier otra droga. Estos casos se compararon con 201 conductores positivos etanol (solo etanol) y 2146 conductores negativos en drogas en su fluido oral y también negativos en alcohol en aire espirado. El resto de los conductores (670) fueron excluidos del estudio, por resultar positivos a otras sustancias o combinaciones de sustancias fuera del objeto de este estudio (639) o por no disponer de datos acerca de la presencia o ausencia de signos de deterioro (31 casos perdidos).

En general, el grupo en el que no se detectaron sustancias se utilizó como grupo de referencia en la comparación de los otros grupos en los que se detectaron sustancias (THC solo, alcohol solo, THC+alcohol), aunque en algunos análisis se establecieron comparaciones con el grupo en el que se detectó solamente alcohol u otros grupos.

\section{Análisis estadístico}

Se consideró positivo en el test de signos, aquel conductor en el que se observó uno o más de los 31 signos de deterioro del listado (Tabla 6). La fiabilidad del test observacional (basado en los signos de deterioro observados) se evaluó separadamente en cada grupo de conductores, calculando su sensibilidad, especificidad, valor predictivo positivo (VPP), valor predictivo negativo (VPN) y 
valor global de la prueba o eficiencia de la prueba (VG), de acuerdo a los resultados obtenidos en el análisis del fluido oral realizado en el laboratorio y, para el alcohol, de acuerdo al análisis de alcohol realizado en aire espirado. Para este propósito se establecerán como puntos de corte para considerar casos positivos: en el caso del THC, una concentración igual o superior a 27 ng de THC en fluido oral, y para el alcohol, una concentración $>0.25 \mathrm{mg}$ de etanol por litro de aire espirado.

La fiabilidad del test de detección de drogas "in-situ" (dispositivo Draëger 5000) se evaluó, al igual que el test de signos, por contraste con los resultados obtenidos en el análisis del fluido oral realizado en el laboratorio.

Con el fin de investigar la posible relación entre la concentración de THC en el fluido oral y los signos de deterioro observado (signos totales y/o diferentes grupos de signos y/o signos individuales) establecimos unos intervalos (rangos) de concentración. Este análisis se llevó a cabo con el grupo en el que se detectó solamente THC y los rangos se establecieron con los puntos de corte de una curva ROC (Receiver Operating Characteristic). Para la curva ROC se utilizaron los valores de concentración de THC en fluido oral y el test se consideró positivo, cuando se observó al menos un signo de deterioro. Los casos en los que la variable de cuantificación no presentaba un nivel numérico (45 casos registrados como $>200 \mathrm{ng} / \mathrm{ml} \mathrm{o}>400 \mathrm{ng} / \mathrm{ml}$ ) fueron excluidos del análisis inicialmente. Una vez establecidos los rangos, estos casos fueron incluidos en el rango superior en el superior ( $>100 \mathrm{ng} / \mathrm{ml}$ ). Una vez establecidos los rangos volvimos a calcular la curva ROC con la nueva variable de concentración en rangos, con el fin de observar el efecto de los casos no incluidos en el análisis previo (con la variable inicial de concentración). Determinamos así, para los casos positivos en THC solamente, cuatro intervalos de concentración de THC en el fluido oral: $\leq 3.00$ $\mathrm{ng} / \mathrm{ml} ; 3.01-25.00 \mathrm{ng} / \mathrm{ml} ; 25.01-100.00 \mathrm{ng} / \mathrm{ml}$ y $>100.00 \mathrm{ng} / \mathrm{ml}$.

En los resultados se presentan tablas de frecuencias para las variables categóricas y la media $\pm \mathrm{DE}$ (media \pm desviación estándar) para las variables continuas. El test de la chi-cuadrado de Pearson se utilizó con las variables categóricas, para las comparaciones entre grupos y para probar la validez del test observacional de signos a la hora de detectar deterioro debido a la presencia 
de sustancias. Además, se utilizó para valorar la asociación entre los rangos de concentración y el porcentaje de conductores en los que se presentan signos. Las medias de las variables continuas entre los diferentes grupos se contrastaron mediante el test de la t-Student.

La regresión logística por pasos sucesivos hacia adelante se utilizó para conocer el riesgo de ser observado con signos de deterioro cuando alcohol y/o THC se presentan en los diferentes rangos de concentración considerados. En el análisis se controlaron los posibles efectos de confusión de las variables, edad (variable continua), género (hombre/mujer), tipo de carretera (rural/urbana) y periodo (a: de lunes a viernes de 7:00 a 23:59 h; b: de martes a viernes de 0:00 a 6:59 h.; c: sábado/domingo/festivo de 7:00 a 23:59 h. y d: sábado/ domingo/ lunes/ festivo de 0:00 a 6:59 h.).

\subsubsection{Resultados}

Los conductores positivos en THC en su fluido oral presentaban más frecuentemente signos de deterioro (el 12.6\%) que los conductores que resultaron negativos (el $1.0 \%$; Tabla 6 ).

El porcentaje de conductores con signos es similar en el grupo con THC (12.6\%) $y$ en el grupo de alcohol solo (9.5\%; $\left.X^{2}=1.15 ; p>0.05\right)$. En el grupo de casos en los que se detectó alcohol y cannabis, la prevalencia de conductores en los que se observaron signos fue significativamente mayor $(28.1 \%)$ que en el grupo con solamente THC $\left(X^{2}=5.53 ; p<0.05\right)$. Los signos oculares son los más frecuentemente observados en el grupo de conductores con THC (9.9\%), seguidos de los signos de actitud (7.1\%).

En la Figura 7 se presentan los porcentajes de conductores en los que se observaron signos (cualquier signo y grupos de signos) para diferentes grupos de sustancias. Las diferencias significativas entre grupos se han señalado con un asterisco sobre la Figura 7. No se observa ninguna diferencia significativa entre el grupo de THC solo y el grupo de alcohol sólo, ni considerando los porcentajes de conductores que presentan "algún signo" ni tampoco, considerando los 
porcentajes para los diferentes grupos de signos. Para el grupo de casos en los que se detectó THC y alcohol simultáneamente, el porcentaje de conductores en los que se observan signos de deterioro se incrementa significativamente, tanto respecto del grupo en el que se detectó solamente alcohol, como del grupo en el que solamente se detectó THC. Esto se observa para "algún signo", para los "signos de actitud" y para los "signos oculares".

Se observa una correlación entre la concentración de THC en el fluido oral y algunos de los signos que los agentes de tráfico observaron en los conductores. La probabilidad de que en los conductores se observen signos oculares se incrementa significativamente, a concentraciones de THC $>3.0 \mathrm{ng} / \mathrm{ml}$ en su fluido oral. Concentraciones de THC $>25 \mathrm{ng} / \mathrm{ml}$ en el fluido oral se relacionan con una mayor probabilidad de presentar signos de comportamiento, signos faciales y signos del habla. Los análisis de regresión logística muestran una contribución independiente del alcohol y del cannabis a los efectos (signos) observados. Los efectos observados podrían describirse como la suma de los efectos del alcohol y del cannabis cuando éstos se consumen separadamente.

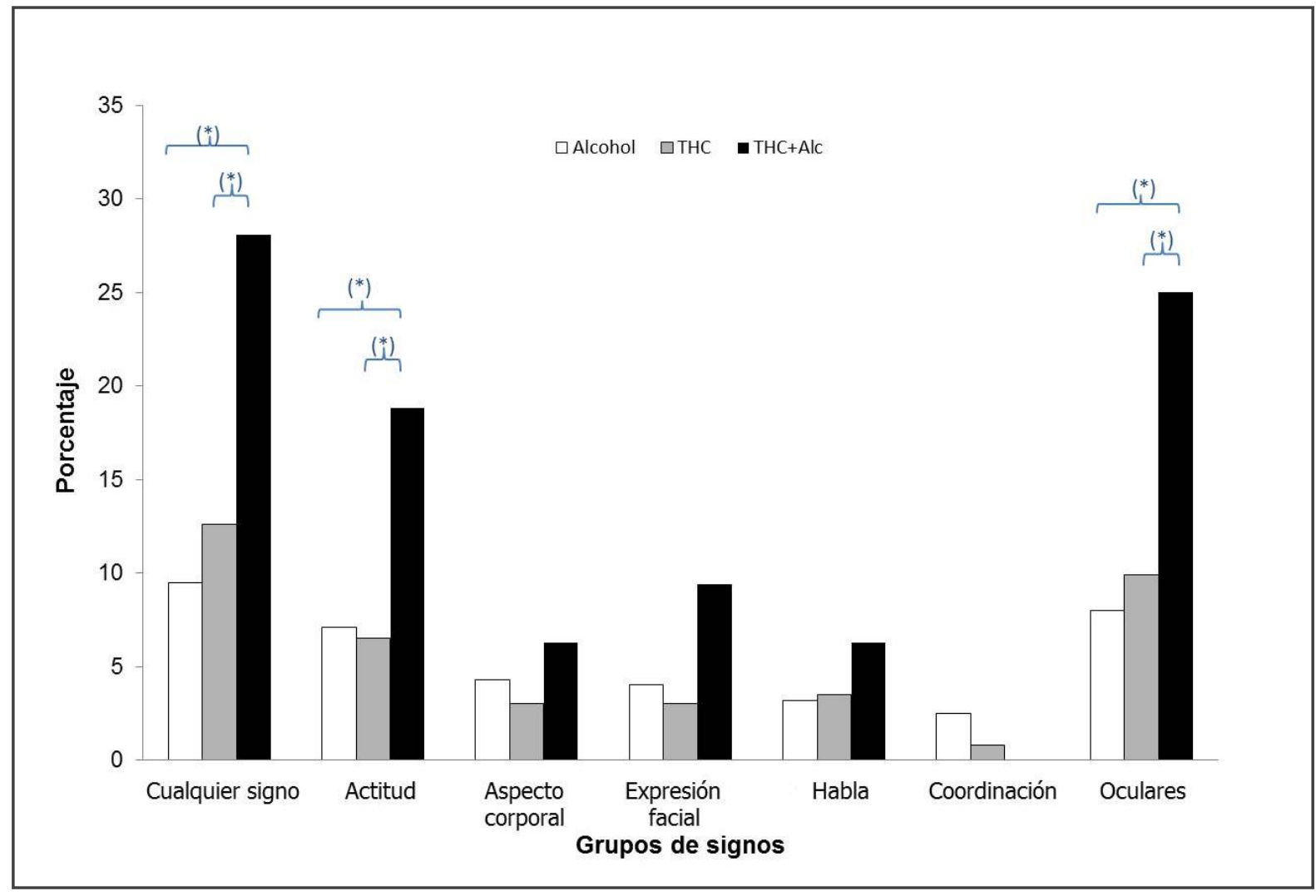

Figura 7 Prevalencia de conductores en los que se observaron signos (cualquier signo y agrupaciones de signos) según la sustancia detectada: Alcohol/THC/THC+Alcohol 
Tabla 6 Porcentaje de conductores en los que se ha observado algún signo de deterioro (cualquier signo, agrupados por tipos de signos y cada signo individual) en cada uno de los grupos del estudio.

\begin{tabular}{|c|c|c|c|c|}
\hline \multirow[t]{2}{*}{ Signo de deterioro observado } & \multicolumn{4}{|c|}{ Sustancia detectada } \\
\hline & $\begin{array}{l}\text { Ninguna } \\
(\mathrm{N}=2146)\end{array}$ & $\begin{array}{l}\text { THC } \\
(N=253)\end{array}$ & $\begin{array}{l}\text { Alcohol } \\
(N=201)\end{array}$ & $\begin{array}{l}\text { THC+alcohol } \\
(\mathrm{N}=32)\end{array}$ \\
\hline Cualquier signo (\%) & 1.0 & 12.6 & 9.5 & 28.1 \\
\hline Signos de actitud (\%) & 0.3 & 7.1 & 6.5 & 18.8 \\
\hline Nervioso (\%) & 0.2 & 4.3 & 2.0 & 9.4 \\
\hline Eufórico (\%) & 0.0 & 0.4 & 1.0 & 0.0 \\
\hline Provocativo (\%) & 0.0 & 0.8 & 0.0 & 3.1 \\
\hline Lloroso (\%) & 0.0 & 0.0 & 1.0 & 0.0 \\
\hline Adormilado (\%) & 0.0 & 2.0 & 3.0 & 9.4 \\
\hline Se rascal la cara (\%) & 0.0 & 0.0 & 0.0 & 3.1 \\
\hline No comprende (\%) & 0.0 & 0.0 & 0.5 & 3.1 \\
\hline Signos de aspecto corporal (\%) & 0.2 & 4.3 & 3.0 & 6.3 \\
\hline Temblor $(\%)$ & 0.0 & 1.6 & 0.0 & 0.0 \\
\hline Sudoración (\%) & 0.0 & 0.4 & 0.0 & 0.0 \\
\hline Inquietud $(\%)$ & 0.2 & 2.4 & 2.5 & 3.1 \\
\hline Respiración superficial (\%) & 0.0 & 0.0 & 1.0 & 3.1 \\
\hline Signos de expresión facial (\%) & 0.2 & 4.0 & 3.0 & 9.4 \\
\hline Parpadeo $(\%)$ & 0.2 & 2.4 & 2.5 & 6.3 \\
\hline Nariz roja (\%) & 0.0 & 0.0 & 1.0 & 0.0 \\
\hline Esnifa (\%) & 0.0 & 0.0 & 0.0 & 0.0 \\
\hline Traga saliva (\%) & 0.0 & 0.4 & 0.0 & 3.1 \\
\hline Olor a porro (\%) & 0.0 & 1.6 & 0.0 & 0.0 \\
\hline Signos del habla (\%) & 0.2 & 3.2 & 3.5 & 6.3 \\
\hline Locuacidad (\%) & 0.0 & 1.2 & 2.0 & 3.1 \\
\hline Habla dificultosa (\%) & 0.0 & 0.4 & 1.0 & 0.0 \\
\hline Tono bajo (\%) & 0.1 & 1.6 & 0.5 & 3.1 \\
\hline Signos de coordinación (\%) & 0.0 & 0.8 & 2.5 & 0.0 \\
\hline Tambaleante $(\%)$ & 0.0 & 0.0 & 1.0 & 0.0 \\
\hline Movimientos descoordinados (\%) & 0.0 & 0.8 & 1.0 & 0.0 \\
\hline Temblor general (\%) & 0.0 & 0.0 & 0.5 & 0.0 \\
\hline Temblor de piernas (\%) & 0.0 & 0.0 & 0.0 & 0.0 \\
\hline Signos oculares (\%) & 0.7 & 9.9 & 8.0 & 25.0 \\
\hline Conjuntiva enrojecida (\%) & 0.6 & 9.5 & 8.0 & 21.9 \\
\hline Movimiento ocular brusco (\%) & 0.0 & 0.8 & 0.5 & 3.1 \\
\hline Nistagmo amplio (\%) & 0.0 & 0.4 & 0.5 & 6.3 \\
\hline Nistagmo $45^{\circ}(\%)$ & 0.0 & 0.8 & 1.0 & 3.1 \\
\hline Nistagmo $30^{\circ}(\%)$ & 0.0 & 0.8 & 0.5 & 3.1 \\
\hline Pupila contraída (\%) & 0.0 & 0.8 & 0.0 & 0.0 \\
\hline Pupila dilatada (\%) & 0.0 & 0.4 & 0.5 & 6.3 \\
\hline Baja reacción pupilar (\%) & 0.0 & 1.6 & 0.5 & 3.1 \\
\hline
\end{tabular}


En la Tabla 7 se muestran los casos que, por comparación con los resultados obtenidos en la prueba estándar (análisis de laboratorio LC/MS/MS para THC y alcoholemia en aire espirado para el alcohol) resultaron ser, verdaderos positivos $(V P)$, verdaderos negativos (VN), falsos positivos (FP) y falsos negativos (FN). Estos resultados se muestran por separado, para cada uno de los grupos de conductores especificados en el estudio en los que se detectaron sustancias y, en la última columna, se muestran los resultados para todos los grupos conjuntamente, incluyendo el grupo en el que no se detectaron sustancias. Además se calcularon cinco índices para determinar la validez de la observación de signos como prueba diagnóstica: Los valores de sensibilidad (S), especificidad (E), valor predictivo del resultado positivo (VPP), valor predictivo del resultado negativo (VPN) y el valor global de la proporción de resultados válidos entre la totalidad de las pruebas efectuadas (VG), con su intervalo de confianza al 95\%, que se muestran también en la Tabla 7.

\section{Conclusiones}

- Existe una correlación positiva entre la concentración de THC en el fluido oral y la prevalencia de conductores en los que se observaron algunos de los signos de deterioro analizados: la presencia de signos de oculares se hace significativa a partir de $3.0 \mathrm{ng} / \mathrm{ml}$ de THC en el fluido oral y a partir de 25 $\mathrm{ng} / \mathrm{ml}$ son significativos los signos de comportamiento, expresión facial y del habla.

- Los análisis de regresión logística muestran una contribución independiente, pero no específica, del alcohol y del cannabis a los signos observados. Los efectos observados podrían describirse como la suma de los efectos del alcohol y del cannabis cuando éstos se consumen separadamente.

- La presencia de signos de deterioro alerta en gran medida del consumo de sustancias psicoactivas, sin embargo, la ausencia de ellos no garantiza la conducción libre del efecto de sustancias.

- Como test diagnóstico, la observación de los signos relacionados con la presencia de THC en el fluido oral, presenta una baja sensibilidad. Por lo 
tanto, la observación de los signos de deterioro tiene una utilidad muy limitada, para detectar la conducción bajo el efecto del cannabis en un control de carretera realizado de forma aleatoria.

- Desde el momento en que los avances científicos ofrecen mejores opciones, la observación de los signos de deterioro no parece ofrecer ninguna ventaja si se dispone de los medios técnicos y analíticos necesarios.

Tabla 7 Valor diagnóstico de la observación de signos de deterioro y del test de cribado en fluido oral para cada grupo de conductores y con los puntos de corte establecidos para cada sustancia*.

\begin{tabular}{|c|c|c|c|c|c|}
\hline \multirow[b]{2}{*}{ Test } & & \multirow[t]{2}{*}{$\begin{array}{l}\text { THC } \\
(\mathrm{N}=253)\end{array}$} & \multirow[t]{2}{*}{$\begin{array}{l}\text { Alcohol } \\
(\mathrm{N}=201)\end{array}$} & \multicolumn{2}{|l|}{$\begin{array}{l}\text { THC+alcohol } \\
(\mathrm{N}=32)\end{array}$} \\
\hline & & & & $\mathrm{THC}$ & Alcohol \\
\hline \multirow[t]{9}{*}{ Observación de signos } & VP & 26 & 13 & 4 & 4 \\
\hline & VN & 112 & 148 & 10 & 17 \\
\hline & FP & 6 & 6 & 5 & 5 \\
\hline & $\mathrm{FN}$ & 109 & 34 & 13 & 6 \\
\hline & Sensitivity & $19.3[12.2-26.3]$ & $27.7[13.8-41.5]$ & $23.5[0.4-46.6]$ & $40.0[4.6-75.4]$ \\
\hline & Specificity & 94.9 [90.5-99.3] & 96.1 [92.7-99.5] & 66.7 [39.5-93.9] & $77.3[57.5-97.1]$ \\
\hline & VPP & 81.3 [66.2-96.3] & 68.4 [44.9-92.0] & 44.4 [6.4-82.5] & $44.4[6.4-82.5]$ \\
\hline & VPN & 50.7 [43.9-57.5] & $81.3[75.4-87.3]$ & $43.5[21.0-65.9]$ & $73.9[53.8-94.0]$ \\
\hline & VG & 54.6 [48.2-60.9] & $80.1[74.3-85.9]$ & $43.75[25.0-62.5]$ & $65.6[47.6-83.6]$ \\
\hline \multirow[t]{9}{*}{ Cibado en fluido oral } & VP & 103 & & 13 & \\
\hline & VN & 110 & & 12 & \\
\hline & FP & 8 & & 3 & \\
\hline & $\mathrm{FN}$ & 32 & & 4 & \\
\hline & Sensitivity & $76.3[68.8-83.8]$ & & 76.5 [53.4-99.6] & \\
\hline & Specificity & 93.2 [88.3-98.2] & & $80.0[56.4-100.0]$ & \\
\hline & VPP & $92.8[87.5-98.1]$ & & $81.3[59.0-100.0]$ & \\
\hline & VPN & $77.5[70.2-84.7]$ & & 75.0 [50.7-99.3] & \\
\hline & VG & 84.2 [79.5-88.9] & & $78.1[62.2-94.0]$ & \\
\hline
\end{tabular}

*Puntos de corte establecidos: THC $>27 \mathrm{ng} / \mathrm{ml}$ en fluido oral y alcohol $>0.25 \mathrm{mg} / \mathrm{l}$ en aire espirado. En el análisis de referencia, para todos los grupos juntos fueron considerados casos positivos, aquellos casos que superaban el punto de corte en alguna de las sustancias. 


\subsection{Agresividad vial}

Se analizaron factores sociodemográficos, consumo de sustancias y adopción de conductas de riesgo relacionadas con el consumo de sustancias en la población general y en pacientes con trastorno por consumo de sustancias.

\subsubsection{Metodología}

Tanto en el capítulo 5, "Agresividad vial en la población general",[7] como en el capítulo 6, "Alcohol use, illicit drug use, and road rage",[8] la población objeto del estudio fueron los habitantes de Castilla y León de edad comprendida entre los 14 y los 70 años, según las cifras oficiales de población de 2007 (padrón municipal de 2008, http://www.ine.es). En el capítulo 7, "Road rage among drug dependent patiens" [9] la población objeto del estudio fueron, los pacientes drogodependientes que acudían al centro de tratamiento de Cruz Roja en Valladolid, en demanda de tratamiento. La población general de Castilla y León se utilizó, en este último trabajo, como población de referencia.

Para las tres publicaciones que constituyen los capítulos 5, 6 y 7 de este compendio [7-9] se utilizó la base de datos del estudio "El consumo de drogas en Castilla y León, 2008" [62]. Dicha base contiene 2500 registros de una muestra representativa de los habitantes de Castilla y León.

Además, para el estudio en drogodependientes se generó una nueva base de datos a partir de una muestra de 100 pacientes voluntarios. Estos pacientes fueron seleccionados aleatoriamente, entre aquellos que acudían semanalmente al control de drogas en orina (obligatorio para verificar la abstinencia y el correcto seguimiento del tratamiento) pero, con la condición de que estuvieran en sus primeros 12 meses de tratamiento.

Ante la falta de datos previos, el tamaño de la muestra se estableció en 100 entrevistas, bajo la hipótesis de un incremento adicional del $20 \%$ en la prevalencia de agresividad vial entre los pacientes drogodependientes, sobre la observada en la población general de Castilla y León $[7,9]$.

El cuestionario utilizado para "El consumo de drogas en Castilla y León, 2008" [62] se estructura en seis grandes apartados. 
$\checkmark$ Datos sociodemográficos y de filiación

$\checkmark$ Opiniones, actitudes y creencias de la población ante el tabaco, el alcohol y las drogas

$\checkmark$ Pautas del consumo de tabaco

$\checkmark$ Pautas del consumo de alcohol

$\checkmark$ Pautas del consumo de drogas

$\checkmark$ Agresividad en la conducción (Road Rage)

El apartado destinado a investigar la agresividad vial, tanto en la población general de Castilla y León, como en los drogodependientes, consta de una batería de 8 preguntas (Tabla 8) tomadas del trabajo de Smart y cols. [53]. En las cuatro primeras cuestiones se pregunta acerca de la experiencia de la agresividad vial como víctima y en los otros 4 ítems siguientes como posible agresor. En ambos casos (victimización y agresión) el orden de los ítems refleja una progresión en la severidad de la "expresión o manifestación violenta": i) manifestación de enfado y/o frustración, ii) intimidación y/o amenazas, iii) daños intencionales en el vehículo, iv) lesiones intencionales al conductor del vehículo o a alguno de sus ocupantes. Las ocho preguntas hacen referencia a la experiencia de la agresividad vial, en los 12 meses previos a la realización del cuestionario.

Para analizar los factores que influyen en la agresividad vial se consideran cada uno de los ítems por separado o, en otros casos, se agrupan las cuestiones definiendo así varias categorías en la experiencia de la agresividad vial. De esta forma se analiza: cualquier forma de experiencia de agresividad vial (víctima y/o agresor), víctima y agresor (Tabla 8). Se definen, además, dos niveles de agresión: leve (ha contestado afirmativamente al primero de los cuatro ítems de agresión y negativamente a los otros tres) y grave (ha contestado afirmativamente a alguno de los tres últimos ítems de agresor). 
Tabla 8 Agresividad vial: Cuestionario de Smart y cols. [53], y agrupación de los ítems.

Ítems víctima

1. ¿Le han gritado, insultado o hecho gestos obscenos desde otro vehículo, a usted o a alguno de los que viajaban con usted?

2. ¿Alguien de otro vehículo ha amenazado con agredirle a usted o a los que viajaban con usted, o le han amenazado con dañar el vehículo en el que usted viajaba?

3. ¿Alguien de otro vehículo ha dañado intencionadamente o ha intentado dañar el vehículo en el que usted viajaba?

4. ¿Alguien de otro vehículo le ha herido intencionadamente o ha intentado herirle a usted o a los que viajaban con usted?

Ítems agresor

5. ¿Ha gritado, insultado, o hecho gestos groseros a un conductor o pasajero de otro vehículo?

6. ¿Ha amenazado usted con agredir a un conductor o a un pasajero de otro vehículo, o ha amenazado con dañar su vehículo?

7. ¿Ha dañado o ha intentado dañar intencionadamente el vehículo de otro conductor?

8. ¿Ha lesionado o ha tratado de lesionar intencionadamente a otro conductor o a un pasajero de otro vehículo?

Agresividad vial: Sí (víctima y/o agresor) ${ }^{a}$

Víctimas ${ }^{\mathrm{b}}$

Agresores $^{c}$

Agresor «leve» ${ }^{d}$

Agresor «grave»

${ }^{\text {a }}$ Ha contestado afirmativamente a uno de los ocho ítems, o más.

${ }^{\mathrm{b}} \mathrm{Ha}$ contestado afirmativamente al menos a uno de los cuatro ítems de víctima.

${ }^{c}$ Ha contestado afirmativamente al menos a uno de los cuatro ítems de agresor.

${ }^{d} \mathrm{Ha}$ contestado afirmativamente al primero de los cuatro ítems de agresor y negativamente a los tres siguientes.

e Ha contestado afirmativamente a alguno de los tres últimos ítems de agresor.

\section{Otras variables incluidas}

Dependiendo de la publicación (Capítulo 4, 5 ó 6) y de los diferentes análisis realizados se utilizaron diferentes variables o alguna de las variables iniciales, pudo recodificarse con diferentes categorías en cada uno de los estudios. En general, además de los factores referentes a la agresividad vial anteriormente mencionados, las demás variables analizadas fueron:

Variables sociodemográficas. Sexo; edad (años); ser conductor o no; km conducidos al año; estado civil; tamaño de población; nivel de estudios; empleo. 
Variables relacionadas con el consumo de alcohol. Ser bebedor habitual o no; consumo de alcohol en unidades de bebida estándar por semana (UBEs/semana); nivel de consumo de alcohol (bajo moderado o alto); binge drinking $^{2}$ en los últimos 30 días; puntuación en el test CAGE $\geq 2$ (Tabla 9); conducir bajo la influencia del alcohol en los 30 días anteriores a la entrevista; montar como pasajero con un conductor bajo la influencia del alcohol.

Tabla 9 Test CAGE para la detección de problemas relacionados con el alcohol: preguntas originales (inglés), traducción al español y significado de cada punto (1 respuesta positiva $=1$ punto) [63].

C: Have you felt the need to Cut down drinking?

¿Ha sentido alguna vez que debe beber menos?

Busca intentar reducir el consumo de alcohol y revela los problemas individuales que surgen del abuso.

A: Have you ever felt Annoyed by criticism of drinking?

¿Le ha molestado que la gente lo critique por su forma de beber?

Encubre las consecuencias sociales negativas del consumo abusivo de alcohol y es el menos sensible y específico de los ítems.

G: Have you had Guilty feelings about drinking?

¿Alguna vez se ha sentido mal o culpable por su forma de beber?

Los sentimientos de culpa pertenecen a la dimensión psicopatológica del consumo excesivo de alcohol y son realmente frecuentes en estos trastornos.

E: Do you ever take a morning Eye opener (a drink first thing in the morning to steady your nerves or get rid of a hangover)?

¿Alguna vez ha necesitado beber por la mañana para calmar los nervios o eliminar molestias por haber bebido la noche anterior?

Es casi patognomónico de dependencia

Variables relacionadas con el consumo de drogas. Consumo en el año previo (cannabis, anfetaminas, cocaína, drogas de diseño, opiáceos, alucinógenos, inhalables); montar como pasajero con un conductor bajo la influencia del cannabis; conducir bajo la influencia del cannabis en los 12 meses previos a la entrevista; ser consumidor habitual de cannabis.

\footnotetext{
${ }^{2}$ Binge drinking: consumo de 5 bebidas alcohólicas o más en un corto intervalo de tiempo 
Interacción de variables relacionadas con el consumo. Bebedor habitual (si/no) x consumo de cannabis en el año previo (si/no); conducir bajo la influencia del alcohol (si/no) x conducir bajo la influencia del cannabis (si/no); montar como pasajero con un conductor bajo la influencia del alcohol (si/no) x montar como pasajero con un conductor bajo la influencia del cannabis (si/no).

Variables relacionadas con la dependencia. Sustancia por la que inició el tratamiento (Heroína+cocaína; heroína; cocaína; otras) y tratamiento (programa de mantenimiento con metadona; libre de drogas; psicoactivos).

\section{Análisis estadístico}

Para analizar la asociación entre variables categóricas se utilizó el test de la chi cuadrado de Pearson o el test exacto de Fischer cuando la frecuencia esperada era menor de 5. La comparación de medias se realizó mediante análisis de la varianza (ANOVA), el tests de la $T$ de Student o la $U$ de Mann-Whitney según número de grupos a comparar y condiciones de normalidad. La validez interna del test de agresividad vial (8 ítems) se analizó con el test alfa de Chronbach.

El análisis de regresión logística se utilizó para analizar la influencia, tanto de los factores sociodemográficos, como de los factores asociados al de consumo de sustancias, y a las actitudes derivadas del consumo relacionadas con la conducción. La influencia de todos estos factores se analizó separadamente para conductores y no conductores y para diferentes formas de experiencia de la agresividad vial (cualquier experiencia, como víctima, como agresor y, como víctima y agresor). Para aquellos que manifestaron haber sido agresores se analizó como influían los factores anteriores dependiendo del tipo de agresión (leve o grave, Tabla 7).

El análisis estadístico se realizó con el programa estadístico SPSS (v.15 y v.18). Para todos los tests estadísticos, el nivel de significación se estableció en $p \leq 0.05$. 


\subsubsection{Resultados}

En la tabla 10 se presentan, para la muestra de Castilla y León (total, conductores y no conductores), las principales variables sociodemográficas y relacionadas con consumo de sustancias.

La experiencia de la agresividad vial es frecuente entre los habitantes de Castilla y León (Capítulo 5). De los 2500 entrevistados, el 31.1\% refirió haber tenido alguna experiencia en el año previo a la realización de la encuesta (Figura 8). El $16.5 \%$ respondió afirmativamente a algún ítem de víctima y de agresor, el $11.0 \%$ fue solamente víctima y el $3.6 \%$ solamente agresor. Por lo tanto el $20.2 \%$ de los entrevistados incurrió en algún comportamiento de agresión vial: el 17.6\% perpetraron agresiones leves (insultos o gestos obscenos) y el $2.6 \%$, agresiones más graves (amenazas, tentativas de daños a personas o vehículos e incluso, daños consumados).

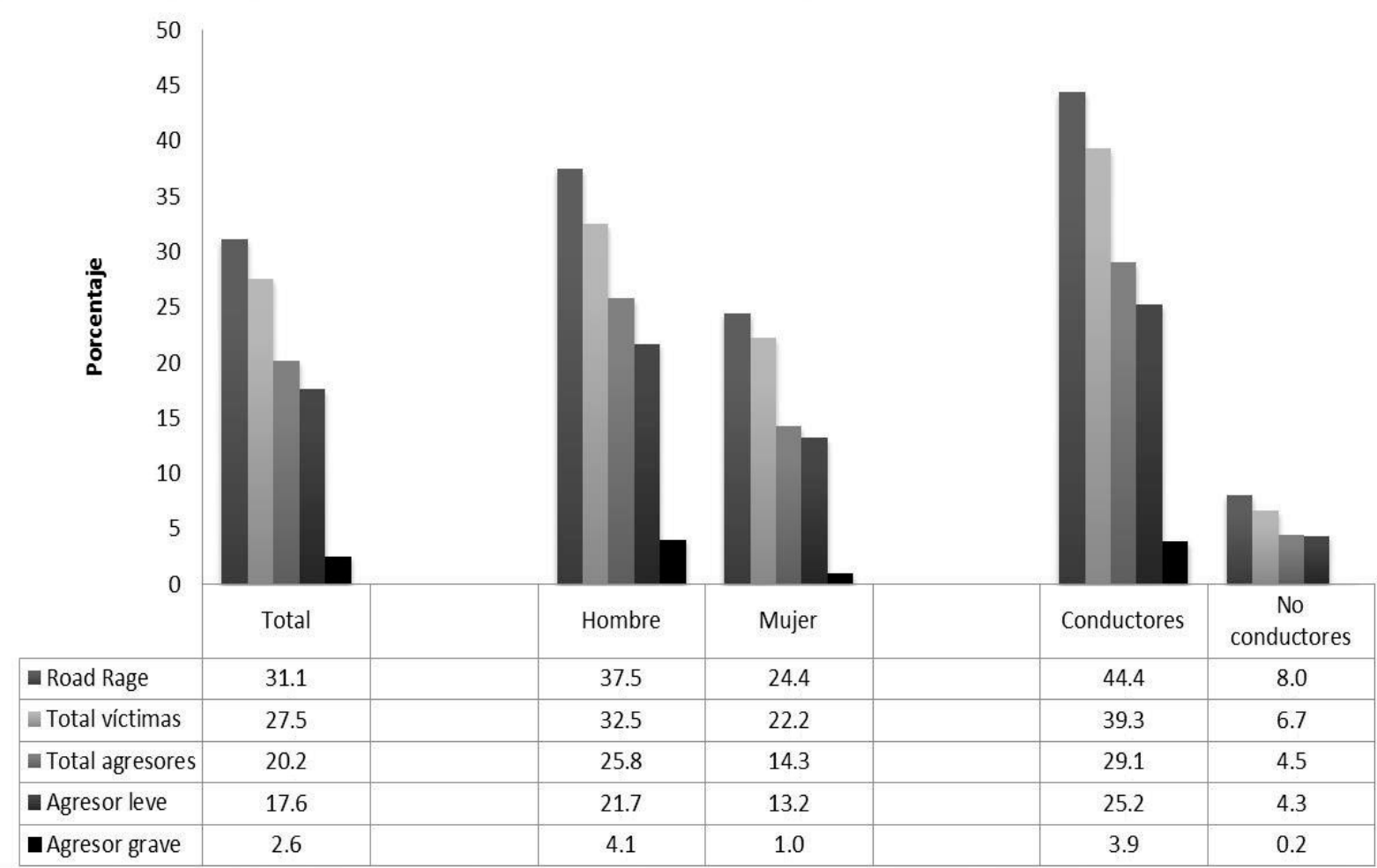

Figura 8 Agresividad vial (Road Rage) en la población general de Castilla y León, 2008. 
Tabla 10 Variables sociodemográficas y relacionadas con el consumo de sustancias analizadas en la población de Castilla y León. Total, conductores y no conductores.

\begin{tabular}{|c|c|c|c|}
\hline Variables & $\begin{array}{l}\text { Total } \\
\mathbf{N}=\mathbf{2 5 0 0}\end{array}$ & $\begin{array}{l}\text { Conductores } \\
\mathrm{N}=1591\end{array}$ & $\begin{array}{l}\text { No } \\
\text { Conductores } \\
\mathrm{N}=909\end{array}$ \\
\hline \multicolumn{4}{|l|}{ Sociodemográficas } \\
\hline \multicolumn{4}{|l|}{$\operatorname{Sexo}(n ; \%)$} \\
\hline Hombre & $1276 ; 51.0$ & $983 ; 61.8$ & $293 ; 32.2^{* * *}$ \\
\hline Mujer & $1224 ; 49.0$ & $608 ; 39.2$ & $616 ; 67.8$ \\
\hline Edad (media $\pm D E$ ) & $41.4 \pm 15.2$ & $41.2 \pm 13$ & $41.9 \pm 18.5$ \\
\hline \multicolumn{4}{|l|}{ Estado civil $(n ; \%)^{a}$} \\
\hline Casado/con pareja & $1297 ; 51.9$ & $882 ; 55.4$ & $415 ; 45.7^{* * *}$ \\
\hline Otros & $1203 ; 48.1$ & $709 ; 44.6$ & $494 ; 54.3$ \\
\hline \multicolumn{4}{|l|}{ Nivel de estudios $(n ; \%)^{a}$} \\
\hline Básicos/sin estudios & $1945 ; 77.8$ & $1142 ; 71.8$ & $803 ; 88.3^{* * *}$ \\
\hline Secundaria o superior & $555 ; 22.2$ & $449 ; 28.2$ & $106 ; 11.7$ \\
\hline \multicolumn{4}{|l|}{ Tamaño de la población $(\mathrm{n} ; \%)^{\mathrm{a}}$} \\
\hline$\geq 10000$ habitantes & $1432 ; 57.3$ & $887 ; 55.8$ & $545 ; 60.0^{*}$ \\
\hline$<$ than 10000 habitantes & $1068 ; 42.7$ & $704 ; 44.2$ & $364 ; 40.0$ \\
\hline Miles de Km/año conducidos (media $\pm \mathrm{DE}$ ) & & $17.2 \pm 26.1$ & \\
\hline \multicolumn{4}{|l|}{ Consumo de alcohol } \\
\hline Bebedor habitual ( $n ; \%)$ & $889 ; 35.6$ & $639 ; 40.2$ & $250 ; 27.5^{* * *}$ \\
\hline UBEs/semana (mean \pm SD) & $4.5 \pm 9.7$ & $5.2 \pm 10.0$ & $3.2 \pm 8.9^{* * *}$ \\
\hline \multicolumn{4}{|l|}{ Nivel de consumo de alcohol $(n ; \%)$} \\
\hline Bajo: hombres $\leq 21 \mathrm{UBE} / \mathrm{sem}$; mujeres $\leq 14 \mathrm{UBE} / \mathrm{sem}$. & $713 ; 28.5$ & $506 ; 31.8$ & $207 ; 22.8^{* * *}$ \\
\hline Moderado: hombres 22-50 UBE/wk.; mujeres 15-35 UBE/sem & $154 ; 6.2$ & $121 ; 7.6$ & $33 ; 3.6$ \\
\hline Alto: hombres > 50 UBE/sem.; mujeres > 35 UBE/sem. & $22 ; 0.9$ & $12 ; 0.8$ & $10 ; 1.1$ \\
\hline Cinco o más bebidas en una ocasión (Binge drinking) ( $n$; \%) & $439 ; 17.6$ & $299 ; 18.8$ & $140 ; 15.4$ \\
\hline CAGE 2 o más puntos $(n ; \%)$ & $153 ; 6.1$ & $99 ; 6.2$ & $54 ; 5.9$ \\
\hline Condujo bajo la influencia del alcohol en año previo ( $n$; \%) & $108 ; 4.3$ & $108 ; 6.8$ & $0 ; 0.0$ \\
\hline Pasajero con conductor bajo la influencia del alcohol ( $\mathrm{n} ; \%)$ & $144 ; 5.8$ & $94 ; 5.9$ & $50 ; 5.5$ \\
\hline \multicolumn{4}{|l|}{ Consumo de drogas (año previo) } \\
\hline Cannabis $(n ; \%)$ & $192 ; 7.7$ & $142 ; 8.9$ & $50 ; 5.5$ \\
\hline Anfetaminas $(n ; \%)^{b}$ & $21 ; 0.8$ & $16 ; 1.0$ & $5 ; 0.6$ \\
\hline Cocaína $(n ; \%)^{b}$ & $47 ; 1.9$ & $43 ; 2.7$ & $4 ; 0.4$ \\
\hline Drogas de diseño / éxtasis $(n ; \%)^{b}$ & $22 ; 0.9$ & $19 ; 1.2$ & $3 ; 0.3$ \\
\hline Tranquilizantes $(\mathrm{n} ; \%)^{\mathrm{b}}$ & $13 ; 0.5$ & $10 ; 0.6$ & $3 ; 0.3$ \\
\hline Opiáceos $(n ; \%)^{b}$ & $10 ; 0.4$ & $10 ; 0.6$ & $0 ; 0.0$ \\
\hline Alucinógenos $(n ; \%)^{b}$ & $12 ; 0.5$ & $11 ; 0.7$ & $1 ; 0.1$ \\
\hline Inhalables $(n ; \%)^{b}$ & $2 ; 0.1$ & $1 ; 0.1$ & $1 ; 0.1$ \\
\hline Conductor bajo influencia del cannabis ( $n ; \%)$ & $35 ; 1.4$ & $35 ; 2.2$ & $0 ; 0.0$ \\
\hline Pasajero con conductor bajo la influencia del cannabis ( $n$; \%) & $63 ; 2.5$ & $48 ; 3.0$ & $15 ; 1.7$ \\
\hline \multicolumn{4}{|l|}{ Alcohol y cannabis } \\
\hline Bebedor habitual + cannabis en año previo $(n ; \%)$ & $135 ; 5.4$ & $101 ; 6.3$ & $34 ; 3.7$ \\
\hline $\begin{array}{l}\text { Conductor bajo influencia del alcohol + cannabis en año previo } \\
(\mathrm{n} ; \%)\end{array}$ & $12 ; 0.5$ & $12 ; 0.8$ & $0 ; 0.0$ \\
\hline $\begin{array}{l}\text { Pasajero con conductor bajo la influencia del alcohol + cannabis } \\
\text { en año previo }(n ; \%)\end{array}$ & $25 ; 1.0$ & $19 ; 1.2$ & $6 ; 0.7$ \\
\hline
\end{tabular}


Como se observa en la figura 9, la prevalencia de agresividad vial es mucho mayor entre los pacientes drogodependientes analizados que en la población general (Capítulo 7). El $63.0 \%$ de los pacientes contestó afirmativamente a alguna de las ocho preguntas del test (el $91.1 \%$ de los conductores y el $40.0 \%$ de los no conductores). Además, el porcentaje de los agresores alcanzaba el $52.0 \%$ (el $77.8 \%$ de los conductores y el $30.9 \%$ de los no conductores). El $18.0 \%$ refirieron agresiones graves (el $31.0 \%$ de los conductores y el $7.3 \%$ de los no conductores). En la figura 9 se añaden, además, los resultados obtenidos para una subpoblación extraída de la base de datos de la población general: los 217 entrevistados que refirieron haber consumido drogas en el año previo a la realización de la entrevista.

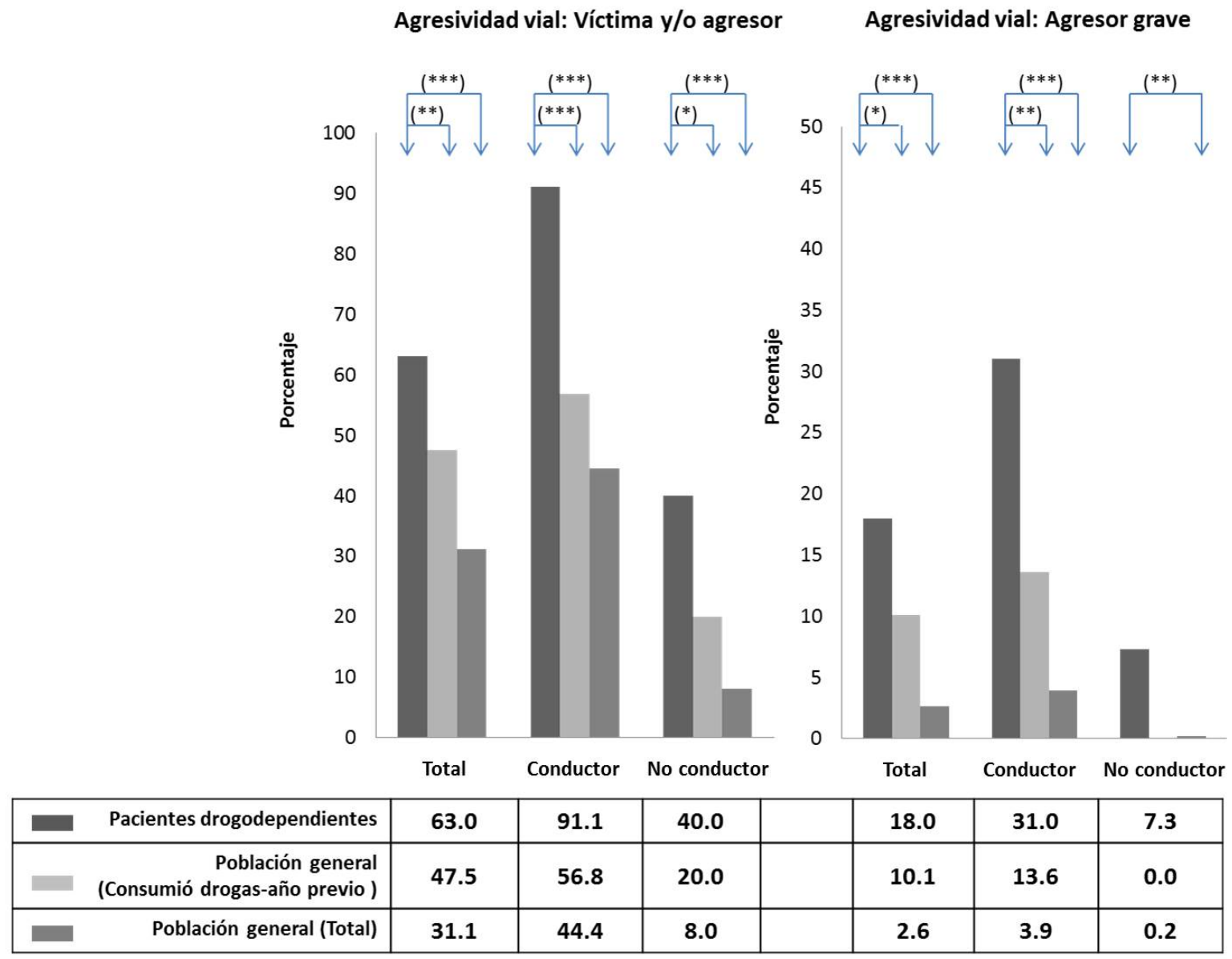

Figura 9 Agresividad vial y agresiones graves: prevalencia en pacientes drogodependientes, en la población general y en los que refirieron consumo de drogas durante el año previo. Diferencias significativas en el test de la chi cuadrado de Pearson señaladas con asteriscos (*** $\mathrm{p}<0.0001 ; * * \mathrm{p}<0.001 ; * \mathrm{p}<0.05)$. 
En la población general no conductora, la edad es el único factor sociodemográfico que influye sobre la probabilidad de verse involucrado en situaciones de agresividad vial (OR [IC 95\%] $=0.969$ [0.956-0.983], sin controlar los factores de consumo de sustancias).

En el caso de los conductores, con la edad también disminuye la probabilidad de verse involucrado en episodios de agresividad vial (0.975 [0.967-0.983]), pero además, influyen otros factores sociodemográficos: Es mayor para los hombres (1.287 [1.034-1.602]), entre las personas con estudios universitarios (1.408 [1.121-1.773]), si la población es de 10000 habitantes o mayor (1.250 [1.0171.535]) y también aumenta con los miles de kilómetros conducidos a la semana $(1.520[1.202-1.921])$.

Cuando se incluyen las variables relacionadas con el consumo de sustancias en el análisis (Capítulo 6) se observa que conducir bajo la influencia del alcohol o del cannabis son variables asociadas a conductores con un mayor riesgo de ser agresores (solo agresores). Conducir bajo la influencia del alcohol y/o del cannabis y ser un bebedor problemático (puntuación CAGE $\geq 2$ ), son variables asociadas a conductores con un mayor riesgo de experiencias de agresividad vial como víctima y agresor (ambas, Tabla 11) así como a un mayor riesgo de involucrarse, como perpetrador, en agresiones graves (Tabla 12).

Entre los no conductores, el riesgo de ser solo agresor o víctima y agresor (ambos) es mayor entre los que montaron como pasajeros con un conductor bajo los efectos del alcohol y/o del cannabis. En los no conductores, el mayor riesgo de ser agresor se refiere, al contrario de lo que ocurre con los conductores, a agresiones leves (Tablas 11 y 12). 


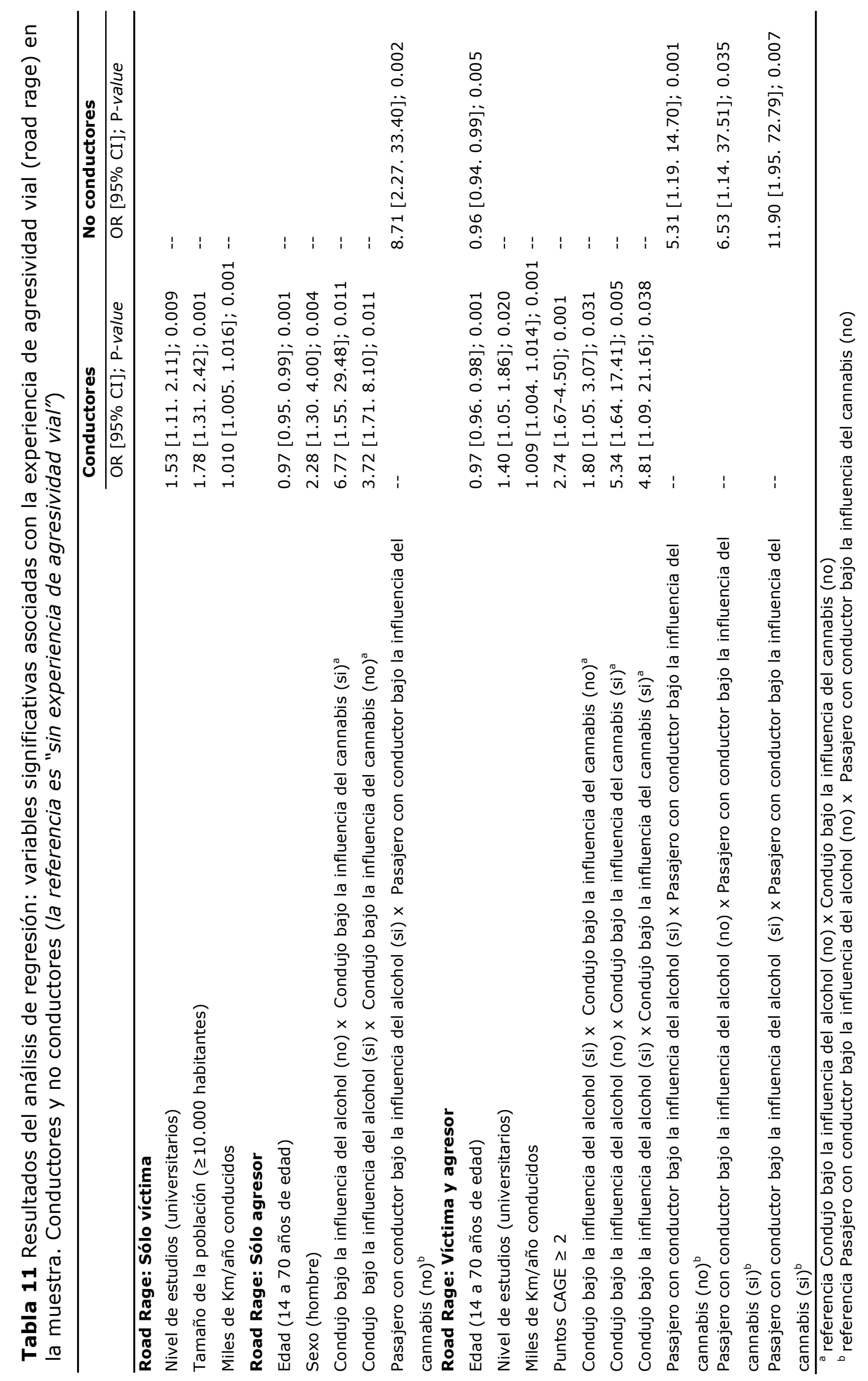




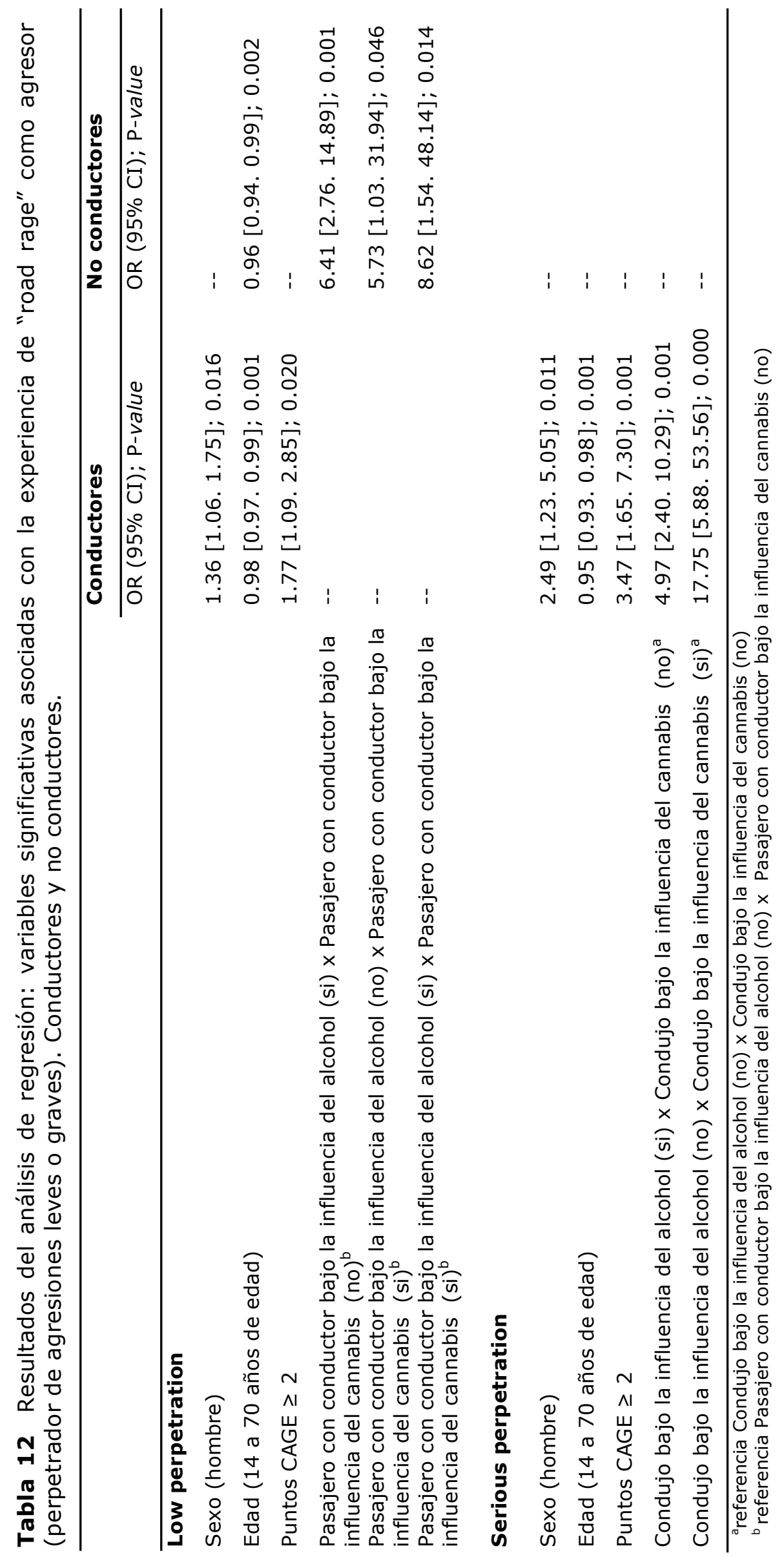




\section{Conclusiones}

- La agresividad vial es frecuente en la población general de Castilla y León (31.1\%). Es significativamente más elevada entre los que habían consumido drogas el año previo (47.5\%) y más elevada aún entre los pacientes drogodependientes (63.0\%).

- Aunque la experiencia de agresividad vial es más frecuente entre los conductores (el $44.0 \%$ en la población general y el $91.1 \%$ en drogodependientes) también afecta a los no conductores $(8.0 \%$ y $40.0 \%$ respectivamente).

- Conducir habiendo consumido alcohol o cannabis (una sola sustancia o ambas) o ser un bebedor problemático (puntos $C A G E \geq 2$ ), está asociado a un mayor riesgo de ser un perpetrador de agresiones viales graves y a una mayor prevalencia en la experiencia de agresividad vial, tanto en el papel de víctima como de agresor.

- Entre los no conductores, montar como pasajero con un conductor que ha consumido alcohol o cannabis (una sola sustancia o ambas) incrementa la probabilidad agresividad vial siendo víctima y agresor y de ser agresor de agresiones leves (insultos y gestos). 


\section{Conclusiones por objetivos}

\subsection{Prevalencia de conductores españoles que conducen con la presencia de sustancias psicoactivas evolución (2008-2013).}

- Conducir después del consumo de sustancias psicoactivas es un hecho frecuente en España, alcanzando el 17\% de los conductores españoles.

- Casi un $11 \%$ de conductores conducen tras haber consumido otras sustancias psicoactivas distintas al alcohol.

- Cannabis y cocaína explican la mayor parte de los casos positivos a drogas.

- En lo que respecta a las drogas de comercio ilegal, la prevalencia disminuye a medida que aumenta la edad de los conductores, es más frecuente en varones, en vías urbanas y durante la madrugada, tanto en fin de semana como en días laborales.

- En 2013, se observa un descenso en la prevalencia de casos positivos a sustancias $(9.34 \%)$ en los conductores españoles, respecto del estudio llevado a cabo en el año 2008 (14.05\%).

En consecuencia, aunque la prevalencia de conductores con presencia de sustancias sigue siendo alta en las carreteras españolas, la realización de controles aleatorios de carretera, con análisis de la presencia de drogas en saliva además de los controles de alcohol en aire espirado, parece ser la causa disuasoria más probable para explicar el descenso en la prevalencia. 


\subsection{Comprensibilidad del pictograma español sobre medicamentos y conducción impreso en el envase de algunos medicamentos, y su aceptación por parte de los usuarios de medicamentos.}

- La mayor parte de los entrevistados (el 93.9\%) sabía que algunos medicamentos podían afectar a su capacidad de conducir.

- El pictograma español sobre medicamentos y conducción es comprendido por la mayoría de los entrevistados. El $85.7 \%$ (el $90.5 \%$ de los conductores y el $67.4 \%$ de los no conductores) relacionaron correctamente el símbolo con los posibles efectos del medicamento sobre la conducción.

- Con una puntuación global media de 7.98 1.58 puntos, los usuarios del Sistema Nacional de Salud consideraron este pictograma "útil", "informativo", "comprensible" y "sencillo".

- Su presencia en el envase de los medicamentos podría ejercer una influencia en la actitud de los conductores: el $83.9 \%$ reduciría su frecuencia de conducción si le prescribieran un medicamento con pictograma.

Por lo tanto, este pictograma puede considerarse una herramienta para mejorar los procedimientos de prescripción y dispensación de medicamentos que afecten a la capacidad de conducir y también, como un instrumento para concienciar a los pacientes acerca del papel que los medicamentos pueden jugar en la seguridad vial.

\subsection{Relación entre signos de deterioro en conductores y las concentraciones de THC en el fluido oral y alcohol en aire espirado.}

- Existe una correlación positiva entre la concentración de THC en el fluido oral y la prevalencia de conductores en los que se observaron algunos de los signos de deterioro analizados: la presencia de signos de oculares se hace significativa a partir de $3.0 \mathrm{ng} / \mathrm{ml}$ de $\mathrm{THC}$ en el fluido oral y a partir de 25 
$\mathrm{ng} / \mathrm{ml}$ son significativos los signos de comportamiento, expresión facial y del habla.

- Los análisis de regresión logística muestran una contribución independiente, pero no específica, del alcohol y del cannabis a los signos observados. Los efectos observados podrían describirse como la suma de los efectos del alcohol y del cannabis cuando éstos se consumen separadamente.

- Como test diagnóstico, la observación de los signos relacionados con la presencia de THC en el fluido oral, presenta una baja sensibilidad.

Por lo tanto, la observación de los signos de deterioro tiene una utilidad muy limitada, para detectar la presencia de cannabis en el organismo del conductor, en un control de carretera realizado de forma aleatoria. Desde el momento en que los avances científicos ofrecen mejores opciones, la observación de los signos de deterioro no parece ofrecer ninguna ventaja si se dispone de los medios técnicos y analíticos necesarios.

\subsection{Relación entre la agresividad vial y la adopción de ciertas conductas de riesgo relacionadas con el consumo de sustancias y la conducción, en la población general y en pacientes con trastorno por consumo de sustancias.}

- La agresividad vial es frecuente en la población general de Castilla y León $(31.1 \%)$. Es significativamente más elevada entre los que habían consumido drogas el año previo (47.5\%) y más elevada aún entre los pacientes drogodependientes (63.0\%).

- Aunque la experiencia de agresividad vial es más frecuente entre los conductores (el $44.0 \%$ en la población general y el $91.1 \%$ en drogodependientes) también afecta a los no conductores (8.0\% y $40.0 \%$ respectivamente). 
- Conducir habiendo consumido alcohol o cannabis (una sola sustancia o ambas) o ser un bebedor problemático (puntos CAGE $\geq 2$ ), está asociado a un mayor riesgo de ser un perpetrador de agresiones viales graves y a una mayor prevalencia en la experiencia de agresividad vial, tanto en el papel de víctima como de agresor.

- Entre los no conductores, montar como pasajero con un conductor que ha consumido alcohol o cannabis (una sola sustancia o ambas) incrementa la probabilidad agresividad vial siendo víctima y agresor y de ser agresor de agresiones leves (insultos y gestos).

La agresividad vial es un fenómeno complejo que puede depender de múltiples factores. Sin embargo, la elevada prevalencia de agresividad vial entre los pacientes drogodependientes, así como su relación con el consumo problemático de alcohol y con el ejercicio de la conducción después de consumir alcohol y cannabis, sugieren la necesidad de desarrollar intervenciones apropiadas, dirigidas a reducir ciertas prácticas de riesgo en la carretera. 


\section{Referencias}

1. Organización Mundial de la Salud. Informe sobre la situación mundial de la seguridad vial. OMS. http://www.who.int/violence_injury_prevention/road_safety_status/report /web_version_es.pdf?ua=1 (acceso 20/01/2015)

2. Verstraete, A.G., Legrand, S.A, 2014. Drug use, Impaired Driving and Traffic Accidents. European Monitoring Centre for Drugs and Drug Addiction (EMCDDA), Lisbon. (acceso 18/05/2014)

3. Peden, M. Scurfield, R. Sleet, D. Mohan, D. Hyder, A.A. Jarawan, E. Mathers, C., 2004. World report on road traffic injury prevention. World Health Organization (WHO), Geneva. http://whqlibdoc.who.int/publications/2004/9241562609.pdf. (acceso 20/01/15)

4. Gomez-Talegon, T., Fierro, I., Gonzalez-Luque, J.C., Colas, M., Lopez-Rivadulla, M., Javier Alvarez, F., 2012. Prevalence of psychoactive substances, alcohol, illicit drugs, and medicines, in Spanish drivers: a roadside study. Forensic Sci Int. 223, 106-113.

5. Fierro, I., Gonzalez-Luque, J.C., Alvarez, F.J., 2014. The relationship between observed signs of impairment and THC concentration in oral fluid. Drug Alcohol Depend. 144, 231-238.

6. Alvarez, F.J., Fierro, I., 2014. Informe 1. Alcohol drogas y medicamentos en conductores de vehículos en España. Contratación de un servicio para la realización de un estudio de sustancias psicoactivas en conductores de vehículos en España. (No Exp.: 0100DGT23059).

7. Fierro, I., Gomez-Talegon, T., Alvarez, F.J., 2010. Road-rage in the general population. Gac Sanit. 24, 423-427.

8. Fierro, I., Morales, C., Alvarez, F.J., 2011. Alcohol use, illicit drug use, and road rage. J Stud Alcohol Drugs. 72, 185-193.

9. Benavidez, D.C., Flores, A.M., Fierro, I., Alvarez, F.J., 2013. Road rage among drug dependent patients. Accid Anal Prev. 50, 848-853.

10. Fierro, I., Gomez-Talegon, T., Alvarez, F.J., 2013. The Spanish pictogram on medicines and driving: The population's comprehension of and attitudes towards its use on medication packaging. Accid Anal Prev. 50, 1056-1061.

11. Walsh, J.M., de Gier, J.J., Christopherson, A.S., Verstraete, A.G., 2004. Drugs and driving. Traffic Inj Prev. 5, 241-253. 
12. Ramaekers, J.G., Kuypers, K.P., Bosker, W.M., Brookhuis, K.A., Veldstra, J.A., Simons, R., Martens, M., Hjalmdahl, M., Forsman, A., Knoche, A., 2012. Effects of stimulant drugs on actual and simulated driving: perspectives from four experimental studies conducted as part of the DRUID research consortium. Psychopharmacology (Berl).

13. Reeve, V.C., Grant, J.D., Robertson, W., Gillespie, H.K., Hollister, L.E., 1983. Plasma concentrations of $\delta$-9-tetrahydrocannabinol and impaired motor function. Drug Alcohol Depend. 11, 167-175.

14. Sewell, R.A., Poling, J., Sofuoglu, M., 2009. The effect of cannabis compared with alcohol on driving. Am J Addict. 18, 185-193.

15. Laumon, B., Gadegbeku, B., Martin, J.-L., \& Biecheler, M.-B., the SAM Group., 2005. Cannabis intoxication and fatal road crashes in France: Population based case-control study. BMJ. 331, 1371-1376.

16. Chipman, M.L., Macdonald, S., Mann, R.E., 2003. Being "at fault" in traffic crashes: Does alcohol, cannabis, cocaine, or polydrug abuse make a difference? Inj Prev. 9, 343-348.

17. Macdonald, S., Anglin-Bodrug, K., Mann, R. E., Erickson, P., Hathaway, A., Chipman, M., Rylett, M., 2003. Injury risk associated with cannabis and cocaine use. Drug Alcohol Depen. 72, 99-115.

18. Hels, T., Lyckegaard, A., Simonsen, K.W., Steentoft, A., Bernhoft, I.M., 2013. Risk of severe driver injury by driving with psychoactive substances. Accid Anal Prev. 59, 346-356.

19. Ahlm, K., Bjornstig, U., Ostrom, M., 2009. Alcohol and drugs in fatally and non-fatally injured motor vehicle drivers in northern Sweden. Accid Anal Prev. 41, 129-136.

20. Asbridge, M., Mann, R., Cusimano, M.D., Trayling, C., Roerecke, M., Tallon, J.M., Whipp, A., Rehm, J., 2014. Cannabis and traffic collision risk: findings from a casecrossover study of injured drivers presenting to emergency departments. Int J Public Health. 59, 395-404.

21. Albrecht, M., 2008. The "Driving under the Influence of Drugs, Alcohol and Medicines" (DRUID) project of the European Commission. Dtsch Med Wochenschr. 133 Suppl 2, S45-6.

22. Choo, R.E., Huestis, M.A., 2004. Oral fluid as a diagnostic tool. Clin Chem Lab Med. 42, 1273-1287. 
23. Gjerde, H., Verstraete, A.G., 2011. Estimating equivalent cutoff thresholds for drugs in blood and oral fluid using prevalence regression: a study of tetrahydrocannabinol and amphetamine. Forensic Sci Int. 212, e26-30.

24. Verstraete, A.G., 2005. Oral fluid testing for driving under the influence of drugs: history, recent progress and remaining challenges. Forensic Sci Int. 150, 143-150.

25. Pil, K., Verstraete, A., 2008. Current developments in drug testing in oral fluid. Ther Drug Monit. 30, 196-202.

26. BOE número 63 de 14/03/1990, Real Decreto Legislativo 339/1990, de 2 de marzo, por el que se aprueba el texto articulado de la Ley sobre Tráfico, Circulación de Vehículos a Motor y Seguridad Vial. Título II, Capítulo primero.

27. BOE número 288 de 1/12/2007, Ley Orgánica 15/2007, de 30 de noviembre, Capítulo IV, Título XVII, artículos 379.2 y 383, 2007, p. 49505.

28. BOE número 85 de 08/04/2014, Ley 6/2014, de 7 de abril, por la que se modifica el texto articulado de la Ley sobre Tráfico, Circulación de Vehículos a Motor y Seguridad Vial, aprobado por el Real Decreto Legislativo 339/1990, de 2 de marzo.

29. BOE número 152 de 23/06/2010, Ley Orgánica 5/2010, de 22 de junio, por la que se modifica la Ley Orgánica 10/1995, de 23 de noviembre, del Código Penal.

30. Engeland, A., Skurtveit, S., Morland, J., 2007. Risk of road traffic accidents associated with the prescription of drugs: a registry-based cohort study. Ann Epidemiol. 17: 597-602.

31. Orriols, L., Salmi, L.R., Philip, P., Moore, N., Delorme, B., Castot, A., et al., 2009. The impact of medicinal drugs on traffic safety: a systematic review of epidemiological studies. Pharmacoepidemiol Drug Saf. 18: 647-58.

32. Orriols, L., Delorme, B., Gadegbeku, B., Tricotel, A., Contrand, B., Laumon, B., et al., 2010. Prescription medicines and the risk of road traffic crashes: a French registrybased study. PLoS Med. 7: e1000366.

33. Gómez-Talegón, T., Fierro, I., Del Río, M.C., Álvarez, F.J., Establishment of framework for classification/categorisation and labelling of medicinal drugs and driving. Deliverable 4.3.1, 2011. DRUID (Driving under the Influence of Drugs, Alcohol and Medicines). 6th Framework programme. Disponible en; http://www.druid-project.eu/ (Acceso 19/06/2014)

34. Ravera, S., Monteiro SP, de Gier, J.J., van der Linden, T., Gómez-Talegón, T., Alvarez, F.J., the DRUID Project WP4 Partners., 2012. A European approach to categorising medicines for fitness to drive: outcomes of the DRUID project. $\mathrm{Br} \mathrm{J}$ of Clin Pharmacol. 74: 920-31. 
35. Ministerio de Sanidad y Consumo. REAL DECRETO 1345/2007, de 11 de octubre, por el que se regula el procedimiento de autorización, registro y condiciones de dispensación de los medicamentos de uso humano fabricados industrialmente. BOE 2007; 267: 45652-45698.

36. Ministère de la Santé et des Solidarités. Arrêté du 18 Juillet 2005 pris pour I'application de I'article R.5121-139 du code de la santé publique et relative à l'opposition d'un pictogramme sur le conditionnement extérieur de certain médicaments et produits. JO 2005.

37. European Council Directive 83/570/EEC of 26 October 1983 on the approximation of provisions laid down by law, regulation or administrative action relating to proprietary medicinal products, 1983. (Acceso 18/07/2014) Disponible en; http://eur-lex.europa.eu/LexUriServ/LexUriServ.do?uri=CELEX:31983L0570:EN:HTML

38. Bernardini, C., Ambrog,i V., Perioli, L.C., Tiralti, M.C., Fardella, G., 2000. Comprehensibility of the package leaflets of all medicinal products for human use: a questionnaire survey about the use of symbols and pictograms. Pharmacol Res. 41: 679-88.

39. Nathan JP, Zerilli T, Cicero LA, Rosenberg JM. Patients' use and perception of medication information leaflets. Ann Pharmacother. 2007; 41: 777-82.

40. Barrio-Cantalejo IM, Simon-Lorda P, March Cerda JC, Prieto Rodriguez MA. Grammatical readability of the package leaflets of the medicinal products most widely consumed and generating the highest expense in Spain during 2005]. Rev Esp Salud Publica. 2008; 82: 559-66.

41. Agencia Española de Medicamentos y Productos sanitarios (AEMPS). El pictograma; 2010; Disponible en; http://www.aemps.gob.es/ industria/ etiquetado/conduccion/ pictograma.htm (Acceso 20/01/2015)

42. NHTSA, 2001. Development of a Standardized Field Sobriety Test (SFST). Training Management System. DOT HS 809 400. http://www.nhtsa.gov/ people/ injury/alcohol/ sfst/introduction.htm (acceso 20/01/15).

43. Vindenes, V., Boix, F., Koksaeter, P., Strand, M.C., Bachs, L., Morland, J., Gjerde, H., 2014. Drugged driving arrests in Norway before and after the implementation of per se law. Forensic Sci Int. 245C, 171-177. 
44. Grotenhermen, F., Leson, G., Berghaus, G., Drummer, O.H., Kruger, H.P., Longo, M., Moskowitz, H., Perrine, B., Ramaekers, J.G., Smiley, A., Tunbridge, R., 2007. Developing limits for driving under cannabis. Addiction. 102, 1910-1917.

45. Burns, M., Moskowitz, H., 1977. Psychophysical Test for DWI Arrest. U.S. Department of Transportation, National HighwayTraffic safety Administration, Washington, D.C.

46. Tharp, V., Burns, M., and Moskowitz, H., 1981. Development and Field Test of Psychophysical Tests for DWI Arrest. U.S. Department of Transportation, National Highway Traffic Safety Administration, DOT-HS-805-864, Washington, D.C.

47. NHTSA, 2001. Development of a Standardized Field Sobriety Test (SFST). Training Management System. DOT HS 809 400. http: //www.nhtsa.gov/ people/ injury / alcohol / sfst/ introduction.htm (acceso 12/02/15).

48. Dupont, R.L., Voas, R.B., Walsh, J.M., Shea, C., Talpins, S.K., Neil, M.M., 2012. The need for drugged driving per se laws: a commentary. Traffic Inj Prev. 13, 31-42.

49. Mann, R.E., Zhao, J., Stoduto, G., Adlaf, E.M., Smart, R.G., \& Donovan, J.E., 2007. Road rage and collision involvement. Am J Health Behav. 31, 384-391.

50. Smart, R.G., Mann, R. E., 2002. Deaths injuries from road rage: cases in Canadian newspapers. CMAJ. 167, 761-762.

51. Smart, R.G., Mann, R.E., 2002. Is road rage a serious traffic problem? Traffic Inj Prev. 3, 183-189.

52. Smart, R.G., Asbridge, M., Mann, R.E., 2003. Psychiatric distress among road rage victims and perpetrators. Can J Psychiatry. 48, 681-688.

53. Smart, R.G., Mann, R.E., Zhao, J., 2005. Is road rage increasing? Results of a repeated survey. J Safety Res. 36, 195-201.

54. DePasquale, J.P., Geller, E.S., Clarke, S.W., Littleton, L.C., 2001. Measuring road rage: Development of the Propensity for Angry Driving Scale. J Safety Res. 32, 1-16.

55. Deffenbacher, J.L., Oetting, E.R., Lynch, R.S., 1994. Development of a driving anger scale. Psychol Rep. 74, 83-91.

56. Deffenbacher, J.L., Lynch, R.S., Oetting, E.R., Swaim, R.C., 2002. The driving anger expression inventory: A measure of how people express their anger on the road. Behav Res Ther. 40, 717-737.

57. Smart, R.G., Stoduto, G., Mann, R.E., Adlaf, E.M., 2004. Road rage experience and behavior: Vehicle, exposure, and driver factors. Traffic Inj Prev, 5, 343-348. 
58. DRUID (Driving Under the Influence of Drugs, Alcohol and Medicines), Prevalence of alcohol and other psychoactive substances in drivers in general traffic. Part I: general results, deliverable D 2.2.3. Part 1, Working Paper, 2011. http://www.druidproject.eu/ (accessed December 2014).

59. López, M., 2012. Dirección General de Tráfico (DGT). Las drogas bajo Control.

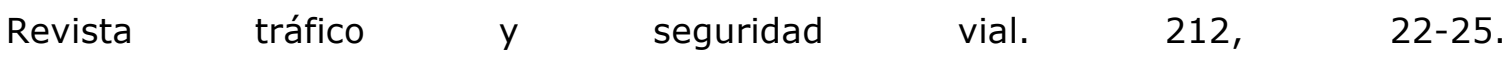
http://www.dgt.es/revista/num212/pdf/revista-trafico-y-seguridad-vial-numero-212completa.pdf (acceso 20/01/15)

60. Houwing, S., Hagenzieker, M., Mathijssen, R., Bernhoft, I.M., Hels, T., Janstrup, K., Van der Linden, T., Legrand, S.A., Verstraete, A., 2011. Prevalence of Alcohol and Other Psychoactive Substances in Drivers in General Traffic. Part I: General Results and Part II: Country Reports, DRUID Deliverable 2.2.3. Available on line at: http://www.druid-project.eu/Druid/EN/deliverales-list/downloads/Deliverable_2_2_3 _Part1.pdf?_blob=publicationFile and http://www.druid-project.eu/Druid/EN/ deliverables-list/downloads/Deliverable_2_2_3_Part2.pdf?__blob=publicationFile (acceso 09/06/14).

61. Mathijssen, R., Houwing, S., 2005. The prevalence and relative risk of drink and drug driving in the Netherlands: a case-control study in the Tilburg pólice district. Leidschendam: SWOV. http://www.swov.nl/rapport/r-2005-09.pdf (acceso $12 / 02 / 15)$.

62. Álvarez, F.J., Fierro, I., 2010. El consumo de drogas en Castilla y León, 2008. Valladolid, Spain: Junta de Castilla y León, Consejería de Familia e Igualdad de Oportunidades.

63. Rodríguez-Martos, A., Navarro, R., Vecino, C., Pérez, R., 1986. Validación de los cuestionarios KFA (CBA) y CAGE para diagnóstico del alcoholismo. Drogalcohol, 11, 132-139. 
Capítulo 2

Prevalence of psychoactive substances, alcohol, illicit drugs, and medicines, in Spanish drivers: a roadside study

Gómez-Talegón, T., Fierro, I., González-Luque, J.C., Colás, M., López-Rivadulla, M., Álvarez, F.J.

Forensic Sci Int, 2012; 223: 106-113 



\section{Abstract}

Following population, geographic, road type and time criteria, Spain has carried out random, roadside controls of 3302 representative sample of Spanish drivers, including saliva analysis for 24 psychoactive substances and alcohol breath tests. The $81.4 \%$ of the drivers were male, with an average age of $34.8 \pm 11.8$ (mean $\pm \mathrm{SD}$ ). The $17 \%$ of the drivers were found to be positive to any of the substances analysed. The $6.6 \%$ of the drivers found positive to alcohol ( $>0.05 \mathrm{mg} / \mathrm{l}$ in breath), $11 \%$ were found positive to any illicit drug, and $2 \%$ were positive to one of the medicines analysed. Some drivers were positive in more than one substance. The most common illicit drugs among Spanish drivers were cannabis $(7.7 \%)$, or cocaine $(3.5 \%)$, either alone or combined with other substances. The most prevalent medicines were the benzodiazepines (1.6\%). As a tendency, higher figures for positive cases were observed among males than in females (being statistically significant the differences for alcohol, cannabis and cocaine). Alcohol and cocaine positive cases were more frequently found among drivers of urban roads. Alcohol positive cases (alone, $>0.05 \mathrm{mg} / \mathrm{l}$ ), were more likely found as age increase $(O R=1.02)$, those driving in urban roads $(O R=2.13)$, and driving at any period than weekdays, while alcohol + drugs cases were more likely found among males $(O R=2.819)$, those driving on urban road $(O R=2.17)$ and driving at night periods. Finding a medicines positive case was more likely as elder the driver was $(O R=1.05)$. There have been differences in the prevalence of positive cases of alcohol, cannabis and cocaine, in relation to the period of the week: in three cases the highest prevalence seen in night time. This study shows the high prevalence of psychoactive substances and alcohol in Spanish drivers, mainly illicit drugs (cannabis). This question requires a response from the authorities and from society, with an integral and multi-disciplinary approach that can heighten the population's awareness of the risks of driving under the influence of certain substances.

\section{Keywords}

Drivers; Substance abuse detection; Street drug testing; Saliva; Breath tests 



\section{Introduction}

There is continuously increasing concern about driving under the influence of illicit drugs and medicines in the road of the EU, although it is recognized that alcohol is the most prevalent substance detected among drivers on European roads [1].

In a recent review [2] of roadside surveys in drivers published between 1997 and 2007 [3-7] provide data for eight countries, reporting that the prevalence of drug-positive drivers ranged from about $1 \%$ to $15 \%$. Driving under the influence of a combination of alcohol and drugs is not uncommon, being estimated that this occurs in about $0.3-1.3 \%$ of the general driving population [2]. As a single substance, cannabis tends to be the most frequently detected illicit drug in 6 of the 7 analysed studies [3-9]. However, there are striking differences between studies: from one side, these could be related to the various designs of the studies as well as due to methodological issues (substances analysed, cut-offs, etc.), sample size (within the IMMORTAL study [7] stopped and tested drivers were 410 for Norway, 1312 for UK and 3799 for The Netherlands), and from other side, due to the possible differences between countries: for example, recent report from Norway [8,9] indicated that benzodiazepines (1.4\%) and Zhypnotics (zopiclone $=1.4 \%$ ) are frequently found in drivers, even in higher concentrations that those legally proposed (for example $25 \%$ of zopiclone cases were over the legal limit, while this figure was $70 \%$ for diazepam). In the United Kingdom [7], 3,4-methylenedioxyamphetamine $\quad$ (MDA), 3,4methylenedioxyethylam-phetamine (MDEA) and MDMA were the illicit drugs more prevalent $(4.6 \%)$.

In Spain, statistics show that illicit drugs and alcohol use is quite prevalent in the Spanish population.

We are the first European country in prevalence for the consumption of cocaine "lifetime" (10.2\%), "last year" (2.7\%) and "last month" (1.3\%) and also the first for consumption of cannabis the last month (7.6\%) [10]. Several studies have shown that drivers tend to drive after illicit drug use. In a report on cannabis use and driving [11], 9.7\% of drivers reported driving a vehicle under the influence 
of cannabis. Data from 5745 blood samples of 59,071 drivers killed on the Spanish roads in the period 1991-2000 show that in $50.1 \%$ of cases some type of psychoactive substance was detected; $43.8 \%$ alcohol, $8.8 \%$ illicit drugs and 4.7\% medicines [12]. Data from the 2010 based on 855 blood sample analyses of 1511 drivers killed in traffic road accidents show that alcohol, illicit drugs or medicines were detected in $363(42.5 \%)$ of cases [13]. The more prevalent substance was alcohol (205 cases), medicines (45 cases), illicit drugs ( 37 cases), alcohol and illicit drugs (50 cases), illicit drugs and medicines (16 cases), alcohol and medicines ( 6 cases) and alcohol, illicit drugs and medicines (4 cases) [13].

In Spain, it is considered a criminal offence against road safety regulations to drive a vehicle under the influence of alcohol $(>0.5 \mathrm{~g} / \mathrm{l}$ in blood or $>0.25 \mathrm{mg} / \mathrm{l}$ in breath, these limits are $>0.3 \mathrm{~g} / \mathrm{l}$ in blood or $0.15 \mathrm{mg} / \mathrm{l}$ in breath, for novice and professional drivers), and also under the influence of illicit drugs or certain medicines. It is also an offence to refuse to participate in the legally established tests to prove the presence of such substances (articles 379.2 and 383 of the Penal Code and articles $20,21,27$ and 28 of the General Traffic Regulations) $[14,15]$. Such offences are considered to be very serious and may result, according to the current regulations, apart from the corresponding fine, also in the suspension of the offender's driving licence for up to three months and, if the penal code is applied, even a prison sentence of between 3 and 6 months, or a fine of between 6 and 12 months, as well as community work of between 31 and 90 days and, in any case, a prohibition to drive of between 1 and 4 years $[14,15]$.

In order to clear up all these aspects, several European countries have, within the European Union granted DRUID project, carried out epidemiological studies, using similar methodologies, on the presence of alcohol/drugs/medicines in drivers in 13 European countries, including Spain (http://www.druid-project.eu) [16]. Spain has participated in this project, making random analyses for the DRUID project of the presence of substances in 3226 car drivers (according to DRUID agreements, vehicles of over $3500 \mathrm{~kg}$ and motorcyclists, were not included in the study [16]). The study presented in this paper has analysed 3407 cases, as motorcyclists have also been included. The reason of including motorcyclist, it is that is quite frequent in Spain. 
The aim of this study is to assess the prevalence of psychoactive substances, alcohol, illicit drugs and certain medicines, positive cases in the oral fluid of a nationwide representative sample of the general population of drivers in Spain.

\section{Material and methods}

\subsection{Study design and field trial}

\section{Target population}

The target population was motor vehicle drivers, except bikes and vehicles of over $3500 \mathrm{~kg}$, on Spain's public roads, both rural and urban.

\section{Study design}

It was designed as a representative, cross-sectional, nationwide study. In order to select the police random roadside check points, in a first step, the country was divided in four areas ("Cantábrica", "Norte", "Mediterránea", and "Sur"). In a second step, for each one these four areas, eight rural and eight urban roads (in total 32 roads) were randomly selected based on the population size of the municipalities they link, less than 20,000 inhabitants, 20000-99999 inhabitants, 100000-499999, and 500000 and over inhabitants of the municipalities. Later, for each one of these roads, four roadside check points were randomly selected (in total 128 police roadside check points). Finally, in each one of these 128 police roadside check points, the number of police periods of roadside checks was randomly determined based on the population size of the municipality; in total 731 periods of police random roadside checks were established. Each random roadside check was determined to be 2-3 h long, and checking a maximum between 4 and 6 drivers.

The study was planned to be carried out along a full year, between September 26th, 2008 and August 24th, 2009, taking into account also the periods of banknational holidays and not holidays, as well as taking into account four periods, on different days of the week and hours of the day (a) Monday-Friday from 7:00 to 23:59 h (weekdays); (b) Monday-Friday from 0:00 to 6:59 h (weeknights); (c) 
Saturday, Sunday and holidays from 7:00 to 23:59 h (weekend days); (d) Saturday, Sunday, Monday and holidays from 0:00 to 6:59 h (weekend nights). The number of valid random roadside checks with valid samples (oral fluid) was established in 3000. This number of cases supposes aggregate results, for the population as a whole, with a confidence level of $95 \%$ and an accuracy of within $1 \%$. It should be noted that the study was designed to be nationwide representative, but not regional (17 regions in Spain) representative.

\section{Ethical issue}

The study was approved by the Research Ethics Committee of the University of Valladolid on January 31st, 2007.

\section{Sample weighting: traffic intensity}

The data have been weighted according to the traffic intensity. As there is no full national information on traffic intensity in Spain, at each one of the 128 points selected for the study, traffic intensity was monitored along the year of study, in accordance with the usual methodology in such cases [17].

\section{Positive cases}

The analytical results were expressed as the previous studies $[12,18]$ : (i) negative, no substance over at the cut-offs established in oral fluid, or (ii) positive when some type of substance in oral fluid was over at the cut-offs established. Once a substance had been found, samples were classified in the excluding following groups: (i) alcohol alone, (ii) alcohol + medicine(s), (iii) alcohol + illicit drug(s), (iv) alcohol + medicine(s) + illicit drug(s), (v) illicit drug(s) alone, (vi) medicine(s) alone, and (vii) illicit drug(s) + medicine(s). In a second classification, positive cases were classified as (i) alcohol alone or in combination, (ii) medicines alone or in combination, and (iii) illicit drugs positives cases alone or in combination. In this second classification, positives cases were not exclusive, and, as some drivers were found to be positive to more than one substance, so the sum of the percentages of the individual analyses for alcohol, drugs and medicines is higher than the total number of positive cases for any one substance. 


\section{Field trial and data gathering}

The present study was included within the random roadside checks carried out by the traffic police. Two researchers collaborated in the field trial with the police officers in each of the 731 sessions of police random roadside checks of the study. Roadside police controls were legally regulated and mandatory [15]. Furthermore, in the case of this study, specific internal instructions were emitted by the National Traffic Directorate (08/S-102). Therefore, within each one of the random roadside checks, there were carried out the activities done by the traffic police, as well as the activities done by the researchers, like obtaining the subject informed consent, etc. In this study, it was mandatory to submit to an alcohol breath test, and an on-road analysis of saliva using the "Dragger 5000 test" device, which determines 6 substances with the following cut-off point: opioids (morphine), $20 \mathrm{ng} / \mathrm{ml}$; amphetamines (D-amphetamine), $50 \mathrm{ng} / \mathrm{ml}$; meta-amphetamines (D-meta-amphetamine), $35 \mathrm{ng} / \mathrm{ml}$; cocaine (cocaine), $20 \mathrm{ng} / \mathrm{ml}$; THC (Delta-9-THC), $25 \mathrm{ng} / \mathrm{ml}$; benzodiazepines (diazepam), $15 \mathrm{ng} / \mathrm{ml}$. It is an offence to refuse to participate in these tests $[14,15]$. The voluntary participation was requested while drivers were waiting for the result of the mandatory saliva test performed by the police, and a second saliva sample was obtained for the analysis of substances in the laboratory. Notice that if the breath test and/or the saliva test with Dräger 5000 were positive, legal prosecution followed and it was then mandatory to provide the second saliva sample. This procedure was selected in order to minimize the refusals to participate.

The following driver data was registered: (i) sociodemographic data (gender, age, nationality), and (ii) driving patterns (type of vehicle, if have a driver's license and the class of driving license, and the weekly average kilometers driven the previous year).

\subsection{Biological samples and toxicological aspects}

The biological sample was always taken by traffic police. The oral fluid sample (approximately $1 \mathrm{ml}$ ) was taken using the Statsure Saliva Sampler device and sent to the laboratory for analysis, in a special container at a temperature between 2 and $8{ }^{\circ} \mathrm{C}$, within $36 \mathrm{~h}$ of collection. The relationship between the 
amount of saliva and the buffer provided by the manufacturer is $1: 1$. Despite this, the tube was weighed and subsequent calculations were made taking into account the dilution factor, and the actual value measured by weighing.

A total of 24 substances (Table 1) were analysed using the liquid chromatography-tandem mass spectrometry technique as described previously [19]. All the saliva samples were analysed in the same laboratory of the forensic toxicology service of the University of Santiago de Compostela. Regarding cutoffs, we have followed the DRUID criteria [16, page 18]: "For each substance an analytical cut-off has been selected based on the lowest limit of quantification (LOQ) that could be measured by all toxicological laboratories that were involved in the analysis of the substances. LOQ's reflect the lowest concentrations for substances at which quantitative results can be reported with a high degree of $95 \%$ confidence. For the final results presented in this report, equivalent cutoffs, and not the LOQ's, are used for analysis of the core substances to correct for differences in concentrations of substances in blood and in saliva. The reason for this is that for many substances the concentrations in oral fluid are much higher than in blood, while for some compounds the concentrations are lower $[20,21]$. This means that if LOQ's for oral fluid samples were used to collect information on recent drug use, the prevalence for most substances would probably have been higher than that it would have been the case if blood was used as a sampling matrix [16, page 18]".

After obtaining the oral fluid sample, the drivers were subjected to an alcohol breath test using the Dräger(c) Alcotest 6810 device. When a positive result was obtained, in accordance with the national legal requirements, a second breath test was performed using the Dräger(C) Alcotest 7110 MKIII, for the purpose of legal evidence. Alcohol was not analysed in oral fluid. 
Table 1 Analysed substances and cut-off points determining positivity according with DRUID criteria [16].

\begin{tabular}{|c|c|c|}
\hline Substance & Oral fluid analytical cut-off $(\mathrm{ng} / \mathrm{ml})$ & Breath $(\mathrm{mg} / \mathrm{l})$ \\
\hline Ethanol & --- & 0.05 \\
\hline 6-Acetilmorphine & 16.0 & \\
\hline Alprazolam & 3.5 & \\
\hline Amphetamine & 360.0 & \\
\hline Benzoilecgonine & 95.0 & \\
\hline Clonazepam & 1.7 & \\
\hline Cocaine & 170.0 & \\
\hline Codeine & 94.0 & \\
\hline Diazepam & 5.0 & \\
\hline Flunitrazepam & 1.0 & \\
\hline Lorazepam & 1.1 & \\
\hline MDA & 220.0 & \\
\hline MDEA & 270.0 & \\
\hline MDMA & 270.0 & \\
\hline Metadone & 22.0 & \\
\hline Metamphetamine & 410.0 & \\
\hline Morphine & 95.0 & \\
\hline Nordiazepam & 1.1 & \\
\hline Oxazepam & 13.0 & \\
\hline THC & 27.0 & \\
\hline Zolpidem & 10.0 & \\
\hline Zopiclone & 25.0 & \\
\hline Tramadol & 480.0 & \\
\hline 7-Amino-clonazepam & 3.1 & \\
\hline 7-Amino-flunitrazepam & 1.0 & \\
\hline
\end{tabular}




\subsection{Data analysis}

A database of an anonymous nature was generated. As earlier mentioned, the data were weighted in accordance with their exposition to the traffic at each of the check points. Percentages are shown with their Confidence Interval at 95\%, while for the continuous variables the mean $\pm S D$ is shown. The comparisons between two groups were carried out using the Student- $T$ test in the continuous variables and the Chi-squared test in the category variables. The correlation (linear and/or polynomial correlation) between the positive cases and age has been analysed. Logistic regression have been carried out to evaluate the influence of gender (male/female), road type (urban/rural), period of the week (weekdays, weeknights, weekend days, weekend nights) and exposure (Kilometers driven per week) on positive cases. Values of $P \leq 0.05$ have been considered statistically significant. The statistical package SPSS 18.0 has been used for the analyses.

\section{Results}

\subsection{Characteristics of drivers involved in the study}

Of the 3407 drivers stopped at random, 3302 were included in the study and 105 were rejected for one of the following reasons: refusal - not giving consent to providing the sample $(n=63)$, not obtaining a sample due to a lack of saliva $(n=2)$, not fulfilling the inclusion criteria $(n=40)$.

Of the 3302 drivers, the $81.5 \%$ were male. Almost 9 out every 10 were Spanish and were driving a car, with a weekly average over the previous year of $448.1 \pm 663.2 \mathrm{~km}$ driven. The average age was $34.8 \pm 11.9$ (Table 2). Females are younger than males, drove less kilometre per year, and are more likely Spaniards and driving a car (Table 2). 


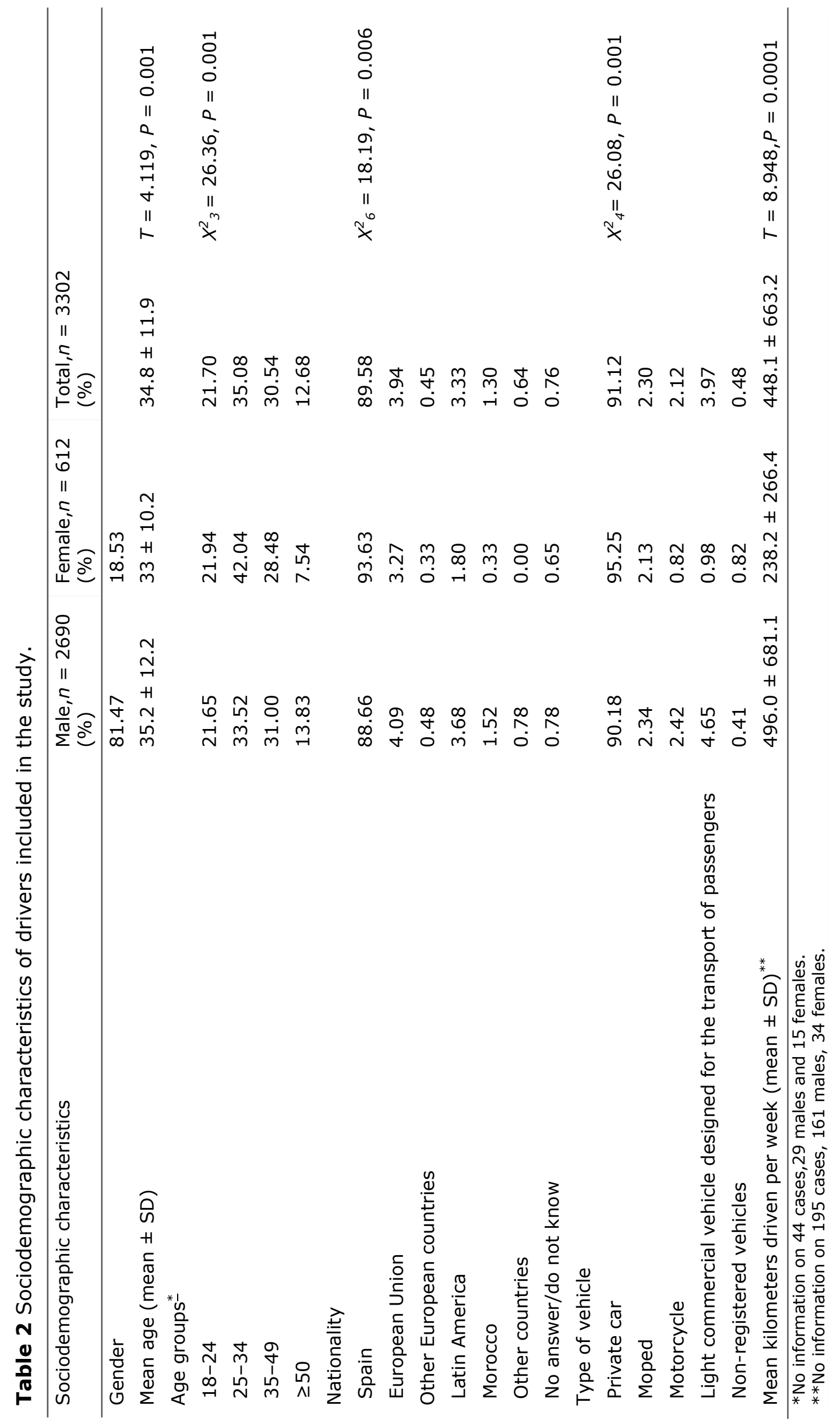




\subsection{Prevalence of positive cases}

The $17 \%$ of the drivers ( $95 \% \mathrm{CI}, 15.7-18.2)$ were found to be positive to any of the substances analysed (Figure 1). The 6.6\% (95\%CI, 5.7-7.4), of the drivers were found positive to alcohol ( $>0.05 \mathrm{mg} / \mathrm{l}$ in breath), $11 \%$ (95\%CI, 9.8-12) were positive to any illicit drug, and $2 \%(95 \% \mathrm{CI}, 1.5-2.4)$ were positive to one of the medicines analysed. Notice that some drivers were positive in more than one substance (Figure 1).

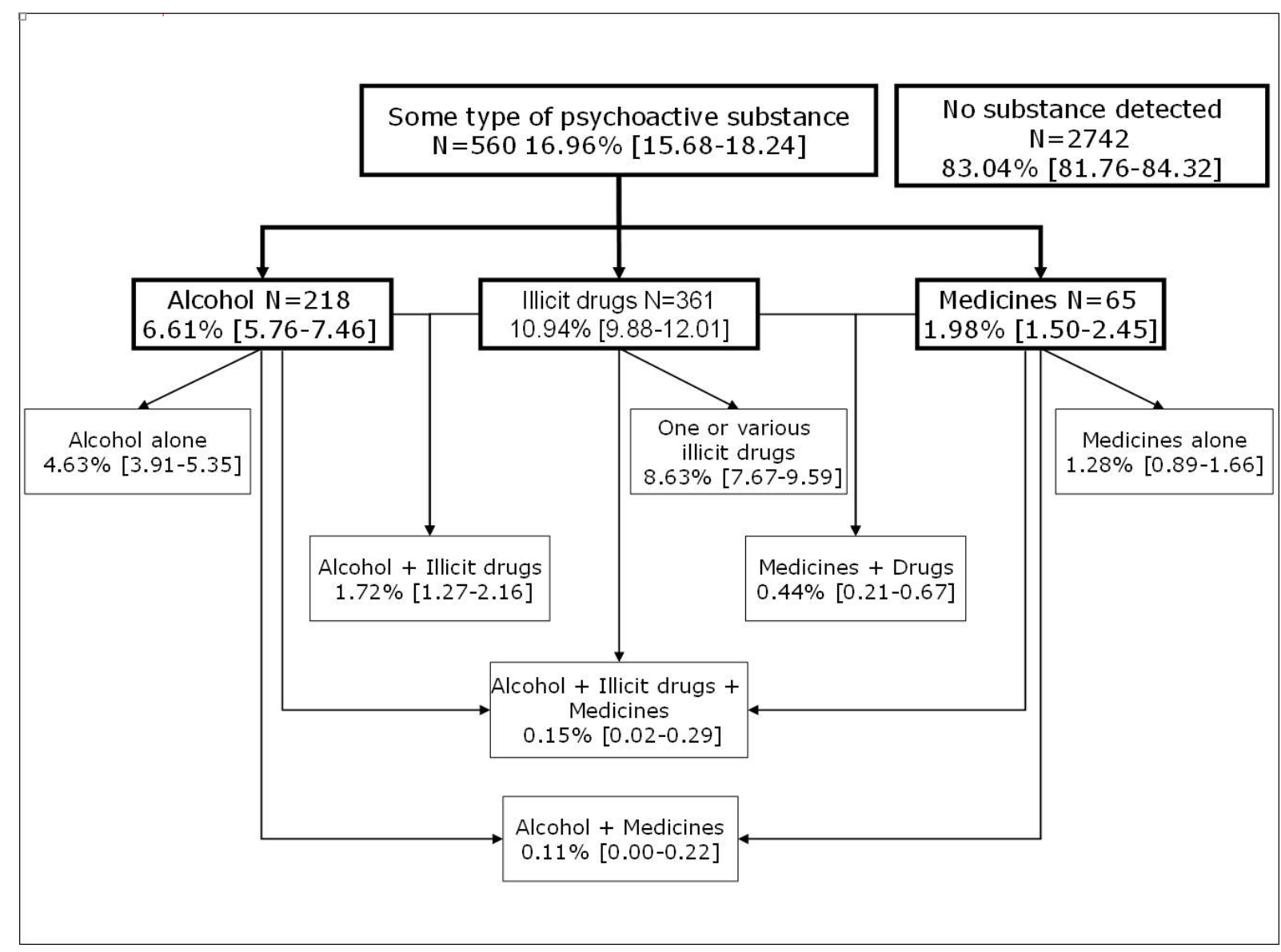

Figure 1 Cases found positive for alcohol, illicit drugs, and medicines in Spanish drivers. Results are presented al percentage and 95\% Confidence Interval.

Regarding alcohol, in about $70 \%$ of alcohol positive cases was detected alcohol alone $(4.6 \%$ out of $6.6 \%)$, while the most frequent combination was with illicit drugs. The $31 \%$ of those found positive to alcohol were over the legal limits for alcohol allowed in Spain (>0.25 mg/l in breath). For illicit drugs positive cases, in most cases (about $80 \%$ ) it is finding one or various illicit drugs ( $8.6 \%$ out of 
$11 \%$ ) without alcohol or medicines. In about two out of every tree positive cases to medicines were only detected medicines (Figure 1).

Logistic regression analysis shows that finding a substance positive case driver decreased with age $(O R=0.98)$, and was more likely among males $(O R=2.77)$, those driving on urban road $(O R=1.80)$, and driving at any period than weekdays (Table 3 ). Alcohol positive cases (alone, $>0.05 \mathrm{mg} / \mathrm{l}$ ), were more likely found as age increase ( $O R=1.02)$, those driving in urban roads ( $O R=2.13$ ), and driving at any period than weekdays. There was a likelihood of finding a driver positive case for drugs alone among males ( $O R=5.52)$, those driving urban road $(O R=1.52)$, and as younger the driver was $(O R=0.929)$. Alcohol + drugs cases were more likely found among males (OR $=2.819)$, those driving on urban road $(\mathrm{OR}=2.17)$ and driving at night periods. Finding a medicines positive case was more likely as elder the driver was $(O R=1.05)$ (Table 3). In no case exposure (km drivers) showed any effect. Figure 2 shows the linear correlation between age and prevalence of driver's positive cases to any substance. 


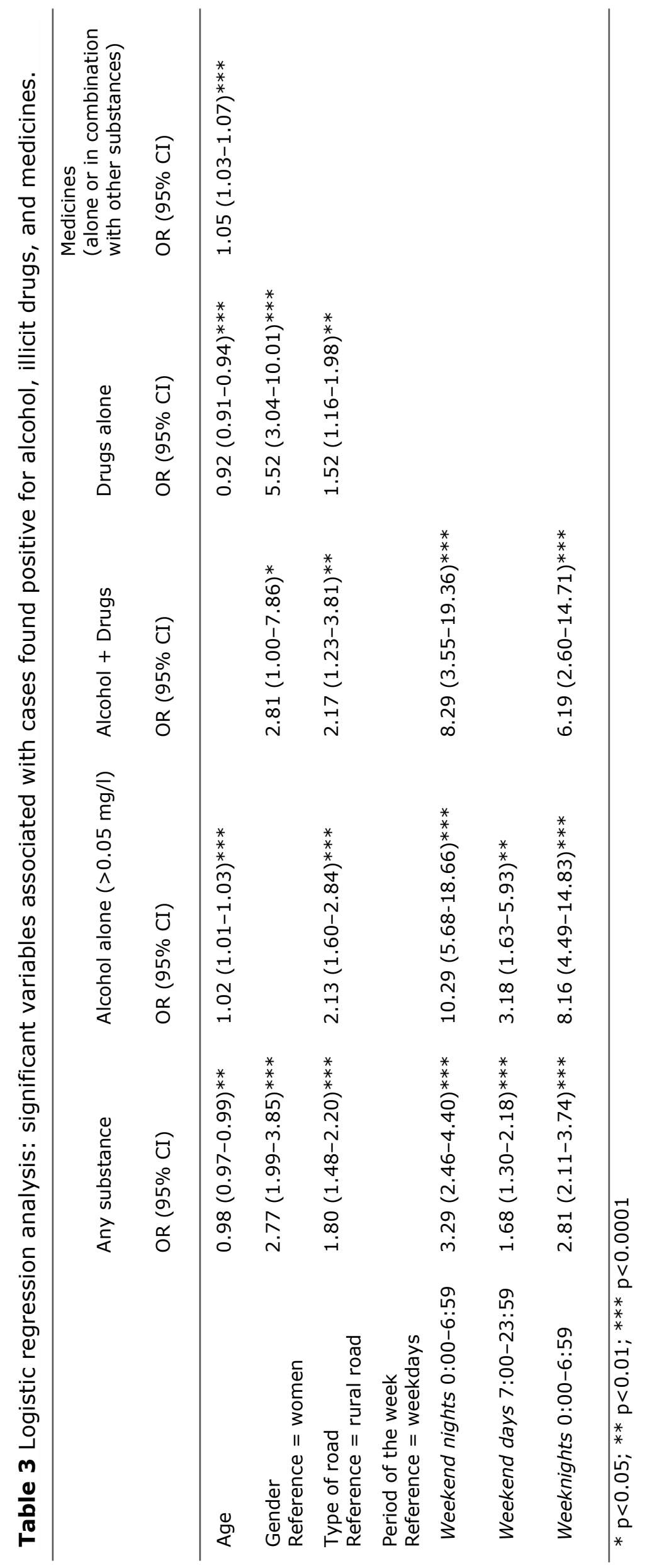




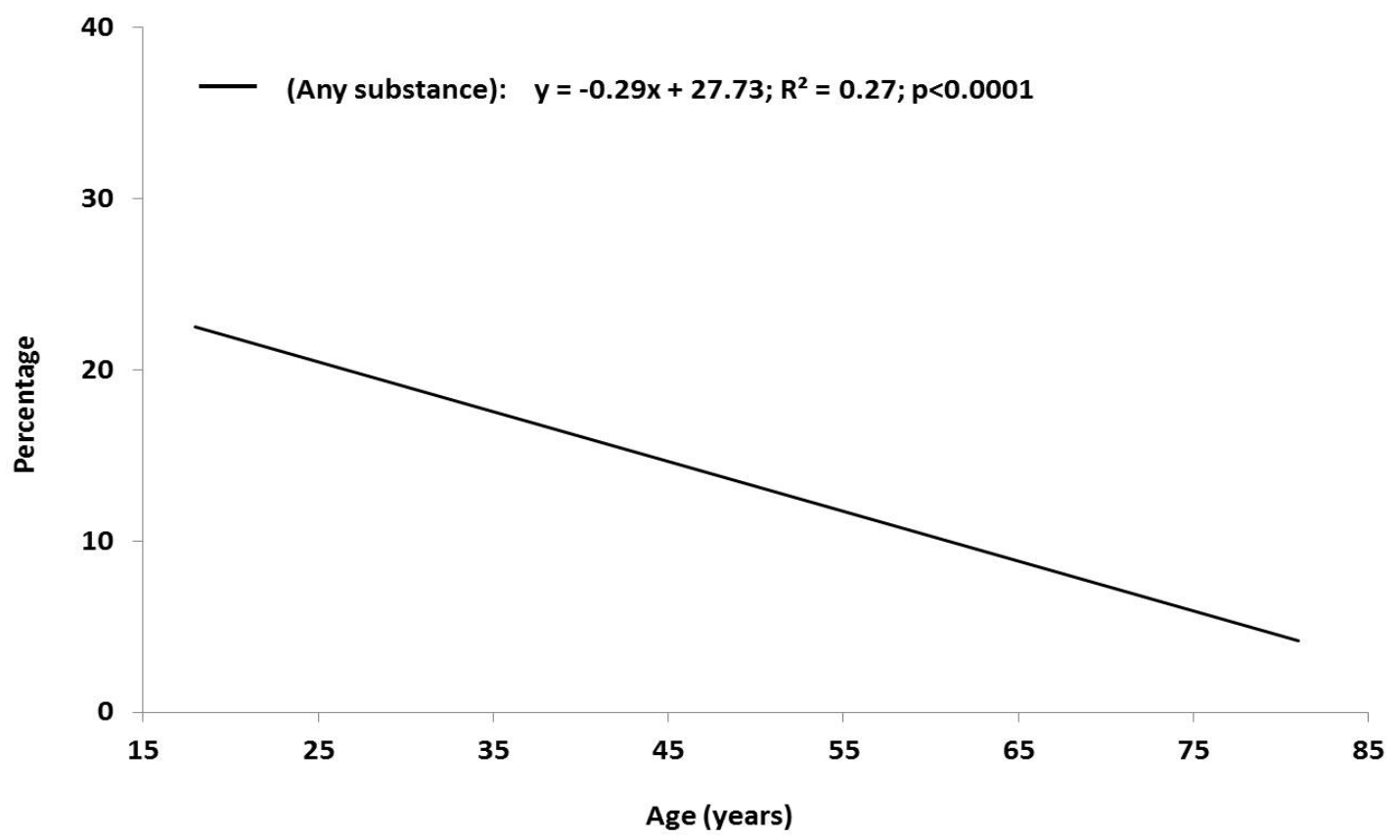

Figure 2 Correlation between positive cases to any substance with age.

\subsection{Types of substances detected}

Cannabis $(7.7 \%)$ was the most frequently detected illicit drug among Spanish drivers, followed by cocaine (3.5\%) (Table 4). Among positive cases to medicines, benzodiazepines were the most prevalent among Spanish drivers $(1.6 \%)$. Medicinal opioids were found much less frequently $(0.45 \%)$. No case was found to be positive to Z-hypnotics (Table 4). In all cases figures were for the presence of the various substances alone or in combination with other substances. 


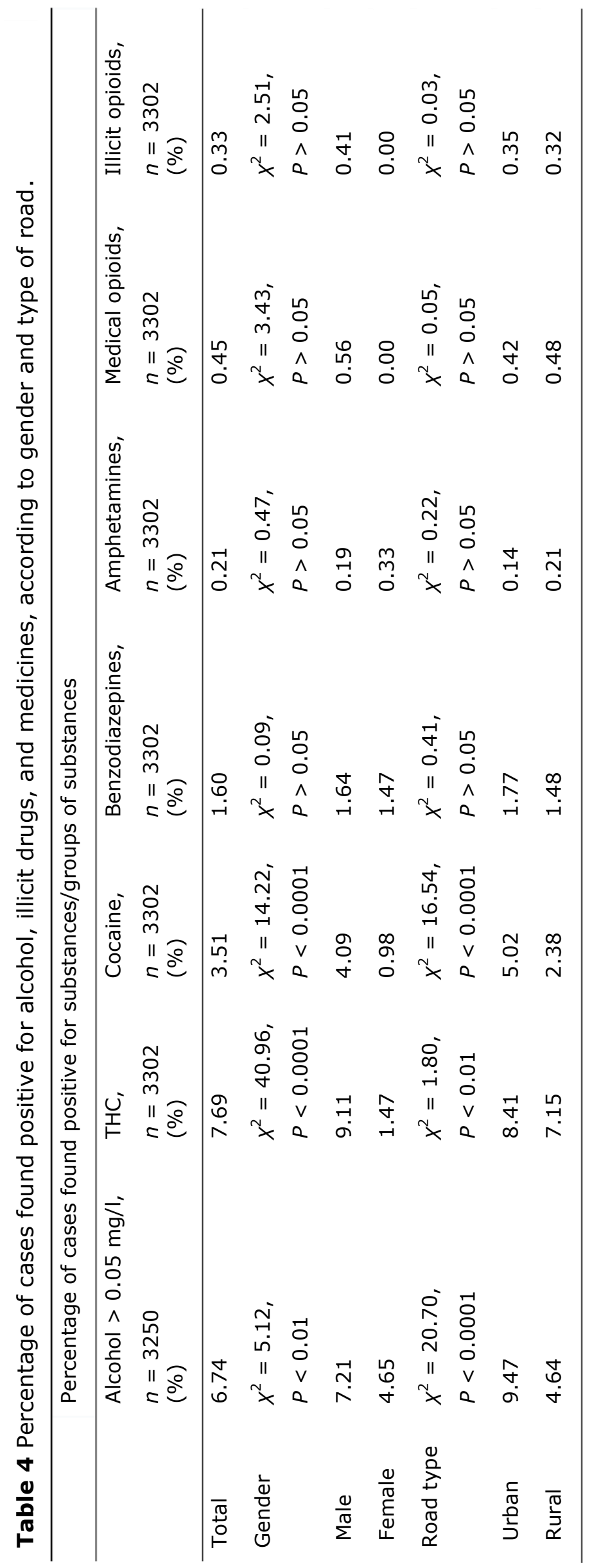


As a tendency, higher figures for positive cases were observed among males than in females (being statistically significant the differences for alcohol, cannabis and cocaine). Alcohol and cocaine positive cases were more frequently found among drivers of urban roads (Table 4). We have not found (Figure 3) correlation between alcohol positive cases (alone or in combination) and age: however, alcohol alone positive cases increased with age (see also Table 3), while alcohol + drugs positive cases decreased with age. For cannabis, cocaine and benzodiazepines the relationships within positive cases and age was polynomial: for cocaine and cannabis there is a tendency to decrease with age, and for benzodiazepines to increase with age (Figure 4).

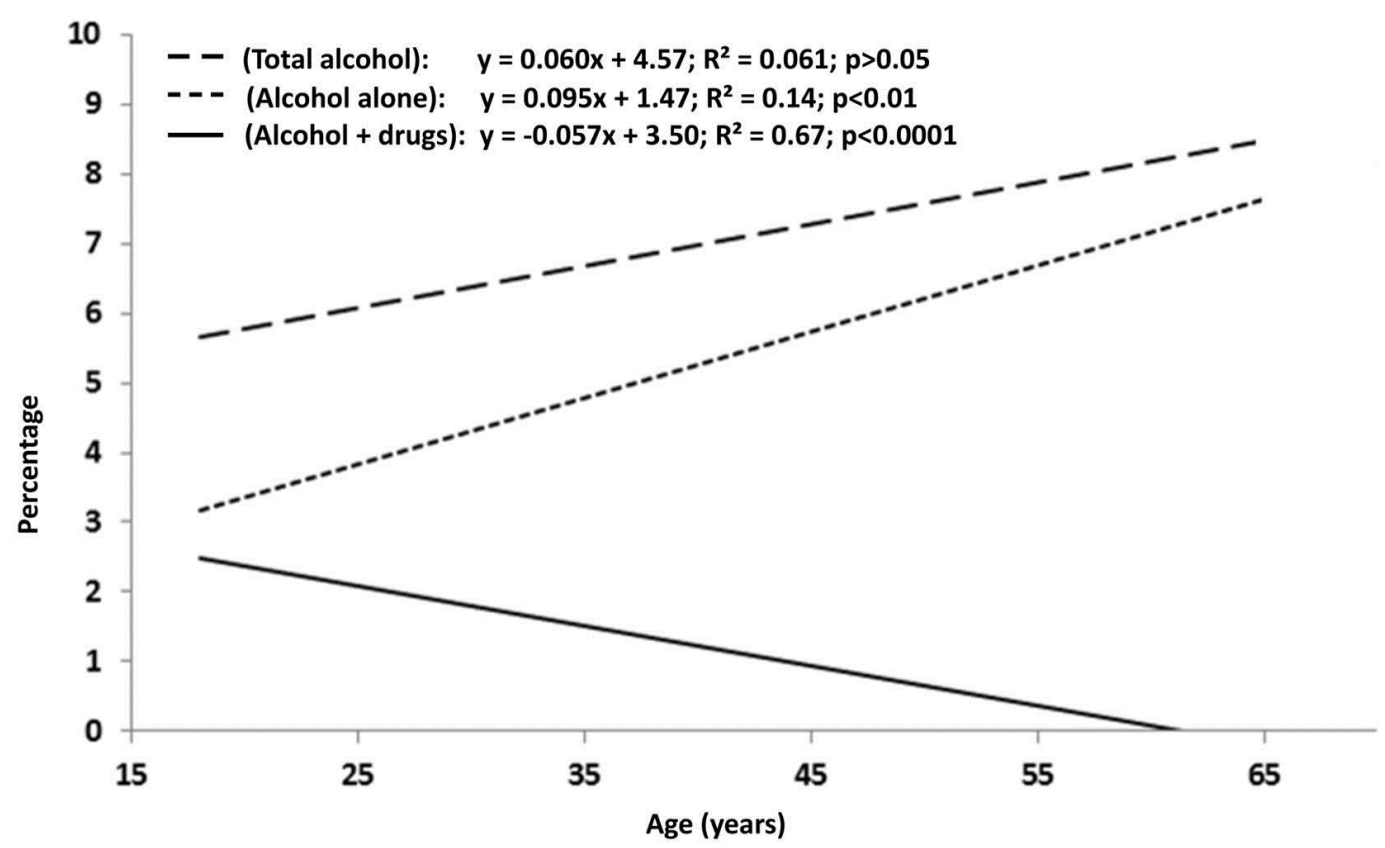

Figure 3 Correlation between alcohol $(<0.05 \mathrm{mg} / \mathrm{l})$ positive cases in breath with age.

Figure 5 shows prevalence of positive cases with regard period of the week, being observed differences in the case of alcohol, cannabis and cocaine: in all these three cases higher prevalence were observed in the night periods. 


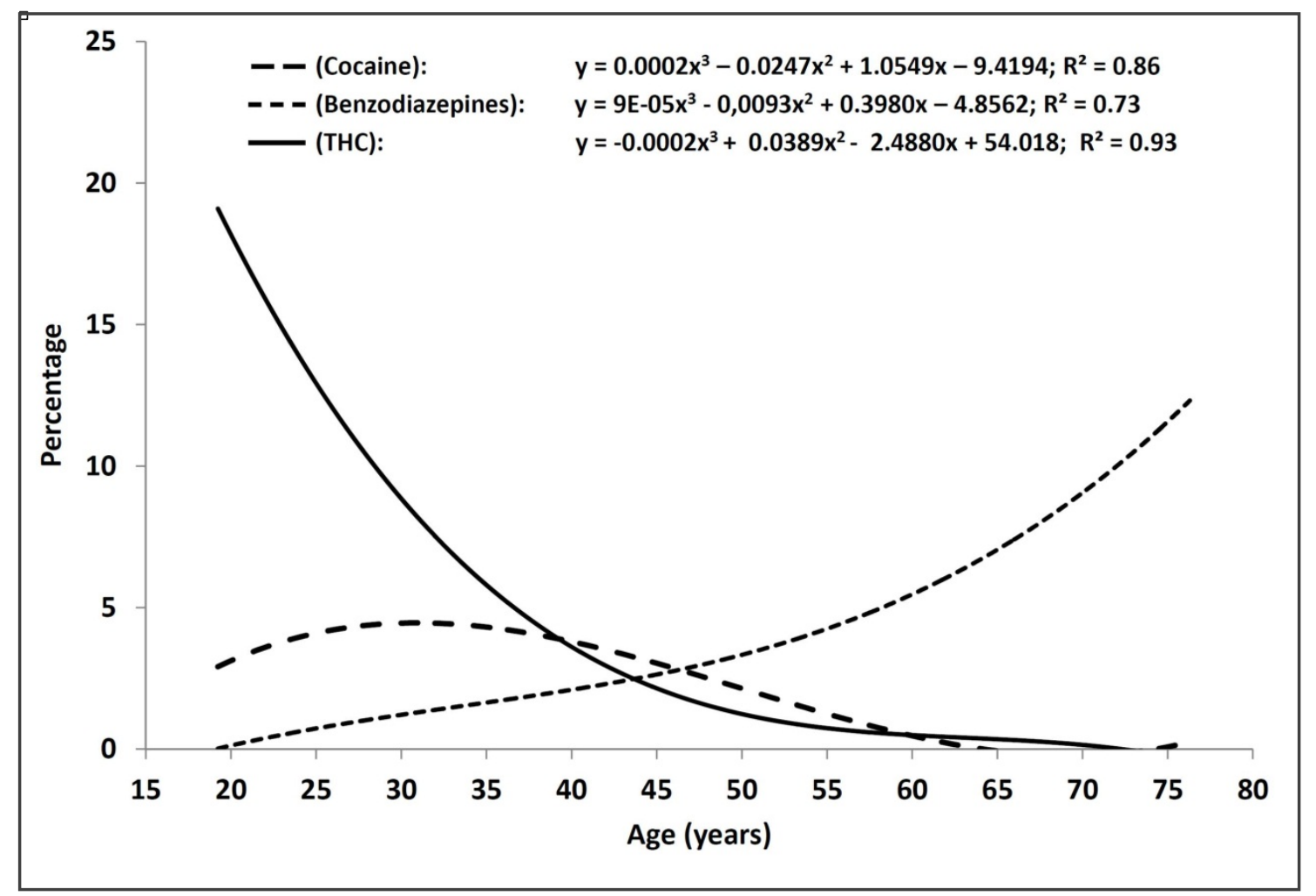

Figure 4 Correlation between cannabis, cocaine and benzodiazepines positive cases with age (in all cases alone or in combination with other substances).

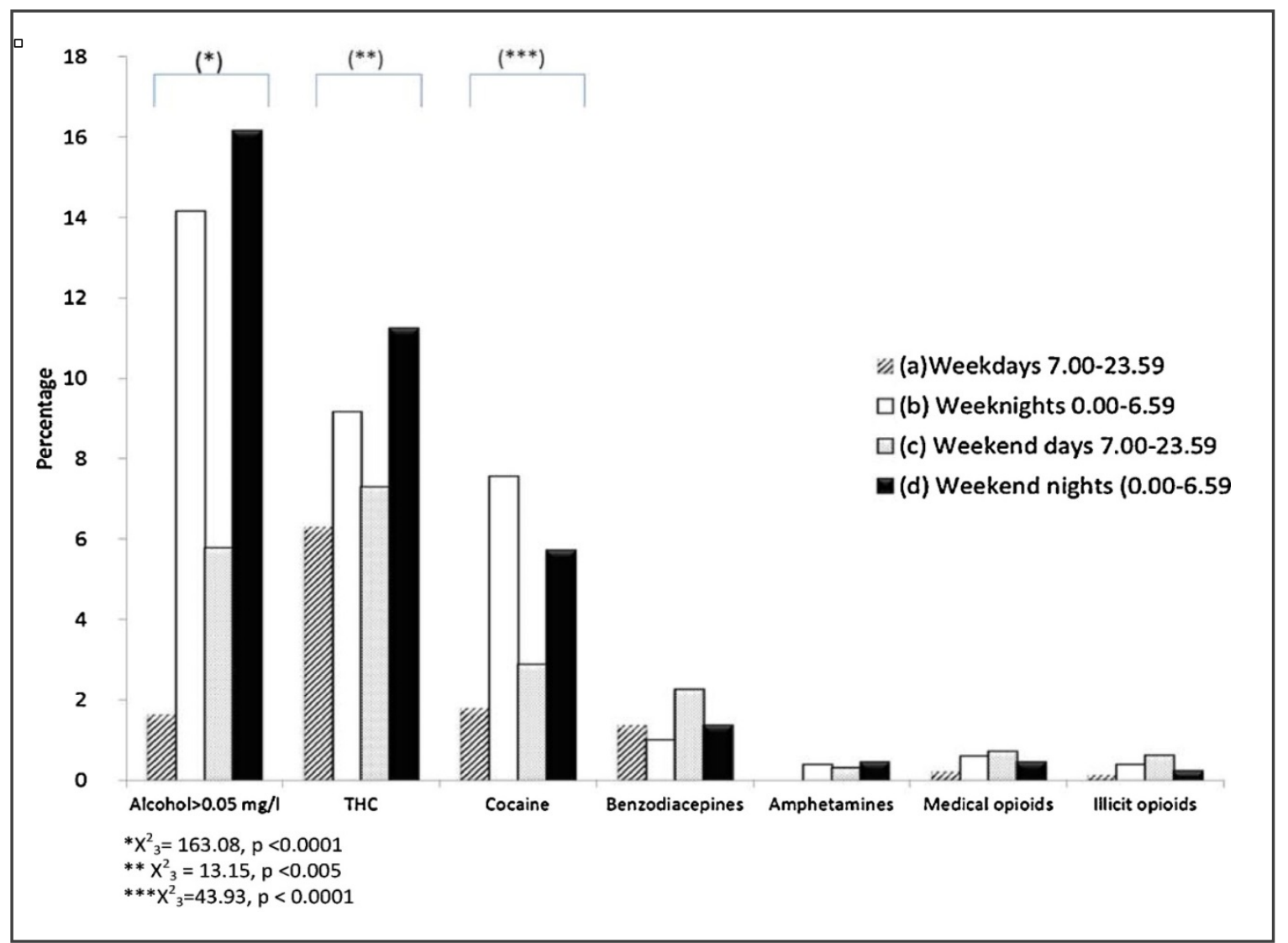

Figure 5 Distribution of positive cases according substance types and periods of the week. 


\section{Discussion}

This roadside study shows a series of interesting data: (i) driving after consuming psychoactive substances is a frequent occurrence in Spain, involving almost 1 in every 6 drivers; (ii) in Spanish drivers, the prevalence of illicit drugs ( 1 in 10, mainly cannabis and cocaine) is greater that of alcohol; (iii) THC (cannabinoids) was the most detected substance, present in 1 out of every 12 drivers, followed by alcohol in 1 out of every 15; (iv) almost $2.5 \%$ of the drivers were found positive for more than one substance; $(v)$ the positive cases occurred more frequently in men, on urban roads and at weekend nights and decreased with age of driver.

The global results obtained in the DRUID project (13 countries) show that Spain is the country with the highest prevalence of drivers found to be positive for illicit drugs at almost $11 \%$, followed by Italy (3.9\%), the Netherlands $(2.5 \%)$, and Portugal (1.8\%). The combination of alcohol and drugs was also most prevalent in Spain (1.7\%), followed by Italy (1\%) [16].

The analysis of substances in oral fluid has been largely studied [22] and used and carried out previously in other roadside studies, in Canada [4], Denmark [5], Australia [6], Norway [7-9], Germany [23], and Scotland [24], some of them has also been analysed the presence of substances in others biologic fluids (blood/urine). However, comparison with these studies is difficult due to the different methodologies used for the sample selection, the different techniques for taking oral fluid or blood samples, and the different cut-off points and analytical methods used. Taking this into account, the proportion of drivers found positive to substances (16.6\%) from our study is similar to the $16.8 \%$ found by Wylie in United Kingdom [24], but much higher than that reflected by other authors (between $10 \%$ and $2 \%$ ) $[3,5,6,8]$. It should be noted that in all the roadside studies consulted by us, except that carried out in Australia [6], the participation of the drivers was voluntary, while it was obligatory in our study.

As for the different substances, THC is the substance most prevalent among Spanish drivers and Spain is the European country participating in the DRUID project with the highest prevalence of drivers found positive for THC (7.7\%), four 
times higher than for the next highest (Netherlands, 2.1\%), and much higher than the average for the DRUID study (1.3\%) [16]. This agrees with the figures for cannabis use in the general population, Spain is the European country with the highest prevalence of cannabis use the last month in the general population in all age ranges (7.6\%) (aged 15-64 years) [10], and also present the highest prevalence of consumption the last month among the younger population, $14.1 \%$ (aged 15-34 years), 17.2\% (aged 15-24 years) [25]. This trend was also reflected among drivers involved in our studio, since the probability of finding a driver under the influence of cannabis is higher the younger the driver.

As for alcohol, it is the most prevalent substance in the in the European DRUID project. In Spain, it is the second most prevalent substance $(6.6 \%)$ after THC, but higher than the European average (3.4\%). Results from previous studies, between $2.1 \%$ and $0.4 \%[5,8,9,23]$, are quite far from those reflected in our study.

It is well known that Spain is the first European country with the highest prevalence of cocaine consumers over the last month $(1.3 \%)$ in the general population (aged 15-64 years) [26] and the second (2\%) in the population aged 15-34 years [10]. Cocaine is the second most consumed illicit drug in Spain after cannabis [26], and the most prevalent illicit substance in those killed in traffic accidents in 2010 [13]. These facts are reflected in our results, since, in the DRUID project, Spain was also the country with the highest prevalence of drivers found positive for cocaine (3.5\%), much higher than the average for those countries participating in the DRUID project $(0.4 \%)$, and even higher than the prevalence of consumption in the last month (1.3\%) and the previous year $(2.7 \%)$ for the general population [26]. It was also higher than that observed in previous roadside studies, which varied between $1.9 \%$ and $0.1 \%[4,7-9]$. This difference between data of general population consumption, and the data found in the general driving population, may be due to the $43.5 \%$ of Spanish drivers are below 40 years [27] and the highest proportion of cocaine users the last month is between 15 and 34 years (2\%) [25].

The benzodiazepines were the most prevalent medicines $(1.6 \%)$, with Spain occupying fourth place in the DRUID project. These figures are lower than those 
obtained in previous studies, $7.2 \%$ [5], 3.7\% [4], 3.6\% [23] and $2.1 \%$ [7]. In our study, as in other previous studies, the benzodiazepines become more prevalent as the drivers' age increases. No Spanish driver was found to be positive for Z-drugs, while they have a relatively high prevalence in the North of Europe $[8,9]$. The hypnotic drug, zolpidem, occupies the third place with regards to consumption of hypnotics in Spain (AEMPS) [28], although one study [29] has indicated that this type of hypnotic drug is mainly used by women aged over 60 , a segment of the population that, in Spain, is a minority as far as driving is concerned.

The data obtained in this study may be surprising, since show that the probability of finding a driver in Spain who has consumed drugs is greater than the probability of driving after having consumed alcohol. We observed a clear difference with respect to the substance consumption data for the Spanish general population (age 15-64) [24], based on self-reference information which point to alcohol as being the most consumed substance in Spain by the general population, and also as the most prevalent substance in most of the European roadside studies [1], as well as in 7 of the 13 countries participating in the DRUID project [16]. Although the general population cannot be equated exactly with the driving population, in Spain almost half (43.5\%) drivers are under 40 years [27] and illicit drugs are consumed in greater proportion by the youngest [25]. Population studies have similarly shown that people frequently to having driven after consuming illicit drugs, in particular cannabis [11].

The increased presence of illegal drugs other than alcohol in the Spanish drivers, could be due to the fact that in Spain, in recent years, measures designed to be a deterrent have been taken with respect to alcohol and driving, including frequent breath test controls and a very strict legislation for those found to be over the limits, so drivers are more highly motivated to avoid driving under the influence of alcohol. Thus, between 2001 and 2010 the number of drivers being found positive in breath tests for alcohol has halved [27]. On the other hand, in Spain, although there is a zero tolerance policy regarding driving under the influence of drugs, drug testing at the roadside has been less frequent than alcohol screening. The driving population is not sensitized to the influence of drugs on driving and information campaigns on legal penalty for driving under 
the influence of illicit drugs or medications have not been very many. Until April 2010, legislation (article 28 of the Reglamento General de Conductores) [15], noted that "drug tests for drivers usually consist of medical examinations and medical tests that medical staff at the closest health center, consider most appropriate". From 2010 [30], officials of the Judicial Police Traffic, have been enabled to carry out saliva drug test for drivers on the road, this will facilitate the progressive implementation of these tests as a daily on Spanish roads.

This study is perhaps the most ambitious carried out in Spain to date on driving and psychoactive substances. A global image is shown for the first time of the incidence of the use of psychoactive substances in the general population of Spanish drivers. Its importance lies in the fact that it allows the results to be extrapolated to the general driving population, because of the sample used, the number of drivers tested, the distribution of the tests in time for determining the substances, and the random nature of the tests. These results will also serve to set the bases of the legal development of roadside drug tests, make public opinion more aware of the problem and support preventive measures in this matter in Spain.

Another of the study's strong points is the obligatory nature of the drivers' participation. Some authors have referred to the fact that a large proportion of drivers, between $1.4 \%$ and $12 \%$ refuse to participate voluntarily in these studies $[7,8]$, and that older drivers are more reluctant to participate voluntarily in roadside studies [23].

The non-response rate of the thirteen prevalence studies within the DRUID project varied markedly between $0 \%$ and $52 \%$ [16]. The possible reasons for non-responses were analysed in detail [16]. One arising issue was the voluntary or mandatory character of the survey. For example in Italy, where participation was mandatory and people who refused cooperation were fined, no participant refused to participate. In Spain, the voluntary participation was requested while drivers were waiting for the result of the mandatory saliva test performed by the police. This also resulted in a very low non-response rate: only 63 out of 2407 drivers did not give their consent to participate. It is obvious that mandatory cooperation resulted in very low non-response rates. 
The representativeness of the studies has been questioned when the refusal rate increases [16]. "Results of the roadside surveys would underestimate the prevalence of psychoactive substances, if drivers under the influence would be more likely to refuse participation. This might for instance be the case if they believed that participation could result in a judicial sanction [16, page 29]" Within the DRUID project it "has been assumed that the effect of non-response bias is small in this roadside survey study when the size of non-response is not exceeding the size of prevalence" [16, page 29].

As limitations, we should point out the police presence at the police check point, which was about 2-3 h, so there may be some bias concerning the prevalence of drivers being found positive with the passage of time, since it has been pointed out that the prevalence of drivers who have drunk alcohol or consumed substances at a police check point decreases over time, probably due to the fact that drivers who pass through a police check point warn other drivers [31,32].

While the prevalence of drivers with positive breath tests for alcohol has been known for some time, the prevalence of other substances has not been known until now. It has been pointed out that roadside studies can be a valuable tool for determining the magnitude and characteristics of the problem of driving under the influence of psychoactive substances. The results indicate that a considerable percentage of drivers had consumed substances that could affect fitness to drive, mainly cannabis and alcohol, and although the consumption of other illicit drugs or medicines is lower, it should not be considered as irrelevant. Our findings suggest that, in Spain, driving after having consumed illicit substances, mainly cannabinoids, could become a bigger problem than driving after consuming alcohol. This requires a response from society comparable to that of recent years to the question of driving under the effects of alcohol. The problem of illicit drugs and medicines in driving should be approached in an integral and multidisciplinary manner, using policies that include specific legislation with legal limits for each substance, strict sanctions which, as in the case of alcohol, have already been seen to be effective, rehabilitation and education plans for offenders, as well as campaigns to inform the public of these policies and of the health risks derived from driving under the effects of certain substances. 


\section{Disclaimers}

(1) This document has been produced under the project "Driving Under the Influence of Drugs, Alcohol and Medicines" (DRUID) financed by the European Community within the framework of the EU 6th Framework Programme (Contract No TREN-05-FP6TR-S07.61320-518404-DRUID).

(2) This document reflects only the authors' view. The European Community is not liable for any use that may be made of the information contained therein.

\section{Competing interests}

The authors declare that they do not have any competing interest.

\section{Acknowledgements}

This study has been possible thanks to the collaboration of the traffic police: The Civil Guard, a traffic police unit of Cataluña and 16 units of local police. We also wish to express our thanks to the staff of the laboratory of the Forensic Toxicology Service, of the University of Santiago de Compostela, and in particular to the following researchers: Óscar Quintela, Marta Concheiro, Ana de Castro and Angelines Cruz, who have contributed in an important manner to the development of the experimental work of this study. The study has been supported by grants from the EU 6th Framework Programme, DRUID project, DGT and the Instituto de Salud Carlos III, Redes Temáticas de Investigación Cooperativa, Red de Trastornos Adictivos RD06/0001/0020. 


\section{References}

1. European Monitoring Centre for Drugs and Drug Addiction (EMCDDA), Annual report 2007. Selected issue on Drugs and Driving, European Monitoring Centre for Drugs and

Drug Addiction, Lisbon, 2007.2 Available http://www.emcdda.europa.eu/attachements.cfm/att_44717_EN_TDSI07003ENC_WEB.pdf

2. Raes, E., Van den Neste, T., Verstraete, A.G., 2008. Drug use, Impaired Driving and Traffic Accidents. European Monitoring Centre for Drugs and Drug Addiction (EMCDDA), Lisbon. (accessed November 2011)

3. Mathijssen, R., Houwing, S., 2005. The prevalence and relative risk of drink and drug driving in the Netherlands: a case-control study in the Tilburg pólice district. Leidschendam: SWOV. http://www.swov.nl/rapport/r-2005-09.pdf (accesses 16.06.14).

4. Dussault, C., Lemire, A.M., Bouchard, J., Brault M., 2000. Drug use among Quebec drivers: the 1999 roadside survey. Proceedings of the 15th International Conference on Alcohol, Drugs and Traffic Safety, Stockholm, Sweden: International Council on Alcohol, Drugs and Traffic Safety, May 22-26.

5. Behrensdorff, I., Steentoft, A., 2003. Medicinal and illegal drugs among Danish car drivers. Accid Anal Prev. 35, 851-860.

6. Drummer, O.H., Gerostamoulos, D., Chu, M., Swann, P., Boorman, M., Cairns, I., 2007. Drugs in oral fluid in randomly selected drivers. Forensic Sci Int. 170, 105-110.

7. Assum, T., Mathijssen, M.P.M., Houwing, S., Buttress, S.C., Sexton, B., Tunbridge, R.J., Oliver, J., 2005. The prevalence of drug driving and relative risk estimations. A study conducted in The Netherlands, Norway and the United Kingdom. IMMORTAL. Deliverable D-R4.2.

8. Gjerde, H., Normann, P.T., Pettersen, B.S., Assum, T., Aldrin, M., Johansen, U., et al., 2008. Prevalence of alcohol and drugs among Norwegian motor vehicle drivers: a roadside survey. Accid Anal Prev. 40, 1765-1772.

9. Gjerde, H., Normann, P.T., Christophersen, A.S., Mørland, J., 2011. Prevalence of driving with blood drug concentrations above proposed new legal limits in Norway: estimations based on drug concentrations in oral fluid. Forensic Sci Int. 210, 221-227.

10. The European Monitoring Centre for Drugs and Drug Addiction (EMCDDA), Annual report on the state of the drugs problem in Europe 2011, European Monitoring Centre for Drugs and Drug Addiction, Lisbon, 2011. 
11. Alvarez, F.J., Fierro, I., Del Rio, M.C., 2007.Cannabis and driving: results from a general population survey. Forensic Sci Int. 170, 111-116.

12. Del Rio, M. C., Gomez, J., Sancho, M., Alvarez, F.J., 2002. Alcohol, illicit drugs and medicinal drugs in fatally injured drivers in Spain between 1991 and 2000. Forensic Sci Int. 127, 63-70.

13. Instituto Nacional de Toxicología y Ciencias Forenses, Memoria análisis toxicológico de muertos en accidente de tráfico (2001-2010), Technical Report, Instituto Nacional de Toxicología y Ciencias Forenses, Ministerio de Justicia, Madrid, 2011.

14. BOE número 288 de 1/12/2007, Ley Orgánica 15/2007, de 30 de noviembre, Capítulo IV, Título XVII, artículos 379.2 y 383, 2007, p. 49505.

15. BOE número 306 de 23/12/2003, Reglamento General de Conductores. Capítulo IV y Capítulo V, artículos 20 a 28, p. 45684.

16. DRUID (Driving Under the Influence of Drugs, Alcohol and Medicines), Prevalence of alcohol and other psychoactive substances in drivers in general traffic. Part I: general results, deliverable D 2.2.3. Part 1, Working Paper, 2011. http://www.druidproject.eu/ (accessed December 2011).

17. Kraemer, C., Pardillo, J., Rocci, S., Romana, M., Ingeniería de Carreteras Volumen II, 8448161106Mc Graw Hill, Interamericana de España S.L. Madrid (2009)

18. Ferrara, S.D., Giorgetti, R., Zancaner, S., 1994. Psychoactive substances and driving: state of the art and methodology. Alcohol and Drugs and Driving, 10, 1-55.

19. Concheiro, M., De Castro, A., Quintela, O., Cruz, A., Lopez-Rivadulla, M., 2008. Determination of illicit and medicinal drugs and their metabolites in oral fluid and preserved oral fluid by liquid chromatography-tandem mass spectrometry. Anal Bioanal Chem. 391, 2329-2338.

20. Gjerde, H., Mordal, J., Christophersen, A.S., Bramness, J.G., Mørland, J., 2010. Comparison of drug concentrations in blood and oral fluid collected with the intercept sampling device. J Anal Toxicol. 34, 204-209.

21. Wille, S.M., Raes, E., Lillsunde, P., Gunnar, T., Laloup, M., Samyn, N., Christophersen, A.S., Moeller, M.R., Hammer, K.P., Verstraete, A.G., 2009. Relationship between oral fluid and blood concentrations of drugs of abuse in drivers suspected of driving under the influence of drugs. Ther. Drug Monit. 31, 511-519.

22. Verstraete, A.G., 2005. Oral fluid testing for driving under the influence of drugs: history, recent progress and remaining challenges. Forensic Sci Int. 150, 143-150. 
22. Krüger, H.P., Schultz, E., Magerl, H., 1995. The German roadside survey 1992-1994. Saliva analyses from an unselected driver population: licit and illicit drugs. C.a.M.A. Kloeden (Ed.), Alcohol, Drugs and Safety-T'95, ANHMRC Road Accident Research Unit, University of Adelaide, Adelaide pp. 55-62

23. Wylie, F.M., Torrance, H., Seymour, A., Buttress, S., Oliver, J.S., 2005. Drugs in oral fluid Part II. Investigation of drugs in drivers. Forensic Sci Int. 150, 199-204.

24. EMCDDA, Statistical bulletin 2011: General population surveys (GPS), Tables. Table GPS-3. Last 30 days prevalence of drug use by age and country, most recent national general population survey available since 2000. GPS Part (ii), Young adults (15-34). GPS Part (iii) Youth (aged 15-24). http://www.emcdda.europa.eu/stats11/gps (accessed January 2012).

25. Delegación de Gobierno para el Plan Nacional sobre Drogas. 2010. Encuesta Domiciliaria sobre Alcohol y Drogas en España EDADES 2009. Madrid: Observatorio Español Sobre Drogas (OESD).

26. Ministerio del Interior. Dirección General de Tráfico. 2011. Las principales cifras de la Siniestralidad vial, España 2010. Madrid: Dirección General de Tráfico.

27. Agencia Española de Medicamentos y Productos Sanitarios (AEMPS). 2011. Uso de benzodiacepinas en España (1992-2006). Ministerio de Sanidad y política Social. Nota informativa de agosto. http://www.aemps.gob.es/medicamentosUsoHumano/observatorio/docs/ansioliticos _hipnoticos.pdf (accessed December 2011)

28. Alejandre, G., Díaz, J., Adan, M.A., Acín, M.T., 2011. The use of hypnotics in primary care. Aten Primaria. 43, 274-275.

29. Boletín Oficial de las Cortes Generales de 28 de abril de 2010. 121/000052, Proyecto de Ley Orgánica por la que se modifica la Ley Orgánica 10/1995 de 23 de Noviembre, del Código Penal. no. 52-11, 2010, p. 47.

30. Vanlaar, W., 2005. Drink driving in Belgium: results from the third and improved roadside survey. Accid. Anal. Prev. 37, 391-397.

31. Vanlaar W., 2008. Less is more: the influence of traffic count on drinking and driving behavior. Accid Anal Prev. 40, 1018-1022. 

Capítulo 3

The Spanish pictogram on medicines and driving: The population's comprehension of and attitudes towards its use on medication packaging

Fierro, I., Gómez-Talegón, T., Álvarez, F.J. Accid Anal Prev, 2013; 50: 1056-1061 



\section{Abstract}

The Spanish pictogram on medicines and driving is legally binding since 2011. We have evaluated patients' comprehension, change in driving frequency and the perceived usefulness, information, comprehensibility, and simplicity of this pictogram on 1385 Spaniards users of the National Health Service (pharmacies, primary care and hospital centres). Most, $85.7 \%$, correctly related the symbol with the possible effects of the medicine on driving and the $83.9 \%$ of the drivers would reduce the frequency with which they drive when prescribed a medicine with such pictogram. The pictogram was found, in a 10-point Likert, useful $(8.3 \pm 1.7)$, informative $(7.7 \pm 1.9)$, comprehensible $(7.8 \pm 1.9)$ and simple $(7.8 \pm 1.9)$. The Spanish pictogram on medicines and driving is understood by the great majority of those interviewed; is well considered by the users of the National Health Service; and offers good prospects for reinforce the awareness of health care professionals and patients on the effects of medicines on driving.

\section{Keywords}

Automobile driving; Drug prescription; Drug utilization; Patient information; Pictograms; Risk assessment 



\section{Introduction}

The use of medicines has been associated with an increase in the risk of involvement in traffic accidents [1,3]. For several years, there have been numerous attempts to classify medications according to the degree of deterioration that they may produce in fitness to drive [4]. In order to warn users of medicines about this possible risk, some countries (recent examples are France and Spain) have opted for printing a pictogram on the medicine's packaging $[5,6]$.

According to the United States Pharmacopoeia [7], pharmaceutical pictograms are "standardized graphic images that help convey medication instructions, precautions, and/or warnings to patients and consumers".

The use of symbols/pictograms allows a message to be expressed in a compact way, which is also quickly identifiable and eliminates language barriers. It would thus seem reasonable to think that the most desirable pictograms would be those with an international validity and which are easily comprehendible for the greatest possible number of people. Both the design and evaluation of pictograms are complex tasks [8], and they are often carried out in several stages that evaluate each modification of the original design. Even though the proliferation of symbols with identical or similar meanings may lead to situations of confusion [9], the presence of pictograms on the labelling of medicines improves the comprehension of the instructions for the use of the medicine [10] and contributes to its acceptance [11].

It is compulsory for pharmaceutical companies in the European Union to provide data concerning the effects of each medication on patients' ability to drive and use machines, prior to commercialisation (Summary of Product Characteristics, Section 4.7) [12]. The insert provided in a medicine's packaging [12] contains information warning of the possible effects patients may notice when driving or handling dangerous machinery, since there is ample evidence that some of the adverse reactions to medications (somnolence, dizziness, blurred vision) may considerably reduce fitness to drive and increasing the risk of involvement in accidents [1-3]. However, not all users of a medicine read the package insert 
$[13,14]$, and many users do not consider the package insert to be very comprehensible [15].

The Royal Decree 1345/2007 regulating the procedure for authorising, registering, and dispensing industrially manufactured medicines for human use was published in Spain in November 2007 [6]. This Decree establishes that newly authorised medicines that may negatively affect fitness to drive, or the ability to handle dangerous machinery, must include a warning symbol (or pictogram) on the packaging. From 2011 onwards, all medicines on sale in Spain that may have an effect on fitness to drive have included a pictogram on the packaging [16]. The said symbol must have the following characteristics (Figure. 1):

(i) A red equilateral triangle with the vertex in the upper part on a white background and a black car inside the red triangle, in the manner of a road traffic sign, and the legend below it, which reads: "Driving: See package insert".

(ii) The size of the Pictogram should be adapted to the size of the package, but in no case should each side of the triangle be shorter than $10 \mathrm{~mm}$. 


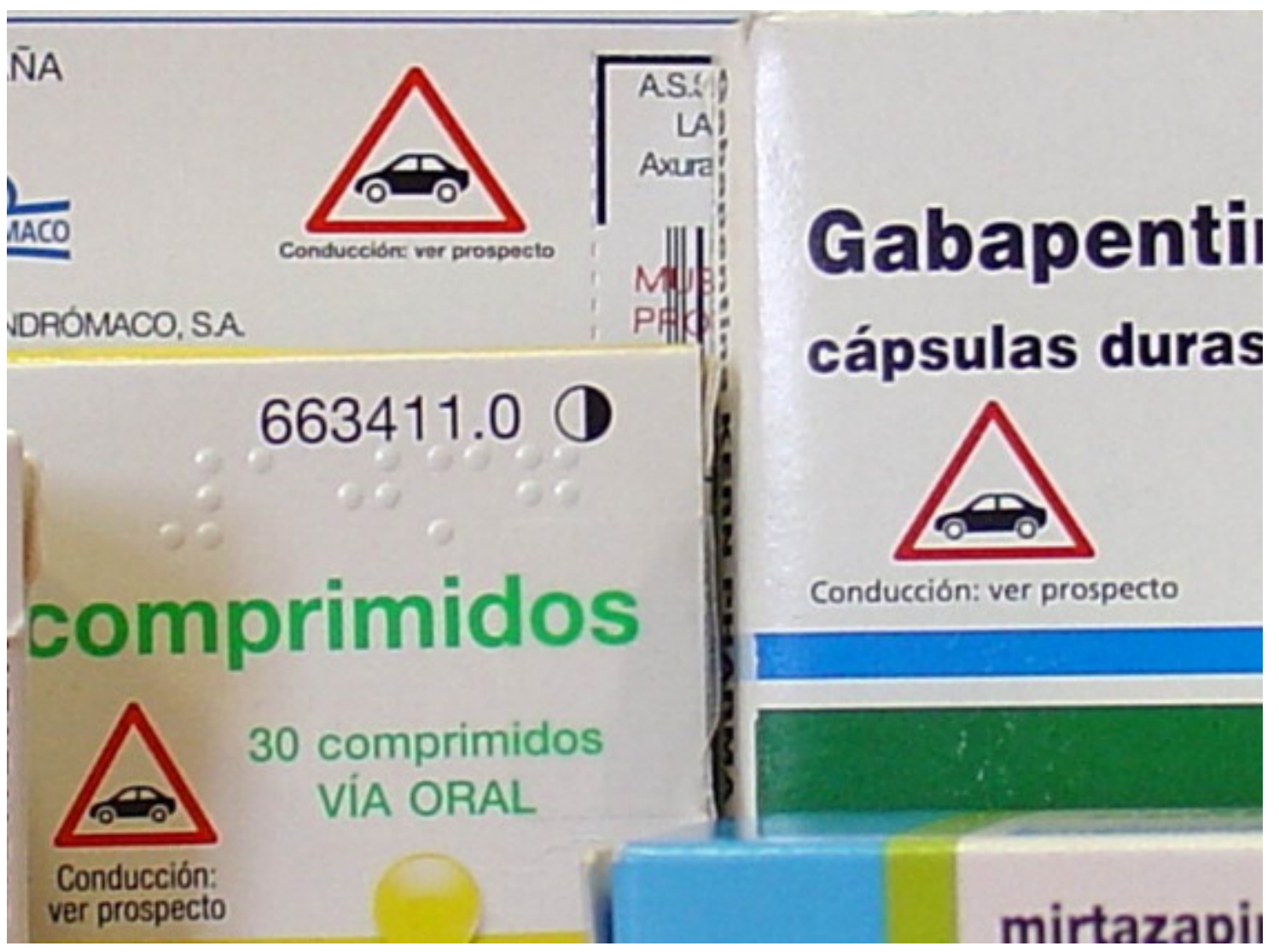

Figure 1 Spanish pictogram on medicines and driving: pictures of some available medicines in the Spanish marked with the legally binding pictogram on medicines and driving.

The aim of the pictogram or symbol is to attract the user's attention so that he/she will read the corresponding patient information insert. The insert found in the medication package [12] already contains a warning about the effects it may have on those who drive or use machinery (section driving and using machines). The aim of this study is to evaluate patients' comprehension of the Spanish pictogram concerning medicines and driving printed on the packaging of certain medications, as well as to analyse the influence this symbol may have on the attitude of the users towards driving (change in driving frequency) and the perceived usefulness, information, comprehensibility, and simplicity of this pictogram. In this study drivers and non-drivers were included as any patient/medicine consumer could find such pictogram in the medicine's packaging. 


\section{Material and methods}

\subsection{Target population}

Non-institutionalized general Spanish population, drivers and non-drivers, aged 18 and over, who reside in Valladolid province (Spain), and are users of the National Health Service (the NHS is a universal with public funding in Spain) when the survey was carried out and, therefore, possible consumers of medicaments in the near future.

\subsection{Sample size and settings}

Questionnaires were filled in through individual randomized interviews among those who attend: (i) Primary Healthcare Centres in 6 surgeries, (ii) preanaesthesia surgery in the Hospital Clínico Universitario and (iii) consumers from five pharmacies. There were 1385 valid interviews made in 2010 from May to October.

This study was approved by the Clinical Research Ethics Committee of the Faculty of Medicine of the University of Valladolid, Reference number 2010/30.

\subsection{Questionnaire}

The following questions were included:

Sociodemographic variables. (i) gender, (ii) age, (iii) driving license, (iv) kilometres driven per year and ( $v$ ) educational level.

Background. In the following item, the interviewees were asked if they knew that some medicines can influence fitness to drive.

Pictogram comprehension. The interviewee was shown the packaging of real medication with a pictogram, acquired in a pharmacy. He/she was then asked, "What do you think this symbol means?" The open answers were grouped into 
five categories, following the model proposed in ISO 9186-1:2007 [17]: $1=$ correct, 2 = wrong, $3=$ wrong and the response given is the opposite of intended meaning, $4=$ the response given is "Don't know", $5=$ no response is given. The percentages were calculated over the total number of replies in the categories 1-4 [17]. The opposite meaning was, in this case, "not to take, or to stop taking, the medicine if you drive".

Estimation of the level of danger of the pictogram on medicines and driving. Then the interviewee was asked: "How would you evaluate the degree of influence of this medicine on driving, i.e., the risk you run using this medicine when driving?" Four options were offered for the answer: "high risk"; "moderate risk"; "low risk"; "no risk" and "I don't know".

Intended change of behaviour - change in driving frequency. "How frequently would you drive during the period in which you were taking the medicine?" The options for answering were: "with the same frequency"; "less frequently"; "a lot less frequently"; "I would hardly drive at all" and "I would not drive at all".

Evaluation of the pictogram. Four aspects of the pictogram shown are scored separately from 1 to 10 (10-point Likert scale): "usefulness" ( $1=$ unnecessary to $10=$ useful $) ; \quad$ "information" $(1=$ not informative to $10=$ informative $) ;$ "comprehensibility" ( $1=$ not understandable to $10=$ comprehensible); and "simplicity" ( $1=$ complex to $10=$ simple). The interviewees were also asked for a global evaluation of the pictogram shown, on a scale of 1 (minimum) to 10 (maximum).

\subsection{Statistical analysis}

For the continuous variables, the mean $\pm S D$ and the frequencies for the category variables are shown. The comparisons between two groups were made through the "Student $t$ " in the case of the continuous variables, and for the categorical variables Pearson's chi-square test was used. 
Logistic regression was used to determine the sociodemographic variables that influence comprehension of the pictogram. The variable with 5 categories (according to ISO 9186-1:2007) [17] was recoded as a dichotomy variable in order to compare the group that answered correctly with the rest. The independent variables introduced into the analysis were: age, gender, educational level, driving license, knowledge that some medicines can influence driving, and prior knowledge of the pictogram.

Logistic regression was also used to evaluate the impact of the pictogram on drivers' attitudes, analysing the possible change in driving frequency. To do so, the 4 categories of the variable that implied a decrease in the frequency of driving were grouped into one, obtaining the dichotomy variable: Does driving frequency decrease si or no. The influence of the above-mentioned factors was controlled for and, in addition, so was the thousands of kilometres driven per year and the different perception of the risk when taking a medicine with the pictogram on its packaging.

A 10-point Likert scale was used to evaluate the pictogram in four different parameters: usefulness, information, comprehensibility, and simplicity. The Cronbach's alpha value was used to prove the reliability of the scale. The influence of the variables, age (ranges, <25; 25-34; 35-44; 45-54; 55-64; >64), gender, educational level, and possessing a driving license, on the scores obtained in the evaluation of these parameters of the pictogram and in the global evaluation were analysed through the use of a non-parametric test (U-MannWhitney or Kruskal-Wallis tests).

A $p$-value $\leq 0.05$ ( $95 \%$ confidence interval) was considered significant. The analyses were carried out using the statistical package PAWS (Predictive Analytics SoftWare, v.18.0 SPSS Inc., Chicago, IL, USA). The level of statistical significance was established at $p \leq 0.05$. 


\section{Results}

A total of 1385 interviews were carried out, $47.7 \%$ were men $(n=660)$ and $52.3 \%$ were women $(n=725)$, with an average age of $50.50 \pm 15.55$ years ( $54.25 \pm 15.70$ years for the men and $47.09 \pm 14.61$ years for the women). Significant differences were observed in the mean ages ( $t=8.758$ [5.553; 8.759]; $p<0.0001)$. 79.3\% had a driving license $(n=1098)$ and $20.7 \%$ did not $(n=287)$.

$93.9 \%$ of those interviewed $(95.4 \%$ of the drivers and $88.5 \%$ of the nondrivers, $\left.X^{2}=18.76 ; p<0.05\right)$ knew that some medicines can influence fitness to drive (Table 1 ).

\subsection{Pictogram comprehension}

Of the 1363 people interviewed who answered the question, "What do you think the symbol means?" $85.7 \%$ (90.5\% of the drivers and $67.4 \%$ of the non-drivers) correctly related the symbol with the possible effects of the medicine on driving. 9.3\% gave a wrong response: $5.1 \%$ stated that they would not take the medicine if they were going to drive, just the meaning opposite to that intended, and $4.2 \%$ gave other answers (Table 2). Significant differences were observed between drivers and non-drivers in the comprehension of the pictogram $\left(X_{3}{ }^{2}=115.24 ; p<0.0001\right)$.

The probability of a correct interpretation decreases in line with the increase in age of those interviewed $\mathrm{OR}=0.969$ [0.957-0.980] and increases in line with the increasing educational level of the interviewees $\mathrm{OR}=1.213$ [1.047-1.405]. These probabilities increase among those who have a driving license OR $=3.268$ [2.315-4.630] and among those who know that some medicines may affect fitness to drive OR $=2.004[1.163-3.448]$. 


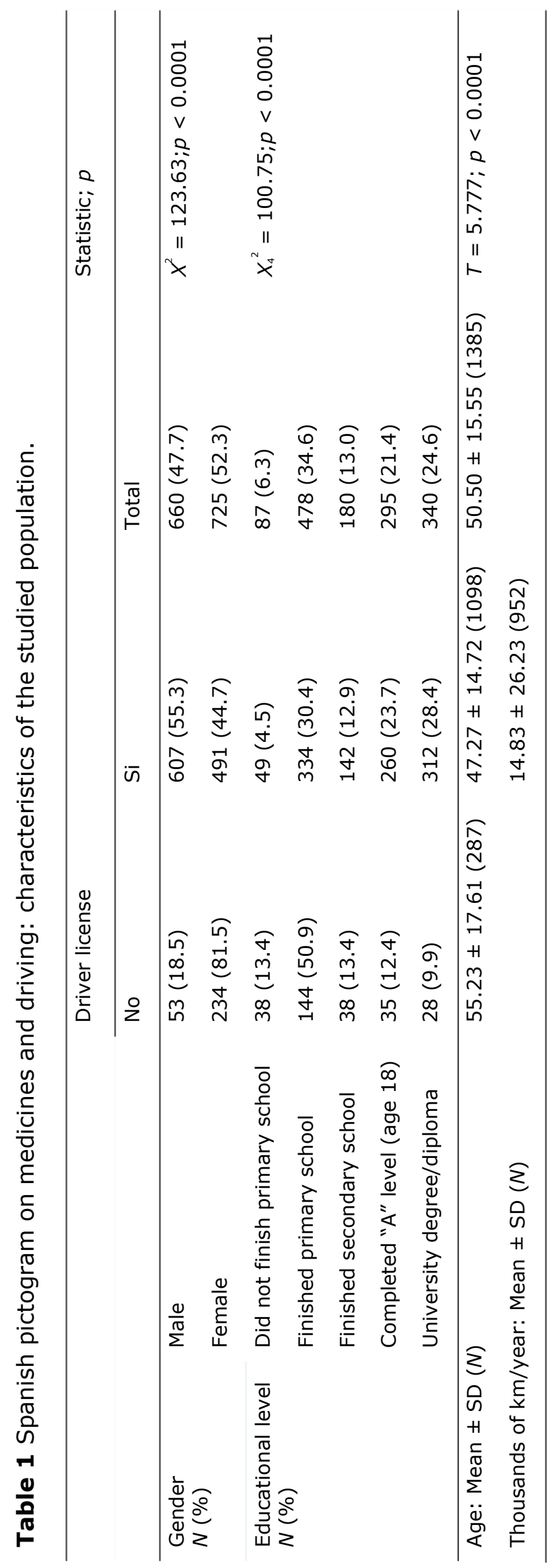




\subsection{Estimation of the level of danger of the pictogram on medicines and driving}

$48.3 \%$ of those interviewed (45.2\% of the drivers and $60.1 \%$ of the non-drivers) believed that the pictogram indicates that there is a "high risk" when driving after taking the medicine with the pictogram on the packaging. 33.9\% referred to a "moderate risk," while $4.5 \%$ referred to a "low risk." Only $0.8 \%$ of the drivers considered that the medicine with the pictogram was "without risk" for the driver. Significant differences were observed between drivers and non-drivers in the perception of risk $\left(X_{4}^{2}=41.77 ; p<0.0001\right.$; Table 2$)$.

\subsection{Intended change of behavior}

Change in driving frequency. As shown in Table 2, only $14.6 \%$ of those interviewed would not reduce their driving frequency if they were prescribed a medicine with a pictogram about driving on the packaging. Differences were observed between the two groups; the drivers being less inclined to change their habits $\left(X_{4}{ }^{2}=41.78 ; p<0.0001\right)$. The decrease in the frequency of driving among drivers is more probable the older the drive is, OR $=1.022$ [1.0081.037], and less probable the more kilometres they normally drove per year, $\mathrm{OR}=0.989$ [0.983-0.994]. Furthermore, taking into consideration those who believed that driving after taking of a medicine with a pictogram "did not imply any risk or that the risk was low", the greater the probability of decrease in the frequency of driving if the attributed risk is "very high", OR $=16.500$ [8.340$32.645]$, or when the risk is considered to be "moderate", OR $=3.557$ [1.923$6.577]$. 


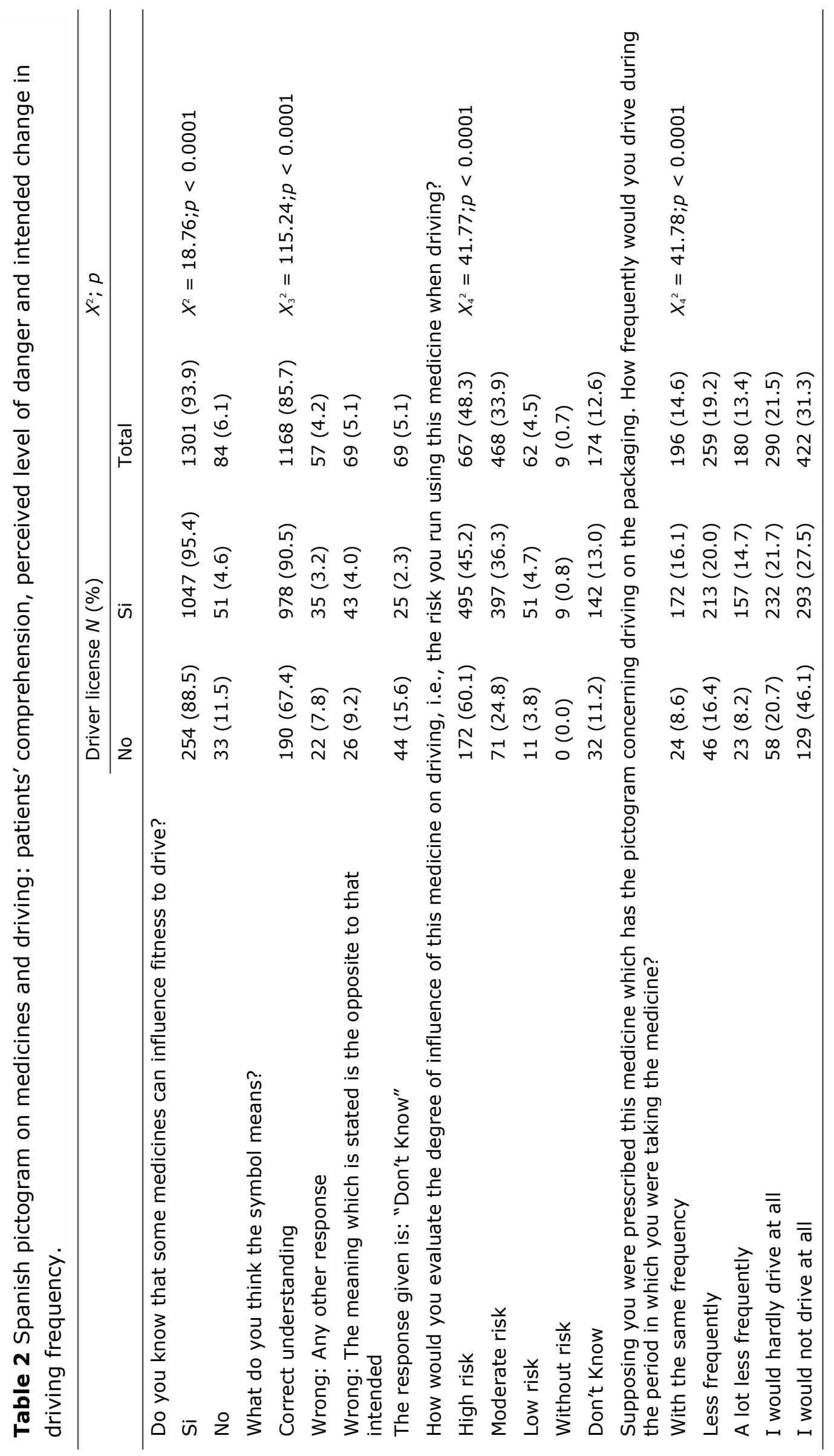




\subsection{Evaluation of the pictogram}

The pictogram was found to be useful, informative, comprehensible and simple. Table 3 shows the average scores (Mean \pm SD) given to different parameters of the pictogram (usefulness, information, comprehensibility and simplicity). The Cronbach's alpha value of 0.837 proves the reliability of the $10-$ point Likert scale to evaluate these parameters of the pictogram.

The influence of the variables age, gender, educational level, and possessing a driving license on the scores obtained in the evaluation of usefulness, information, comprehensibility, and simplicity was analysed using non-parametric tests (Table 3). Gender had no influence on the scores. Significant differences were observed in the mean score given to the different aspects of the pictogram according to the age range of those interviewed: the highest means were observed for all the scores in those interviewees aged 25-34 and 35-44, while the lowest means were observed in those aged over 64. In general, the higher the education level, the higher the scores of all the parameters of the pictogram. The drivers gave higher scores than the non-drivers to the comprehensibility and simplicity of the pictogram (Table 3 ).

As for the Global Evaluation of the pictogram, the average score obtained was $7.98 \pm 1.58$ points. The global score given to the Spanish pictogram is independent of: the age of those interviewed, their gender, their educational level, and whether or not they possessed a driving license (Table 3). 


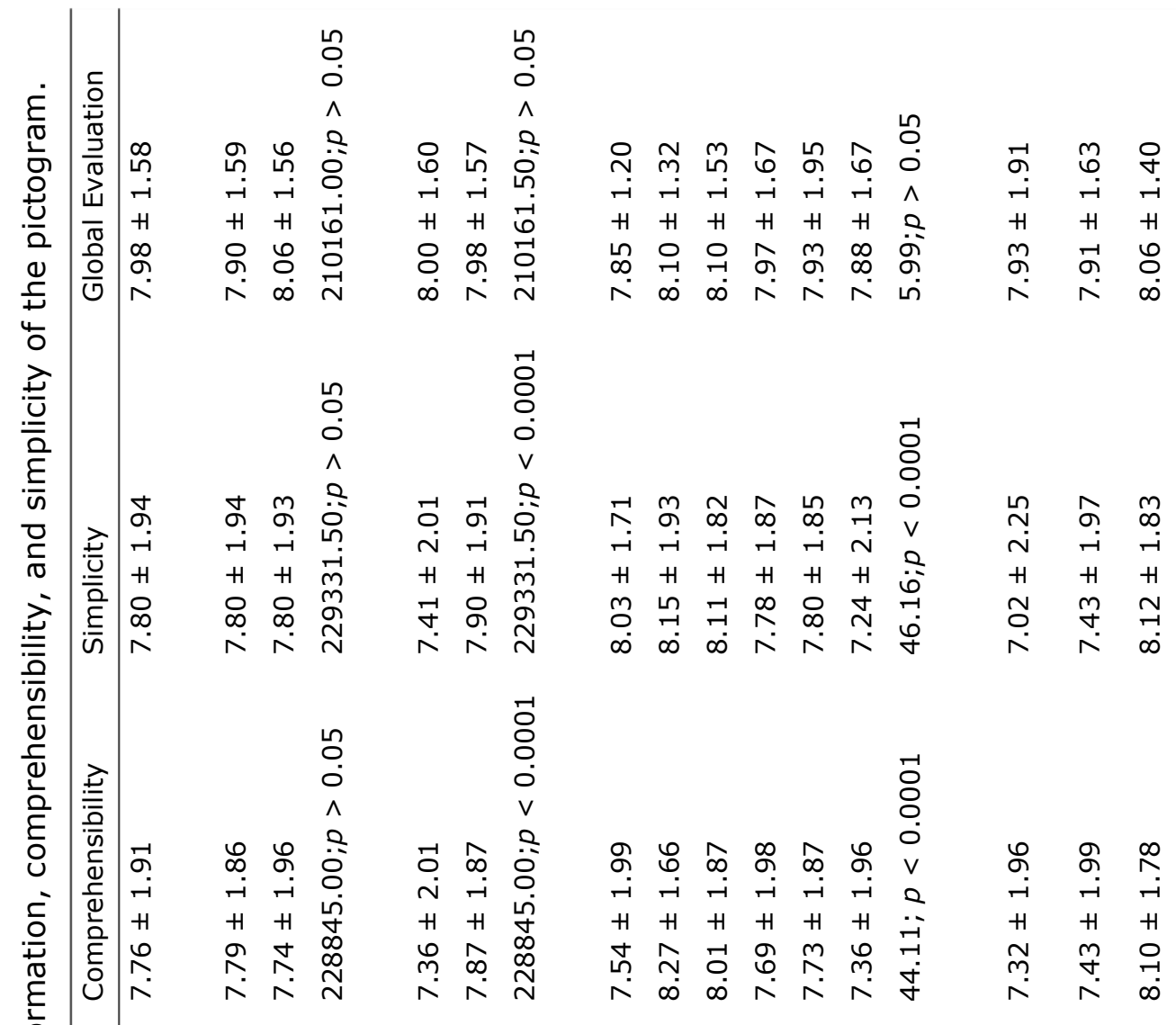

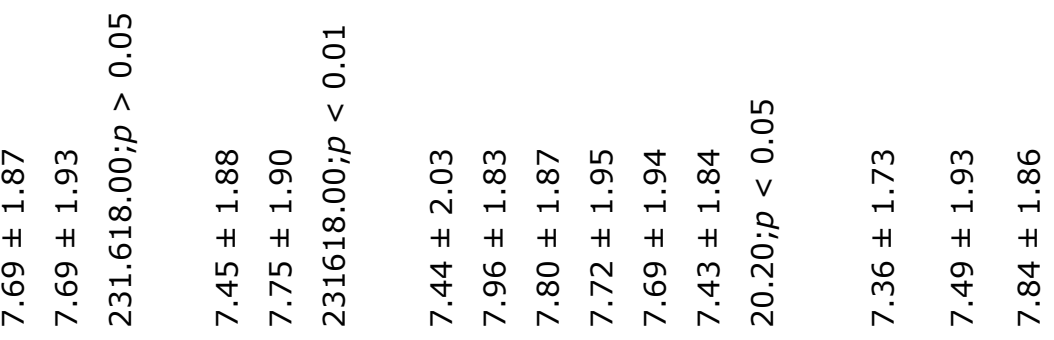

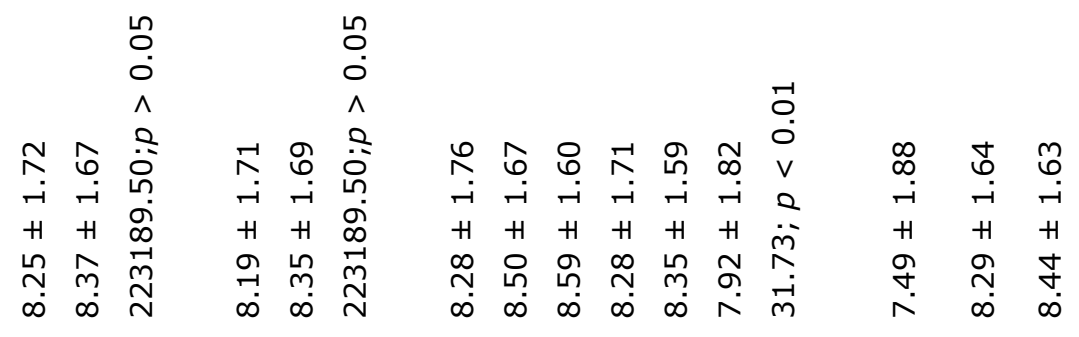

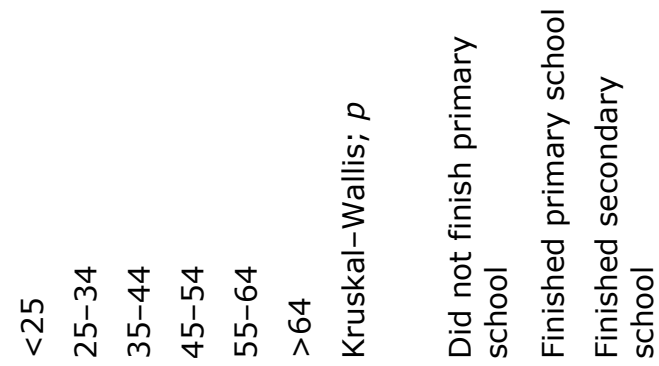

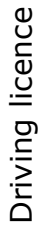




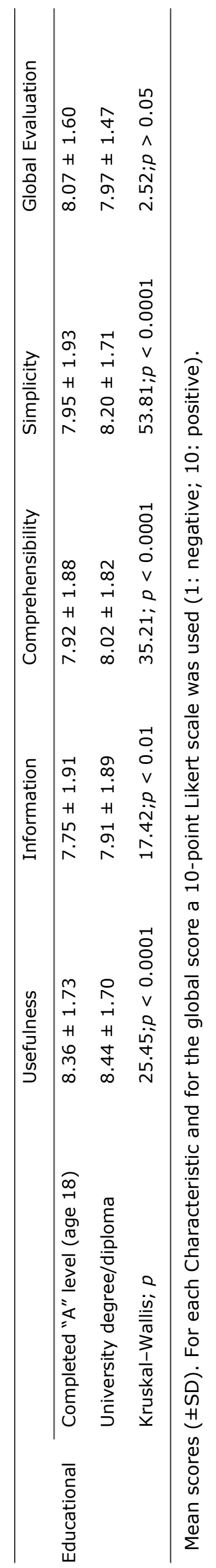




\section{Discussion}

The results of the study show that the Spanish pictogram on medicines and driving is, in general, well valued by the users of medicines and its meaning is comprehended by a high percentage of those interviewed. This is especially true among those who possess a driving license, a circumstance that is favourable as this question mainly affects drivers. The better comprehension of the pictogram by drivers may mostly be due to the design type of the pictogram, which is closely related to that of traffic signs. Although in previous studies, it has also been observed that driving experience improves a person's capacity to interpret other types of signs, such as, for instance, those related with industrial safety [18].

On the other hand, it must be said that when the interviews were held (May to October 2010) few medicines had this pictogram on the packaging, and, therefore, it had not been widely seen among the population. This offers a good perspective to the symbol's usefulness, since, as observed in various studies, familiarity with the signs improves comprehension $[9,19]$. Therefore, it is reasonable to expect that the comprehension of this symbol among the population will increase as it becomes more widely used and is included in publicity campaigns.

Our study reveals that the probability of correctly interpreting this pictogram decreases as the age of those interviewed increases, and it increases if the interviewee has a higher the educational level. It also increases if those interviewed had prior knowledge of medicines that can influence fitness to drive. The influence of age in the comprehension of different pictograms has already been analysed in several studies [9]. Hancock et al. [20] observed that age affects the comprehension of the warning messages. They compared a group of young adults (18-22 years of age) with another group of elderly adults (64-76 years of age). They observed that elderly adults' capacity to deduce information from the warning messages was inferior to that of the young adults. Easterby and Hakiel [19], analysing several groups of consumers, observed that consumers over the age 55 had a lower comprehension of the symbols on the labels of different products. 
There are also numerous studies showing that comprehension of graphic messages and, in particular, comprehension of warning labels on medicines improves in line with the educational level of the interviewee [21,22]. However, it is precisely those with a lower educational level who would most benefit from the use of pictograms [23]. Pictograms can be of great help in the acceptance of medication and in the comprehension of the use of a prescription for those patients with lower levels of education, even for those unable to read and write $[11,22,24]$.

In our case, and in view of the results, the presence of the pictogram on medication packaging may play a fundamental role in changing drivers' attitudes (83.9\% of the drivers would reduce their driving frequency). The same was not observed in a Dutch study carried out with drivers suffering from chronic pain. In this case, the authors concluded that the warning labels did not significantly modify the attitude towards driving of patients who were taking medicines with a psychotropic effect [25].

It has been observed that a greater perception of risk improved the disposition to read warning messages $[26,27]$, although the final decision could be influenced more by the person's own perception of the risk than by the warning message [9]. Davies et al. [22] demonstrate the influence of the perception of risk when they observed that parents' final decisions on whether to purchase or reject a toy were influenced more by the perception of the product's danger than by the warning message on the packaging. The influence of the attributed risk of the pictogram, which was also shown in this study, is an important variable when considering a person's willingness to change their attitude towards driving. This factor deserves to be taken into account given that the interpretation of the risk in the Spanish pictogram could be highly subjective. This holds true for all medicines in regards to informing patients about the effects that a medication may have on a patient's fitness to drive.

Our study had several potential limitations. This study was conceived as an exploratory survey to evaluate the comprehensibility of the pictogram on medicines and driving, which is why it was done in a limited geographical area. Therefore, the results should not be extrapolated to the national level. 
Furthermore, at the time the surveys were being carried out, only the packaging of medicines for the central nervous system had been revised to include the pictogram. This may have had an influence on some of the results, especially in the estimation of the level of danger. In this study, we have tried to provide an adequate context in which to show the symbol, since, as some studies have shown, comprehension of a symbol depends on the context [28]. However, this means that the symbol's capacity to attract attention cannot be evaluated since the medicine's packaging was shown to the interviewee as encouragement to observe the pictogram when conducting the survey. This fact could have diminished the symbol's real effectiveness of reducing the number of accidents because if it had not been noticed, it would not have had an effect at all on the driver's attitude. On the other hand, since the symbol is already being used on medication packaging, certain factors such as the influence of the size [29] the setting, the design [30] etc., on the interpretation of the pictogram have not been analysed.

\section{Conclusion}

The Spanish pictogram on medicines and driving is understood by the great majority of the interviewees, is well valued by users of the National Health Service. This pictogram can be seen as a tool to improve prescribing and dispensing procedures of medicines that impair driving as well as an instrument to make patients aware of the role of medicines play in traffic safety.

\section{Conflict of interest}

The authors declared no conflict of interest.

\section{Acknowledgements}

The study was suported by grants from the Consejería de Sanidad, Junta de Castilla y León (ORDEN SAN/1778/2009) and the Agencia Española de 
Medicamentos y Productos Sanitarios, Ministerio de Sanidad y Política Social (Resolución de 22 de marzo de 2010).

The authors of the Spanish study would like to thank the patients and health professionals (physicians, pharmacists and nursing staff) involved in the study for their participation. We would also like to thank the health authorities (Junta de Castilla y León, Consejería de Sanidad, Sacyl, Ministerio de Sanidad y Consumo-Agencia Española de Medicamentos y Productos Sanitarios (AEMPS)), the Primary Health Care Centers, the Hospital Clínico Universitario of Valladolid, the Colegio Oficial de Farmacéuticos de Valladolid, SEMT, and SET for their collaboration at all times. Special thanks to Lurdes Rico for their contribution to the study. 


\section{References}

1. Orriols, L., Salmi, L.R., Philip, P., Moore, N., Delorme, B., Castot, A., Lagarde, E., 2009. The impact of medicinal drugs on traffic safety: a systematic review of epidemiological studies. Pharmacoepidemiology and Drug Safety 18, 647-658.

2. Orriols, L., Delorme, B., Gadegbeku, B., Tricotel, A., Contrand, B., Laumon, B., Salmi, L.R., Lagarde, E., CESIR research group, 2010. Prescription medicines and the risk of road traffic crashes: a French registry-based study. PLoS Medicine 7,e1000366.

3. Engeland, A., Skurtveit, S., Morland, J., 2007. Risk of road traffic accidents associated with the prescription of drugs: a registry-based cohort study. Annals of Epidemiology $17,597-602$.

4. Ravera, S., Monteiro, S.P., de Gier, J.J., van der Linden, T., Gomez-Talegon, T., Alvarez, F.J., the DRUID Project WP4 Partners, 2012. A European approach to categorising medicines for fitness to drive: outcomes of the DRUID project. British Journal of Clinical Pharmacology (March), http://dx.doi.org/10.1111/j. 13652125.2012.04279.x Epub ahead of printPubMed PMID:22452358.

5. Ministère de la Santé et des Solidarites, 2005. Arrete du 18 Juillet 2005 pris pour I'application de l'article R. 5121-139 du code de la sante publique et relative a I'opposition d'un pictogramme sur le conditionnement exterieur de certain medicaments et produits. Journal Officiel de la Republique FranÇaise, 2 Août 2005 (SAN/P0522726A).

6. Ministerio de Sanidad y Consumo, 2007. REAL DECRETO 1345/2007, de 11 de octubre, por el que se regula el procedimiento de autorización, registro y condiciones de dispensación de los medicamentos de uso humano fabricados industrialmente. BOE $267,45652-45698$.

7. United States Pharmacopoeia, 2011.

http://www.usp.org/audiences/consumers/pictograms/ (December 2014).

8. Wogalter, M.S., Conzola, V.C., Smith-Jackson, T.L., 2002. Research-based guidelines for warning design and evaluation. Applied Ergonomics 33, 219-230.

9. Davies, S., Haines, H., Norris, B., Wilson, J.R., 1998. Safety pictograms: are they getting the message across? Applied Ergonomics 29, 15-23.

10. Houts, P.S., Doak, C.C., Doak, L.G., Loscalzo, M.J., 2006. The role of pictures in improving health communication. A review of research on attention, comprehension, recall, and adherence. Patient Education and Counseling 61, 173-190. 
11. Dowse, R., Ehlers, M., 2005. Medicine labels incorporating pictograms: do they influence understanding and adherence? Patient Education and Counseling 58, 6370.

12. European Council Directive 83/570/EEC of 26 October 1983 on the approximation of provisions laid down by law, regulation or administrative action relating to proprietary medicinal products, $1983 . \quad$ http://eurlex.europa.eu/LexUriServ/LexUriServ.do?uri=CELEX:31983L0570:EN:HTML (January 2012).

13. Bernardini, C., Ambrogi, V., Perioli, L.C., Tiralti, M.C., Fardella, G., 2000. Comprehensibility of the package leaflets of all medicinal products for human use: a questionnaire survey about the use of symbols and pictograms. Pharmacological Research 41, 679-688.

14. Nathan, J.P., Zerilli, T., Cicero, L.A., Rosenberg, J.M., 2007. Patients' use and perception of medication information leaflets. Annals of Pharmacotherapy 41, 777782.

15. Barrio-Cantalejo, I.M., Simon-Lorda, P., March Cerda, J.C., Prieto Rodriguez, M.A., 2008. Grammatical readability of the package leaflets of the medicinal products most widely consumed and generating the highest expense in Spain during 2005. Revista Española de Salud Publica 82, 559-566.

16. Agencia Española de Medicamentos y Productos Sanitarios, 2012. Medicamentos y Conducción http://www.aemps.gob.es/industria/etiquetado/conduccion/home.htm (January 2012).

17. ISO 9186-1:2007, 2007. Graphical Symbols - Test Methods - Part 1: Methods for Testing Comprehensibility. ISO 9186-1.

18. Chan, A.H.S., Ng, A.W.Y., 2010. Investigation of guessability of industrial safety signs: effects of prospective-user factors and cognitive sign features. International Journal of Industrial Ergonomics 40, 689-697.

19. Easterby, R.S., Hakiel, S.R., 1981. Field testing of consumer safety signs: the comprehension of pictorially presented messages. Applied Ergonomics 12, 143-152.

20. Hancock, H.E., Fisk, A.D., Rogers, W.A., 2005. Comprehending product warning information: age-related effects and the roles of memory, inferencing, and knowledge. Human Factors 47, 219-234.

21. Dowse, R., Ehlers, M.S., 2003. The influence of education on the interpretation of pharmaceutical pictograms for communicating medicine instructions. International Journal of Pharmacy Practice 11, 11-18. 
22. Davis, T.C., Wolf, M.S., Bass, P.F., Middlebrooks, M., Kennen, E., Baker, D.W., Bennett, C.L., Durazo-Arvizu, R., Bocchini, A., Savory, S., Parker, R.M., 2006. Low literacy impairs comprehension of prescription drug warning labels. Journal of General Internal Medicine 21, 847-851.

23. Michielutte, R., Bahnson, J., Dignan, M.B., Schroeder, E.M., 1992. The use of illustrations and narrative text style to improve readability of a health education brochure. Journal of Cancer Education 7, 251-260.

24. Dowse, R., Ehlers, M.S., 2001. The evaluation of pharmaceutical pictograms in a lowliterate South African population. Patient Education and Counseling 45, 87-99.

25. Veldhuijzen, D.S., van Wijck, A.J., Verster, J.C., Kalkman, C.J., Kenemans, J.L., Olivier, B., Volkerts, E.R., 2006. The impact of chronic pain patients' psychotropic drug knowledge and warning labels on the decision whether to drive a car or not. Traffic Injury Prevention 7, 360-364.

26. Wogalter, M.S., Brelsford, J.W., Desaulniers, D.R., Laughery, K.R., 1991. Consumer product warnings: the role of hazard perception. Journal of Safety Research 22, 7182.

27. Wogalter, M.S., Brems, D.J., Martin, E.G., 1993. Risk perception of common consumer products: judgments of accident frequency and precautionary intent. Journal of Safety Research 24, 97-106.

28. Lehto, M.R., 2000. Designing warning signs and warning labels: part II - scientific basis for initial guidelines. In: Anil Mital, Asa Kilbom, Shrawan Kumar (Eds.), Elsevier Ergonomics Book Series. , pp. 257-280.

29. Shieh, K., Huang, S., 2003. Factors affecting preference ratings of prohibitive symbols. Applied Ergonomics 34, 581-587.

30. ISO 3864-2:2004, 2004. Graphical Symbols - Safety Colours and Safety Signs Part 2: Design Principles for Product Safety Labels. ISO 3864-2. 
Capítulo 4

The relationship between observed signs of impairment and THC concentration in oral fluid

Fierro, I., González-Luque, T., Álvarez, J.C.

Drug Alcohol Depend, 2014; 144: 231-238 



\section{Abstract}

Background: Studies have shown that cannabis intake increases the risk of traffic accidents. Controlled experiments support these findings and have shown a positive dose-effect relationship.

Methods: In this retrospective cross-sectional study of data from a roadside survey we investigated whether a police officer's judgment regarding signs of impairment is related to the concentration of delta-9-tetrahydrocannabinol (THC) in the oral fluid (OF). We investigated 2632 cases from a representative sample of 3302 Spanish drivers: 253 drivers positive for THC only, 32 positive for THC and ethanol, 201 with only ethanol detected in their breath, and 2146 drivers who tested negative for ethanol in breath and drugs in OF. Recorded data comprised breath alcohol concentrations, THC concentrations in the OF, and the 31 observed signs of impairment. Subject groups were compared using the chisquare test, and logistic regression was used to examine the risk of being categorized as exhibiting signs of impairment.

Results: A relationship was found between the OF THC concentration and some observed signs of impairment. Eye signs were noticeable from a THC concentration $>3.0 \mathrm{ng} / \mathrm{ml}$ in $\mathrm{OF}$, and $>25 \mathrm{ng} / \mathrm{ml}$ was related to behavior, facial expression, and speech signs. Alcohol and THC contribute to impairment independently and, when taken simultaneously, the effects are comparable to the sum of the effects when consumed separately.

Conclusions: The observation of signs of impairment due to cannabis occurs in an OF concentration-related manner but, as a clinical test, OF has low sensitivity and specificity in a random roadside survey.

\section{Keywords}

Cannabinoids; diagnostic test; automobile driver examination; psychomotor impairment; traffic accidents. 



\section{Introduction}

Alcohol and drugs impair a person's ability to concentrate and make decisions and slow reaction time to the roadway environment [1]. Alcohol and cannabis are the substances most frequently used by drivers [2], and both are frequently detected in injured and killed drivers [3]. Growing scientific evidence links increased traffic accident risk and driving under the effects of cannabis [4,5], and a dose-effect relationship has been reported $[6,7]$. The increased risk is greater if cannabis is used in conjunction with alcohol and/or other illicit drugs.

Countries have different laws concerning driving under the influence (DUI) of drugs. Drug per se laws, analogous to zero-tolerance laws, make it illegal for a driver to have certain drugs in their system. In other cases, observed signs of impairment and the evidence of drugs in a laboratory test are used to bring charges of drug-impaired driving. In some countries it is illegal to drive under the influence of drugs and the presence of signs of impairment can result in more severe sentencing for driving offenses.

In the impairment approach, driver fitness is observed and assessed, penalizing those who are actually impaired. However, a lack of standardized methods for measuring and judging driver impairment makes the assessment somewhat arbitrary; legal disputes are common, making enforcement of these laws costly [8]. In some countries the per se limits have been set at the limit of detection and are de facto zero limits. According to Grotenhermen et al. [8], "This avoids the need for a reliable science-based correlation between drug concentration and level of impairment and facilitates enforcement. However, zero limits by design penalize the presence in body fluids of an active drug ingredient or its metabolites, which does not necessarily correspond to actual impairment."

Standardized field sobriety tests (SFSTs) are used as qualitative indicators of impairment in individuals suspected of DUI [9]. SFSTs have been demonstrated to be sensitive to impairment with relation to blood alcohol concentrations (BACs) above and below $0.08 \%[10,11]$. Nevertheless, similar studies have shown that SFSTs are poor at detecting delta-9-tetrahydrocannabinol (THC) impairment. However, various countries use these types of performance-based 
tests to detect impairment associated with drugs other than alcohol [12]. In order to increase the sensitivity of SFSTs for THC, the addition of some tests and observations has been suggested, such as "head movements or jerks" $[13,14]$ or a series of observations conducted by the physician as part of the Norwegian clinical test of impairment [15]. Even with these extra observations, however, THC impairment is difficult to detect, particularly at lower THC concentrations $[16]$.

Blood is still the gold standard for determining drug impairment in DUI cases [17], but the use of oral fluid (OF) has increased over the last decade, leading to the need for a rapid, simple, and reliable on-site Of testing device [18]. The main benefit of such a sample is its non-invasive collection compared to blood and the inconvenience/embarrassment of obtaining urine. Moreover, OF sampling seems to be better accepted by people than urine or blood sampling, and samples can be taken by the police officers themselves, simplifying the enforcement procedure $[19,20]$. However, OF is not yet the best matrix for the detection and confirmation of the presence of drugs in drivers, and most studies showing that cannabis impairs driving performance in a concentration-related manner use blood as the matrix $[6,7,15]$.

Because OF is a suitable matrix for drug screening purposes, an attempt has been made to establish fixed ratios or conversion factors between the drug concentrations in blood and OF. The large individual variations do not generally allow applicable OF/blood ratios for most drugs, so drug concentrations in OF may not accurately estimate individual drug concentrations in blood [17,21-23]. However, in epidemiological research, drug concentrations in OF can provide valuable information. Recent studies show the possibility of estimating the prevalence of blood drug concentrations above chosen limits in a population by analyzing OF [24]. In addition, equivalent cut-off thresholds can be estimated for drugs (THC and amphetamine) in blood and OF from a selected population using a regression model with concentration percentiles in OF as the response variable and the corresponding concentration percentiles in blood as the predictor variable [20]. The regression model opens up new possibilities and can be used to obtain valuable additional information on possible drug impairment in roadside surveys of drugs and driving. 
Ramaekers et al. [25] previously found a low correlation between the magnitude of change in performance impairment and THC in OF and serum, but they found a promising method of defining threshold levels for impairment by comparing the proportion of observed impairment or no impairment as a function of the THC concentration in blood or OF. Recently, a Norwegian study confirmed a positive concentration-effect relationship between blood THC concentration and impairment in a group of apprehended drivers [15]. Taking these results into account, as well as the population hypothesis of Gjerde \& Verstraete [20], our hypothesis is that a positive concentration-effect relationship exists between the THC concentration in OF and the percentage of drivers observed with signs of impairment in a random roadside survey.

The aims of the present study were to:

- Analyze the percentages of drivers with signs of impairment in each substance group (alcohol, THC, and alcohol + THC).

- Investigate which signs of the clinical test are potentially relevant for assessing DUI of THC.

- Investigate the possible concentration-effect relationship between THC concentration in OF and impairment observed in drivers.

- Analyze the contribution of alcohol and THC, both together and separately, to the probability of observing signs of impairment.

\section{Material and methods}

\subsection{Sample and assessment procedures}

The data were taken from the Spanish database generated for the roadside survey in the Driving Under the Influence of Drugs, Alcohol, and Medicines (DRUID) project, a cross-sectional study with stratified random sampling. The strata were country area, road type, and period of time. In Spain, the survey was carried out during the period from July 2008 through August 2009 [26]. The study was approved by the Research Ethics Committee of the University of Valladolid on January 31, 2007. 
For the roadside survey, the subjects were selected at random. The police

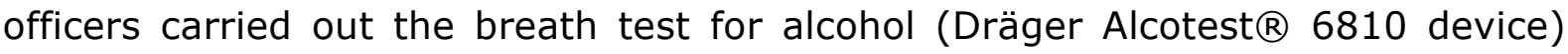
and an on-site OF test for drugs (Dräger Drug Test ${ }^{\circledR}$ 5000). Both tests were mandatory for all controlled drivers, but participation in the study was voluntary. A short interview was performed in which the police officer asked the driver questions about drinking habits and drug history, as well as recent use. Data collected for participants were anonymous and included date, time, gender, age, vehicle and road type, clinical signs of impairment (31 external signs of drug consumption), self-reported drug use, and the reason of refusal for those who refused. Voluntary participation was requested and all participants provided informed consent. Oral fluid samples were taken for confirmatory analysis while drivers were waiting for the result of the mandatory on-site OF test. The sequence of police enforcement and research activities resulted in very low nonresponse rates $(1.8 \%)$ [2].

The police officers were formally trained for observing signs of impairment, including 20 hours of theoretical and practice training by the University of Valladolid and Directorate General for Traffic. The practice included a real roadside survey. The presence or absence of 31 signs of impairment were observed in drivers (Table 1), including seven attitude signs, four body appearance signs, five facial expression signs, three speech signs, four coordination signs, and eight eye signs. At least two officers were present when signs were observed. The checklist was completed by the police officer. A similar test developed by the Institute for Road Safety Research in the Netherlands (SWOV) was previously used in the IMMORTAL project [27], but some signs have been added. The checklist was based on several existing checklists, including one developed for the German police [28], and supplemented with two questions about recent drug use. These supplementary questions have not been taken into account for this article.

The OF samples for confirmatory analysis were collected with the StatSureTM Saliva•SamplerTM (Statsure Diagnostic Systems, Framingham, MA, USA), an OF collector with good recovery and stability of drugs in the OF during storage. The test tubes were sent to the participating laboratory, refrigerated at all times (between $2^{\circ} \mathrm{C}$ and $8^{\circ} \mathrm{C}$, or frozen if transport time was $>24$ hours) using devices 
with frozen gel (thermovials Kern(C) and isothermal boxes. All samples were analyzed within 36 hours after being collected. Oral fluid samples were screened and subsequently confirmed by LC-MS/MS quantification as described by Concheiro et al. [29]. As part of the protocol of the DRUID project, 22 substances (drugs and some metabolites) were screened in each OF sample: amphetamine, MDMA, MDA, MDEA, methamphetamine, cocaine, benzoylecgonine, THC, THC-COOH, 6-acetylmorphine, morphine, codeine, methadone, hypnotics and sedatives (zolpidem, zopiclone, flunitrazepam), and anxiolytics (alprazolam, clonazepam, diazepam, lorazepam, nordiazepam, oxazepam). In addition, amitriptyline, diphenhydramine, and levo-promazine were analyzed in Spain [30].

We investigated 2632 cases from a representative sample of 3302 Spanish drivers. The sample included 253 cases positive for THC only and 32 cases positive for THC and ethanol but not any other drug. These cases were compared to 201 drivers positive for only ethanol and 2146 drivers negative for drugs in their OF and alcohol in breath air. In the rest of the drivers $(n=670)$, substances other than alcohol or THC were detected $(n=639)$ or no data on the presence or absence of signs were recorded $(n=31)$, and all of these drivers were excluded from the study. The detection limits were $1 \mathrm{ng}$ THC per $\mathrm{ml}$ of OF and $0.01 \mathrm{mg}$ of ethanol per liter of breath air.

The group under the influence of only alcohol was compared to the groups under the influence of THC (THC alone and alcohol+THC) to prove the reliability of the signs test for determining impairment $[15,31]$. According to the Spanish legal limits for DUI of alcohol and many European countries [32], two alcohol concentration levels were considered (0.01-0.25 mg/l and $>0.25 \mathrm{mg} / \mathrm{l})$.

\subsection{Analysis}

The sensitivity, specificity, and accuracy of the selected on-site screening device (Dräger Drug Test $\circledast 5000$ ) and observational test were assessed by comparing both results with the OF confirmation analysis in the laboratory. 
The cut-off for THC (27 ng of delta-9-THC per $\mathrm{ml}$ of OF) was proposed in the DRUID project [26] based on the lowest limit of quantitation (LOQ) that could be measured by all toxicological laboratories involved in the analysis of the substances in the project, regardless of the matrix analyzed (blood, serum, or OF). For THC, the LOQ was established in $1 \mathrm{ng} / \mathrm{ml}$, but a person usually has a higher concentration in OF than in blood. The equivalent cut-off for THC in OF was $27 \mathrm{ng} / \mathrm{ml}$ to obtain the same prevalence when blood or OF matrix were used $[17,22]$. In the case of alcohol, the sensitivity, specificity, and accuracy of the observational test (for total signs of impairment and different groups of signs) were compared to the results of the breath air test (Dräger Alcotest $\mathbb{R} 6810$ device), and the cut-off $(0.25 \mathrm{mg} / \mathrm{l})$ was the Spanish legal limit for drivers of private cars. Criteria for impaired drivers were one or more of the listed signs.

In order to investigate a possible relationship between the THC concentration in OF and observed signs (total signs, groups of signs, or an individual sign), we did not presuppose concentration intervals for THC in OF for impairment. We considered the OF concentration of THC as a possible predictive factor for the presence of signs, whereas changes in sensitivity and specificity for the presence of any sign determined the concentration intervals. The concentration groupings for THC in OF were based on cut-offs in a ROC analysis carried out with the THC alone group. We excluded 45 cases from the ROC analysis because they had THC concentrations $>200 \mathrm{ng} / \mathrm{ml}$, but without specifying the exact concentration. These cases were considered in the other analysis and were included in the upper range of concentration ( $>100 \mathrm{ng} / \mathrm{ml}$ ). We determined four intervals for the concentration of THC in OF: $\leq 3.00 \mathrm{ng} / \mathrm{ml} ; 3.01-25.00 \mathrm{ng} / \mathrm{ml} ; 25.01-100.00$ $\mathrm{ng} / \mathrm{ml}$; and $>100 \mathrm{ng} / \mathrm{ml}$ ).

Pearson's chi-squared test was used for categorical data when comparing differences between groups; test for associations between the concentration ranges and observed signs of impairment; and, using the alcohol group, to prove the validity of the test for determining impairment due to substances.

Controlling for the potential confounding effects of sociodemographic factors (age, gender) and stratification factors (country zone, type of road, and time period), logistic regression (step forward) was used to examine the relative risk 
of being categorized as having signs of impairment when THC and/or alcohol were detected at different concentrations. Interaction effects between drug and alcohol were considered. Other possible interactions between alcohol or cannabis and the rest of the categorical variables were analyzed in the logistic model (THC*gender; THC* country zone; THC*type of road; THC*time period, and the same interactions for alcohol concentration ranges).

Statistical analyses were performed using IBM SPSS Statistics 19. Significance was established at $p \leq 0.05$.

\section{Results}

\subsection{Reliability of the clinical sign test}

The reliability of the test was evaluated using the groups of alcohol alone and no substance. Considering each individual sign, groups of signs, and all signs together, significant differences were found between the percentages of people with signs when the concentration was $>0.25 \mathrm{mg}$ of ethanol per liter of breath air vs. $\leq 0.25 \mathrm{mg} / \mathrm{l}^{3}$. An association between this alcohol concentration and impairment was established previously; thus, we can conclude that these signs are useful for detecting impairment due to alcohol.

\subsection{Sign-positive drivers according to substance group}

More drivers with THC detected in their OF showed signs of impairment $(12.6 \%)$ than drivers without substances $(1.0 \% ; x 2=107.02 ; \mathrm{p}<0.0001)$, but the results were similar to those of drivers with alcohol detected in breath air $(9.5 \%$; $\mathrm{X} 2=1.15 ; \mathrm{p}>0.05)$. The group positive for $\mathrm{THC}+$ alcohol had a higher percentage of impaired drivers (28.1\%) than the group positive for THC alone $(12.6 \%$; $x 2=5.53 ; p<0.05$, Table 1$)$. Eye signs $(9.9 \%)$ were the most frequently observed

\footnotetext{
${ }^{3}$ Supplementary Table 1 . The proportion of impaired drivers was significantly related to breath alcohol concentration.
} 
signs in the THC group, followed by attitude signs (7.1\%) and body appearance signs $(4.3 \%)$. Figure 1 shows the percentages of drivers with observed signs of impairment (total signs and group signs) for each positive substance group. No differences were found between the THC group and the alcohol group for total signs or each individual group of signs. When alcohol and THC were present together, the percentage of drivers observed as being impaired increased significantly compared to the alcohol group or THC group. The result was the same for total signs, attitude signs, and eye signs.

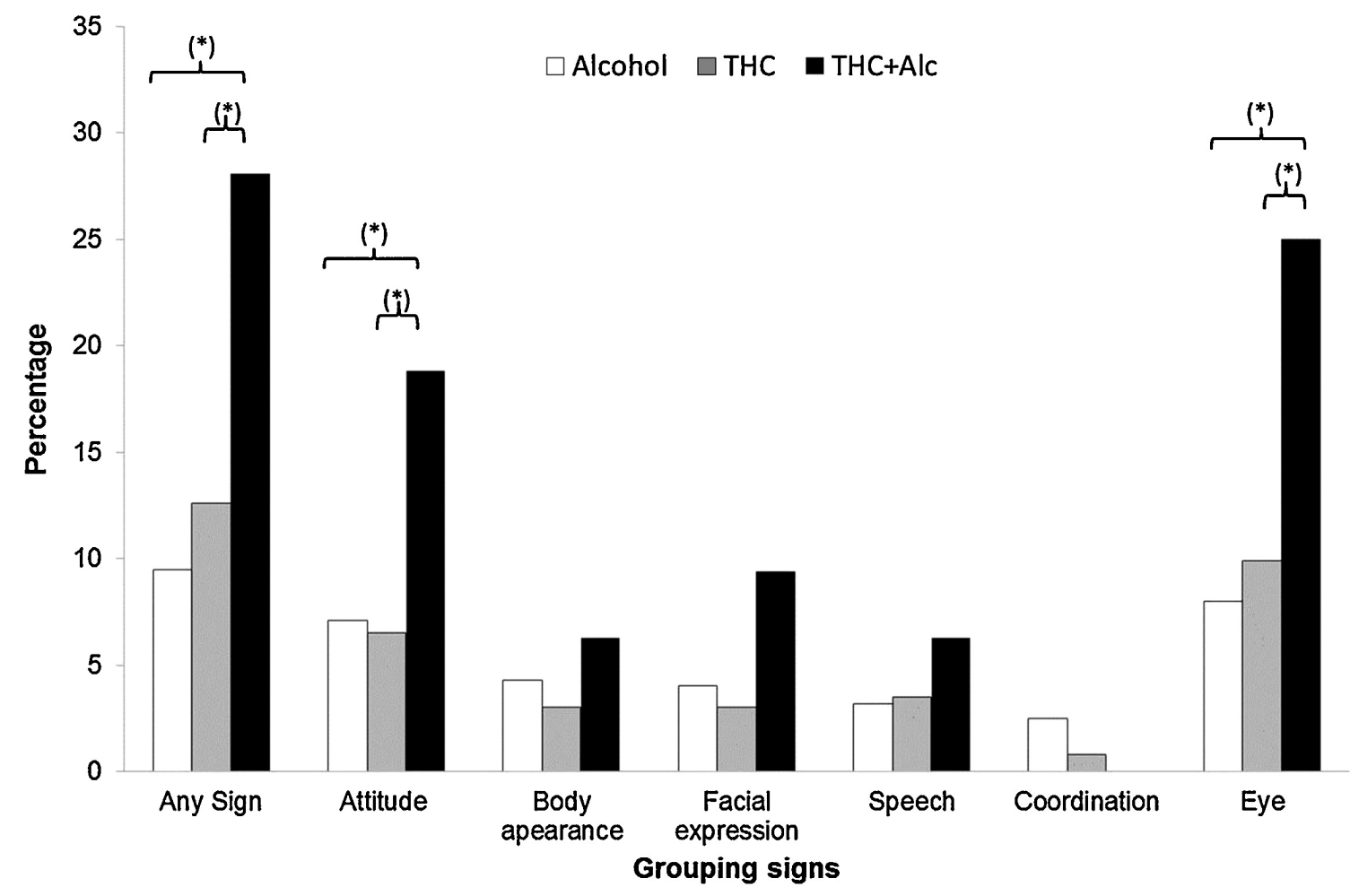

Figure 1 percentage of drivers with observed signs of impairment in each positive substance group. $* \mathrm{p}<0.05$ between groups. 
Table 1 Percentage of drivers with observed signs of impairment (any signs, group signs and individual signs)

\begin{tabular}{|c|c|c|c|c|}
\hline \multirow[t]{2}{*}{ Observed sign } & \multicolumn{4}{|c|}{ Detected substance } \\
\hline & $\begin{array}{l}\text { None } \\
(\mathrm{N}=2146)\end{array}$ & $\begin{array}{l}\text { THC } \\
(N=253)\end{array}$ & $\begin{array}{l}\text { Alcohol } \\
(\mathrm{N}=201)\end{array}$ & $\begin{array}{l}\text { THC+alcohol } \\
(\mathrm{N}=32)\end{array}$ \\
\hline Any sign $(\%)$ & 1.0 & 12.6 & 9.5 & 28.1 \\
\hline Attitude signs of impairment (\%) & 0.3 & 7.1 & 6.5 & 18.8 \\
\hline Nervous (\%) & 0.2 & 4.3 & 2.0 & 9.4 \\
\hline Euphoric (\%) & 0.0 & 0.4 & 1.0 & 0.0 \\
\hline Provocative (\%) & 0.0 & 0.8 & 0.0 & 3.1 \\
\hline Tearful (\%) & 0.0 & 0.0 & 1.0 & 0.0 \\
\hline Sleepy/drowsy (\%) & 0.0 & 2.0 & 3.0 & 9.4 \\
\hline Scratching his/her face (\%) & 0.0 & 0.0 & 0.0 & 3.1 \\
\hline Doesn't understand $(\%)$ & 0.0 & 0.0 & 0.5 & 3.1 \\
\hline Body appearance signs of impairment (\%) & 0.2 & 4.3 & 3.0 & 6.3 \\
\hline Trembling (\%) & 0.0 & 1.6 & 0.0 & 0.0 \\
\hline Perspiration (\%) & 0.0 & 0.4 & 0.0 & 0.0 \\
\hline Restlessness (\%) & 0.2 & 2.4 & 2.5 & 3.1 \\
\hline Superficial breathing $(\%)$ & 0.0 & 0.0 & 1.0 & 3.1 \\
\hline Facial expression signs of impairment (\%) & 0.2 & 4.0 & 3.0 & 9.4 \\
\hline Blinking (\%) & 0.2 & 2.4 & 2.5 & 6.3 \\
\hline Red nose $(\%)$ & 0.0 & 0.0 & 1.0 & 0.0 \\
\hline Sniffing (\%) & 0.0 & 0.0 & 0.0 & 0.0 \\
\hline Swallowing saliva (\%) & 0.0 & 0.4 & 0.0 & 3.1 \\
\hline Joint smell (\%) & 0.0 & 1.6 & 0.0 & 0.0 \\
\hline Speech signs of impairment (\%) & 0.2 & 3.2 & 3.5 & 6.3 \\
\hline Talkativeness (\%) & 0.0 & 1.2 & 2.0 & 3.1 \\
\hline Difficulties in speech (\%) & 0.0 & 0.4 & 1.0 & 0.0 \\
\hline Low tone $(\%)$ & 0.1 & 1.6 & 0.5 & 3.1 \\
\hline Coordination signs of impairment (\%) & 0.0 & 0.8 & 2.5 & 0.0 \\
\hline Staggering $(\%)$ & 0.0 & 0.0 & 1.0 & 0.0 \\
\hline No coordinated movements (\%) & 0.0 & 0.8 & 1.0 & 0.0 \\
\hline General trembling $(\%)$ & 0.0 & 0.0 & 0.5 & 0.0 \\
\hline Legs trembling $(\%)$ & 0.0 & 0.0 & 0.0 & 0.0 \\
\hline Eye signs of impairment (\%) & 0.7 & 9.9 & 8.0 & 25.0 \\
\hline Reddened conjunctive (\%) & 0.6 & 9.5 & 8.0 & 21.9 \\
\hline Brusque eye movement (\%) & 0.0 & 0.8 & 0.5 & 3.1 \\
\hline Wide nystagmus (\%) & 0.0 & 0.4 & 0.5 & 6.3 \\
\hline Nystagmus $45^{\circ}(\%)$ & 0.0 & 0.8 & 1.0 & 3.1 \\
\hline Nystagmus $30^{\circ}(\%)$ & 0.0 & 0.8 & 0.5 & 3.1 \\
\hline Pupil diameter (\%) & 0.0 & 0.8 & 0.0 & 0.0 \\
\hline Dilated pupil (\%) & 0.0 & 0.4 & 0.5 & 6.3 \\
\hline Slow pupil reaction (\%) & 0.0 & 1.6 & 0.5 & 3.1 \\
\hline
\end{tabular}




\subsection{Sign-positive drivers and cut-offs in the DRUID project}

Using the cut-off proposed in the DRUID project (27 $\mathrm{ng}$ of THC per $\mathrm{ml}$ of OF) in the group where THC was detected alone $(n=253)$, the sensitivity [95\% CI] based on observed signs of impairment was 19.3 [12.2-26.3], specificity [95\% CI] 94.9 [90.5-99.3], positive predictive value (PPV) 81.25\%, and negative predictive value (NPV) $50.68 \%$. Only $19.3 \%$ of the drivers with THC levels above the cut-off exhibited any sign, but significantly fewer drivers under the THC cutoff exhibited signs $(5.1 \% ; x 2=11.450 ; p=0.001)$.

Using the cut-off of $0.25 \mathrm{mg}$ of alcohol per liter of breath air, in the group positive for alcohol alone $(n=201)$ sensitivity based on observed signs of impairment was 27.7 [13.8-41.5], specificity 96.1 [92.7-99.5], PPV 68.42\%, and NPV $81.32 \%$. Among the drivers with alcohol levels above the cut-off, $27.7 \%$ exhibited any sign, but only $3.9 \%$ of drivers under the alcohol cut-off exhibited any sign $(x 2=23.76 ; p<0.0001)$.

No significant differences were found between the sensitivity of the observational method for THC $>27 \mathrm{ng} / \mathrm{ml}$ in the THC only group and the sensitivity for alcohol $>0.25 \mathrm{mg} / \mathrm{l}$ in the alcohol only group $\left(x^{2}=1.46 ; p>0.05\right)$. This finding is in accordance with the results obtained when applying cut-offs in the alcohol+THC group. When alcohol and THC are present together, the specificity of the test decreases and signs cannot be distinguished as being due to alcohol or THC. More drivers exhibit signs than expected among drivers under the cut-off for alcohol, and the same occurs with drivers under the cut-off for $\mathrm{THC}^{4}$.

\subsection{The Dräger Drug Test $® 5000$ on-site OF device vs. sign tests as a screening method for THC according to the DRUID cut-off}

The reliability of the observational test applied to THC was compared to the

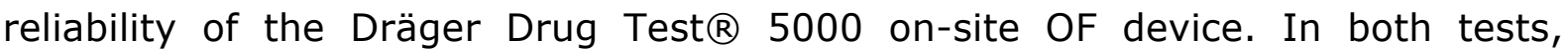
sensitivity and specificity were determined based on the results of collected OF

\footnotetext{
${ }^{4}$ Supplementary Table 2. Diagnostic value of the observation of signs of impairment and the onsite test, within each group of drivers, considering the established cut-off points.
} 
samples analyzed by UPLC-MS/MS and DRUID cut-off for THC $(27.0 \mathrm{ng} / \mathrm{ml})$. The sensitivity was much higher with the on-site OF device (76.3 [68.8-83.8] in the THC group and 76.5 [53.4-99.6] in the THC+alcohol group) than with the signs test (19.3 [12.2-26.3] in the THC group and 23.5 [0.4-46.6] in the THC+alcohol group). The specificity was lower, with wider confidence intervals, in the THC+alcohol group (80.0 [56.4-100.0] using the on-site OF device and 66.7 [39.5-93.9] using the signs test) than in the THC group, where the specificity was similar in both tests (93.2 [88.3-98.2] using the on-site OF device and 94.9 [90.5-99.3] using the signs test). In the two groups, accuracy was higher with the on-site OF device (84.2 [79.5-88.9] in the THC group and 78.1 [62.2-94.0] in the THC+alcohol group) than with the signs test (54.6 [48.2-60.9] and 43.75 [25.0-62.5], respectively).

\subsection{Cannabis and alcohol-positive drivers and prevalence of signs}

In order to investigate a possible relationship between the OF THC concentration and total observed signs, we determined four concentration intervals from the ROC analysis (AUC [IC 95\%]: 0.72 [0.62-0.82]): $\leq 3.00 \mathrm{ng} / \mathrm{ml} ; 3.01-25.00$ $\mathrm{ng} / \mathrm{ml} ; 25.01-100.00 \mathrm{ng} / \mathrm{ml}$; and $>100.00 \mathrm{ng} / \mathrm{ml}$.

The relationship between the OF THC concentration and signs of impairment was investigated based on any signs, ocular signs, and facial expressions (Table 2). With increasing OF THC concentrations, the drivers more frequently had one or more signs of impairment; between 0.01 and $3.00 \mathrm{ng}$ of THC per $\mathrm{ml}$ of OF, the proportion of impaired drivers was $2.9 \%$, gradually increasing to $22.5 \%$ at THC concentrations $>100 \mathrm{ng} / \mathrm{ml}\left(x^{2}=15.15 ; p<0.01\right)$. The prevalence of impairment with regard to THC concentration also differed for the presence of ocular signs $\left(x^{2}=18.05 ; p<0.0001\right)$ and facial expression signs $\left(x^{2}=10.68 ; p<0.05\right)$.

The percentage of drivers with one or more signs and OF THC concentration $>100.00 \mathrm{ng} / \mathrm{ml}(22.5 \%)$ roughly corresponded to the percentage of impaired drivers among drivers with breath alcohol concentration $>0.25 \mathrm{mg} / \mathrm{l}(27.7 \%)$. When alcohol and THC were present together, the frequency of drivers with signs of impairment increased compared to drivers positive for alcohol only. Similarly, 
alcohol increased the risk of exhibiting signs in each THC concentration range. No drivers were found with THC concentrations $>100 \mathrm{ng} / \mathrm{ml}$ in OF and alcohol $>0.25$ $\mathrm{mg} / \mathrm{l}$ simultaneously (Table 3 ). See some considerations about statistical power in the supplementary material ${ }^{5}$.

The risk of exhibiting signs of impairment was estimated by logistic regression for any sign and for each grouping of signs of impairment ${ }^{6}$. The adjusted OR (adjusted for gender, driver's age, road type, and period of time) for all signs (drivers free of substances were used as a reference) was: 1.70 (95\% CI: $0.22-$ 13.12) for drivers with THC concentrations $\leq 3.00 \mathrm{ng} / \mathrm{ml}$ in OF; 3.78 (1.50-9.55) for THC concentration $3.01-25.00 \mathrm{ng} / \mathrm{ml} ; 7.22(3.12-16.67)$ for THC concentration 25.01-100 ng/ml; and 16.55 (8.69-31.54) for THC concentration $>100 \mathrm{ng} / \mathrm{ml}$. For drivers with alcohol in concentrations of $0.01-0.25 \mathrm{mg} / \mathrm{l}$ in breath air, the OR was 3.26 (1.56-6.82); the OR was 28.97 (13.90-60.37) for alcohol concentrations $>0.25 \mathrm{mg} / \mathrm{l}$ of breath air.

The lack of interaction effects between THC and alcohol in the logistic regression analysis shows an independent contribution of alcohol and cannabis in the presence of signs of impairment. When alcohol and THC are present together, the effects could be described as comparable to the sum of the effects when alcohol and THC are consumed separately.

\footnotetext{
${ }^{5}$ Supplementary material 3: Statistical power and sample size.

${ }^{6}$ Supplementary Table 3. Risk of having signs of impairment associated with the consumption of cannabis and/or alcohol.
} 


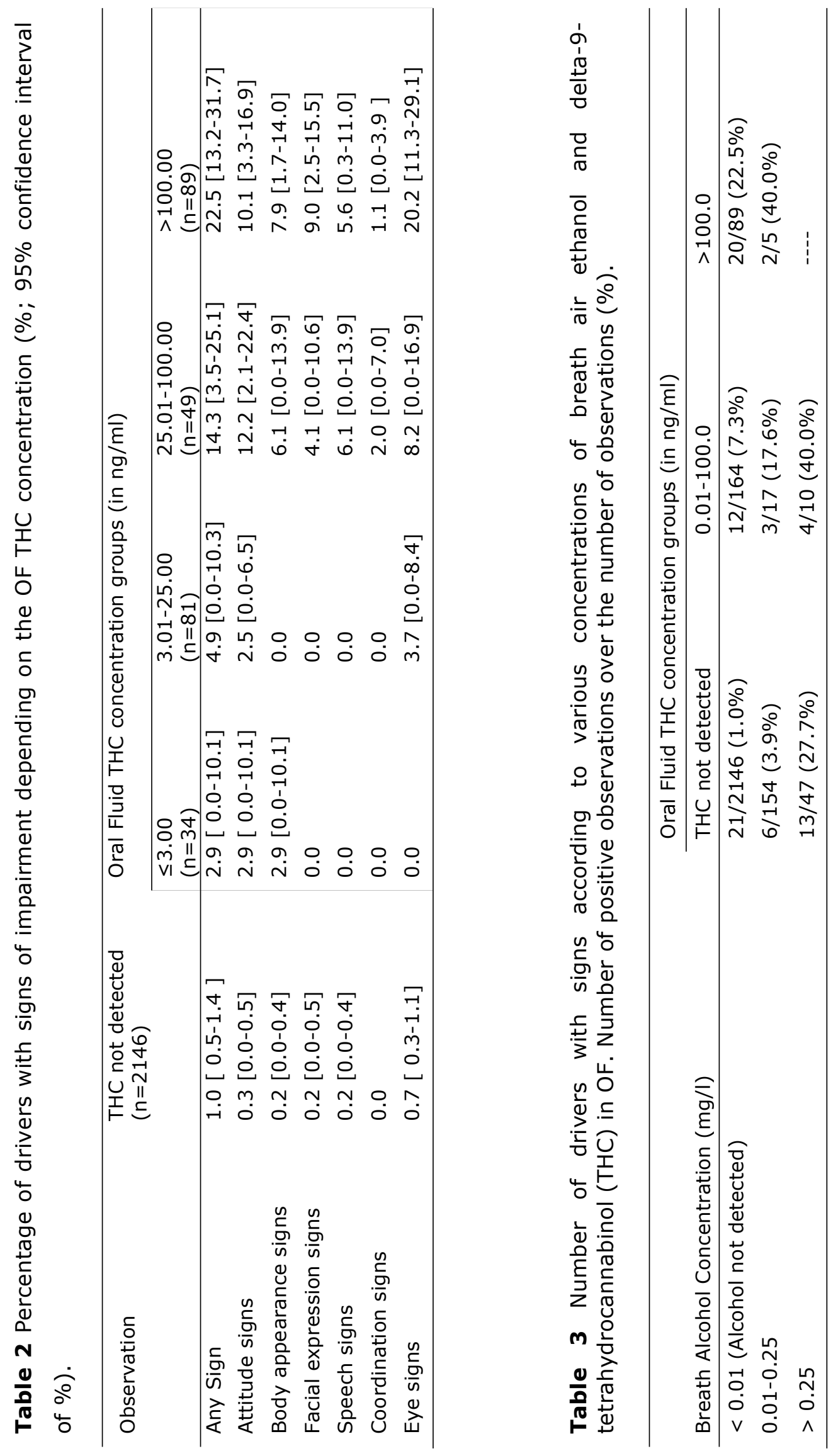




\section{Discussion}

This study shows the superiority of on-site OF devices versus the observation of signs of impairment for detecting drivers who have been exposed to THC. In addition, a positive concentration-effect relationship was found between OF THC concentration and impairment. The study was carried out on a representative random sample of Spanish drivers from a real-life roadside survey.

Currently, evidence-gathering technology for drugs (including THC) is sufficiently advanced and police officers can administer a simple on-site saliva test to obtain an indication of drug use nearly as easily as the preliminary breath test for alcohol, but more time is required to complete the test and it is more expensive than testing for alcohol. In addition, OF can be used in the confirmatory test, providing a non-invasive procedure and avoiding some ethical problems associated with blood or urine sampling. THC has traditionally been a problematic substance for immunoassay devices, but cut-off concentrations have decreased [33] and, despite the fact that results may be better for substances other than THC, the study shows a higher sensitivity for the on-site screening device $(76.3 \%)$ than the observational test (19.3\%). Therefore, the observation of signs of impairment may be unnecessary for showing DUI of THC. On the other hand, signs of impairment can be present in the general driver population (without consuming substances) due to tiredness, diseases, or other chronic or acute factors, and be absent in people with a high level of some substances in their body fluids. For example, in controlled studies of THC, some participants have shown no impairment in motor control, even at THC serum concentrations $>40$ $\mathrm{ng} / \mathrm{ml}[34]$.

The low sensitivity of tests for impairment due to cannabis based on the observation of signs was reported previously $[13,35]$. Most studies have been controlled experimental studies or were carried out with drivers apprehended on suspicion of DUI of drugs [15]. As expected in a random roadside survey, the sensitivity was lower than in the previously mentioned studies. Our study shows the limited reliability of observing signs of impairment in a roadside survey to detect drivers who have been exposed to cannabis. Only a fifth of these drivers were detected. Notably, the cut-off used for THC (27 ng/ml in OF) has been a 
plausible tool for statistical analyses. However, regardless of whether this cut-off point is reached, it should not be considered definite proof of impairment or lack of impairment.

Despite the low sensitivity, significance was reached when comparing results of the observational test using the cut-off for THC $(27 \mathrm{ng} / \mathrm{ml})$. This result opens up the possibility of using the observational test to avoid wasting resources in largescale screening, applying an OF sampling device only when signs of impairment are present. However, this study reveals underestimation of the percentage of drivers under the effects of cannabis in the random real-life roadside test. We are missing four out of every five drivers who have THC concentrations $>27$ $\mathrm{ng} / \mathrm{ml}$ when alcohol is not present. An important improvement in the sensitivity for SFSTs in DUI detection was reported when officers underwent an improved training program [36]. The police officers were trained for this study, and even though a small improvement may occur with subsequent training programs, a great increase in sensitivity is not expected, even with subsequent training programs. Notably, using OF devices, the observed prevalence of THC is still underestimated due to false-negative cases [37]. On the other hand, the observational test was not able to discriminate between performance impairments resulting from THC or alcohol. When breath tests are positive, the impairment effects can be attributed only to alcohol if THC or other substances are not being investigated. This is an important disadvantage to deterring DUI of cannabis, considering that drivers also need to be persuaded that they are at risk of their cannabis use being detected [38]. Considering the results, selection criteria for using on-site screening devices based on the observation of signs would notably reduce the sensitivity and reliability of the whole process, which will also be evaluated to determine whether it reduces accident risk and dissuades drivers from DUI of cannabis. Random traffic testing in Victoria, Australia, showed that on-site screening devices perform well as a deterrent, leading to a marked decrease in the prevalence of THC, MDMA, and methamphetamine, the three drugs covered in the state's legislation [37].

A relationship was found between the THC concentration in OF and the proportion of drivers showing signs of impairment. Also, as alcohol concentrations in breath air increased, the proportion of impaired drivers was 
significantly related to concentrations. This finding is in line with previous experimental and epidemiological studies showing that cannabis impairs driving performance in a concentration-related manner $[4,6,25]$. Considering the results obtained by Bramness et al. [15] in particular, the present results strengthen the hypothesis that a similar distribution of drug concentration exists in OF and whole blood in a population related by the prevalence of signs of impairment $[20,24]$.

A significantly higher risk of being observed as impaired was found in the groups with elevated OF concentrations of THC after adjusting for background variables. The OR was comparable to the OR found for different alcohol concentrations in breath air in the same population. Logistic regression showed an independent contribution of alcohol and cannabis to impairment. The results of this and previous studies are relevant when considering the concentration limits of substances for driving [15,39]; other studies have proposed a concentration of THC below which driving is not impaired [8]. On the other hand, the additive effects of both substances at high concentrations in the OF would justify none of the drivers being found with $>100 \mathrm{ng} / \mathrm{ml}$ of THC in OF and $>0.25 \mathrm{mg} / \mathrm{l}$ of alcohol in breath air simultaneously.

The low sensitivity of the observational sign test in detecting cannabis use has been previously reported $[13,14]$; as has the possibility of improving the sensitivity when the test is supplemented with two questions about recent drug use [27]. However, we had no reliable information about a subject's intake of cannabis; neither the dose nor the time was known, and the influence of possible effects due to tolerance is uncertain. Although drivers were asked about substance use, the information they provided was not useful. Of the 285 drivers for whom THC was detected, $40 \%$ reported not having used cannabis or related substances. Despite the participation in the study being voluntary and anonymity being guaranteed, the OF test for drugs was mandatory for all drivers and the drivers found to be positive were fined. In these circumstances drivers could have lied as a last resort to avoid a fine.

The main advantage of this study is that it was conducted in a real-life context, involving a random sample of drivers and using oral fluid as the matrix in the 
confirmation analysis. The random selection method avoids the selection bias that exists when drivers previously suspected of being under the effects of substances are included in the study [15].

As previously described in blood [15], this study shows that the prevalence of drivers with signs of impairment observable by police officers directly correlates with the concentration of THC in OF. Therefore, levels of impairment due to THC could be established as a direct function of OF concentrations without the need to consider blood concentrations of THC. Notably, in some countries, saliva/OF is currently the matrix used in on-road traffic checks for drugs and will probably be common in many countries in the future. The low sensitivity and specificity of signs of impairment raises the question of their rationale for use, as we now have various roadside devices that allow screening for the presence of drugs with reasonably good accuracy. Even more, the relevance of these signs should be questioned; in some countries, such as Germany and Spain, the presence of signs of impairment after a positive drug screening results in a more severe sanction.

\section{Role of Funding Source}

Funding for this study was provided by grants from the EU 6th Framework Programme, DRUID project, DGT (No. Exp.: 0100DGT21348), and the Instituto de Salud Carlos III, Redes Temáticas de Investigación Cooperativa, Red de Trastornos Adictivos RD06/0001/0020 and RD12/0028/0012. The funding sources had no further role in the study design; in the collection, analysis and interpretation of data; in the writing of the report; or in the decision to submit the paper for publication. The content is solely the responsibility of the authors and does not necessarily represent the official views of the funding agencies.

\section{Contributors}

Authors Fierro, Gonzalez-Luque, and Alvarez participated in the research design. Author González-Luque supervised the field trial. Author Fierro performed the 
data analysis. Authors Fierro and Alvarez wrote the initial draft of the manuscript, and all authors contributed to and have approved the final manuscript.

\section{Conflict of Interest}

The authors declare no conflict of interest. Dr F. J. Alvarez has received grants for speeches on pharmaceutical products.

\section{Acknowledgements}

This study was made possible thanks to the collaboration of the traffic police: The Civil Guard, a traffic police unit of Cataluña, and 16 units of local police. We also wish to express our thanks to the staff of the laboratory of the Forensic Toxicology Service of the University of Santiago de Compostela who carried out the toxicological analysis by LC-MS/MS quantification. 


\section{References}

1. Walsh, J.M., de Gier, J.J., Christopherson, A.S., Verstraete, A.G., 2004. Drugs and driving. Traffic Inj Prev. 5, 241-253.

2. Houwing, S., Hagenzieker, M., Mathijssen, R., Bernhoft, I.M., Hels, T., Janstrup, K., Van der Linden, T., Legrand, S.A., Verstraete, A., 2011. Prevalence of Alcohol and Other Psychoactive Substances in Drivers in General Traffic. Part I: General Results and Part II: Country Reports, DRUID Deliverable 2.2.3. Available on line at: http://www.druid-project.eu/Druid/EN/deliverales-

list/downloads/Deliverable_2_2_3_Part1.pdf?_blob=publicationFile and http://www.druid-project.eu/Druid/EN/deliveraleslist/downloads/Deliverable_2_2_3_Part2.pdf?_blob=publicationFile (accesses 09.06.14).

3. European Monitoring Centre for Drugs and Drug Addiction, 2012. Driving Under the Influence of Drugs, Alcohol and Medicines in Europe - findings from the DRUID project, Lisbon. Available on line at: http://www.emcdda.europa.eu/publications/thematic-papers/druid (accesses 09.06.14).

4. Drummer, O.H., Gerostamoulos, J., Batziris, H., Chu, M., Caplehorn, J., Robertson, M.D., Swann, P., 2004. The involvement of drugs in drivers of motor vehicles killed in Australian road traffic crashes. Accid Anal Prev. 36, 239-248.

5. Asbridge, M., Hayden, J.A., Cartwright, J.L., 2012. Acute cannabis consumption and motor vehicle collision risk: systematic review of observational studies and metaanalysis. BMJ. 344, e536.

6. Ramaekers, J.G., Berghaus, G., van Laar, M., Drummer, O.H., 2004. Dose related risk of motor vehicle crashes after cannabis use. Drug Alcohol Depend. 73, 109-119.

7. Laumon, B., Gadegbeku, B., Martin, J.L., Biecheler, M.B., 2005. Cannabis intoxication and fatal road crashes in France: population based case-control study. BMJ. 331, 1371.

8. Grotenhermen, F., Leson, G., Berghaus, G., Drummer, O.H., Kruger, H.P., Longo, M., Moskowitz, H., Perrine, B., Ramaekers, J.G., Smiley, A., Tunbridge, R., 2007. Developing limits for driving under cannabis. Addiction. 102, 1910-1917.

9. NHTSA, 2001. Development of a Standardized Field Sobriety Test (SFST). Training Management System. DOT HS 809 400. Available at: http://www.nhtsa.gov/people/injury/alcohol/sfst/introduction.htm (accesses 09.06.14). 
10. Burns, M., Moskowitz, H., 1977. Psychophysical Test for DWI Arrest. U.S. Department of Transportation, National HighwayTraffic safety Administration, Washington, D.C.

11. Burns, M., 1987. Sobriety test for the presence of drugs. Alcohol Drugs Driv. 3, 2529.

12. Raes, E., Van den Neste, T., Verstraete, A.G., 2008. Drug use, Impaired Driving and Traffic Accidents. European Monitoring Centre for Drugs and Drug Addiction (EMCDDA), Lisbon.

13. Papafotiou, K., Carter, J.D., Stough, C., 2005a. An evaluation of the sensitivity of the Standardised Field Sobriety Tests (SFSTs) to detect impairment due to marijuana intoxication. Psychopharmacology (Berl). 180, 107-114.

14. Papafotiou, K., Carter, J.D., Stough, C., 2005b. The relationship between performance on the standardised field sobriety tests, driving performance and the level of Delta9-tetrahydrocannabinol (THC) in blood. Forensic Sci Int. 155, 172-178.

15. Bramness, J.G., Khiabani, H.Z., Morland, J., 2010. Impairment due to cannabis and ethanol: clinical signs and additive effects. Addiction. 105, 1080-1087.

16. Boskers, W.M., Theunissen, E.L., Conen, S., Kuypers, K.P.C., Jeffery, W.K., Walls, H.C., Kauert, G.F., Toennes, S.W., Moeller, M.R., Ramaekers, J.G., 2012. A placebocontrolled study to asses Standardized Field Sobriety Tests performance during alcohol and cannabis intoxication in heavy cannabis users and accuracy of point of collection testing devices for detecting THC in oral fluid. Psychopharmacology. 223, 439-446.

17. Wille, S.M., Raes, E., Lillsunde, P., Gunnar, T., Laloup, M., Samyn, N., Christophersen, A.S., Moeller, M.R., Hammer, K.P., Verstraete, A.G., 2009. Relationship between oral fluid and blood concentrations of drugs of abuse in drivers suspected of driving under the influence of drugs. Ther Drug Monit. 31, 511-519.

18. Choo, R.E., Huestis, M.A., 2004. Oral fluid as a diagnostic tool. Clin Chem Lab Med. $42,1273-1287$.

19. Verstraete, A.G., 2005. Oral fluid testing for driving under the influence of drugs: history, recent progress and remaining challenges. Forensic Sci Int. 150, 143-150.

20. Gjerde, H., Verstraete, A.G., 2011. Estimating equivalent cutoff thresholds for drugs in blood and oral fluid using prevalence regression: a study of tetrahydrocannabinol and amphetamine. Forensic Sci Int. 212, e26-30. 
21. Crouch, D.J., 2005. Oral fluid collection: the neglected variable in oral fluid testing. Forensic Sci Int. 150, 165-173.

22. Gjerde, H., Mordal, J., Christophersen, A.S., Bramness, J.G., Morland, J., 2010. Comparison of drug concentrations in blood and oral fluid collected with the Intercept sampling device. J Anal Toxicol. 34, 204-209.

23. Vindenes, V., Lund, H.M., Andresen, W., Gjerde, H., Ikdahl, S.E., Christophersen, A.S., Oiestad, E.L., 2012. Detection of drugs of abuse in simultaneously collected oral fluid, urine and blood from Norwegian drug drivers. Forensic Sci Int.

24. Gjerde, H., Verstraete, A., 2010. Can the prevalence of high blood drug concentrations in a population be estimated by analysing oral fluid? A study of tetrahydrocannabinol and amphetamine. Forensic Sci Int. 195, 153-159.

25. Ramaekers, J.G., Moeller, M.R., van Ruitenbeek, P., Theunissen, E.L., Schneider, E., Kauert, G., 2006. Cognition and motor control as a function of Delta9-THC concentration in serum and oral fluid: limits of impairment. Drug Alcohol Depend. 85, 114-122.

26. Gomez-Talegon, T., Fierro, I., Gonzalez-Luque, J.C., Colas, M., Lopez-Rivadulla, M., Javier Alvarez, F., 2012. Prevalence of psychoactive substances, alcohol, illicit drugs, and medicines, in Spanish drivers: a roadside study. Forensic Sci Int. 223, 106-113.

27. Mathijssen, R., Houwing, S., 2005. The prevalence and relative risk of drink and drug driving in the Netherlands: a case-control study in the Tilburg pólice district. Leidschendam: SWOV. http://www.swov.nl/rapport/r-2005-09.pdf (accesses 16.06.14).

28. Möller, M., 1998. Drogenerkennung im Strassenverkehr. Berichte der Bundesanstalt für Strassenwesen, Mensch und Sicherheit M 96. Bundesanstalt für Strassenwesen BASt, Bergisch Gladbach.

29. Concheiro, M., de Castro, A., Quintela, O., Cruz, A., Lopez-Rivadulla, M., 2008. Determination of illicit and medicinal drugs and their metabolites in oral fluid and preserved oral fluid by liquid chromatography-tandem mass spectrometry. Anal Bioanal Chem. 391, 2329-2338.

30. DRUID, 2007. Working Paper. Uniform Design and Protocols for Carrying Out CaseControl Studies. Available on line at: http://www.druidproject.eu/Druid/EN/deliverales-list/downloads/deliverable_2_1_2.pdf?_blob=public ationFile (accesses 09.06.14). 
31. Koski, A., Vuori, E., Ojanpera, I., 2005. Relation of postmortem blood alcohol and drug concentrations in fatal poisonings involving amitriptyline, propoxyphene and promazine. Hum Exp Toxicol. 24, 389-396.

32. European Commission. Mobility and Transport: Road Safety, 2014. Legal alcohol limits for the general driver population, Available on line at http://ec.europa.eu/transport/road_safety/specialist/knowledge/alcohol/prevalence_ amp_rate_of_alcohol_consumption/the_legal_limit_en.htm (accesses 21.07.14).

33. Pil, K., Verstraete, A., 2008. Current developments in drug testing in oral fluid. Ther Drug Monit. 30, 196-202.

34. Hunault, C.C., Mensinga, T.T., Bocker, K.B., Schipper, C.M., Kruidenier, M., Leenders, M.E., de Vries, I., Meulenbelt, J., 2009. Cognitive and psychomotor effects in males after smoking a combination of tobacco and cannabis containing up to $69 \mathrm{mg}$ delta9-tetrahydrocannabinol (THC). Psychopharmacology (Berl). 204, 85-94.

35. Tzambazis, K., Stough, C., 2002. The SFST and driving ability. Are they related? In: D.R. Mayhew, C. Dussault (Eds.), ICADTS, Montreal. $16^{\text {th }}$ International Conference on Alcohol Drugs and Traffic safety. Proceedings 2, 397-400.

36. Walsh, J., Cangianelli, L., 2002. New training in detection of illicit drugs in drivers. In: Mayhew, D. R., Dussault, C. (Eds), Alcohol, drugs and traffic safety - T'2002, Société de I'assurance automobile du Québec, Québec, pp. 387-390.

37. Chu, M., Gerostamoulos, D., Beyer, J., Rodda, L., Boorman, M., Drummer, O.H., 2012. The incidence of drugs of impairment in oral fluid from random roadside testing. Forensic Sci Int. 215, 28-31.

38. Watling, C.N., Palk, G.R., Freeman, J.E., Davey, J.D., 2010. Applying Stafford and Warr's reconceptualization of deterrence theory to drug driving: can it predict those likely to offend? Accid Anal Prev. 42, 452-458.

39. Downey, L.A., King, R., Papafotiou, K., Swann, P., Ogden, E., Boorman, M., Stough, C., 2012. The effects of cannabis and alcohol on simulated driving: Influences of dose and experience. Accid Anal Prev. 50, 879-886. 


\section{Appendix A. Supplementary data}

${ }^{1}$ Supplementary Table 1 . The proportion of impaired drivers was significantly related to breath alcohol concentration.

\begin{tabular}{llll|l}
\hline \multirow{2}{*}{ Observation, y/n (\%) } & \multicolumn{3}{l}{ Breath Alcohol Concentration } & \multicolumn{2}{l}{ (Alcohol not detected) } & $0.01-0.25 \mathrm{mg} / \mathrm{l}$ & $>0.25 \mathrm{mg} / \mathrm{l}$ & $\mathrm{X}^{2} ; \mathrm{p}$-value \\
\cline { 2 - 5 } & $\begin{array}{l}<0.01 \\
\text { Any Signs }\end{array}$ & $6 / 154(3.9)$ & $13 / 47(27.7)$ & $200.64 ;<0.0001$ \\
Attitude \& behavior & $6 / 2146(0.3)$ & $4 / 154(2.6)$ & $9 / 47(19.1)$ & $210.98 ;<0.0001$ \\
Body appearance & $4 / 2146(0.2)$ & $1 / 154(0.6)$ & $5 / 47(10.6)$ & $118.89 ;<0.0001$ \\
Facial expression & $5 / 2146(0.2)$ & $3 / 154(1.9)$ & $3 / 47(6.4)$ & $45.14 ;<0.0001$ \\
Speech signs & $4 / 2146(0.2)$ & $3 / 154(1.9)$ & $4 / 47(8.5)$ & $76.23 ;<0.0001$ \\
Coordination signs & $0 / 2146(0.0)$ & $1 / 154(0.6)$ & $4 / 47(8.5)$ & $158.53 ;<0.0001$ \\
Eye signs & $15 / 2146(0.7)$ & $5 / 154(3.2)$ & $11 / 47(23.4)$ & $187.05 ;<0.0001$ \\
\hline
\end{tabular}




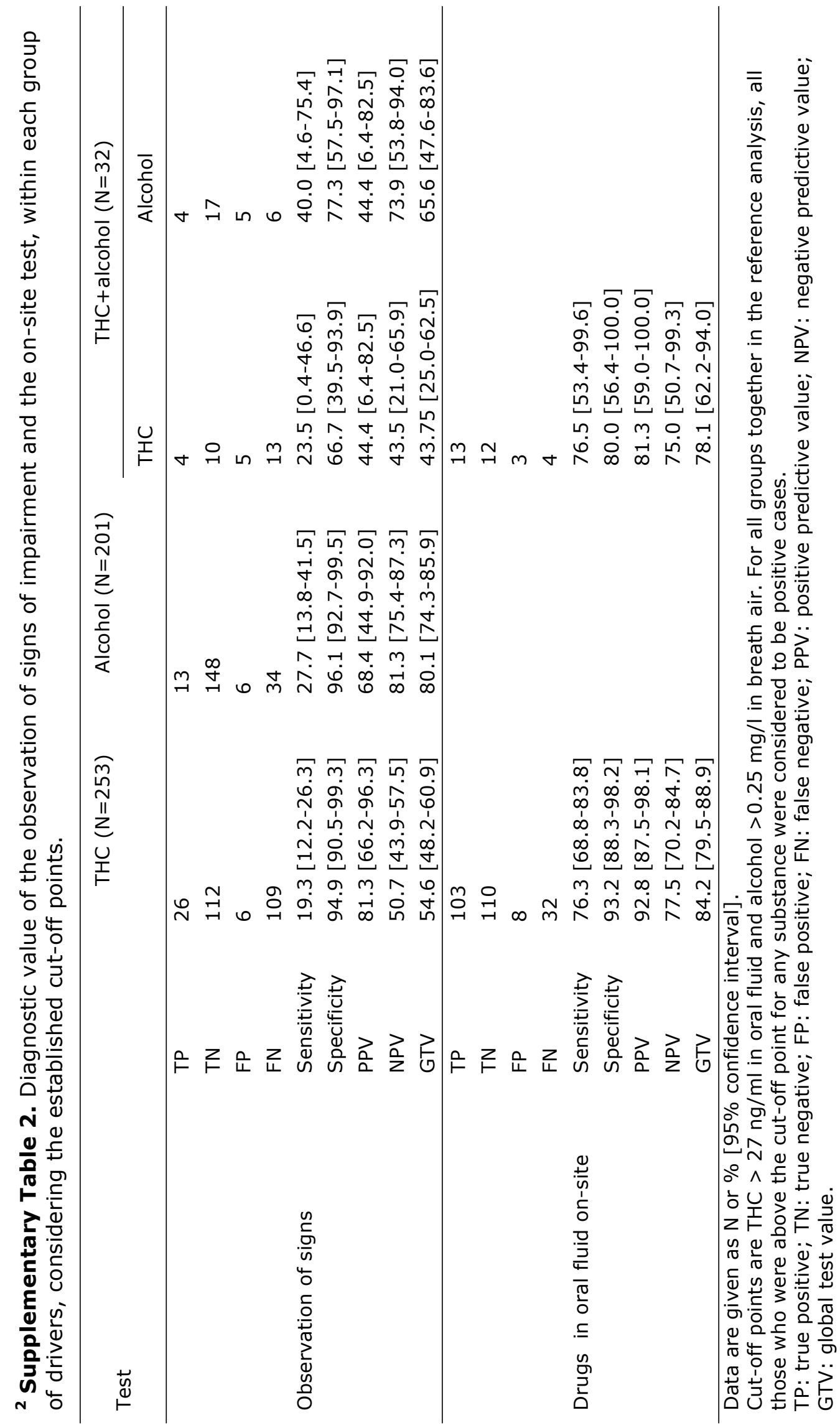




\section{${ }^{3}$ Supplementary materials: Statistical power and sample size.}

Based on the difference in outcomes between the reference groups (no substance and $1 \%$ of drivers with any sign of impairment) and the other groups (in THC group $16.6 \%$ of drivers with any sign of impairment; $9.5 \%$ in alcohol group and $28.1 \%$ in THC+alcohol group), the statistical power is $>80 \%$ ( $95 \%$ CI and two-sided test) for the detection of differences in any sign of impairment and eye signs of impairment when comparing the groups (THC, alcohol, and THC+alcohol) (See Table 1). This statistical power is also achieved when comparing the percentage of drivers with any signs according to various concentrations of THC (See Table 3) when alcohol is not detected. For the THC + alcohol group, the sample size limited the statistical power. 


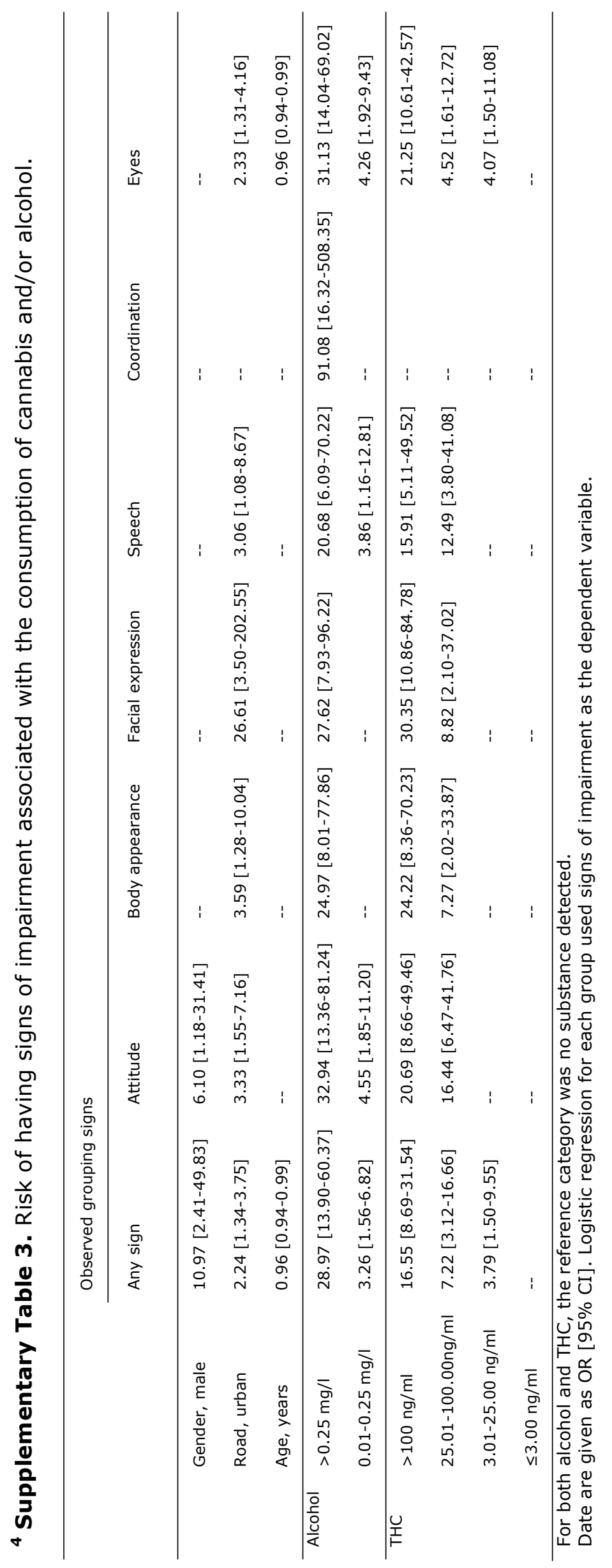


Capítulo 5

Agresividad vial en la población general [Road-rage in the general population]

Fierro, I., Gómez-Talegón, MT., Álvarez, FJ.

Gac Sanit, 2010; 24: 423-427 



\section{Resumen}

Objetivos: Analizar la prevalencia y los factores sociodemográficos asociados con la agresividad vial en la población.

Métodos: Se han realizado 2500 entrevistas a la población de Castilla y León de entre 14 y 70 años de edad. Se evaluó la agresividad vial en el año previo a la realización de la encuesta utilizando un test de ocho preguntas.

Resultados: El 31.1\% refirió haber vivido alguna situación de agresividad vial en el último año, y el $26.8 \%$ en más de una ocasión. El $2.6 \%$ fueron agresores viales «graves». Entre los conductores, la probabilidad de experimentar agresividad vial aumenta a medida que aumentan los miles de kilómetros conducidos a la semana (odds ratio $[\mathrm{OR}]=1.52$ ), es menor cuanto mayor es la edad del entrevistado $(O R=0.975)$ y es mayor en los hombres $(O R=1.287)$, en los que tienen estudios universitarios $(O R=1.408)$ y en los que viven en localidades de más de 10.000 habitantes $(O R=1.25)$.

Conclusiones: Los datos del presente estudio muestran que la agresividad vial afecta a casi un tercio de la población general de Castilla y León, lo que justificaría la adopción de medidas para su prevención y reducción.

\section{Palabras clave}

Accidentes de tráfico, Agresividad vial, Conducción de vehículos, España, Seguridad vial 


\section{Abstract}

Objective: To analyze the prevalence of road rage in the general population and the sociodemographic factors associated with this phenomenon.

Methods: A total of 2500 interviews were carried out in the population of Castile and Leon aged 14-70 years. Road rage was evaluated in the year prior to the survey using a test with eight questions.

Results: One-third (31.1\%) of the interviewees reported they had experienced a situation involving road rage during the previous 12 months (26.8\% on more than one occasion). Among these episodes, 2.6\% involved "serious" aggressors. In drivers, the probability of experiencing road rage increased in line with the number of kilometers driven per week (odds ratio $[O R]=1.52$ ), decreased as the age of the driver increased $(O R=0.975)$, and was highest in men $(O R=1.287)$, university graduates $(O R=1.408)$, and persons living in towns with over 10000 inhabitants $(\mathrm{OR}=1.25)$.

Conclusions: The results of this study show that road rage affects almost a third of the general population of Castile and Leon, which would amply justify the adoption of prevention and/or reduction measures.

\section{Keywords}

Traffic accidents, Road rage, Automobile driving, Spain, Road safety 


\section{Introducción}

Los accidentes de tráfico constituyen un enorme problema de salud pública. Aunque en los últimos años esta accidentalidad se ha reducido de manera notable en España, las cifras son aún demasiado altas. La tasa de víctimas de accidentes de tráfico por 100.000 habitantes se ha reducido de 384 en 2000 a 294 en 2008[1]. En 2005 se produjeron en España 316 víctimas de accidentes de tráfico por cada 100.000 habitantes, menos que en el conjunto de la Unión Europea (361). Ese año, las mayores cifras de víctimas de accidentes de tráfico se observaron en Eslovenia (728) y Austria (656), y las menores en Rumanía (39) y Bulgaria (143)[1].

En el ámbito internacional, la agresividad vial (en inglés road rage) ha resurgido como un tema emergente, que además es objeto de creciente interés en los medios de comunicación $[2,3]$. Su relevancia radica en que se ha observado que tanto víctimas (odds ratio $[\mathrm{OR}]=1.89$; intervalo de confianza 95\% $[\mathrm{IC} 95 \%]=1.39-2.39)$ como agresores $(\mathrm{OR}=1.82 ; \mathrm{IC} 95 \%=1.32-2.31)$ tienen un riesgo significativamente mayor de involucrarse en accidentes de tráfico [4]. La relación entre la agresividad vial y la adopción de determinadas conductas peligrosas en la conducción (por ejemplo, conducir a gran velocidad) explicaría el aumento en el riesgo de sufrir un accidente en el caso de los agresores; en el de las víctimas, la accidentalidad sería consecuencia de otro tipo de comportamiento que podría despertar la agresividad de otros conductores (como la no señalización de una maniobra, o la tendencia a circular muy lentamente en determinadas condiciones de tráfico) [4]. Por otra parte, la agresividad vial suele manifestarse como un proceso interactivo entre víctima y agresor [4], con intercambio de expresiones de hostilidad, lo que en ocasiones puede dificultar la distinción entre víctima y agresor, además de poder involucrar tanto al conductor del vehículo como a los pasajeros.

No hay una definición unívoca para la expresión «agresividad vial», pero su equivalente en inglés se ha utilizado con frecuencia para describir «un incidente en el cual un conductor o pasajero intenta intimidar, herir e incluso matar a otro conductor, pasajero o peatón, o dañar el vehículo de otra persona» $[2,3,5,6]$. 
Cerca de la mitad de los conductores $[6,7]$ refirieron haber experimentado agresividad vial en el último año, y en especial haber sido víctimas de la agresividad vial. Se ha observado con más frecuencia en los hombres, en los más jóvenes y en los que viven en grandes centros urbanos. La agresividad vial es más frecuente en las situaciones de congestión del tráfico y de conducción estresante $[3,6]$.

En España, la información disponible sobre agresividad vial es muy limitada. El presente estudio tiene como objeto analizar la prevalencia y los factores sociodemográficos asociados con la agresividad vial en la población general de Castilla y León.

\section{Métodos}

La población objeto del estudio fueron los habitantes de Castilla y León de edad comprendida entre los 14 y los 70 años [8], según las cifras oficiales de población de 2007 (padrón municipal de 2008, http://www.ine.es). El tamaño de la muestra se estableció en 2.500 entrevistas válidas. La distribución de las encuestas se realizó según estudios previos llevados a cabo con la misma población de referencia [9], teniendo en cuenta los siguientes estratos: tamaño del municipio, provincia, rangos de edad y sexo.

Los datos se obtuvieron mediante entrevistas personales realizadas a una muestra aleatoria, representativa de la población de Castilla y León, Ilevadas a cabo entre el 21 de abril y el 22 de mayo de 2008 por la empresa Telecyl. Posteriormente se efectuaron llamadas telefónicas de comprobación al 16,2\% de los entrevistados mayores de 16 años, y se rechazaron 16 encuestas incompletas o incoherentes. Los menores de 16 años no se incluyeron en el proceso de comprobación, ya que no se solicitó su número de teléfono (RD 1720/2007 de 21 de diciembre), pero ello no afectó a la comprobación de la veracidad de los datos según la metodología aplicada. El estudio fue aprobado por el Comité Ético de Investigación Clínica de la Facultad de Medicina de la Universidad de Valladolid. En el cuestionario, con el fin de evaluar la agresividad vial en el último año se incluyó un test de ocho preguntas $[5,6,10,11]$, ya utilizado en estudios previos. 
Las cuatro primeras preguntas van dirigidas a analizar la experiencia de la violencia en la conducción como víctima, y las cuatro restantes como agresor. Para ambas experiencias (como víctima y como agresor), el orden de los ítems refleja una progresión en la gravedad de la expresión o manifestación violenta: 1) insultos $\mathrm{y} / \mathrm{o}$ gestos obscenos; 2) intimidación $\mathrm{y} / \mathrm{o}$ amenazas; 3) daños intencionados en el vehículo; y 4) lesiones intencionadas al conductor del vehículo o a alguno de sus ocupantes (tabla 1). En cada uno de los ítems se preguntó, además, el número de veces que se había tenido esa experiencia durante el último año. El test fue traducido del inglés al español, y posteriormente se realizó una retrotraducción al inglés. La concordancia del texto en ambos idiomas fue acordada por dos traductores bilingües. Diferenciaremos dos categorías de agresores atendiendo a la gravedad de la agresión vial [4]: agresor leve, si respondió afirmativamente sólo al primer ítem de agresión, y agresor grave si señaló al menos uno de los tres últimos ítems de agresor.

El análisis estadístico se realizó con el programa SPSS 15.0, y para analizar la asociación entre variables categóricas se utilizó el test de $x^{2}$. La comparación entre medias se realizó con el test de la $\mathrm{t}$ de Student. Mediante análisis de regresión logística multinomial por pasos hacia delante, y de forma separada para conductores y no conductores, se determinó la influencia de cinco factores sociodemográficos, codificados como variables dicotómicas, en la agresividad vial: sexo (hombre/mujer), nivel de estudios universitarios (sí/no), estado civil (en pareja/sin pareja), situación laboral (trabaja sí/no), tamaño del municipio (menor o $\geq 10.000$ habitantes); además, se consideró la edad y los miles de kilómetros conducidos a la semana como covariables (variables continuas, la última de ellas utilizada sólo para los conductores). También mediante regresión logística se analizaron los factores asociados a ser víctima y a ser agresor, de forma separada: variables dependientes víctima (sí/no) en el primer caso y agresor (sí/no) en el segundo. Se consideró estadísticamente significativo un valor de $\mathrm{p} \leq 0.05$. 


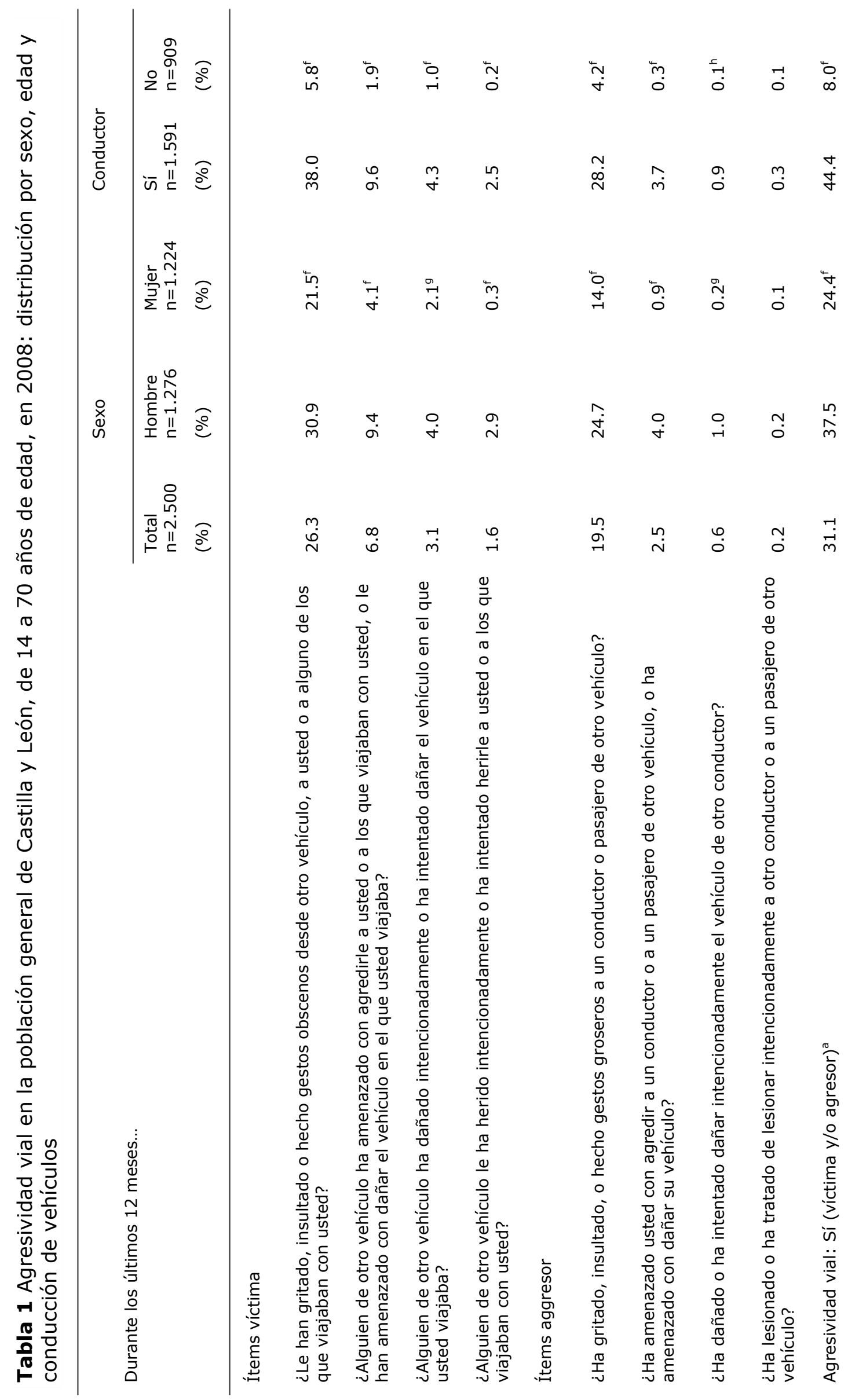




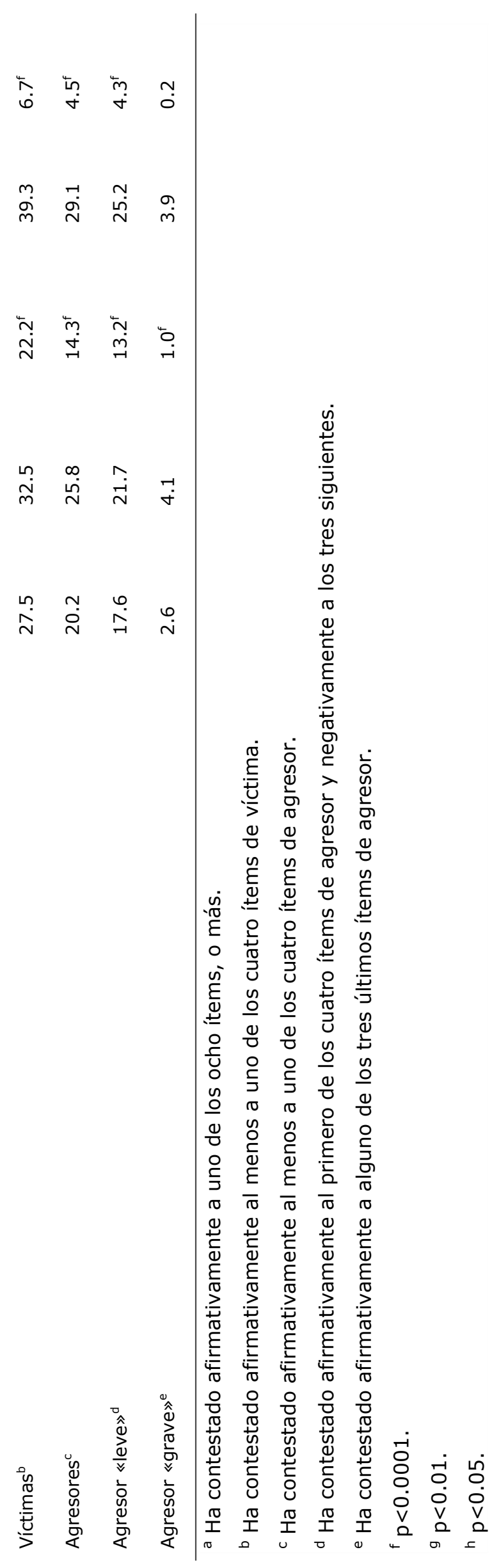




\section{Resultados}

La distribución de la muestra fue la siguiente: 1276 hombres y 1224 mujeres; 189 tenían edades comprendidas entre 14 y 19 años, 447 entre 20 y 29 años, 522 entre 30 y 39 años, 529 entre 40 y 49 años, 427 entre 50 y 59 años, y 386 entre 60 y 70 años; y el 63,6\% conduce vehículos.

El $31.1 \%$ de los entrevistados refirió haber vivido alguna situación de agresividad vial en el año previo a la realización de la entrevista. De éstos, el $16.5 \%$ tuvo experiencias tanto de víctima como de agresor; el $11.0 \%$ fue víctima de agresividad vial y el 3.6\% fue únicamente agresor. El 20.2\% de los entrevistados incurrió en algún comportamiento de agresividad vial: el 17.6\% fueron agresores viales «leves» (insultos y/o gestos obscenos) y el $2.6 \%$ agresores viales «graves».

Los distintos supuestos de agresividad vial en los últimos 12 meses (tabla 1) son más frecuentes entre los hombres que en las mujeres, difieren en función de la edad (la mayor frecuencia se observa en los de 20 a 29 años) y, son más frecuentes entre los conductores que entre los no conductores.

En la tabla 2 se presenta el número de veces que en el último año han referido las distintas situaciones de agresividad vial: una media de 8.33 en el último año, con más frecuencia $(p<0.05)$ los hombres que las mujeres y los que conducen $(8.75 \pm 9.48)$ que los que no conducen $(4.24 \pm 4.61 ; \mathrm{p}<0.001)$. La mayoría de las personas han experimentado varias situaciones de agresividad vial en el último año: el $26.8 \%$ en más de una ocasión, y de ellos el $8.7 \%$ diez veces o más.

El análisis de regresión logística (tabla 3) muestra que, para los no conductores, el único factor sociodemográfico influyente es la edad: la probabilidad de experimentar agresividad vial es menor cuanto mayor es la edad del entrevistado $(\mathrm{OR}=0.969 ; \mathrm{IC} 95 \%=0.956-0.983 ; \mathrm{p}<0.0001)$. En el caso de los conductores, la edad influye en el mismo sentido ( $O R=0.975$; IC95\%=0.967-0.983; $p<0.0001$ ), y también es más probable la experiencia de agresividad vial entre los hombres que entre las mujeres $(O R=1.287 ; \mathrm{IC} 95 \%=1.03-1.60 ; \mathrm{p}<0.05)$, entre los que tienen estudios universitarios que entre el resto de conductores $(O R=1.408$; 
IC95\%=1.121-1.773; $\mathrm{p}<0.01)$, y para los que viven en localidades de más de 10.000 habitantes $(\mathrm{OR}=1,250 ; \mathrm{IC} 95 \%=1.017-1.535 ; \mathrm{p}<0.05)$ que para los habitantes de localidades más pequeñas. La exposición a la conducción (miles de kilómetros conducidos a la semana) también aumenta la probabilidad de experimentar agresividad vial $(\mathrm{OR}=1.520 ; \mathrm{IC} 95 \%=1.202-1.921 ; \mathrm{p}<0.0001)$.

Analizando por una parte las respuestas como víctima (sí/no) y por otra las de agresor (sí/no), podemos observar (tabla 3) que en los no conductores la probabilidad de ser víctima de agresividad vial se incrementa en las localidades de más de 10.000 habitantes (OR=1.974; IC95\%=1.086-3.587; $p<0.05)$ y disminuye con la edad (OR=0.971; IC95\%=0.957-0.986; $\mathrm{p}<0.0001)$. La probabilidad de ser agresor también disminuye a medida que aumenta la edad (OR=0.955; IC95\%=0.935-0.975; p<0.0001). Para los conductores, la probabilidad de ser víctima es mayor entre los que poseen estudios universitarios $(\mathrm{OR}=1.366$; IC95\%=1.088-1.715; $\mathrm{p}<0.01)$ y para los que viven en localidades de más de 10.000 habitantes (OR=1.340; IC95\%=1.087-1.652; $\mathrm{p}<0.01)$, aumenta con los kilómetros conducidos a la semana $(O R=1.705$; IC95\%=1.350$2.154 ; \mathrm{p}<0.0001)$ y disminuye a medida que aumenta la edad $(O R=0.980$; IC95\%=0.972-0.988; $\mathrm{p}<0.0001)$. La probabilidad de que un conductor se comporte como agresor es mayor para los hombres (OR=1.619; IC95\%=1.281$2.045 ; \mathrm{p}<0.0001)$ y disminuye con la edad (OR=0.969; IC95\%=0.960-0.977; $\mathrm{p}<0.0001)$. 


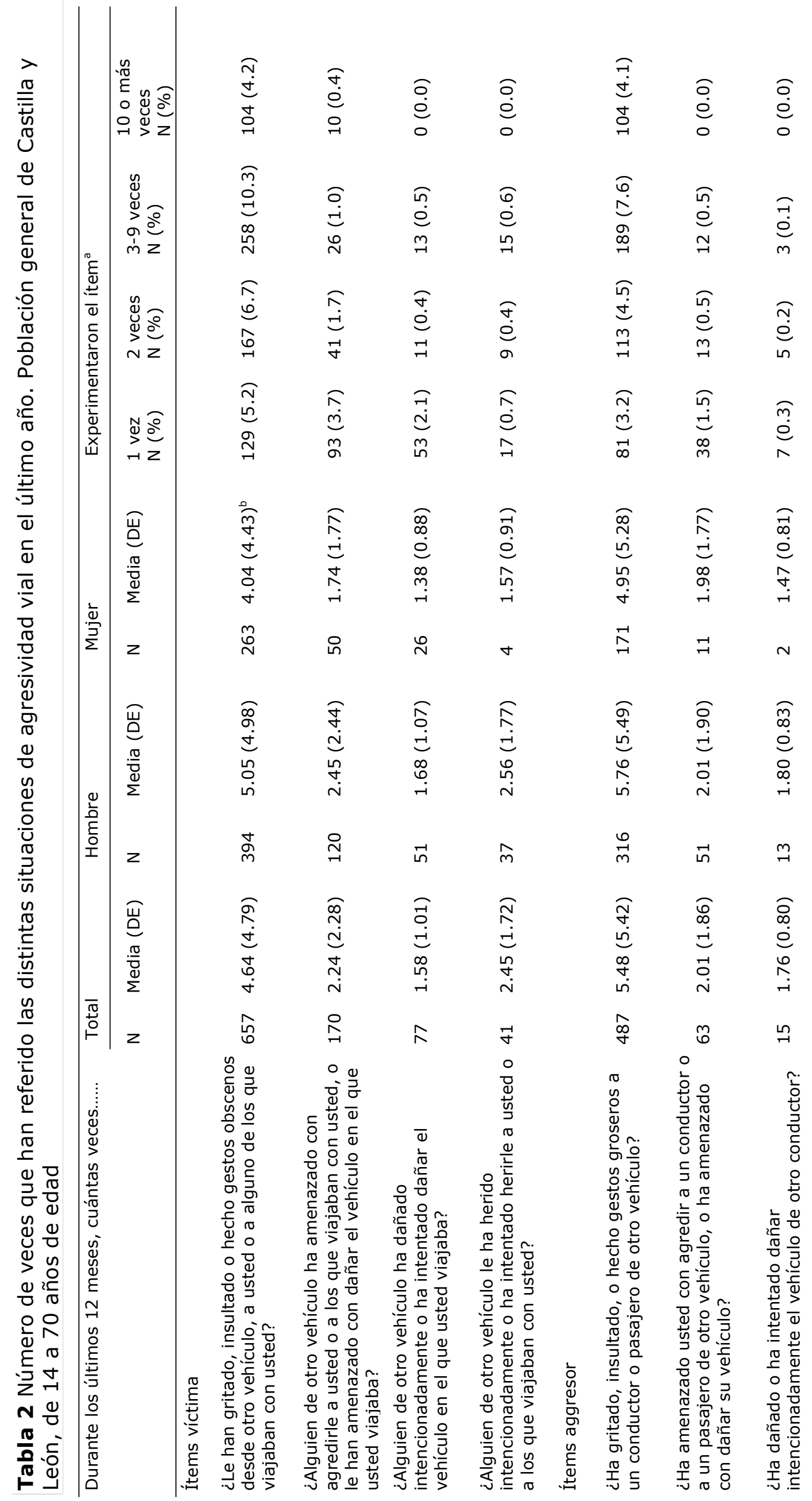




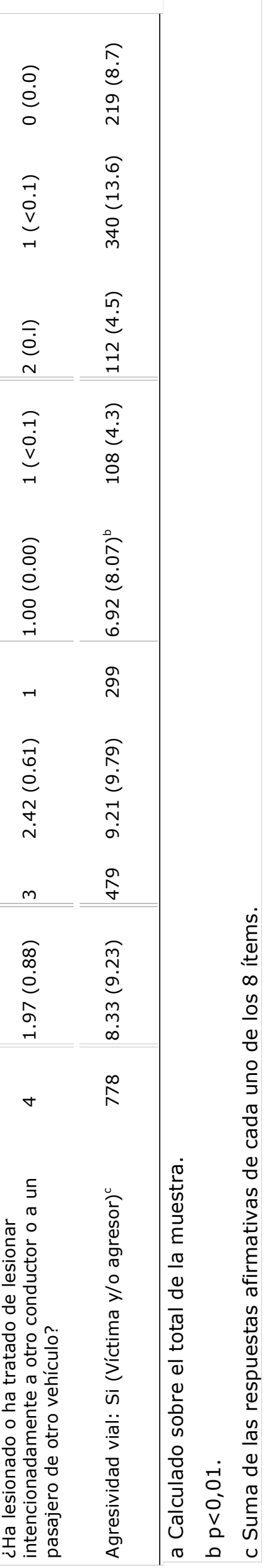




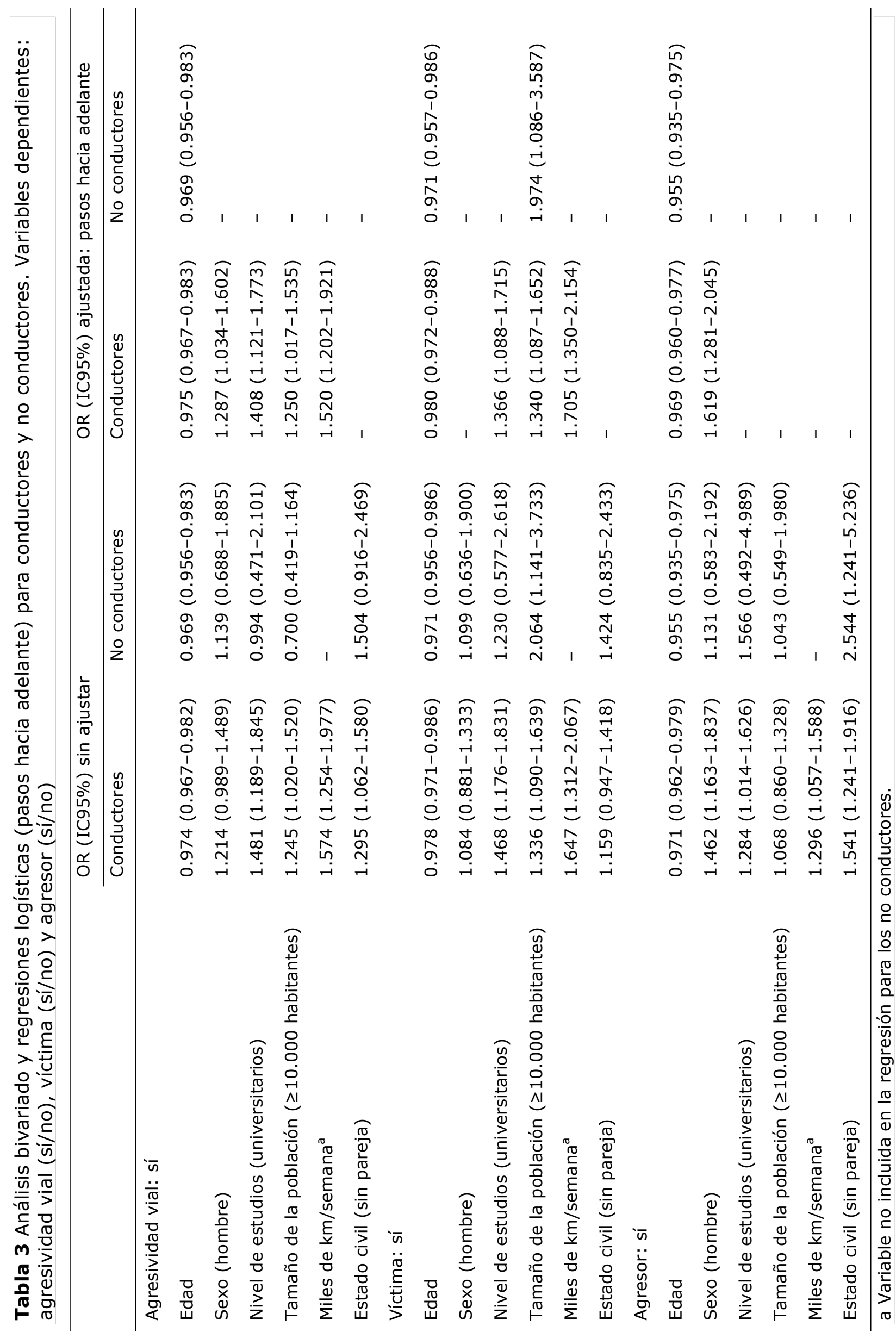




\section{Discusión}

El presente estudio muestra que la agresividad vial es frecuente en nuestra sociedad: prácticamente una de cada tres personas de la población general la refiere en el último año, y la mitad de ellos han sido tanto víctimas como agresores. Aunque la gran mayoría de los agresores viales son «leves», hay que destacar que el $2.6 \%$ de la población refirieron ser agresores viales «graves». Ciertamente, la agresividad vial es más frecuente entre los conductores, pero también se ven implicados los no conductores.

La agresividad vial se ha analizado frecuentemente en diferentes países utilizando metodologías muy diferentes [2], lo que dificulta la comparación de resultados. Varios estudios muestran, sin embargo, cifras aún más preocupantes que las observadas en el presente estudio. Según una encuesta realizada en EE.UU. [7] a 2400 conductores, en el último año el $17.0 \%$ refirió haber realizado gestos obscenos y el $46.0 \%$ haber sido víctima de ellos. Utilizando el mismo cuestionario que en el presente estudio, en Ontario (Canadá) refirieron haber sufrido agresividad vial en el último año el $47.5 \%$ en 2001 , el $46.3 \%$ en 2002 y el $40.6 \%$ en 2003 , y haber sido agresor vial el 31.0 , el 33.6 y el $33.2 \%$, respectivamente [6].

La mayoría ha experimentado varias situaciones de agresividad vial en el último año: en el presente estudio, tres de cada cuatro de los que refirieron haberla sufrido lo han hecho diez o más veces. En un estudio previo realizado en conductores, utilizando el mismo cuestionario que en este estudio, la media de veces en el último año que han referido ser agresores o víctimas de agresividad vial fue de 4.5 y 4.4 veces, respectivamente [11]. Los datos de nuestro estudio son $3.1 \pm 2.0$ y $2.8 \pm 1.3$ veces, respectivamente, si bien incluye conductores y no conductores.

La agresividad vial es más frecuente entre los hombres y entre los grupos de edad más jóvenes. En estudios previos, tener una edad más joven se ha mostrado como una variable asociada con ser tanto víctima como agresor [3,5$7,12]$. Asimismo, ser hombre se ha mostrado como un determinante para ser perpetrador, pero no víctima, de agresividad vial $[5,12]$; aunque en nuestro 
estudio los hombres tienen mayor probabilidad de ser víctima en cada uno de los indicadores de victimización, la regresión logística muestra que, al igual que en otros estudios $[5,12]$, ser hombre es un factor determinante para ser agresor. La agresividad vial se observa con más frecuencia en los que residen en áreas urbanas $[6,7]$. En el presente estudio, los que viven en localidades de más de 10000 habitantes han referido con más frecuencia casos de agresividad vial (conductores) y ser víctimas (conductores y no conductores) de agresividad vial. En estudios previos, la agresividad vial (o algunas formas de ella) es más frecuente entre aquellos con menor nivel educativo $[12,13]$. Sin embargo, en el presente estudio, experimentar agresividad vial y ser víctima de agresividad vial se ha observado más a menudo entre aquellos con estudios universitarios. Creemos que el hecho de que en la muestra analizada la proporción de conductores sea mucho mayor entre los que poseen estudios universitarios determina la mayor probabilidad de éstos de experimentar agresividad vial, a diferencia de lo que muestran otros estudios $[12,13]$.

En el presente estudio sólo se han analizado la prevalencia y los factores sociodemográficos asociados con la agresividad vial, sin considerar otros aspectos como el consumo de sustancias $[5,14]$ o las características psicológicas de los conductores (ansiedad, hostilidad, comorbilidad psiquiátrica, etc.), que en estudios anteriores se ha visto que pueden influir sobre la agresividad vial [7]. El hecho de que sólo se hayan incluido personas de hasta 70 años de edad supone una limitación, ya que hay una población de conductores mayores de 70 años que son susceptibles de sufrir agresividad vial. Otras limitaciones que debemos considerar son el hecho de que la información sea autorreportada, el sesgo de memoria (algunas de las preguntas se refieren hasta a 12 meses atrás) y que el test sobre agresividad vial no haya sido validado en la población de referencia para el estudio.

Las consecuencias reales de la agresividad vial no se conocen con exactitud. Además de la «influencia psicológica» [2] de verse sometido a ella, se ha observado un mayor riesgo de implicación en accidentes de tráfico de las personas involucradas en la agresividad vial (que se observa tanto en víctimas como agresores, y no sólo en las formas graves) [4]. Estudios previos han evidenciado que los agresores viales «graves» muestran mayores niveles de 
distrés psiquiátrico (medido con el Cuestionario General de Salud) [5]. Por otra parte, se ha señalado que la agresividad vial puede formar parte de un trastorno mental más amplio: el trastorno explosivo intermitente (DSM IV, F63.8; CIE 10, $312.34)[4,8]$.

El presente estudio muestra que la agresividad vial es común en nuestra sociedad: casi una de cada tres personas lo ha sufrido en el último año, de las cuales el $8.7 \%$ lo han experimentado diez o más veces. Éste era un aspecto del cual carecíamos de información en España [15]. Sin embargo, hay limitada información sobre qué hacer para reducir la agresividad vial y sus consecuencias. Recientemente se ha propuesto intervenir en varios campos, incluyendo acciones legales, judiciales, campañas divulgativas y cambios estructurales en nuestras carreteras [14]. Además, se ha señalado la eficacia de los programas de intervención psicológica en los conductores que provocan agresividad vial [16].

\section{Contribuciones de autoría}

F. J. Álvarez proyectó el estudio y supervisó todos los aspectos de su realización. I. Fierro y T. Gómez-Talegón analizaron los datos. Todos los autores aportaron ideas y colaboraron en la interpretación de los hallazgos, la redacción y revisión de los borradores del manuscrito. Todos los autores aprobaron la versión final del manuscrito. F. Javier Álvarez es el responsable del artículo.

\section{Conflicto de intereses}

Los autores declaran no tener ningún conflicto de intereses.

\section{Financiación}

Este estudio se ha realizado mediante un convenio de colaboración entre la Consejería de Familia e Igualdad de Oportunidades de la Junta de Castilla y León y la Universidad de Valladolid, y con la ayuda del Instituto de Salud Carlos III (Red de Trastornos Adictivos, RD06/0001/0020). 


\section{5 HIHUHQFIDV}

1. Regidor, E., Gutiérrez-Fissac, J.L., Alfaro, M., Indicadores de salud 2009. Evolución de los indicadores del estado de salud en España y su magnitud en el contexto de la Unión Europea. Madrid: Ministerio de Sanidad y Política Social; 2009.

2. Smart, R.G., Mann, R.E., 2002. Deaths injuries from road rage: cases in Canadian newspapers. CMAJ. 167, 761-762.

3. Smart, R.G., Mann, R.E., 2002. Is road rage a serious traffic problem? Traffic Inj Prev. 3, 183-189.

4. Mann, R.E., Zhao, J., Stoduto, G., 2007. Road rage and collision involvement. Am J Health Behav. 31, 384-391.

5. Smart, R.G., Asbridge, M., Mann, R.E., 2003. Psychiatric distress among road rage victims and perpetrators. Can J Psychiatry. 48, 681-688.

6. Smart, R.G., Mann, R.E., Zhao, J., 2005. Is road rage increasing? Results of a repeated survey. J Safety Res. 36, 195-201

7. Hemenway, D., Vriniotis, M., Miller, M., 2006. Is an armed society a polite society? Guns and road rage. Accid Anal Prev, 38, 687-695.

8. Álvarez, F.J., Fierro, I., 2010. Las drogas en Castilla y León, 2008. Valladolid: Consejería de Familia e Igualdad de Oportunidades, Junta de Castilla y León.

9. Álvarez, F.J., Fierro, I., Del Río M.C., 2006. Alcohol-related social consequences in Castille and Leon. Spain. Alcohol Clin Exp Res, 30, 656-664.

10. Mann, R.E., Smart, R.G., Stoduto, G., 2004. Alcohol consumption and problems among road rage victims and perpetrators. J Stud Alcohol, 65, 161-168

11. Smart, R.G., Stoduto, G., Mann, R.E., 2004. Road rage experience and behavior: vehicle, exposure, and driver factors. Traffic Inj Prev, 5, 343-348.

12. Smart, R.G., Mann, R.E., Stoduto, G., 2003. The prevalence of road rage: estimates from Ontario. Can J Public Health, 94, 247-250.

13. Roberts, L.D., Indermaur, D.W., 2008. The "homogamy" of road rage revisited. Violence Vict, 23, 758-772.

14. Asbridge, M., Smart, R.G., Mann, R.E., 2006. Can we prevent road rage?. Trauma Violence Abuse, 7, 109-121.

15. Alonso, F., Esteban, C., Calatayud, C., 2002. La agresividad en la conducción: una visión a partir de las investigaciones internacionales. Attitudes-INTRAS. 
16. Galovski, T.E., Blanchard, E.B., 2004. Road rage: a domain for psychological intervention?. Aggr Violent Behav, 9, 105-127. 

Capítulo 6

\section{Alcohol Use, Illicit Drug Use, and Road Rage}

Fierro, I., Morales, C., Álvarez, F.J.

J Stud Alcohol Drugs, 2011; 72: 185-193 



\section{Abstract}

Objective: This article examines the relationship between the consumption of alcohol and illicit drugs and the experience of road rage victimization and perpetration among drivers and nondrivers in the general population.

Method: A cross-sectional survey was designed with 2500 subjects, ages 14-70 years, living in Castile and León, Spain, of which 1276 (51\%) were males and 1224 (49\%) females. The Alcohol- Use And Drug-Use Survey of Castile and Leon, Spain 2008 focused on patterns of alcohol, tobacco, and illicit drug consumption. Potential risk factors for road-rage experience for the previous 12 months was assessed, including sociodemographics ( 7 variables), patterns of alcohol consumption ( 7 variables), and patterns of drug consumption (10 variables).

Results: Among drivers, driving under the influence of alcohol and/or cannabis during the previous year was associated with being a perpetrator of road rage (odds ratio $[\mathrm{OR}]=3.72,95 \% \mathrm{CI}[1.71-8.10]$ and 6.77 [1.55-29.48], respectively), being both a victim and perpetrator of road rage ( $O R=1.80$ [1.05-3.07] for alcohol, 5.34 [1.64-17.41] for cannabis, and 4.81 [1.09-21.16] for alcohol and cannabis), and with serious road-rage perpetration ( $O R=4.97$ [2.40-10.30] for alcohol and 17.75 [5.88-53.56] for cannabis). Problem drinking (CAGE scores $\geq 2$ ) was associated with being both a victim and perpetrator of road rage $(O R=2.74[1.67-4.50])$ and with low $(O R=1.77[1.09-2.85])$ and serious $(\mathrm{OR}=3.47[1.65-7.30])$ road-rage perpetration.

Conclusions: Driving under the influence of alcohol or cannabis and being a problem drinker are associated with the perpetration of serious road-rage behavior, as well as experiencing road-rage victimization and perpetration. 
(J. Stud. Alcohol Drugs, 72, 185-193, 2011)

Received: May 14, 2010. Revision: October 8, 2010.

This work was supported by grants from the Consejería de Familia e Igualdad de Oportunidades and from the Ministerio de Sanidad y Consumo, Redes Temáticas de Investigación Cooperativa, Red de Trastornos Adictivos (RD06/0001/0020). 


\section{Introduction}

Although there is no established definition of road rage at a scientific level $[1,2]$, it can be understood as "an attempt to intimidate, threaten, injure, or kill other drivers, passengers, or pedestrians" [2, p. 195]. There is epidemiological evidence that victims and perpetrators of road rage, as well as serious road-rage perpetrators-those who intentionally damage or attempt to damage another driver's car or injure or attempt to injure the driver or passenger of another vehicle-have a significantly higher risk of becoming involved in traffic accidents [3]. However, few studies exist about the causes or risk factors of having an experience of road rage, although it has been identified as an emerging problem in several countries such as Australia [4], Canada [2], the United Kingdom [5], and the United States [6], and has been a popular issue in the media.

It has been broadly documented that heavy drinkers and consumers of illicit drugs are often victims or perpetrators of aggression, as well as being "at fault" in traffic crashes [7-12] and that cannabis users are frequently observed to be drivers or people injured or killed in traffic accidents [13-15]. However, there are few studies on how the consumption of alcohol and other illicit substances are related to the experience of road rage. Only four such studies have been published.

Mann et al. [16], with a sample of 2610 adult residents in Ontario, Canada, ages 18 years or older, found that there was a significant relationship between the problem drinking of alcohol-measured by the Alcohol Use Identification Test (AUDIT)-and the experience of road-rage victimization and perpetration.

Likewise, two studies conducted with 2421 adults living in Ontario, Canada, concluded that cannabis, cocaine, or Ecstasy use during the previous year and/or problem drinking of alcohol were significantly higher among those involved in most serious road-rage behaviors [17], and that cannabis use was associated with the experience of victimization and perpetration of road rage, whereas the use of stimulants significantly increased the likelihood of victimization and being classified as a serious road-rage perpetrator [18]. 
Finally, Yu et al. [19] studied aggressive driving and road rage in a sample of 431 patients in treatment for alcoholism in New York State. All subjects were undergoing alcoholism treatment because of a drinking and driving-related reason. The results demonstrated that aggressive driving and road rage were two separate behaviors that simultaneously influenced each other, and both tended to be more affected by alcohol problems-measured with the Diagnostic and Statistical Manual of Mental Disorders, Fourth Edition [20]- and other variables that indicate problem behaviors (such as driving in an inadequate way and depression) rather than the frequency of alcohol use, driving after drinking, and experiencing stressful events.

In Europe, there is a lack of studies on consumption of substances and their effect on road-rage behaviors. The realization of studies at an international level is important both to determine the magnitude of the problem and to carry out comparisons among samples from different countries with the purpose of establishing firm conclusions about the consumption of substances and other factors associated with road rage [1]. Likewise, it is necessary to compare the effect of alcohol consumption with other drugs, determining the extent to which consumers of alcohol and other substances differ in terms of being involved in road-rage behaviors [19].

There are several questionnaires designed to measure the construct of road rage. Among those most used are the Propensity for Angry Driving Scale [21], used to identify drivers more likely to get angry with others while driving and consequently to participate in hostile driving behaviors or acts of road rage; the Driving Anger Scale [22], which measures the trait of driving with anger or propensity to experience rage while driving; the Driving Anger Expression Inventory [23], which measures the usual way of expressing anger while driving; and last, the indicators from a taxonomy of road-rage behavior developed by Smart et al. $[2,24]$, which quantify the frequency of involvement in acts of road rage as victims and as perpetrators during the last 12 months. In our study, we used these last indicators because the questionnaire has only eight items, making it easy to administer and include in population surveys. It also allows comparison of the results obtained with most previous studies on road rage and 
substance use [16-18] as it has been the most widely used measurement instrument.

The objective of this study is to analyze the association of alcohol and illicit drug consumption on road rage victimization and perpetration in both drivers and nondrivers. The study used a representative sample of the population from Castile and León, in Spain. We included nondrivers in the study because both drivers and nondrivers (i.e., a passenger in a car when the driver engages in road-rage behavior) could be involved in road-rage behavior, either as victims and/or perpetrators.

\section{Methods}

This study was approved by the Comité Ético de Investigación Clínica, Facultad de Medicina de la Universidad de Valladolid, Reference number 2008/18.

\section{Sample and fieldwork procedure}

Data was collected using the Alcohol-Use and Drug-Use Survey of Castile and León, Spain 2008 and through face-to-face interviews from April 21-May 22, 2008 [25]. The survey, which has been conducted regularly since the late 1980s, focuses on patterns of alcohol, tobacco, and illicit drug consumption in the general population. In its most recent version (2008), questions regarding road rage were addressed for the first time.

The participants were selected at random from a representative sample of Castile and León households that represent 2528417 inhabitants, consisting of 1251082 males and 1277335 females. The sample was taken from the population register data of 2007 [26]. People from 14 to 70 years of age living in Castile and León, Spain, were the target population.

A final sample of 2500 valid interviews was selected. The sample selection was stratified as follows: first, proportionally according to the number of inhabitants in the communities (using five ranges of size of the population: <10000, 1000049999, 50000-99999, 100000-249999, >250000); second, by province on the 
basis of the regional administrative division ( 9 provinces); third, by age group; and fourth, by gender, as in previous surveys with the same target population [27]. If the selected person refused to take part in the study, a new person of the same gender and age range was chosen. Information from 16 surveys was withdrawn because of missing information or incoherence in the responses, and a new person of the same gender and age range was selected. Quality control of the response was done in 414 of the surveys by telephone interview. The field trial and quality control was carried out by the company Telecyl (Valladolid, Spain; www.telecyl.com).

A total of 2500 subjects participated in the study, of which $51.0 \%(n=1276)$ were males and $49.0 \%(n=1224)$ females. Of the sample $63.6 \%(n=1591)$ drove vehicles, 36.4\% $(n=909)$ did not drive. The age distribution of the sample was as follows: $14-19$ years $=7.6 \%(n=189), 20-29$ years $=17.9 \%(n$ $=447), 30-39$ years $=20.9 \%(n=522), 40-49$ years $=21.2 \%(n=530), 50-59$ years $=17.0 \%(n=426), 60-70$ years $=15.4 \%(n=386)$. The sociodemographic characteristics of the sample are shown in Table 1.

\section{Outcome measures and risk factors}

Road-rage experience and behavior for the previous 12 months was measured using eight questions: four items on road-rage victimization and four items on road-rage perpetration $[2,16,17]$. The victimization items measured how many times, during the past 12 months, someone in another other vehicle had (a) shouted, cursed, or made rude gestures at you or others with you; (b) threatened to hurt you or others with you or threatened to damage the vehicle you were in; (c) intentionally damaged or attempted to damage the vehicle you were in; or (d) intentionally hurt or attempted to hurt you or others with you. The perpetration items measured how many times, during the past 12 months, the respondent had (a) shouted, cursed, or made rude gestures at a driver or passenger in another vehicle; (b) threatened to hurt a driver or passenger in another vehicle or threatened to damage their vehicle; (c) intentionally damaged or attempted to damage another driver's vehicle; or (d) intentionally hurt or attempted to hurt a driver or passenger in another vehicle. 
The eight items show an acceptable reliability: $a=0.674$ [28]. In an inter item correlation analysis of the four perpetration items, with a sample of aggressors only, there was a negative correlation of the first item with the second $(r=-.10)$ and the third ( $r=-.87)$ and a positive correlation among items 2 and $3(r=.25)$. Therefore, the perpetrators' items 2, 3, and 4 were collapsed as "serious" roadrage perpetration. (Although there were no correlations with the fourth item because there was a lack of answers to it in the aggressors only sample, the question can be considered as serious perpetration.)

The road-rage outcome was established in different categories:

(a) no road-rage experience, (b) victim of road rage only, (c) perpetrator of road rage only, and (d) being either victim or a perpetrator of road rage. Likewise, the road-rage perpetrator experience was divided in two categories: low perpetrator, which consisted of responding positively to the first perpetration item only, and serious perpetrator, which was based on a positive response to at least one of the last three perpetration items.

Road-rage experience was assessed among both drivers and nondrivers. The latter have been included in the study because nondrivers could be passengers in a car when the driver engages in road-rage behavior. Furthermore, note the way in which the eight questions that refer to road rage are formulated: None of them specifies that the person being interviewed was necessarily driving at the time road rage was observed, nor is it specified that the person who suffered or perpetrated the aggression was at that particular time the driver $[2,16,17]$. The questionnaire to evaluate road rage in this study has already been used in other studies with samples from the general population, not just drivers $[2,16,17]$. Potential risk factors for road rage were classified into three groups (Table 1 ): (a) sociodemographics ( 7 variables), (b) patterns of alcohol consumption (7 variables), and (c) patterns of drug consumption (10 variables). Regarding sociodemographics, these were dichotomized variables with more than two categories. The variables considered were (a) gender, (b) age (as a continuous variable), (c) civil status (married or with couple/others), (d) education level (basic studies or no studies/higher than basic studies), (e) driver (si/no; driver was considered those with a valid driving license and those who drove at least 1 day in the previous year), (f) population size of community 10000 or more 


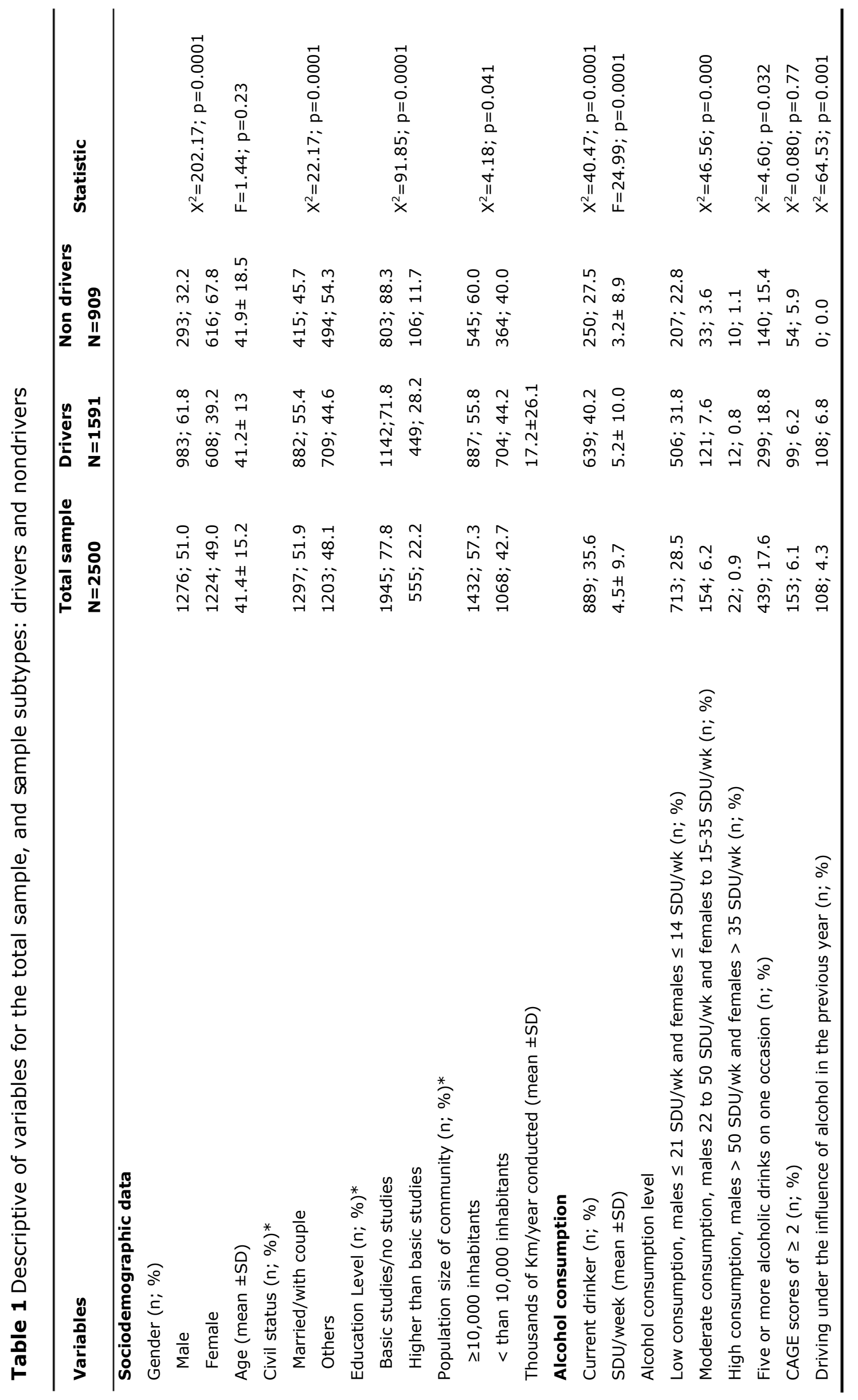




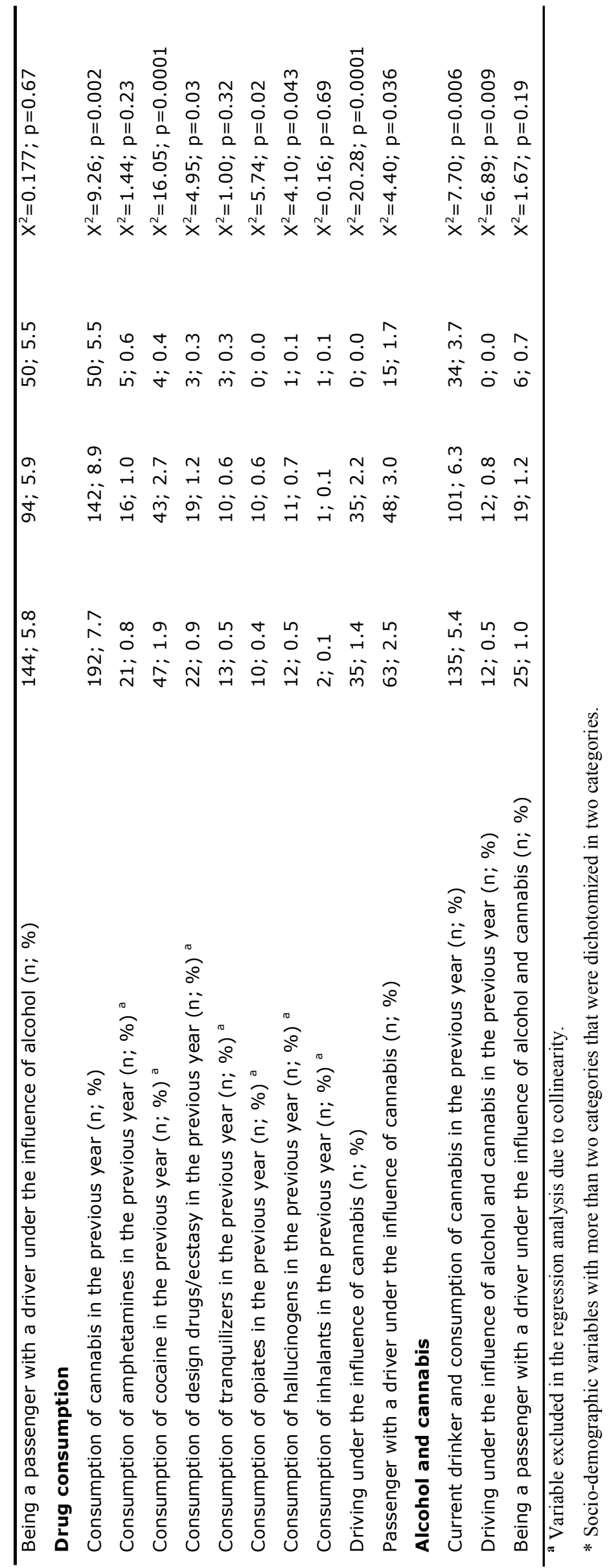


inhabitants ( $\mathrm{si} / \mathrm{no}$ ), and $(\mathrm{g})$ thousands of kilometers per year driven (as a continuous variable and only considered for drivers).

Regarding patterns of alcohol consumption variables, the following were considered:

(a) Current drinker (si/no). This was defined as those who have had at least one drink per week during the past year.

(b) Alcohol consumption in standard drink unit (SDU): SDUs per week (mean). Current drinkers were split into weekly and occasional drinkers. Weekly drinkers were those who had drunk alcohol at least once a week over the preceding year. Occasional drinkers were those who had drunk alcohol less than once a week over the preceding year. The survey assessed drinkers for alcohol intake using a retrospective weekly recall for those who drank at least once a week (weekly drinkers). For those who drank less frequently than once a week (occasional drinkers), a quantity/frequency approach was used. The amount of alcohol was expressed either in grams of absolute alcohol ingested daily or in SDUs, based on the alcohol content of Spanish drinks and the intake level of each of the different beverages [27]. A Spanish SDU was set at $10 \mathrm{~g}$ of pure ethanol [29].

(c) Alcohol consumption level. Drinkers were classified as having consumption levels as follows: low consumption $=21$ or fewer SDUs per week for males and 14 or fewer for females; moderate consumption $=22-50$ SDUs per week for males and 15-35 for females; high consumption = more than 50 SDUs per week for males and more than 35 for females [27].

(d) To have consumed five or more alcoholic drinks in one occasion during the previous 30 days (si/no).

(e) CAGE scores of 2 or greater (si/no). The CAGE questionnaire, in the Spanish validated version [30], was used with current drinkers, and we consider a score of 2 or more as defining a "problem drinker" [31].

(f) Driving under the influence of alcohol in the previous year (si/no). This was considered for drivers.

(g) Being a passenger with a driver under the influence of alcohol (si/no). This was considered for nondrivers.

Related to other illicit drugs, the variables were the following: 
(a) To have consumed cannabis, amphetamines, cocaine, designer drugs (e.g., Ecstasy), tranquilizers, opiates, hallucinogens, and inhalants in the previous year (si/no).

(b) Being a passenger with a driver under the infl uence of cannabis (si/no). This was considered for nondrivers.

(c) Driving under the influence of cannabis in the previous year (si/no). This was considered for drivers.

The following interaction factors were assessed:

(a) Current Drinker (si/no) $\times$ To Have Consumed Cannabis in the Previous Year (si/no). This was considered for drivers and nondrivers.

(b) Driving Under the Influence of Alcohol (si/no) $\times$ Driving Under the Influence of Cannabis (si/no). This was considered for drivers.

(c) Being a Passenger With a Driver Under the Influence of Alcohol (si/no) $\times$ Being a Passenger With a Driver Under the Influence of Cannabis (si/no). This was considered for nondrivers.

\section{Statistical analysis}

The statistical analysis was performed using SPSS version 15.0 (SPSS Inc., Chicago, IL).

To assess risk factors for road rage, we used one-way analysis of variance for univariate continuous variables and the chi-square test for categorical variables. In addition, we conducted Fisher's exact test whenever the expected value of at least one cell of the chi-square was less than 5.

Because of the large number $(n=24)$ of potential risk factors, we avoided multicollinearity among the explanatory variables by performing collinearity diagnostic analyses [32,33]. We performed the stepwise selection of variables from the models with the following criteria: tolerance greater than 0.4 or variance inflation less than 2.5 , condition number less than 10 , and a variance of two or more variables no greater than 0.5 . The variables that were excluded because of collinearity are identified in Table 1. 
Multinomial logistic regression analysis with forward step was carried out separately for drivers and nondrivers. In a first analysis the dependent variable was type of road rage (none, only victim, only perpetrator, and either victim and perpetrator). In a second analysis, the dependent variable was type of road-rage perpetration (none, low perpetration, and serious perpetration). For all models, the adjusted odds ratio (OR) indicated the association of interest. OR and $95 \%$ confidence interval (CI) are given. A $p$ value of .05 or less was considered significant.

\section{Results}

\section{Road rage}

Regarding the road-rage experience, among drivers, $55.6 \%(n=885)$ had not had an experience of road rage, $15.3 \%(n=243)$ were victims only, $5.0 \%(n=$ 80) were perpetrators only, and $24.1 \%(n=383)$ were both victims and perpetrators. And finally, among the nondrivers, 92.1\% $(n=836)$ had not had an experience of road rage, $3.5 \%(n=32)$ were victims only, $1.2 \%(n=11)$ were perpetrators only, and 3.2\% $(n=29)$ were both victims and perpetrators. Considering the different levels of road-rage perpetration, among drivers, $25.2 \%$ $(n=400)$ were low-level perpetrators and 3.9\% $(n=62)$ were serious perpetrators; and, among nondrivers, $4.3 \%(n=39)$ were low perpetrators and $0.2 \%(n=2)$ were serious perpetrators.

\section{Road-rage experience and risk factors}

In Tables 2 and 3, the variables are presented that, in the regression model, were significantly associated with being a victim and/or perpetrator of road rage (Table 2) and with the level of road-rage perpetration (Table 3), for drivers and nondrivers (passengers), respectively.

Being only a victim of road rage was associated with drivers from communities with 10000 or more inhabitants $(O R=1.78)$, with a higher education $(O R=$ 1.53), and with increased thousands of kilometers driven per year $(O R=1.01)$. Among nondrivers, no variables were associated with being a victim only. 
Being only a road-rage perpetrator was associated with male drivers (OR = $2.28)$, as well as with driving under the influence of cannabis $(O R=6.77)$ or alcohol $(O R=3.72)$, and the association decreased with age $(O R=0.97)$. Among nondrivers, being a passenger of a driver under the influence of alcohol was associated with road-rage perpetration only $(O R=8.71)$.

Being both victim and perpetrator of road rage was associated with drivers with a higher education $(O R=1.40)$, increases in the thousands of kilometers driven per year $(O R=1.009)$, drivers who had driven under the influence of alcohol $(O R=1.80)$ or cannabis $(O R=5.34)$ or alcohol and cannabis $(O R=4.81)$, and problem drinkers $(O R=2.74)$, and it decreased with age $(O R=0.97)$. Among nondrivers, being both victim and perpetrator of road rage was associated with being a passenger of a driver under the influence of alcohol $(O R=5.31)$, cannabis $(O R=6.53)$, or alcohol and cannabis $(O R=11.90)$, and the risk decreased with age $(O R=0.96)$.

Being a low perpetrator was associated with male drivers $(O R=1.36)$ and with problem drinkers $(O R=1.77)$, whereas the association decreased with older driver age $(O R=0.98)$. Among nondrivers, low perpetration was associated with being a passenger of a driver under the influence of alcohol $(O R=6.41)$, of cannabis $(O R=5.73)$, or of alcohol and cannabis $(O R=8.62)$, and the risk decreased with age $(O R=0.96)$.

Being a serious perpetrator was associated with male drivers $(O R=2.49)$, drivers who during the previous year had driven under the influence of alcohol $(O R=4.97)$ or cannabis $(O R=17.75)$, and problem drinkers $(O R=3.47)$. Older drivers were associated with a significantly smaller likelihood of being a serious perpetrator of road rage $(O R=0.95)$. Among nondrivers, no variables were associated with serious road-rage perpetration. 


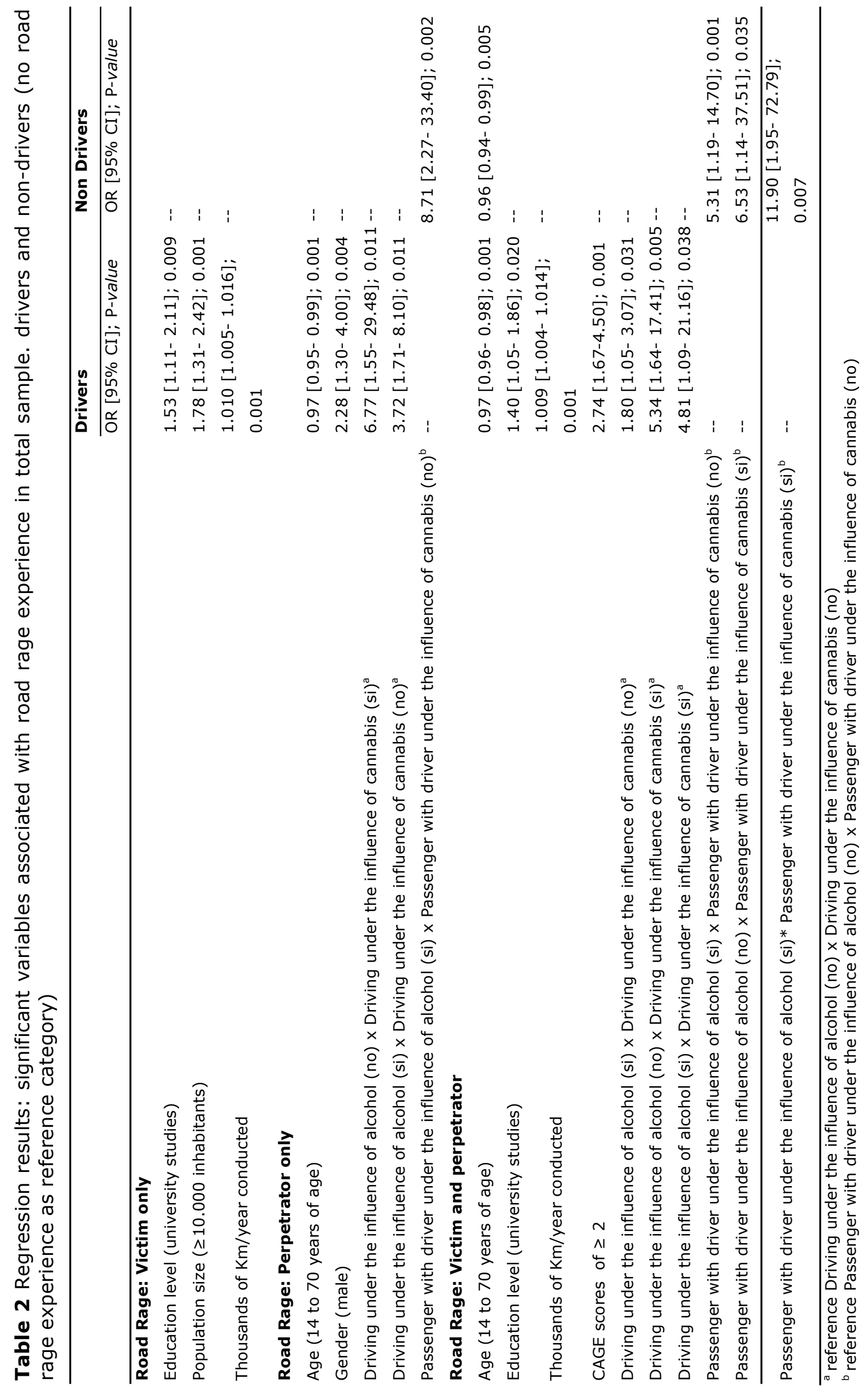




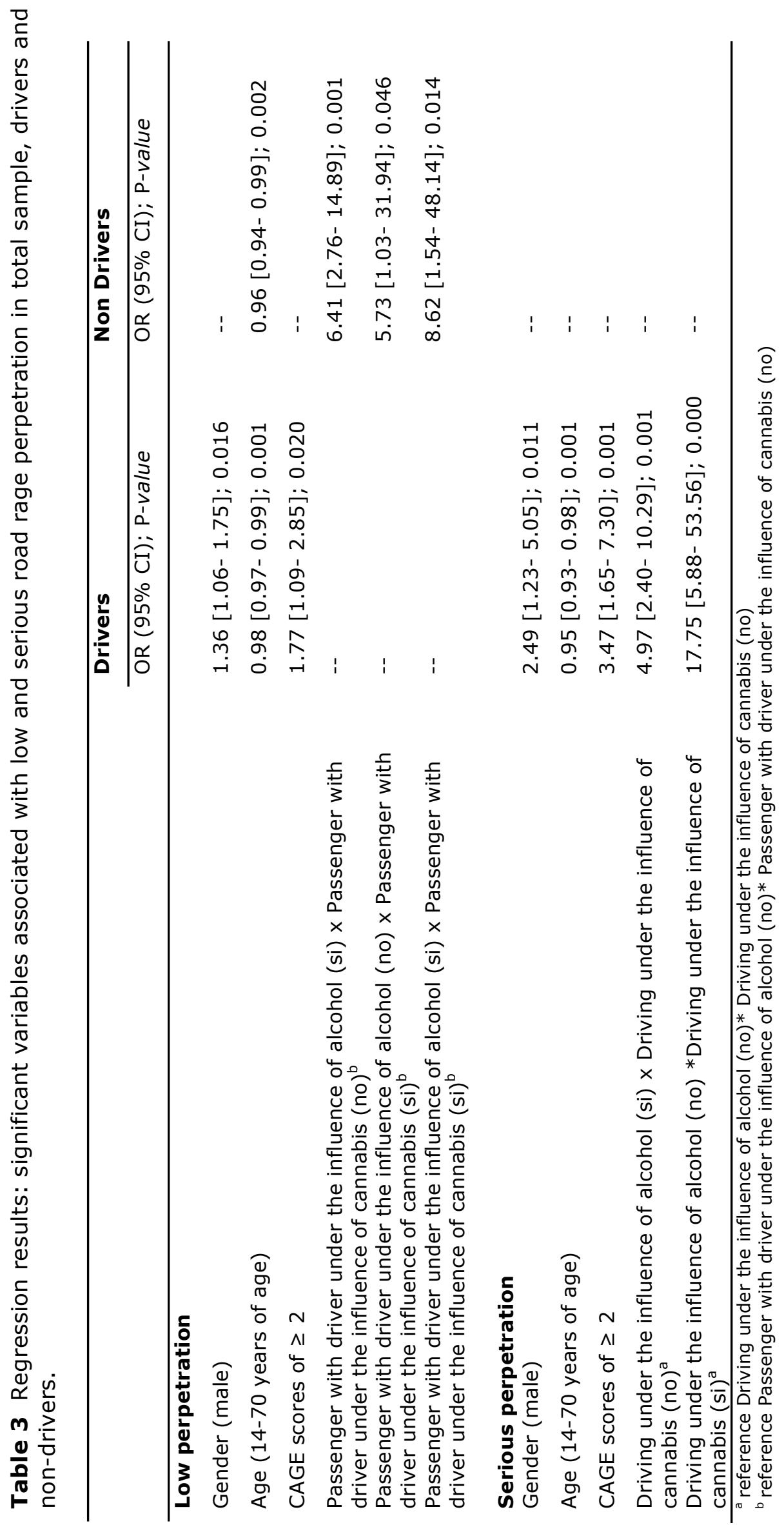




\section{Discussion}

Road rage is a frequent phenomenon: more people are likely to be both victims and perpetrators, and most are low-level perpetrators. The present study shows that driving under the influence of alcohol and/or cannabis and being a "problem drinker" (CAGE scores $\geq 2$ ) are associated with serious road-rage perpetration and with the highest prevalence of experiences of road-rage victimization and perpetration. Among nondrivers, being the passenger of a driver under the influence of alcohol and/or cannabis increases the probability of being both victim and perpetrator, and a low perpetrator of road rage.

As combined use of substances is frequent, in the current study we have assessed the effect of the interaction of alcohol and cannabis use (Current Drinker [si/no] $\times$ To Have Consumed Cannabis in the Previous Year [si/no]). The results show that alcohol and/or cannabis use was related to road rage. Furthermore, it is well known that combined use of substances (drug/drug or alcohol/drug) leads to an increased risk for road traffic involvement $[13,34]$.

In our study, none of the variables related to patterns of alcohol consumption (such as frequency or quantity) were associated with road rage, whereas certain behaviors related to drinking-like driving under the influence of alcohol or being a passenger of a driver under the influence of alcohol-were associated with road rage. It is noteworthy that heavy volume drinking and even consumption of five or more drinks were not associated with road rage unless the respondents reported driving under the influence of alcohol (or riding as a passenger with a driver under the influence). Our findings regarding alcohol consumption are consistent with the results of previous studies in this area, although the comparison of results should be carried out with caution because different variables or factors associated with road rage were used. A significant relationship has been noted between the subscale of problematic alcohol consumption of the AUDIT questionnaire and the experience of victimization and perpetration in road-rage incidents [16], drivers with drinking problems as measured by the AUDIT are significantly more likely to be serious road-rage perpetrators [17], and a significant association exists between measures of road 
rage (angry/threatening driving) and driving above the blood alcohol concentration limit in the previous year [35].

Butters et al. $[17,18]$ found that consumption of cannabis during the previous year was associated with road rage victimization and perpetration, and with the most serious forms of road-rage perpetration. Our study also found that those who had driven under the influence of cannabis were more likely to be perpetrators and victims of road rage, to be perpetrators only, and to be serious perpetrators. It is necessary to explore the mechanisms by which this illegal drug is related to road rage, as pointed out by other authors [17]. Although it is known that alcohol has disinhibitory effects, thus contributing to aggressive behavior, cannabis is rarely associated with aggression; on the contrary, it tends to produce calm and passivity [36]. It has been documented that cannabisdependent subjects who report problems controlling their violent behavior while intoxicated tend to react with aggression [37]; chronic cannabis users (equivalent to smoking daily for approximately 14 years) tend to demonstrate higher levels of aggressive behavior during the first week of abstinence, by which we can understand aggressive behavior as an additional component in the withdrawal symptoms of cannabis [38]. However, our sample was composed mainly of recreational cannabis users rather than dependent patient or long-term daily cannabis users. In a recent study, driving under the influence of cannabis was associated with risky driving (careless style of driving) but not with aggressive driving [39]. Furthermore, sensation seeking and impulsivity were found to be predictors of driving under the influence of cannabis [39].

Our data are also consistent with previous studies that postulated that the use of alcohol and illicit drugs is more strongly associated with road-rage perpetration than with victimization [17]. In our study, we found no variables regarding the consumption of alcohol or illicit drugs associated with the experience of being only a victim of road rage. Among the sociodemographic variables, the age variable is particularly noteworthy because it was the only variable that was significantly associated with all the types of road rage experience: At younger ages, subjects were more likely to experience road rage in general. Being young has been documented previously as a risk variable in being both perpetrator and victim of road rage $[16,17,40]$. Another aspect that stands out is gender: Being a 
man has been identified as a crucial variable in being a perpetrator (but not a victim) of road rage $[16,17,24,41]$. This was also demonstrated by our results, where there were no significant differences by gender in those who were victims of road rage only. In our study, having a higher educational level was associated with both being a victim of road rage only and being both victim and perpetrator. In previous studies, road rage, or some form of road rage such as threatening someone, is more frequent among those whose educational level is lower [42]. In our opinion, the fact that in our sample the proportion of drivers with university studies is much greater determines the greater probability of those drivers experiencing road rage, compared with other studies [42]. In addition, we have used educational level as a dichotomized variable (basic studies or no studies vs. higher than basic studies), whereas in other studies several categories were used [42]. Furthermore, however, the existence of cultural differences between studies (Canadian population vs. Spanish population), for instance, cannot be ignored.

Our results agree with studies that show that a greater number of people living in towns or driving in urban areas leads to a greater likelihood of experiencing road rage $[16,41,42]$. Some authors refer to the fact that victimization and aggression are significantly higher in drivers as the number of kilometers driven increases, perhaps because drivers with a lot of experience in the vehicle may experience more frustration when they fail to use their greater performance capacity on congested urban roads [24]. In our study, there was a significantly greater probability of experiencing victimization in municipalities with 10,000 or more inhabitants and as the kilometers driven per year increased.

Among the limitations of our study was its cross-sectional design, which did not allow us to prove conclusively that the use of illicit drugs or alcohol directly affects or causes road-rage behavior. Likewise, the association of alcohol consumption in conjunction with other illegal substances such as cocaine and amphetamines (among others) was not included in the analysis. Finally, other psychological characteristics of drivers that have been shown in previous studies to be associated with the victimization and perpetration of road rage were not considered in the analysis, such as anxiety, hostility, anger, mental stress, 
psychiatric morbidity, personality traits and specific measures such as competitiveness during driving, and "driving anger," among others [17,42-45].

Driving under the influence of substances (alcohol, drugs) is frequent, and, with recent concern about cannabis, has been recognized as a risk for road traffic accidents [46-48], with intervention to reduce drug-related crashes being seen as a priority [49-51]. Furthermore, as shown in our study, road rage is a frequent phenomenon that requires attention and is associated with certain behaviors related with the consumption of alcohol and drugs. The findings from the present study have implications for the development of appropriate targeted interventions aimed at reducing risky driving practices. 


\section{References}

1. Smart, R.G., \& Mann, R.E., 2002. Is road rage a serious traffic problem? Traffic Inj Prev. 3, 183-189.

2. Smart, R.G., Mann, R. E., Zhao, J., \& Stoduto, G., 2005. Is road rage increasing? Results of a repeated survey. J Safety Res. 36, 195-201.

3. Mann, R.E., Zhao, J., Stoduto, G., Adlaf, E.M., Smart, R.G., Donovan, J. E., 2007. Road rage and collision involvement. Am J Health Behav. 31, 384-391.

4. Harding, R.W., Morgan, F. H., Indermaur, D., Ferrante, A. M., Blagg, H., 1998. Road rage and the epidemiology of violence: Something old, something new. Studies on Crime and Crime Prevention. 7, 221-238.

5. Joint, M., 1995. Road rage. Washington, DC: AAA Foundation for Traffic Safety.

6. Batten, P.J., Penn, D.W., Bloom, J.D., 2000. A 36-year history of fatal road rage in Marion County, Oregon: 1963-1998. J Forensic Sci. 45, 397-399.

7. Chipman, M.L., Macdonald, S., Mann, R. E., 2003. Being "at fault" in traffic crashes: Does alcohol, cannabis, cocaine, or polydrug abuse make a difference? Inj Prev. 9, 343-348.

8. Grisso, J.A., Schwarz, D.F., Hirschinger, N., Sammel, M., Brensinger, C.,Santanna, J., Teeple, L., 1999. Violent injuries among women in an urban area. N Engl J Med. 341, 1899-1905.

9. Harrison, L.D., Erickson, P.G., Adlaf, E., Freeman, C., 2001. The drugs-violence nexus among American and Canadian youth. Subst Use Misuse. 36, 2065-2086.

10. Macdonald, S., Anglin-Bodrug, K., Mann, R.E., Erickson, P., Hathaway, A., Chipman, M., Rylett, M., 2003. Injury risk associated with cannabis and cocaine use. Drug Alcohol Depend. 72, 99-115.

11. Smart, R.G., Mann, R.E., Tyson, L. A., 1997. Drugs and violence among Ontario students. J Psychoactive Drugs. 29, 369-373.

12. Wells, S., Graham, K., West, P., 2000. Alcohol-related aggression in the general population. J of Stud Alcohol. 61, 626-632.

13. Drummer, O.H., Gerostamoulos, J., Batziris, H., Chu, M., Caplehorn, J., Robertson, M. D., Swann, P., 2004. The involvement of drugs in drivers of motor vehicles killed in Australian road traffi c crashes. Accid Anal Prev. 36, 239-248. 
14. Laumon, B., Gadegbeku, B., Martin, J.L., Biecheler, M.B., the SAM Group., 2005. Cannabis intoxication and fatal road crashes in France: Population based casecontrol study. BMJ. 331, 1371-1376.

15. Ramaekers, J., Berghaus, G., van Laar, M., Drummer, O. 2004. Dose related risk of motor vehicle crashes after cannabis use. Drug Alcohol Depend. 73, 109-119.

16. Mann, R.E., Smart, R. G., Stoduto, G., Adlaf, E.M., Ialomiteanu, A., 2004. Alcohol consumption and problems among road rage victims and perpetrators. J Stud Alcohol. 65, 161-168.

17. Butters, J.E., Smart, R. G., Mann, R.E., Asbridge, M., 2005. Illicit drug use, alcohol use and problem drinking among infrequent and frequent road ragers. Drug Alcohol Depend. 80, 169-175.

18. Butters, J.E., Mann, R.E., Smart, R.G., 2006. Assessing road rage victimization and perpetration in the Ontario adult population: The impact of illicit drug use and psychiatric distress. Can J Public Health. 97, 96-99.

19. Yu, J., Evans, P., Perfetti, L., 2004. Road aggression among drinking drivers: Alcohol and non-alcohol effects on aggressive driving and road rage. J Crim Justice. 32, 421430.

20. American Psychiatric Association. 1994. Diagnostic and statistical manual of mental disorders (4th ed.). Washington, DC: Author.

21. DePasquale, J.P., Geller, E. S., Clarke, S.W., Littleton, L.C., 2001. Measuring road rage: Development of the Propensity for Angry Driving Scale. J Safety Res. 32, 1-16.

22. Deffenbacher, J.L., Oetting, E.R., Lynch, R.S., 1994. Development of a driving anger scale. Psychological Reports. 74, 83-91.

23. Deffenbacher, J.L., Lynch, R. S., Oetting, E.R., Swaim, R. C., 2002. The driving anger expression inventory: A measure of how people express their anger on the road. Behav Res Ther. 40, 717-737.

24. Smart, R G., Stoduto, G., Mann, R. E., Adlaf, E.M., 2004. Road rage experience and behavior: Vehicle, exposure, and driver factors. Traffic Inj Prev. 5, 343-348.

25. Álvarez, F., Fierro, I., 2010. El consumo de drogas en Castilla y León, 2008. Valladolid, Spain: Junta de Castilla y León, Consejería de Familia e Igualdad de Oportunidades.

26. Instituto Nacional de Estadística. 2008. Padrón municipal. Madrid, Spain: Author. Retrieved from: http://www.ine.es 
27. Álvarez, F.J., Fierro, I., Del Río, M.C., 2006. Alcohol-related social consequences in Castile and León, Spain. Alcoholism: Clinical and Experimental Research. 30, 656664.

28. George, D., Mallery, P., 1994. SPSS/PC + step by step: A simple guide and reference. Florence, KY: Wadsworth Publishing (Cengage Learning).

29. Gual, A., Martos, A.R., Lligoña, A., Llopis, J.J., 1999. Does the concept of a standard drink apply to viticultural societies? Alcohol Alcohol. 34, 153-160.

30. Rodríguez-Martos, A., Navarro, R., Vecino, C., Pérez, R., 1986. Validación de los cuestionarios KFA (CBA) y CAGE para diagnóstico del alcoholismo. Drogalcohol. 11, 132-139.

31. Álvarez, F.J., Del Rio, M.C., 1994. Screening for problem drinkers in a general population survey in Spain by use of the CAGE scale. J of Stud Alcohol. 55, 471-474.

32. Belsley, D.A., Kuh, E., Welsch, R.E., 1980. Regression diagnostics: Identifying influential data and sources of collinearity. Hoboken, N.J.: John Wiley and Sons.

33. Kleinbaum, D., Kupper, L., Muller, K., Nizam, A., 1998. Applied regression analysis and other multivariable methods. Pacific Grove, CA: Duxbury Press.

34. Mathijssen, R., Houwing, S., 2005. The prevalence and relative risk of drink and drug driving in the Netherlands: a case-control study in the Tilburg pólice district. Leidschendam: SWOV. http://www.swov.nl/rapport/r-2005-09.pdf (accesses 16.06.14).

35. Wells-Parker, E., Ceminsky, J., Hallberg, V., Snow, R. W., Dunaway, G., Guiling, S., Anderson, B., 2002. An exploratory study of the relationship between road rage and crash experience in a representative sample of US drivers. Accid Anal Prev. 34, 271278.

36. Pliner, P., Cappell, H., Miles, G., 1972. Observer judgments of intoxicated behavior during social interaction: A comparison of alcohol and marijuana. New York: Futura Publishing.

37. Arendt, M., Rosenberg, R., Fjordback, L., Brandholdt, J., Foldager, L., Sher, L., MunkJørgensen, P., 2007. Testing the self-medication hypothesis of depression and aggression in cannabis-dependent subjects. Psychological Medicine. 37, 935-945.

38. Kouri, E.M., Pope, H.G., Jr., Lukas, S.E., 1999. Changes in aggressive behavior during withdrawal from long-term marijuana use. Psychopharmacology. 143, 302308. 
39. Richer, I., Bergeron, J., 2009. Driving under the influence of cannabis: Links with dangerous driving, psychological predictors, and accident involvement. Accid Anal Prev. 41, 299-307.

40. Smart, R.G., Asbridge, M., Mann, R.E., Adlaf, E. M., 2003. Psychiatric distress among road rage victims and perpetrators. Can J Psychiatry. 48, 681-688.

41. Asbridge, M., Smart, R.G., Mann, R.E., 2003. The "homogamy" of road rage: Understanding the relationship between victimization and offending among aggressive and violent motorists. Violence and Victims. 18, 517-531.

42. Smart, R., Mann, R.E., Stoduto, G., 2003. The prevalence of road rage: Estimates from Ontario. Can J Public Health. 94, 247-250.

43. Fong, G., Frost, D., Stansfeld, S., 2001. Road rage: A psychiatric phenomenon? Soc Psychiatry Psychiatr Epidemiol. 36, 277-286.

44. Galovski, T.B., Blanchard, E.B., 2002. Psychological characteristics of aggressive drivers with and without intermittent explosive disorder. Behav Res Ther. 40, 11571168.

45. Galovski, T.E., Blanchard, E.B., 2004. Road rage: A domain for psychological intervention? Aggress Violent Behav. 9, 105-127.

46. Álvarez, F.J., Fierro, I., Del Río, M.C., 2007. Cannabis and driving: Results from a general population survey. Forensic Sci Int. 170, 111-116.

47. Blows, S., Ivers, R.Q., Connor, J., Ameratunga, S., Woodward, M., Norton, R., 2005. Marijuana use and car crash injury. Addiction. 100, 605-611.

48. Matthews, A., Bruno, R., Johnston, J., Black, E., Degenhardt, L., Dunn, M., 2009. Factors associated with driving under the influence of alcohol and drugs among an Australian sample of regular ecstasy users. Drug Alcohol Depend. 100, 24-31.

49. European Commission. 2001. White Paper-European transport policy for 2010: Time to decide. Luxembourg: Office for Official Publications of the European Communities.

50. European Monitoring Centre for Drugs and Drug Addiction. 2007. EMCDDA Selected Issue 2007: Drugs and Driving. Luxembourg: Office for Official Publications of the European Communities.

51. Walsh, J., Verstraete, A., Huestis, M., Morland, J., 2008. Guidelines for research on drugged driving. Addiction. 103, 1258-1268. 



\section{Capítulo 7}

\section{Road rage among drug dependent patients}

Benavidez, D.C., Marcos-Flores, A.C., Fierro, I., Álvarez, F.J.

Accid Anal Prev, 2013; 50: 848-853 



\begin{abstract}
The consumption of alcohol, cocaine and cannabis is associated with aggressive behaviour, being a victim of injuries from various causes, and suffering traffic accidents. On the other hand, there is a significant association between road rage and traffic accidents, yet this has not been studied in persons suffering a substance dependence disorder. This study analyses the prevalence of road rage in substance dependent patients undergoing treatment. 100 patients randomly selected at an outpatient treatment centre were included in the study. $63 \%$ of the patients had experienced road rage in the year prior to the interview, and $18 \%$ were serious perpetrators. There was a higher frequency among drivers and those who were starting treatment for cocaine and cocaine + heroin. The study shows that road rage is very frequent among patients with disorders due to substance dependence who are undergoing treatment, in particular the most severe form ("serious perpetrators"). Special attention should be addressed to the issue of driving and road rage during the treatment of these patients.
\end{abstract}

\title{
Keywords
}

Illicit drugs; Substance dependence; Road rage 



\section{Introduction}

The consumption of alcohol, cocaine and cannabis is associated with aggressive behavior [1-10]. There is, however, limited information concerning how the consumption of these substances affects the experience of road rage. Although there is no established definition of road rage at a scientific level [11-13], it can be understood as "an attempt to intimidate, threaten, injure, or kill other drivers, passengers, or pedestrians" [13]. There is epidemiological evidence that victims and perpetrators of road rage, as well as serious road-rage perpetrators - those who intentionally damage or attempt to damage another driver's car or injure or attempt to injure the driver or passenger of another vehicle - have a significantly higher risk of becoming involved in traffic accidents[14]; although it has been pointed out in another study [15] that it only occurs when road rage is expressed through dangerous driving or through direct confrontation with other drivers.

Driving under the influence of alcohol $[16,17]$ has been associated with being a victim and/or a perpetrator of road rage, particularly for those individuals with alcohol related problems (measured according to a subscale of the AUDIT test) [18]. As for drugs, driving under the influence of cannabis has been associated with being both victim and aggressor [17]; while the consumption of stimulants, particularly ecstasy, has been linked to the most severe forms of road rage [1619]. Cocaine has been linked, in particular, to being both victim and aggressor at the same time [16].

Nevertheless, these studies were carried out with the general population, without discriminating between whether the consumption of substances had been recreational or, on the other hand, whether there had been some problem of dependency. In a study carried out among 431 patients undergoing treatment for alcoholism [20], no connection was found between alcoholism and road rage, except among those who had driven while being drunk.

Drivers and non-drivers could be involved in road rage, and both were included in the study. While the role of drivers in road rage is easy to understand, it is harder to interpret the effects of passengers on traffic safety, but as noted by [21], the presence or absence of passengers in the vehicle may influence the 
driver's degree of anger and forms of expression when driving. For example, the presence of older passengers has been associated with lower speeds, whereas young male passengers can have a "speeding-up" effect on young male drivers [22]. Both aspects, a propensity to drive faster and a tendency to drive more slowly, especially in fast-moving traffic, were signalled as possible causes of road rage, increasing the risk of collision involvement [14]. On the other hand, road rage usually appears as an interactive process between victim and aggressor [14], including an exchange of verbal insults which may make the distinction between victim and aggressor more difficult, besides possibly involving both the driver of the vehicle and the passengers.

It seems reasonable to expect an increase in road rage among patients undergoing treatment for drug dependency (anxiety, hostility, etc.) with respect to the general population due to their special psychological characteristics and psychiatric co-morbidity [23], in addition to the possibility that, in some cases, these patients may continue to consume alcohol/drugs or, in other cases, they may be subject to withdrawal symptoms. Although an association between road rage and drug dependence disorder may well be expected, that is to say, expecting greater aggressiveness on the road among those patients who are drug-dependent; to date, this question has not been investigated, which is why the present study has been carried out. The aim of this paper is to analyse the prevalence of road rage among patients undergoing treatment for drug dependence and to establish a comparison with the general population.

\section{Materials and methods}

\subsection{Participants and procedures}

In the current study two datasets have been used: (i) The newly generated data base for patients on drug dependence treatment, and (ii) data from the AlcoholUse and Drug-Use Survey of Castile and Leon, Spain. 


\subsubsection{Drug dependent patients}

The number of patients required was determined according to data obtained from previous studies about road rage in the general population of Castile and Leon [17]. In this study, road rage was observed in $31.1 \%$ [95\% CI $=29.3-32.9$ ]. Initially, we hypothesized a $20 \%$ increment in the prevalence of road rage in drug dependent patients undergoing treatment as compared to the general population. A sample of 100 patients would be needed to detect these increases, so a randomized sample of 100 patients was finally interviewed. Notice that that the sample size "calculation" is for estimating "prevalence" for the whole sample only.

Non-drivers were included in the study as they could be involved in road-rage as victims and/or perpetrators (i.e., a passenger in a car involving road-rage behaviour). Road rage is frequent in the general population of Castile and Leon at $31.1 \%$ (drivers $44.4 \%$ and non-drivers $8.0 \%$, either as victims and/or perpetrators) and is also associated with certain behaviour patterns related with the consumption of alcohol and drugs [17].

The study was approved by the Clinical Research Ethics Committee of the University of Valladolid (CEIC code: Protocol 2011/7).

The study was carried out at the Valladolid Care Centre for Drug Dependents of the Spanish Red Cross, a medical centre accredited by the health authorities. The anonymity of the interviewees was guaranteed; the patients participated voluntarily; they were all aged over 18 and were undergoing outpatient treatment for drug dependence. The patients had been diagnosed by the team of physicians according to the criteria of the DSM-IV TR [24]. The interviews were carried out between 01/02/2011 and 31/07/2011.

The patients were selected at random from among those who attended a weekly urine control (all the patients undergoing treatment in the centre have to have a weekly urine control to evaluate whether they are following the treatment correctly and to verify abstinence) and were in the first 12 months of treatment. 
Information was gathered concerning the participants' sociodemographic factors: gender, age, driving licence, Kilometres driven annually, work activity, sports practised and potentially dangerous tasks carried out in the home. Clinical aspects of the patients enrolled in the study, such as the primary substance for which substance dependence treatment was started, and other drugs consumed, length of treatment, etc., were also recorded. It should be noted that the centre where patients were enrolled was mainly a centre for substance dependence, the inclusion of alcohol dependent patients who usually go to a specific alcohol clinic being infrequent.

\subsubsection{Data from the Alcohol-Use and Drug-Use Survey of Castile and Leon, Spain 2008 [25]}

The database of Alcohol-Use and Drug-Use Survey of Castile and Leon (Spain 2008) [25]; has been used to contrast our hypothesis about sample size and compare results on road rage $[17,26]$. This database was used to obtain prevalence data about road rage and serious perpetrations among the total sample and among those who had taken any drug in the previous year. These later prevalence data had not been analyzed in our previous studies.

Data was collected using the Alcohol-Use and Drug-Use Survey of Castile and Leon, Spain 2008 and through face to-face interviews from April 21-May 22, 2008 [25]. The survey, which has been conducted regularly since the late 1980s, focuses on patterns of alcohol, tobacco, and illicit drug consumption in the general population. In this survey, the same questions regarding road rage as in the current study were addressed. The participants were selected at random from a representative sample of Castile and Leon households that represent 2528417 inhabitants, consisting of 1251082 males and 1277335 females. The sample was taken from the population register data of 2007. People from 14 to 70 years of age living in Castile and Leon, Spain, were the target population. A final sample of 2500 valid interviews was selected, $51 \%(n=1276)$ were males and $49 \%(n=1224)$ females. $63.6 \%(n=1591)$ drove vehicles and $36.4 \%$ $(n=909)$ did not drive. 


\subsection{Road rage questionnaire}

There are various questionnaires designed to measure road rage. Among those most commonly used are: the Propensity for Angry Driving Scale - PADS [27], which identifies drivers more likely to participate in acts of road rage; the Driving Anger Scale - DAS [28], which measures the propensity to experience rage while driving; the Driving Anger Expression Inventory - DAX [29], which measures the way of expressing anger while driving; and finally, the indicators from a taxonomy of road-rage behaviour developed by $[13,30]$, which quantify how frequently someone has been involved in road rage as a victim or a perpetrator over the previous 12 months. Our study used the latter for its ease of administration, having only eight items in the questionnaire; while also allowing the results obtained to be compared with the majority of previous studies on road rage and substance use $[16,18,19]$, having been the most widely used instrument for measuring this phenomenon.

Road rage experience and behaviour for the previous 12 months was measured using eight questions: four items on road rage victimization and four items on road rage perpetration $[13,16,18]$. The victimization items measured how many times, during the previous 12 months, "someone in another vehicle (a) shouted, cursed, or made rude gestures at you or others with you; (b) threatened to hurt you or others with you or threatened to damage the vehicle you were in; (c) intentionally damaged or attempted to damage the vehicle you were in; or (d) intentionally hurt or attempted to hurt you or others with you". The perpetration items measured how many times, during the previous 12 months, the respondent had "(a) shouted, cursed or made rude gestures at a driver or passenger in another vehicle; (b) threatened to hurt a driver or passenger in another vehicle or threatened to damage their vehicle; (c) intentionally damaged or attempted to damage another driver's vehicle; or (d) intentionally hurt or attempted to hurt a driver or passenger in another vehicle".

Taking these items into account, we calculated the percentage of patients that had had some experience of road rage in the previous 12 months, whether as a victim, an aggressor or both. The patients in this group were then divided into subgroups: (a) Only victims, if they had responded affirmatively to at least one 
of the questions in this series, but not to those of the perpetration series; (b) Only perpetrators, in the inverse case and; (c) Victims and perpetrators, if they had responded affirmatively to at least one question in each series. Furthermore, the perpetrators were divided (independently of whether they were also victims or not) [14] into "low" perpetrator or showing "aggressive driving" and "serious" perpetrators. It is considered that a person is a "low" perpetrator or shows "aggressive driving" when he/she responds affirmatively only to the question about whether he/she has shouted, insulted or made gross gestures to a driver or passenger of another vehicle, but not to the rest of the road rage perpetration series; while the connotation of "serious" perpetrator is reserved for those who responded affirmatively to any of the last three items of this series. The database of Alcohol-Use and Drug-Use Survey of Castile and Leon [25] was reanalyzed to obtain prevalence data about road rage and serious perpetrations in consumers of any drug (with or without alcohol) in the previous year.

\subsection{Data analysis}

The program PASW Statistics 18 was used to analyse the information gathered. The results are shown as percentages (and 95\% Confidence Intervals, 95\% CI) or means \pm SD. For categorical variables, group comparisons were conducted employing the Chi-square test. Fisher's exact statistic is reported when, in $2 \times 2$ cross-tables, cell counts fell below expected values. For continuous variables (age and km driven), group comparisons were conducted employing the independent samples Mann-Whitney $U$-test. Statistical significance was defined by $p \leq 0.05$.

\section{Results}

Table 1 shows the sociodemographic characteristics, the substance consumption patterns, treatment and driving patterns of the patients who participated in the study. 100 patients were included in the study, mainly cocaine and heroin dependents $(n=41)$ and heroin only dependents $(n=39)$. 10 patients began the treatment for cocaine only dependence and another 10 were dependent on 
other drugs (cannabis, alcohol, medicaments, amphetamines, etc.). The majority were males $(85 \%)$. The mean age $( \pm S D)$ being $38.2 \pm 8.4$ years, which was similar for males and females (Mann-Whitney $U$-test $=709.0, p>0.05$ ). A great percentage had middle school studies (complete or incomplete), and $71 \%$ were not working. As for the treatment itself, over $2 / 3$ were on the Methadone Maintenance Programme. $45 \%$ of the sample was drivers, more frequently males $(p<0.05)$ and workers $(p<0.001)$. The mean $\mathrm{km}$ driven annually was $22922 \pm 38966 \mathrm{~km}$ (for gender, Mann-Whitney $U$-test $=47.5, p>0.05$ ).

$63 \%$ of the patients had had some experience of road rage in the previous 12 months (Table 2), either as a victim, a perpetrator or both. Of these, $11 \%$ were only victims, $4 \%$ only perpetrators and $48 \%$ both victims and perpetrators. $52 \%$ were perpetrators, 34\% of whom were "low" perpetrators and 18\% "serious" perpetrators. No significant differences were found with respect to age, educational level or work situation. Serious road rage occurred more frequently among males $(21.2 \%)$ than among females $(0 \% ; p<0.05)$.

Drivers $(91.1 \%)$ experienced some form of road rage in the previous year with a greater frequency than non-drivers (40\%; Table 2); while the percentage of drivers who were both victims and perpetrators at the same time was triple that of the non-drivers (75.6\% vs. $25.4 \%$ ). The "low" perpetrators among the drivers were practically double $(46.7 \%)$ the percentage among the non-drivers $(23.6 \%)$, and the "serious" perpetrators more than four times the percentage $(31.1 \%$ vs.7.3\%).

Road rage was higher among drug dependent patients than either the general public or those who had taken some kind of drug in the previous year (Figure 1). This applies to both drivers and non-drivers. As for serious road rage, the trend was similar: $18 \%$ among drug dependent patients, $10.1 \%$ among those who had taken drugs in the previous year and $2.6 \%$ among the general public (Figure 1 ). This also applies to drivers and, to some extent, to non-drivers. 


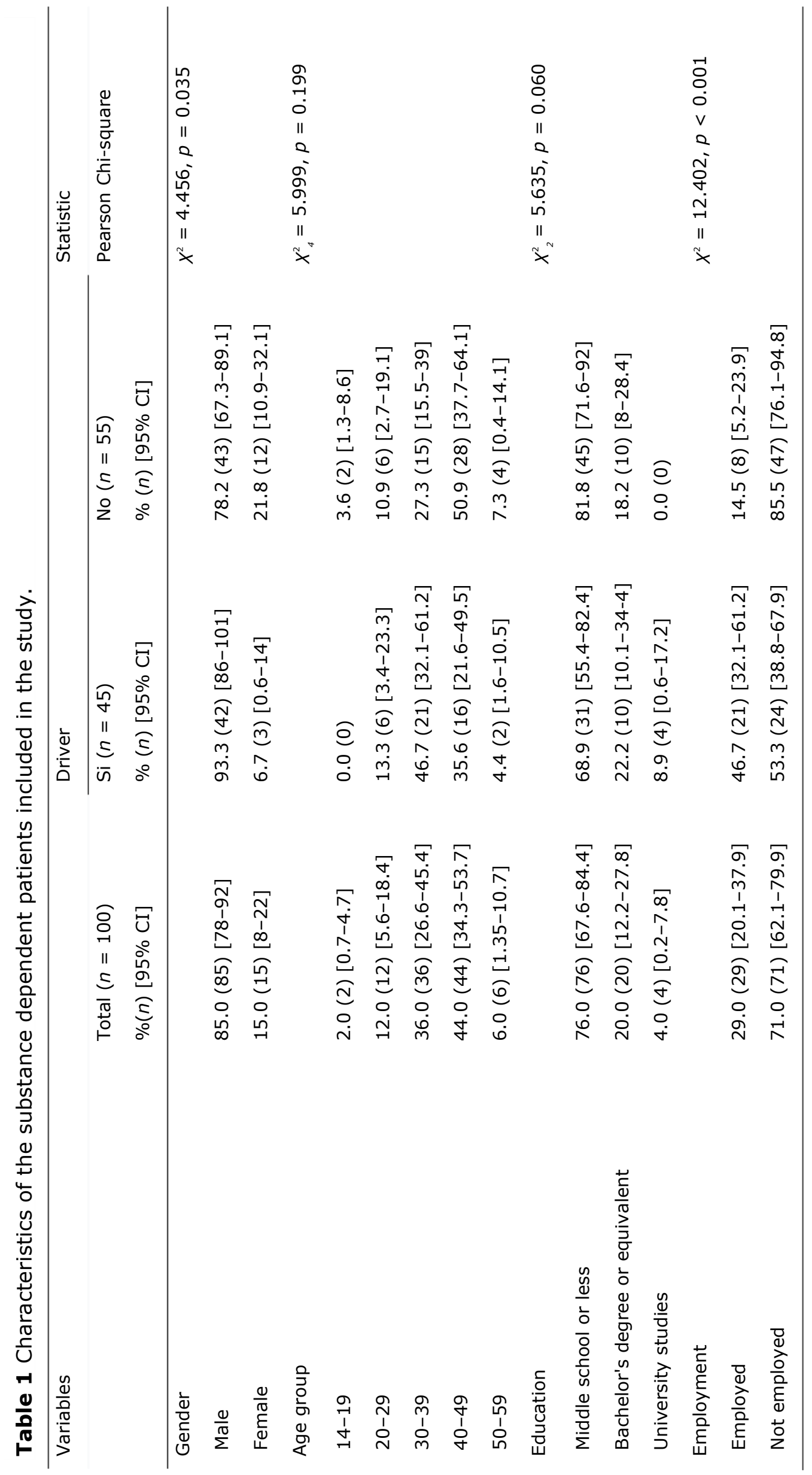




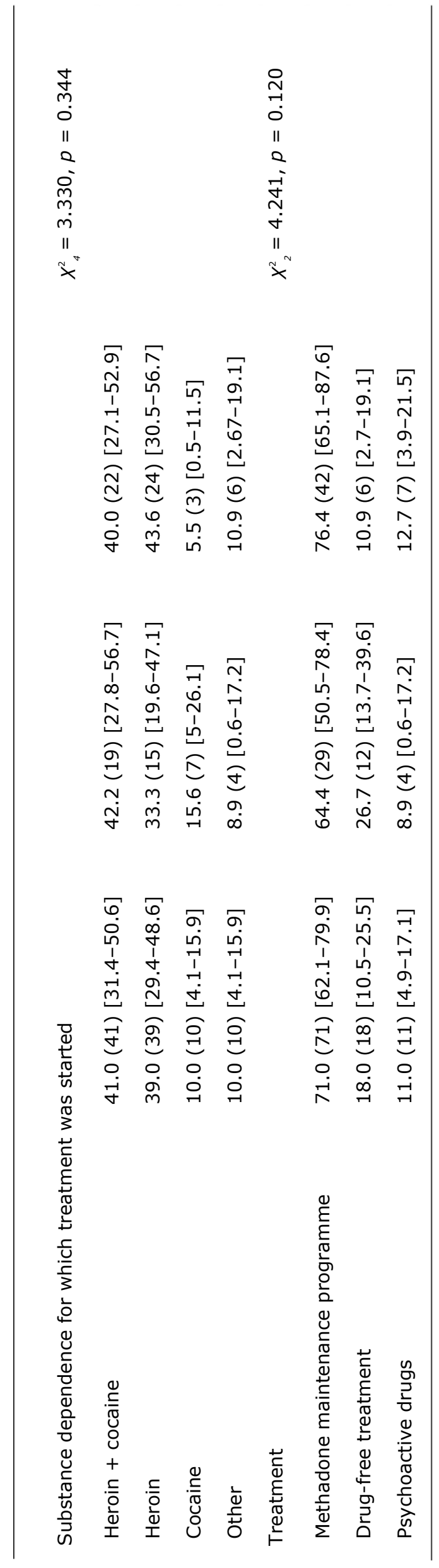




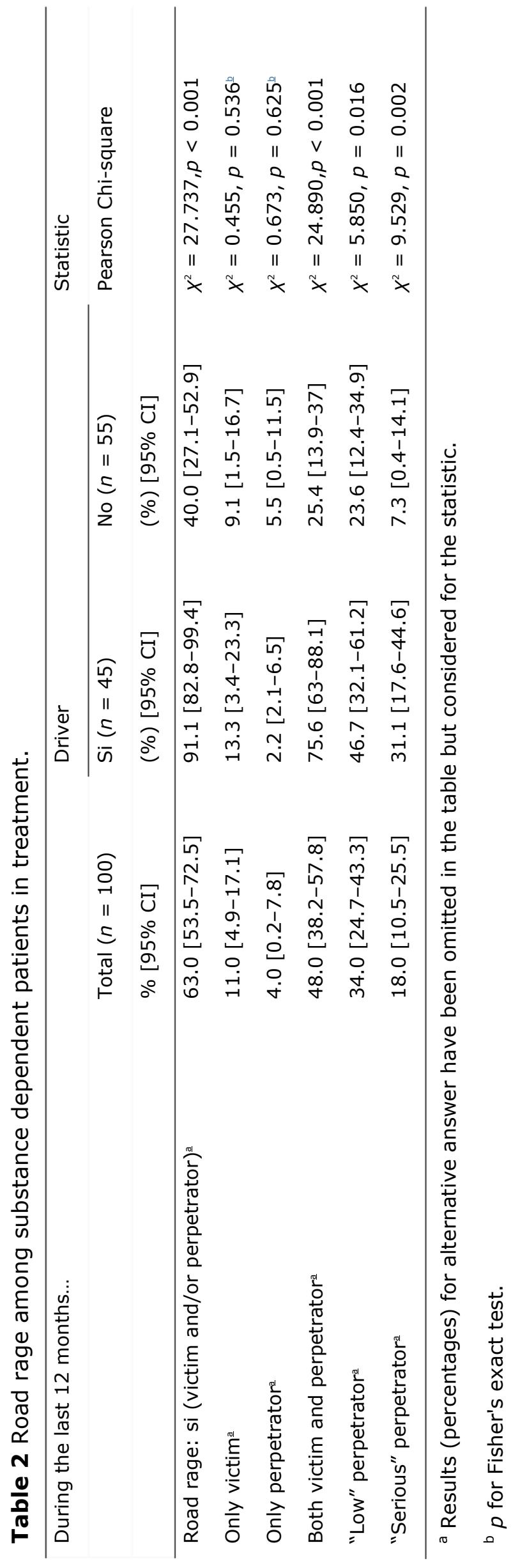




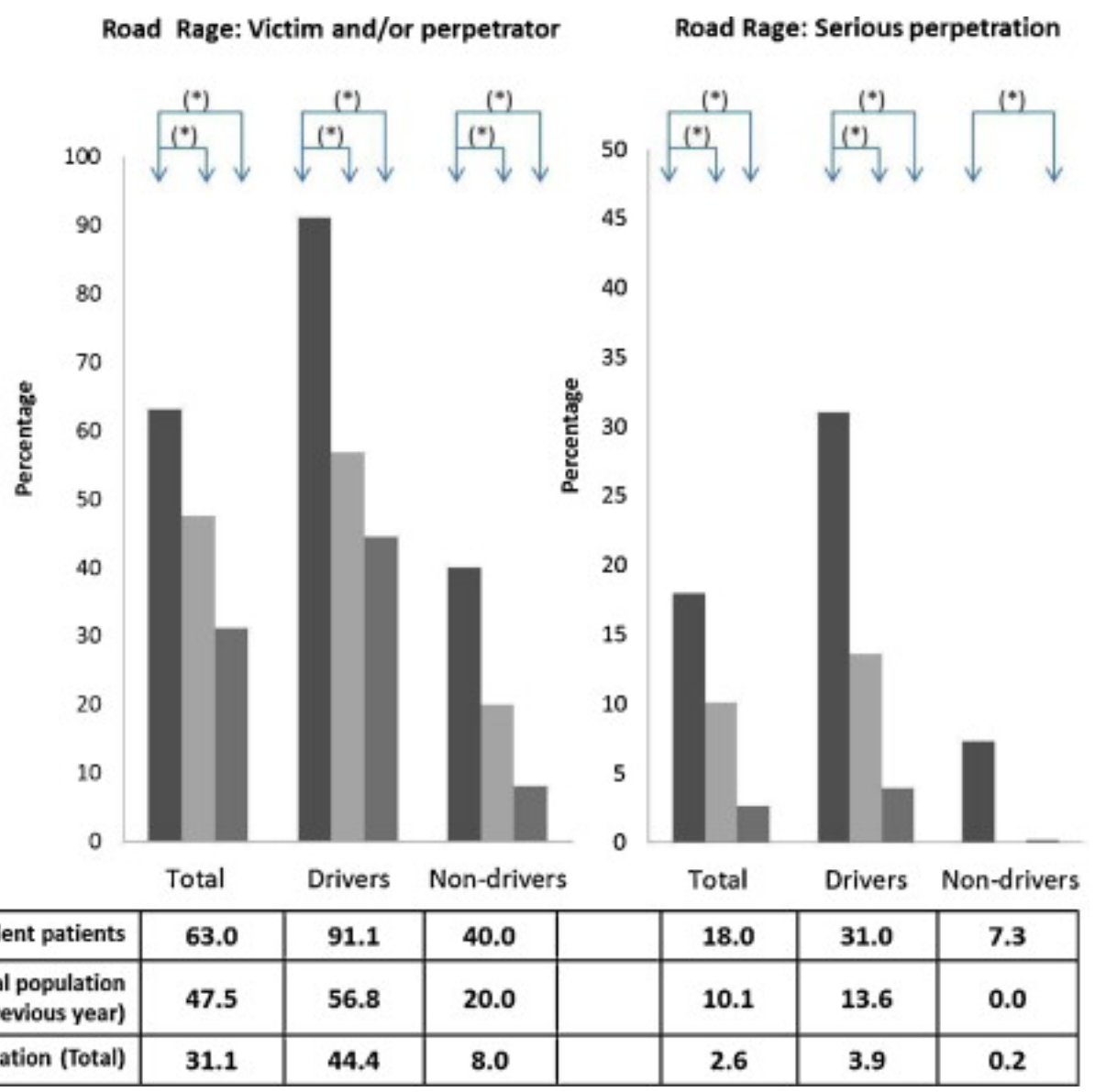

Figure 1 Prevalence of road rage and serious perpetration in dependent patients under treatment, and in the general population, and those who refer to drug use in the previous year. Results for alternative answers ("no road rage" and "no serious perpetration"), have not been presented in the figure but considered for the statistic. Statistical significances $(p \leq 0.05)$ of Chi-square tests marked as $(*)$.

9 out of 10 cocaine dependent patients referred to having experienced road rage in the previous 12 months (Table 3 ). This percentage was $70.7 \%$ among those who were dependent on cocaine + heroin, $51.3 \%$ among those dependent on heroin and $50.0 \%$ among those dependent on other drugs. The high percentage of serious perpetrators among those who were undergoing treatment for cocaine ( 4 out of 10 ) and those undergoing treatment for cocaine + heroin (22\%) must be noted. 


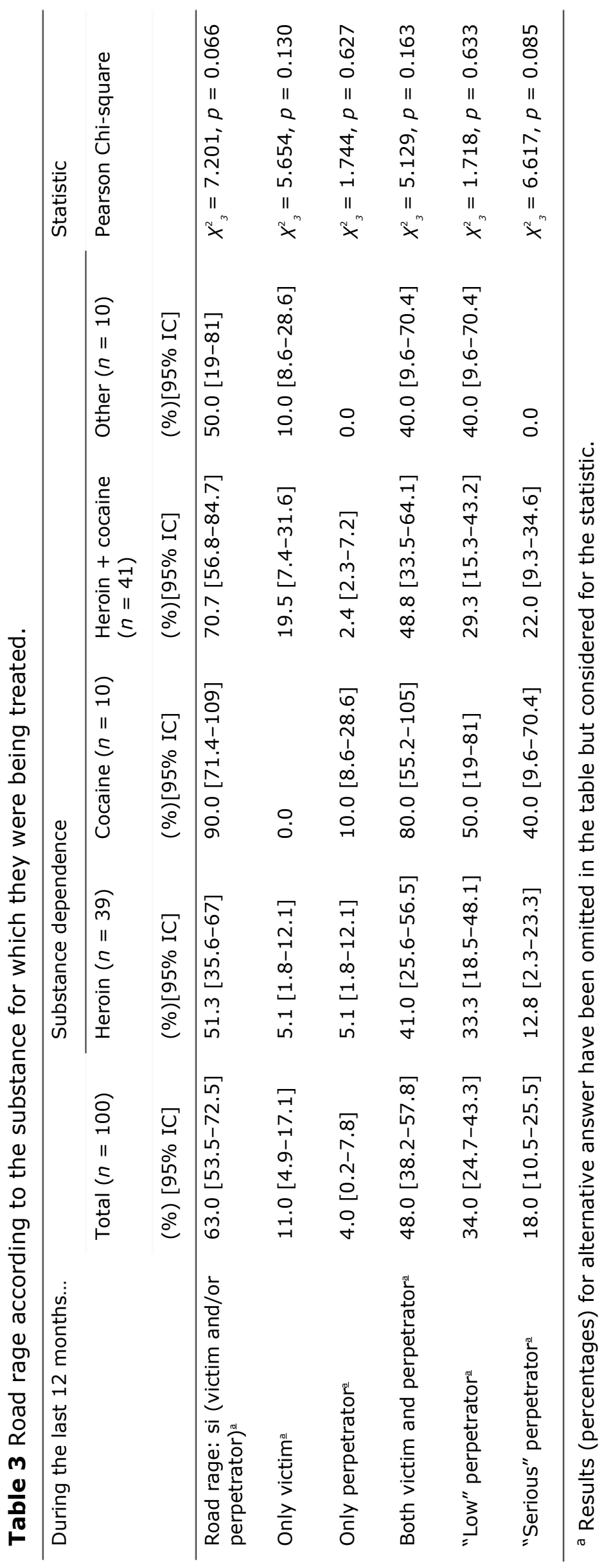




\section{Discussion}

This study shows that nearly two out of three patients in treatment for their drug dependency have reported road rage in the previous year. This frequency is higher than those reported for the general population, even when comparing with consumers of drugs in the previous year. The percentage of "serious" perpetrators was worth noting. In studies carried out in Canada in 2001 and $2003,47.5 \%$ and $40.6 \%$ of the population, respectively, admitted having had experience of road rage during the previous year, $31.0 \%$ and $33.2 \%$ being perpetrators for each year [13].

Fierro et al. carried out a study of the general population of Castile and Leon, in which $31.1 \%$ of those interviewed admitted having had experience of road rage over the previous 12 months [26]. In this current study, carried out with drug dependent patients from the same geographical region, the percentage of patients who had experienced road rage (63\%) was double the previous one. Perpetrators reached 52\%; of whom the "low" perpetrators (34\%) were approximately double those from the study of the general population $(17.6 \%)$; while the percentage of "serious" perpetrators (18\%) was almost 7 times greater than the other (2.6\%). In other studies of the general population of Canada [31], the percentage of low perpetrators (31.7\%) was not that different from that of our patients $(34 \%)$, but in the case of the serious perpetrators, the difference was similar to that already mentioned ( $2.1 \%$ as opposed to $18 \%$ in our patients). We find a high prevalence of habitual drivers among patients undergoing treatment for substance dependence (45\%). As expected, road rage was closely related to the fact of being a driver. Those who did not drive any vehicle can have experienced it, but to a much lesser degree. Clearly, it is drivers who are much more likely to be involved in road rage situations, and thus have the higher percentages. Very significant differences were also observed among the general population between drivers and non-drivers for road rage in its many different forms $[17,26]$. Even so, the percentages found in our patients were, in all cases, much higher than those of the general population, for both drivers and nondrivers. 
When reanalysing the data for the general population, and considering those who reported drug use within the previous year, we have observed a progression in road rage/serious perpetrators in relation with substance use: figures are higher for those who are dependent than for those who refer to some kind of drug use in the previous year, and finally the general population.

Although there are factors, such as that of being a driver, which are closely related with road rage, it is also clear that there is a relationship between substance use and road rage. Fierro et al. found that a driver under the influence of cannabis, or alcohol and cannabis, was associated with being a victim and a perpetrator of road rage [17]. Butters et al. found an association between the consumption of cannabis in the previous 12 months and experience as a victim and a perpetrator, but not as a "frequent perpetrator" $[16,19]$. As for cocaine, its consumption in the previous 12 months was associated with experiencing road rage as both a victim and a perpetrator [16].

Road rage, and especially "serious" road rage, was frequently found among cocaine and cocaine + heroin dependent patients. The study was not designed to assess differences regarding substance use type, and therefore, this relevant finding should be confirmed in later studies. In any case, before drawing conclusions concerning the consumption of substance type and its relationship to road rage, it is necessary to consider the matter carefully and to remember that patients undergoing treatment have relapses and sporadic periods of consumption in which they can consume the substance for which treatment started, or other substances.

The association between the consumption of illicit drugs and road rage could be explained by a direct pharmacological effect. There is important evidence in the literature relating stimulants with aggressive behavior [5,8,9,32]. In some cases, road rage could be possible due to the fact that the patients were experiencing withdrawal symptoms. Nevertheless, there are alternative explanations for this association. It is possible that those individuals who report frequent aggressive behaviour on the roads share factors of a social nature or personality traits that also link them to the consumption of drugs [18]. In other words, the use of these substances could, in some way, lead to road rage; or both behaviour patterns 
could share a common origin, as suggested by the Theory of Problematic Behaviour [33], according to which there would be an underlying causal mechanism that could explain a wide range of activities that involve taking risks. Serious perpetrators of road rage could be individuals immersed in an aberrant lifestyle, which may include relatively frequent law-breaking and a general indifference to legal sanctions [33]. For these individuals, road rage would simply be a manifestation of their general propensity towards anti-social and criminal conduct.

The present study has some limitations which should be pointed out. Firstly, the study has included a limited number of patients to test whether drug dependent patients have higher figures for road rage than the general population; so the findings should be confirmed in later studies. On the other hand, it is based on self-reported data concerning road rage, rather than information from an objective source, such as traffic camera video recordings. Thus, the patients' answers cannot be verified. In addition, self-reporting can lead to overestimations or omissions, especially when the information being asked for is some time in the past (some of the questions refer to facts that could have taken place up to 12 months prior to the interview). However, others have reported good validity for self-evaluation information among drug users [34]. Furthermore, over or under reported road rage could be biased towards social desirability. Other limitations are that, due to the descriptive design of the study, causal relationships cannot be inferred, while the test on road rage, although used in previous studies, was not validated in the population in which the study was carried out.

The relationship between the consumption, abuse of and/or dependence on substances and road rage undoubtedly needs to be explored more extensively. The understanding of the underlying mechanisms through which these behaviour patterns are associated with each other is of great importance and will allow us to tailor legislation and treatment to the patients' real situation. Furthermore, patients included in the study were under treatment. We have no information about the occurrence of road rage in substance dependents without treatment, and whether simply entering treatment has any effect, or not, on road rage. 


\section{Conclusion}

The present study shows that road rage is very frequent among patients undergoing treatment for substance dependence, in particular the most severe form ("serious perpetrators"), and that, in spite of the treatment being carried out, the percentages of the different forms of road rage are far higher than those for the general population. Considering that road rage has been related with a greater incidence of traffic accidents [14], these patients make up an important target group for the prevention of road traffic accidents. Health professionals and policy makers should be aware of this, and implement appropriate measures to reduce and avoid this type of behaviour. The involvement of health service personnel from the treatment centres is essential, not only to give information about the risks of such behaviour, but especially in designing the treatment programmes, since the effectiveness of such psychological intervention programmes in drivers committing acts of road rage has already been demonstrated [35]. The possibilities for developing road rage preventive measures have been reviewed recently [36], and actions at legal, judicial (courtbased programmes), car redesign, mass-media campaigns, and changes in society, have all been proposed.

\section{Conflict of interest}

The authors declare that no conflict of interest exists.

\section{Acknowledgements}

This study has been supported by a grant from the "Instituto de Salud Carlos III"/FEDER for the development of the Thematic Networks of Cooperative Research, Addictive Disorders Network RD06/0001/0020 and the support of the European Commission through the EADIC lot 16 - Erasmus Mundus programme. We would also like to express our gratitude to the Drug Dependence Treatment Centre of the Red Cross in Valladolid. 


\section{References}

1. Grisso, J.A., Schwarz, D.F., Hirschinger, N., Sammel, M., Brensinger, C., Santanna, J., Lowe, R.A., Anderson, E., Shaw, L.M., Bethel, C.A., Teeple, L., 1999. Violent injuries among women in an urban area. N Engl J Med. 341, 1899-1905, http://dx.doi.org/10.1056/NEJM199912163412506.

2. Macdonald, S., Anglin-Bodrug, K., Mann, R.E., Erickson, P., Hathaway, A., Chipman, M., Rylett, M., 2003. Injury risk associated with cannabis and cocaine use. Drug Alcohol Depend. 72, 99-115.

3. Mann, R.E., Anglin, L., Wilkins, K., Vingilis, E.R., MacDonald, S., 1993. Mortality in a sample of convicted drinking drivers. Addiction. 88, 643-647.

4. Mann, R.E., Suurvali, H.M., Smart, R.G., 2001. The relationship between alcohol use and mortality rates from injuries: a comparison of measures. Am J Drug Alcohol Abuse. 27, 737-747.

5. Moss, H.B., Tarter, R.E., 1993. Substance abuse, aggression, and violence. Am J Addict. 2, 149-160, http://dx.doi.org/10.1111/j.1521-0391.1993.tb00215.x.

6. Pernanen, K., 1991. Alcohol in Human Violence. Guilford Press, New York.

7. Potter, C.C., Jenson, J.M., 2003. Cluster profiles of multiple problem youth. Crim Justice Behav. 30, 230-250, http://dx.doi.org/10.1177/0093854802251007.

8. Reiss, A., Roth, J., 1993. Understanding and Preventing Violence. National Academy Press, Washington, DC.

9. Smart, R.G., Mann, R.E., Tyson, L.A., 1997. Drugs and violence among Ontario students. J Psychoactive Drugs. 29, 369-373.

10. Wells, S., Graham, K., West, P., 2000. Alcohol-related aggression in the general population. J Stud Alcohol. 61, 626-632.

11. Smart, R.G., Mann, R.E., 2002. Is road rage a serious traffic problem? Traffic Inj Prev. 3, 183-189.

12. Smart, R.G., Mann, R.E., 2002. Deaths and injuries from road rage: cases in Canadian newspapers. CMAJ. 167, 761-762.

13. Smart, R.G., Mann, R.E., Zhao, J., Stoduto, G., 2005. Is road rage increasing? Results of a repeated survey. J Safety Res. 36, 195-201.

http://dx.doi.org/10.1016/j.jsr.2005.03.005. 
14. Mann, R.E., Zhao, J., Stoduto, G., Adlaf, E.M., Smart, R.G., Donovan, J.E., 2007. Road rage and collision involvement. Am J Health Behav. 31, 384-391. http://dx.doi.org/10.5555/ajhb.2007.31.4.384.

15. Wells-Parker, E., Ceminsky, J., Hallberg, V., Snow, R.W., Dunaway, G., Guiling, S., Williams, M., Anderson, B., 2002. An exploratory study of the relationship between road rage and crash experience in a representative sample of US drivers. Accid Anal Prev. 34, 271-278.

16. Butters, J.E., Smart, R.G., Mann, R.E., Asbridge, M., 2005. Illicit drug use, alcohol use and problem drinking among infrequent and frequent road ragers. Drug Alcohol Depend. 80, 169-175. http://dx.doi.org/10.1016/j.drugalcdep.2005.03.018.

17. Fierro, I., Morales, C., Alvarez, F.J., 2011. Alcohol use, illicit drug use, and road rage. J Stud Alcohol Drugs. 72, 185-193.

18. Mann, R.E., Smart, R.G., Stoduto, G., Adlaf, E.M., Ialomiteanu, A., 2004. Alcohol consumption and problems among road rage victims and perpetrators. J Stud Alcohol. 65, 161-168.

19. Butters, J.E., Mann, R.E., Smart, R.G., 2006. Assessing road rage victimization and perpetration in the Ontario adult population: the impact of illicit drug use and psychiatric distress. Can J Public Health. 97, 96-99.

20. Yu, J., Chin Evans, P., Perfetti, L., 2004. Road aggression among drinking drivers: alcohol and non-alcohol effects on aggressive driving and road rage. J Crim Justice. 32, 421-430. http://dx.doi.org/10.1016/j.jcrimjus.2004.06.004.

21. Shinar, D., Compton, R., 2004. Aggressive driving: an observational study of driver, vehicle, and situational variables. Accid Anal Prev. 36, 429-437.

22. Baxter, J.S., Manstead, A.S.R., Stradling, S.G., Campbell, K.A., Reason, J.T., Parker, D., 1990. Social facilitation and driver behavior. Br J Psychol. 81, 351-360.

23. Hemenway, D., Vriniotis, M., Miller, M., 2006. Is an armed society a polite society? Guns and road rage. Accid Anal Prev. 38, 687-6955.

24. American Psychiatric Association, 2000. DSM-IV-TR: Diagnostic and Statistical Manual of Mental Disorders, 4th text revision ed. American Psychiatric Association, Washington, DC.

25. Álvarez, F.J., Fierro, I., 2010. Las drogas en Castilla y León, 2008. Consejería de Familia e Igualdad de Oportunidades, Junta de Castilla y León, Valladolid.

26. Fierro, I., Gómez-Talegón, T., Álvarez, F.J., 2010. Agresividad vial en la población general. Gac Sanit. 24, 423-427. http://dx.doi.org/10.1016/j.gaceta.2010.07.004. 
27. De Pasquale, J.P., Geller, E.S., Clarke, S.W., Littleton, L.C., 2001. Measuring road rage: development of the propensity for angry driving scale. J Safety Res. 32, 1-16.

28. Deffenbacher, J.L., Oetting, E.R., Liynch, R.S., 1994. Development of a driving anger scale. Psychological Reports. 74, 83-91.

29. Deffenbacher, J.L., Liynch, R.S., Oetting, E.R., Swain, R.C., 2002. The driving anger expression inventory: a measure of how people express their anger on the road. Behav Res Ther. 40, 717-737.

30. Smart, R.G., Stoduto, G., Mann, R.E., Adlaf, E.M., 2004. Road rage experience and behaviour: vehicle, exposure and driver factors. Traffic Inj Prev. 5, 343-348.

31. Smart, R.G., Mann, R.E., Stoduto, G., 2003. The prevalence of road rage: estimates from Ontario. Can J Public Health. 94, 247- 250.

32. Cherek, D.R., Steinberg, J.L., Kelly, T.H., Robinson, D., 1987. Effects of damphetamine on aggressive responding of normal male subjects. Psychiatry Res. 21, 257-265.

33. Donovan, J.E., Jessor, R., 1985. Structure of problem behavior in adolescence and young adulthood. J Consult Clin Psychol. 53, 890-904.

34. Harrison, L., Hughes, A., 1997. Introduction-the validity of self-reported drug use: improving the accuracy of survey estimates. NIDA Research Monograph. 167, 1-16.

35. Galovski, T.E., Blanchard, E., 2004. Road rage: a domain for psychological intervention? Aggress Violent Behav. 9, 105-127.

36. Asbridge, M., Smart, R.G., Mann, R.E., 2006. Can we prevent road rage. Trauma Violence Abuse. 7, 109-121.

Corresponding author at: Pharmacology and Therapeutics, Faculty of Medicine, University of Valladolid, Ethics Review Board, Hospital Clínico Universitario, C/Ramón y Cajal 7, 47005 Valladolid, Spain. Tel.: +34 983 423077; fax: +34 983 423022. 

Capítulo 8

Driving under the influence of drugs in Spain, 2013: decrease prevalence after introduction of onsite drug test 



\section{Abstract}

Background: Studies have shown that the use of drugs increases the risk of traffic accidents. On the other hand, adequate law enforcement policies and the continuity of roadside drug tests have been considered important deterrent factors for driving under the influence of substances (DUI). The aim of this study was to compare the evolution of the presence of alcohol and drugs among Spanish drivers between 2008 and 2013.

Methods: This study was designed as a comparison of two separate representative samples from the general population of Spanish drivers $(n=2932$ drivers in 2013 and $n=3302$ in 2008-2009).

Results: Between 2008 and 2013 a decrease in the prevalence of alcohol (from $4.92 \%$ [4.18-5.66] to 3.41\% [2.27-4.07], p<0.05) and drug positive cases was observed (from 6.93\% [6.07-7.80] to 4.87\% [4.09-5.65], $p<0.01$ ). The decreased prevalence of drugs was due to a marked drop in the prevalence of THC in drivers, although there were no differences observed in each of the other categories of illegal substances.

Conclusion: The difference in the prevalence of drugs may be related to the increase in roadside drug testing but the deterrent effects of random drug testing depends on multiple factors. Thus, the deterrence effect must be subsequently evaluated.

\section{Keywords}

Substance abuse detection; Driving while intoxicated; Saliva; Breath tests; drug utilization. 



\section{Introduction}

Driving under the influence of substances other than alcohol has been subject of increasing interest over the last decades [1]. As with alcohol, studies have shown that drug intake increases the risk of traffic accidents [2]. Intervention in this area is recognized as a worldwide priority. Persuading drivers that there is a real risk of their drug use being detected is a key deterrent factor for DUI $[3,4]$. Taking this into account, adequate law enforcement policy and the continuity of roadside testing for drug use in drivers play an important role $[5,6]$.

For the European Project DRUID (http://www.druid-project.eu), roadside surveys were conducted in 13 countries across Europe, and results showed a large difference in the prevalence of alcohol/drug intake from country to country [1]. The highest prevalence of alcohol/drug use by drivers was found in Southern Europe, Italy, Spain, and Portugal. In Spain, avoiding driving after alcohol or drug use has been recognized as a top priority to improve road safety. Five years after the DRUID project in Spain, a new roadside survey was conducted with a similar methodology in order to evaluate the evolution of the presence of alcohol and drug use in Spanish drivers.

\section{Methods}

Target population: Motor vehicle drivers, excluding bikers and drivers of vehicles over $3500 \mathrm{~kg}$, on Spanish public roads.

Design: The prevalence of drivers under the influence of substances was monitored over time, and we compared the results of two cross-sectional studies conducted in 2008 and 2013 in separate representative samples of the general population of Spanish drivers. Drivers were selected at random from the total population of drivers using a sampling scheme stratified by country areas, time period, population size, and road type, following the DRUID criteria as previously described Gómez-Talegón et al., [7]. A total of 128 police roadside checkpoints were selected. The data were weighted according to the traffic intensity at each of the 128 points selected. The study included a total simple size of 6234 drivers: 3302 in 2008-2009 (with samples taken between September 26th, 2008 and 
August 24th, 2009) and 2932 in 2013 (with samples taken at two different time periods, May and November).

Substances analyzed and toxicological aspects:

Roadside police controls were legally regulated with mandatory alcohol tests and on-road saliva analysis. Breath tests for alcohol were carried out using the Dräger Alcotest® 6810 device and an on-site oral fluid drugs test, with the Dräger Drug Test $® 5000$ analyzer and test kits for multiple and simultaneous detection of amphetamines, methamphetamines, opiates, cocaine, cannabis and benzodiazepines. The same cut-offs were used in both studies. The oral fluid samples used in the confirmatory analysis were collected with the StatSure ${ }^{\mathrm{TM}}$ Saliva•Sampler ${ }^{\mathrm{TM}}$ for the first group of samples (2008-2009) and with Quantisal ${ }^{\mathrm{TM}}$ in the second (2013). The storage process, cooling process, transportation and subsequent analysis in the laboratory has previously been described [7]. In 2013, only the samples of drivers testing positive in the screening were later analyzed in the laboratory. Consequently the variables of the 2008 study were recodified following the same criteria. Samples were categorized as "negative" when the drivers' results came up negative in the screening test. A final positive result was defined as surpassing the cut-off established in the DRUID project for any of the analyzed substances in the laboratory or having a breath alcohol concentration of $>0.05 \mathrm{mg} / \mathrm{l}$.

Variables: Positives in the screening were defined as 4 dichotomous variables (si/no): "only alcohol", "only drugs", "alcohol and drugs" and "any substance". Regarding confirmatory analysis, two variables were considered: "any substance" (si/no) and a categorical variable with nine groups: negative/THC positive /cocaine positive /amphetamine positive/opiate positive/alcohol positive/benzodiazepine positive/positive for a combination of drugs / alcohol + drug positive.

Statistical analysis: Absolute and relative frequencies with $95 \% \mathrm{CI}$ are shown. The two-proportion z-test was used to determine whether the difference between two proportions was significant. Statistical analyses were carried out with the Statistical Package for the Social Sciences (SPSS, v19). The level of significance was set at $p \leq 0.05$. 


\section{Results}

Screening Results: In $201312.07 \%$ of the on-road traffic controls tested positive for alcohol and/or drugs in the screening test, which shows a decrease of $4.27 \%$ with respect to 2008 (16.34\% Table 1). In 2013, less frequency of cases testing positive for alcohol + drugs was also observed. Compared to 2008, there was a decrease in the prevalence of cases testing positive for alcohol (and negative for drugs) as well as cases testing positive for drugs (and negative for alcohol).

Results of Confirmatory Analysis: In 2013, 9.34\% of cases were confirmed to have some substance present. Compared to $14.05 \%$ in 2008 , this indicates a decrease by $4.71 \%$ (Table 1 ). A decrease was observed in the prevalence of cases testing positive for alcohol and drugs from 2008 to 2013. Upon analyzing the different combination possibilities of substances, differences were observed in the cases testing positive only for alcohol, alcohol + drugs, and for drugs without the presence of alcohol or medicines (2008: 6.93\% [6.07-7.80]; 2013: $4.87 \%[4.09-5.65] ; p<0.01)$. The decrease in prevalence of drugs is due to a less frequent presence of cannabis, while the other categories of illegal drugs showed no change in their prevalence (Table 1 ). 


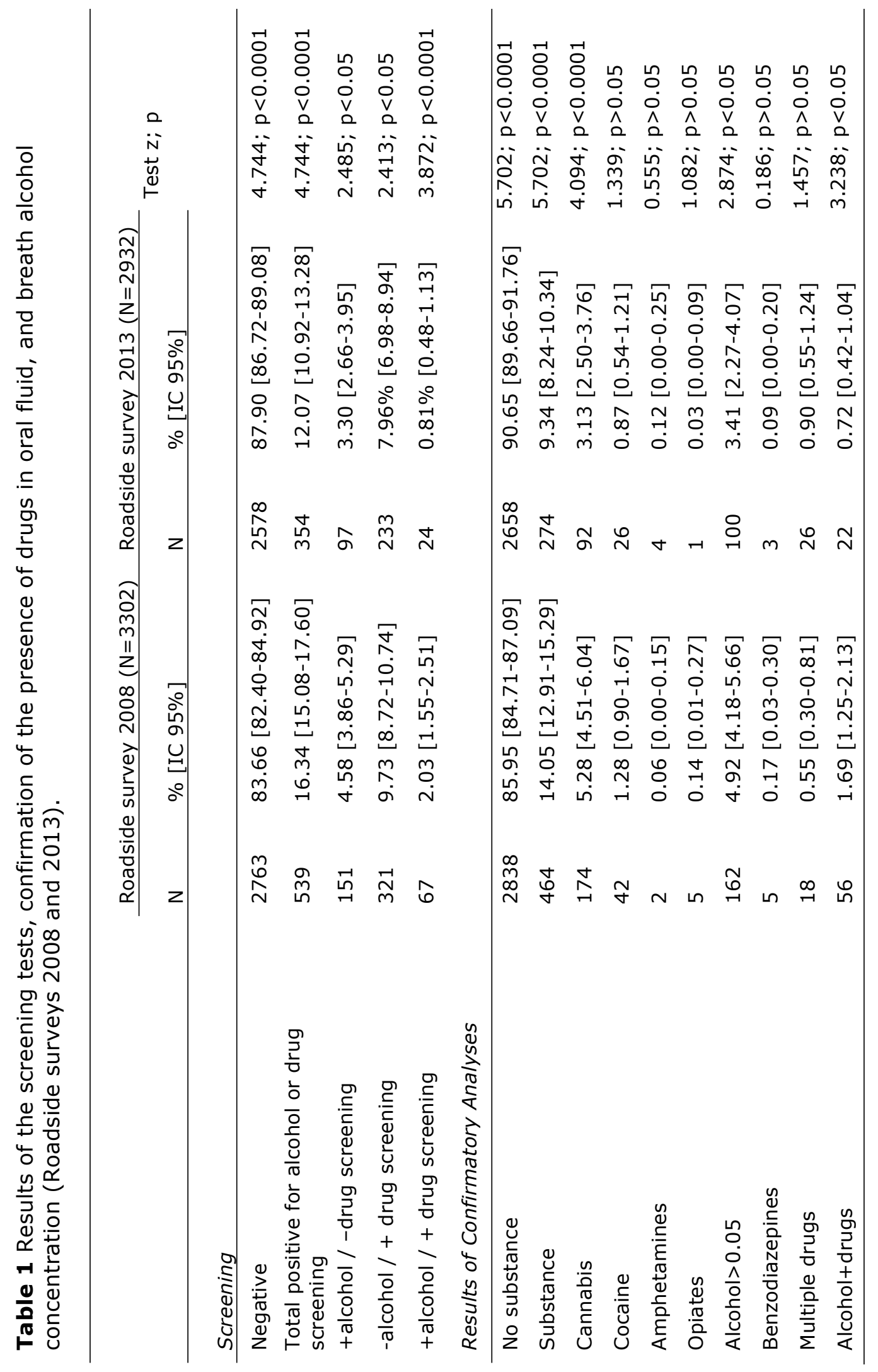




\section{Discussion}

The results show that the presence of alcohol, drugs, and medicines continues to be frequent in Spanish drivers. The data corresponding to 2008 and gathered in the context of the European DRUID project show that Spain (together with Italy), was one of the countries in which the prevalence of drivers who have been exposed to substances was the most frequent, 3.35 times the average frequency observed in the 13 participating countries [1]. The data corresponding to 2013 clearly show a decrease in the prevalence of cases that tested positive for alcohol and/or drugs in the roadside screening test (a decrease of $4.27 \%$ ) as well as in the laboratory test confirmation (a decrease of $4.71 \%$ ). Even when the results are favourable, the prevalence of cases with drivers under the influence of alcohol and drugs is excessively high, and shows the need for intervention in this area.

From a practical point of view, the results of the screening tests are decisive when it comes to police procedure. In some countries, including Spain, when there is a case in which the alcohol screening has tested positive, then there is no further drug testing. One can observe that in the DRUID and present study, the cases testing positive for alcohol are those that show a breath alcohol concentration of $0.05 \mathrm{mg} / \mathrm{L}$ or more. Nevertheless, the legal limit in Spain is 0.25 $\mathrm{mg} / \mathrm{L}(0.15 \mathrm{mg} / \mathrm{L}$ in novel drivers). The 2013 data show a reduction in the cases testing positive in alcohol, and in the cases positive for alcohol plus drugs.

Medicines make up a case in and of itself. Substances analyzed in the DRUID project as well as in the present study include benzodiazepines and particular hypnotic drugs. Certain opiates have also been taken into account. As a consequence, the information obtained from the DRUID project and this study is limited to those specific pharmaceutical groups and only represents part of the problem of drivers under the influence of medicines. Benzodiazepines can be used in the treatment of mental disorders or can be used in an abusive way. From the prospective of safety and traffic security, these are two different problems. In 2008, the presence of pharmaceuticals in 24 drivers was confirmed, with 8 drivers in 2013. On the other hand, Spain has introduced a pictogram of medicines and driving, with the goal of better informing patients of the effects of 
medicines on driving [8]. Benzodiazepines, hypnotic drugs, and opiates show the pictogram on their packages. At the same time, in the context of the European DRUID project, medicines were categorized in four groups [9].

In the current study from 2013, the same criteria has been used a that used in the design of the 2008 study, except that in 2008 the controls were carried out over 11 months, and in 2013 they were carried out in two transversal cuts in May and November. The same criteria were used and the cut-offs as well as the confirmation. Saliva was collected with the StatSure ${ }^{T M}$ in 2008 and Quantisal ${ }^{T M}$ in 2013. A decrease in the prevalence of drugs was observed, even when taking into account that the amount of drug recovered from the Quantisal ${ }^{\mathrm{TM}}$ device was probably higher than the StatSure ${ }^{\mathrm{TM}}$, especially for the THC.

One important question to consider is what factor or factors have caused the apparent decrease in cases testing positive for substances in the present study. Although this study's design does not allow us to for determine all the factors behind this effect, the routine implementation of these tests could have been an important contributing factor to the decrease. As previously pointed out [10], "the experience of random testing in traffic in Victoria (Australia) showed that on-site screening devices have a good performance as a deterrent, leading to a marked decrease in the prevalence of THC, MDMA and methamphetamine, the three drugs which are covered in the states legislation". However, the deterrence effect depends on the frequency of testing as well as the context and may vary significantly with time [6]. Because of this, the deterrence effects of random drug testing and the legislative framework must be subsequently evaluated as well as its usefulness in promoting road safety.

In 2010, a legislative measures were introduced which established the validity of oral fluid (saliva) as biological evidence for testing and made the testing obligatory along with the consequent confirmatory analysis if the screening test were to come up positive [11]. In 2014, new regulation [12] has been introduced which, among other aspects, increases the fine to 1000 Euro (and a loss of 6 penalty points) for driving under the influence of drugs and a zero tolerance for drugs is implemented. 
In conclusion, the present study shows a decrease in the prevalence of cases testing positive for substances in Spanish drivers. Despite the marked decrease, results show that the presence of alcohol, drugs, and medicines continue to be excessively frequent in Spanish drivers. In the area of road safety, preventing driving under the influence of substances is a priority, particularly in our country where new legislation (2014) has been introduced which has, among other aspects, notably increased the fines for driving under the influence. Political dedication to incrementing drug controls on the motorways exists as well. The implementation of these measures in Spain has proven to help make driving under the influence of drugs less and less frequent.

\section{Acknowledgements}

This study has been possible thanks to the collaboration of the traffic police. We also wish to express our thanks to the staff of the laboratory of the Forensic Toxicology Service, of the University of Santiago de Compostela, who carried out the toxicological analysis by LC-MS/MS quantification. Funding for this study was provided by grants from the EU 6th Framework Programme, DRUID project, DGT (No. Exp::0100DGT23059) and the Instituto de Salud Carlos III, Redes Temáticas de Investigación Cooperativa, Red de Trastornos AdictivosRD12/0028/00212.

\section{Conflict of interest}

The author confirms that there are no conflicts of interests. 


\section{References}

1. Schulze, H., Schumacher, M., Urmeew, R., Auerbach, K., Alvarez, F.J., Bernhoft, I.M., de Gier, H., Hagenzieker, M., Houwing, S., Knoche, A., Pilgerstorfer, M., Zlender, B., 2012. Driving Under the Influence of Drugs, Alcohol and Medicines in Europe findings from the DRUID project. European Monitoring Centre for Drugs and Drug Addiction. EMCDDA in Lisbon. Retrieved 2nd October 2014 from http: //www.emcdda.europa.eu/attachements.cfm/att_192773_EN_TDXA12006ENN.pdf

2. Elvik, R., 2013. Risk of road accident associated with the use of drugs: a systematic review and meta-analysis of evidence from epidemiological studies. Accid Anal Prev. 60, 254-67.

3. Chu, M., Gerostamoulos, D., Beyer, J., Rodda, L., Boorman, M., Drummer, O.H., 2012. The incidence of drugs of impairment in oral fluid from random roadside testing. Forensic Sci Int. 215, 28-31.

4. Watling, C.N., Palk, G.R., Freeman, J.E., Davey, J.D., 2010. Applying Stafford and Warr's reconceptualization of deterrence theory to drug driving: can it predict those likely to offend? Accid Anal Prev. 42, 452-8.

5. Shepherd, J.P., 2001. Criminal deterrence as a public health strategy. Lancet, 358, 1717-22.

6. Watson, B., Freeman, J., 2007. Perceptions and experiences of random breath testing in Queensland and the self-reported deterrent impact on drunk driving. Traffic Inj Prev. 8, 11-19.

7. Gómez-Talegón, T., Fierro, I., González-Luque, J.C., Colás, M., López-Rivadulla, M., Álvarez, F.J., 2012. Prevalence of psychoactive substances, alcohol, illicit drugs, and medicines, in Spanish drivers: a roadside study. Forensic Sci Int, 223, 106-113.

8. Fierro, I., Gómez-Talegón, T., Alvarez, F.J., 2013. The Spanish pictogram on medicines and driving: The population's comprehension of and attitudes towards its use on medication packaging. Accid Anal Prev. 50, 1056-61.

9. Ravera, S., Monteiro, S.P., de Gier, J.J., van der Linden, T., Gómez-Talegón, T., Alvarez, FJ., DRUID Project WP4 Partners., 2012. A European approach to categorizing medicines for fitness to drive: outcomes of the DRUID project. Br J Clin Pharmacol. 74, 920-31.

10. DRUID (Driving Under the Influence of Drugs, Alcohol and Medicines), 2009. Protocol of "Workshop on drug driving detection by means of oral fluid screening", deliverable 
D 3.2.1. p. 11. Retrieved 1st September 2014 from http://www.druidproject.eu/Druid/EN/deliverales-

list/downloads/Deliverable_3_2_1.pdf?_blob=publicationFile.

11. Ley Orgánica 5/2010 de 22 de junio por la que se modifica la Ley Orgánica 10/1995, de 23 de noviembre, del Código Penal. Retrieved 2nd October from http://www.boe.es/boe/dias/2010/06/23/pdfs/BOE-A-2010-9953.pdf

12. Ley 6/2014 de 7 de abril. BOE número 85 de 8/4 /2014. Sec. 1 pp 29508-29524. Retrieved 2nd October 2014 from, http://www.boe.es/boe/dias/2014/04/08/pdfs/BOE-A-2014-3715.pdf. 
